\title{
The Étale Theta Function and Its Frobenioid-Theoretic Manifestations
}

\author{
By
}

Shinichi MochIzUKI*

\begin{abstract}
We develop the theory of the tempered anabelian and Frobenioid-theoretic aspects of the "étale theta function", i.e., the Kummer class of the classical formal algebraic theta function associated to a Tate curve over a nonarchimedean mixed-characteristic local field. In particular, we consider a certain natural "environment" for the study of the étale theta function, which we refer to as a "mono-theta environment" - essentially a Kummer-theoretic version of the classical theta trivialization - and show that this mono-theta environment satisfies certain remarkable rigidity properties involving cyclotomes, discreteness, and constant multiples, all in a fashion that is compatible with the topology of the tempered fundamental group and the extension structure of the associated tempered Frobenioid.
\end{abstract}

\section{Contents}

$\S 0$. Notations and Conventions

$\S 1$. The Tempered Anabelian Rigidity of the Étale Theta Function

$\S 2$. The Theory of Theta Environments

$\S 3 . \quad$ Tempered Frobenioids

$\S 4$. General Bi-Kummer Theory

$\S 5$. The Étale Theta Function via Tempered Frobenioids

\footnotetext{
Communicated by A. Tamagawa. Received May 16, 2007. Revised December 10, 2008 2000 Mathematics Subject Classification(s): Primary 14H42; Secondary 14H30.

* Research Institute for Mathematical Sciences, Kyoto University, Kyoto 606-8502, Japan. e-mail: motizuki@kurims.kyoto-u.ac.jp
} 


\section{Introduction}

The fundamental goal of the present paper is to study the tempered anabelian [cf. [André], [Mzk14]] and Frobenioid-theoretic [cf. [Mzk17], [Mzk18]] aspects of the theta function of a Tate curve over a nonarchimedean mixedcharacteristic local field. The motivation for this approach to the theta function arises from the long-term goal of overcoming various obstacles that occur when one attempts to apply the Hodge-Arakelov theory of elliptic curves [cf. [Mzk4], [Mzk5]; [Mzk6], [Mzk7], [Mzk8], [Mzk9], [Mzk10]] to the diophantine geometry of elliptic curves over number fields. That is to say, the theory of the present paper is motivated by the expectation that these obstacles may be overcome by translating the [essentially] scheme-theoretic formulation of Hodge-Arakelov theory into the language of the geometry of categories [e.g., the "temperoids" of [Mzk14], and the "Frobenioids" of [Mzk17], [Mzk18]]. In certain respects, this situation is reminiscent of the well-known classical solution to the problem of relating the dimension of the first cohomology group of the structure sheaf of a smooth proper variety in positive characteristic to the dimension of its Picard variety - a problem whose solution remained elusive until the foundations of the algebraic geometry of varieties were reformulated in the language of schemes [i.e., one allows for the possibility of nilpotent sections of the structure sheaf].

Since Hodge-Arakelov theory centers around the theory of the theta function of an elliptic curve with bad multiplicative reduction [i.e., a "Tate curve"], it is natural to attempt to begin such a translation by concentrating on such theta functions on Tate curves, as is done in the present paper. Indeed, HodgeArakelov theory may be thought of as a sort of "canonical analytic continuation" of the theory of theta functions on Tate curves to elliptic curves over number fields. Here, we recall that although classically, the arithmetic theory of theta functions on Tate curves is developed in the language of formal schemes in, for instance, [Mumf] [cf. [Mumf], pp. 306-307], this theory only addresses the "slope zero portion" of the theory - i.e., the portion of the theory that involves the quotient of the fundamental group of the generic fiber of the Tate curve that extends to an étale covering in positive characteristic. From this point of view, the relation of the theory of $\S 1, \S 2$ of the present paper to the theory of [Mumf] may be regarded as roughly analogous to the relation of the theory of $p$-adic uniformizations of hyperbolic curves developed in [Mzk1] to Mumford's theory of Schottky uniformations of hyperbolic curves [cf., e.g., [Mzk1], Introduction, $\S 0.1$; cf. also Remark 5.10.2 of the present paper].

Frequently in classical scheme-theoretic constructions, such as those that 
appear in the scheme-theoretic formulation of Hodge-Arakelov theory, there is a tendency to make various arbitrary choices in situations where, a priori, some sort of indeterminacy exists, without providing any sort of intrinsic justification for these choices. Typical examples of such choices involve the choice of a particular rational function or section of a line bundle among various possibilities related by a constant multiple, or the choice of a natural identification between various "cyclotomes" [i.e., isomorphic copies of the module of $N$-th roots of unity, for $N \geq 1$ an integer] appearing in a situation [cf., e.g., [Mzk13], Theorem 4.3, for an example of a crucial rigidity result in anabelian geometry concerning this sort of "choice"].

One important theme of the present paper is the study of phenomena that involve some sort of "extraordinary rigidity" [cf. Grothendieck's famous use of this expression in describing his anabelian philosophy]. This sort of "extraordinary rigidity" may be thought of as a manifestation of the very strong canonicality present in the theory of theta functions on Tate curves that allows one, in Hodge-Arakelov theory, to effect a "canonical analytic continuation" of this theory on Tate curves to elliptic curves over global number fields. The various examples of "extraordinary rigidity" that appear in the theory of the étale theta function may be thought of as examples of intrinsic, category-theoretic "justifications" for the arbitrary choices that appear in classical scheme-theoretic discussions. Such category-theoretic "justifications" depend heavily on the "proper category-theoretic formulation" of various scheme-theoretic "venues". In the theory of the present paper, one central such category-theoretic formulation is a mathematical structure that we shall refer to as a mono-theta environment [cf. Definition 2.13, (ii)]. Roughly speaking:

The mono-theta environment is essentially a Kummer-theoretic version - i.e., a Galois-theoretic version obtained by extracting various $N$-th roots [for $N \geq 1$ an integer] - of the theta trivialization that appears in classical formal scheme-theoretic discussions of the theta function on a Tate curve.

A mono-theta environment may be thought of as a sort of common core for, or bridge between, the [tempered] étale- and Frobenioid-theoretic approaches to the étale theta function [cf. Remarks 2.18.2, 5.10.1, 5.10.2, 5.10.3].

We are now ready to discuss the main results of the present paper. First, we remark that

the reader who is only interested in the definition and basic tempered anabelian properties of the étale theta function may restrict his/her 
attention to the theory of $\S 1$, the main result of which is a tempered anabelian construction of the étale theta function [cf. Theorems 1.6, 1.10].

On the other hand, the main results of the bulk of the paper, which concern more subtle rigidity properties of the étale theta function involving associated mono-theta environments and tempered Frobenioids, may be summarized as follows:

A mono-theta environment is a category/group-theoretic invariant of the tempered étale fundamental group [cf. Corollary 2.18] associated to [certain coverings of] a punctured elliptic curve [satisfying certain properties] over a nonarchimedean mixed-characteristic local field, on the one hand, and of a certain tempered Frobenioid [cf. Theorem 5.10, (iii)] associated to such a curve, on the other. Moreover, a mono-theta environment satisfies the following rigidity properties:

(a) cyclotomic rigidity [cf. Corollary 2.19, (i); Remark 2.19.4];

(b) discrete rigidity [cf. Corollary 2.19, (ii); Remarks 2.16.1, 2.19.4];

(c) constant multiple rigidity [cf. Corollary 2.19, (iii); Remarks 5.12.3, 5.12.5]

- all in a fashion that is compatible with the topology of the tempered fundamental group as well as with the extension structure of the tempered Frobenioid [cf. Corollary 5.12 and the discussion of the following remarks].

In particular, the phenomenon of "cyclotomic rigidity" gives a "categorytheoretic" explanation for the special role played by the first power [i.e., as opposed to the $M$-th power, for $M>1$ an integer] of [the $l$-th root, when one works with $l$-torsion points, for $l \geq 1$ an odd integer, of] the theta function [cf. Remarks 2.19.2, 2.19.3, 5.10.3, 5.12.5] - a phenomenon which may also be seen in "scheme-theoretic" Hodge-Arakelov theory [a theory that also effectively involves [sections of] the first power of some ample line bundle, and which does not generalize in any evident way to arbitrary powers of this ample line bundle].

One further interesting aspect of cyclotomic rigidity in the theory of the present paper is that it is obtained essentially as the result of a certain computation involving commutators [cf. Remark 2.19.2]. Put another way, one may think of this cyclotomic rigidity as a sort of consequence of the nonabelian 
structure of [what essentially amounts, from a more classical point of view, to] the "theta-group". This application of the structure of the theta-group differs substantially from the way in which the structure of the theta-group is used in more classical treatments of the theory of theta functions - namely, to show that certain representations, such as those arising from theta functions, are irreducible. One way to understand this difference is as follows. Whereas classical treatments that center around such irreducibility results only treat certain $l$-dimensional subspaces of the $l^{2}$-dimensional space of set-theoretic functions on the $l$-torsion points of an elliptic curve, Hodge-Arakelov theory is concerned with understanding ["modulo" the $l$-dimensional subspace which has already been well understood in the classical theory!] the entire $l^{2}$-dimensional function space that appears [cf. [Mzk4], §1.1]. Put another way, the $l$-dimensional subspace which forms the principal topic of the classical theory may be thought of as corresponding to the space of holomorphic functions [cf. [Mzk4], §1.4.2]; by contrast, Hodge-Arakelov theory — cf., e.g., the arithmetic Kodaira-Spencer morphism of [Mzk4], §1.4 - is concerned with understanding deformations of the holomorphic structure, from an arithmetic point of view. Moreover, from the point of view of considering such deformations of holomorphic structure, it is convenient, and, indeed, more efficient, to work "modulo variations contained within the subspace corresponding to the holomorphic functions" - which, at any rate, may be treated, as a consequence of the classical theory of irreducible representations of the theta-group, as a single "irreducible unit"! This is precisely the point of view of the "Lagrangian approach" of [Mzk5], §3, an approach which allows one to work "modulo the $l$-dimensional subspace of the classical theory", by applying various isogenies that allow one to replace the "entire $l^{2}$-dimensional function space" associated to the original elliptic curve by an l-dimensional function space that is suited to studying "arithmetic deformations of holomorphic structure" in the style of Hodge-Arakelov theory. In the present paper, these isogenies of the "Lagrangian approach to Hodge-Arakelov theory" correspond to the various coverings that appear in the discussion at the beginning of $\S 2$; the resulting "l-dimensional function space" then corresponds, in the theory of the present paper, to the space of functions on the labels that appear in Corollary 2.9.

Here, it is important to note that although the above three rigidity properties may be stated and understood to a certain extent without reference to the Frobenioid-theoretic portion of the theory [cf. §2], certain aspects of the interdependence of these rigidity properties, as well as the meaning of establishing these rigidity properties under the condition of compatibility with the topology 
of the tempered fundamental group as well as with the extension structure of the tempered Frobenioid, may only be understood in the context of the theory of Frobenioids [cf. Corollary 5.12 and the discussion of the following remarks]. Indeed, one important theme of the theory of the present paper - which may, roughly, be summarized as the idea that sometimes

"less (respectively, more) data yields more (respectively, less) information"

[cf. Remark 5.12.9] — is precisely the study of the rather intricate way in which these various rigidity/compatibility properties are related to one another. Typically speaking, the reason for this [at first glance somewhat paradoxical] phenomenon is that "more data" means more complicated systems made up of various components, hence obligates one to keep track of the various indeterminacies that arise in relating [i.e., without resorting to the use of "arbitrary choices"] these components to one another. If these indeterminacies are sufficiently severe, then they may have the effect of obliterating certain structures that one is interested in. By contrast, if certain portions of such a system are redundant, i.e., in fact uniquely and rigidly - i.e., "canonically" - determined by more fundamental portions of the system, then one need not contend with the indeterminacies that arise from relating the redundant components; this yields a greater chance that the structures of interest are not obliterated by the intrinsic indeterminacies of the system.

One way to appreciate the "tension" that exists between the various rigidity properties satisfied by the mono-theta environment is by comparing the theory of the mono-theta environment to that of the bi-theta environment [cf. Definition 2.13, (iii)]. The bi-theta environment is essentially a Kummer-theoretic version of the pair of sections - corresponding to the "numerator" and "denominator" of the theta function - of a certain ample line bundle on a Tate curve. One of these two sections is the theta trivialization that appears in the mono-theta environment; the other of these two sections is the "algebraic section" that arises tautologically from the original definition of the ample line bundle.

The bi-theta environment satisfies cyclotomic rigidity and constant multiple rigidity properties for somewhat more evident reasons than the mono-theta environment. In the case of constant multiple rigidity, this arises partly from the fact that the bi-theta environment involves working, in essence, with a ratio [i.e., in the form of a "pair"] of sections, hence is immune to the operation of multiplying both sections by a constant [cf. Remarks $5.10 .4 ; 5.12 .7$, (ii)]. On the other hand, the bi-theta environment fails to satisfy the discrete rigidity 
property satisfied by the mono-theta environment [cf. Corollary 2.16; Remark 2.16.1]. By contrast, the mono-theta environment satisfies all three rigidity properties, despite the fact that cyclotomic rigidity [cf. the proof of Corollary 2.19, (i); Remark 2.19.2] and constant multiple rigidity [cf. Remarks 5.12.3, 5.12.5] are somewhat more subtle.

Here, it is interesting to note that the issue of discrete rigidity for the mono-theta and bi-theta environments revolves, in essence, around the fact that $\widehat{\mathbb{Z}} / \mathbb{Z} \neq 0$, i.e., the gap between $\mathbb{Z}$ and its profinite completion $\widehat{\mathbb{Z}}$ - cf. Remark 2.16.1. On the other hand, the subtlety of constant multiple rigidity for mono-theta environments revolves, in essence, around the nontriviality of the extension structure of a Frobenoid [i.e., as an "extension by line bundles of the base category" - cf. Remarks 5.10.2, 5.12.3, 5.12.5, 5.12.7]. Put another way:

The mono-theta environment may be thought of as a sort of translation apparatus that serves to translate the "global arithmetic gap between $\mathbb{Z}$ and $\widehat{\mathbb{Z}}$ " [cf. Remark 2.16.2 for more on the relation of this portion of the theory to global arithmetic bases] into the "nontriviality of the local geometric extension" constituted by the extension structure of the tempered Frobenioid under consideration.

This sort of relationship between the global arithmetic gap between $\mathbb{Z}$ and $\widehat{\mathbb{Z}}$ and the theory of theta functions is reminiscent of the point of view that theta functions are related to "splittings of the natural surjection $\mathbb{Z} \rightarrow \mathbb{Z} / N \mathbb{Z}$ ", a point of view that arises in Hodge-Arakelov theory [cf. [Mzk4], §1.3.3].

The contents of the present paper are organized as follows. In $\S 1$, we discuss the purely tempered étale-theoretic anabelian aspects of the theta function and show, in particular, that the "étale theta function" - i.e., the Kummer class of the usual formal algebraic theta function - is preserved by isomorphisms of the tempered fundamental group [cf. Theorems 1.6; 1.10]. In $\S 2$, after studying various coverings and quotient coverings of a punctured elliptic curve, we introduce the notions of a mono-theta environment and a bi-theta environment [cf. Definition 2.13] and study the "group-theoretic constructibility" and rigidity properties of these notions [cf. Corollaries 2.18, 2.19]. In §3, we define the "tempered Frobenioids" in which we shall develop the Frobenioidtheoretic approach to the étale theta function; in particular, we show that these tempered Frobenioids satisfy various nice properties which allow one to apply the extensive theory of [Mzk17], [Mzk18] [cf. Theorem 3.7; Corollary 3.8]. In $\S 4$, we develop "bi-Kummer theory" - i.e., a sort of generalization of the "Kummer class associated to a rational function" to the "bi-Kummer 
data" associated to a pair of sections [corresponding to the numerator and denominator of a rational function] of a line bundle - in a category-theoretic fashion [cf. Theorem 4.4] for fairly general tempered Frobenioids. Finally, in $\S 5$, we specialize the theory of $\S 3, \S 4$ to the case of the étale theta function, as discussed in $\S 1$. In particular, we observe that a mono-theta environment may also be regarded as a mathematical structure naturally associated to a certain tempered Frobenioid [cf. Theorem 5.10, (iii)]. Also, we discuss certain aspects of the constant multiple rigidity [as well as, to a lesser extent, of the cyclotomic and discrete rigidity] of a mono-theta environment that may only be understood in the context of the Frobenioid-theoretic approach to the étale theta function [cf. Corollary 5.12 and the discussion of the following remarks].

\section{$\S 0 . \quad$ Notations and Conventions}

In addition to the "Notations and Conventions" of [Mzk17], §0, we shall employ the following "Notations and Conventions" in the present paper:

\section{Monoids:}

We shall denote by $\mathbb{N}_{\geq 1}$ the multiplicative monoid of [rational] integers $\geq 1$ [cf. [Mzk17], §0].

Let $Q$ be a commutative monoid [with unity]; $P \subseteq Q$ a submonoid. If $Q$ is integral [so $Q$ embeds into its groupification $Q^{\mathrm{gp}}$; we have a natural inclusion $\left.P^{\mathrm{gp}} \hookrightarrow Q^{\mathrm{gp}}\right]$, then we shall refer to the submonoid

$$
P^{\mathrm{gp}} \bigcap Q\left(\subseteq Q^{\mathrm{gp}}\right)
$$

of $Q$ as the group-saturation of $P$ in $Q$; if $P$ is equal to its group-saturation in $Q$, then we shall say that $P$ is group-saturated in $Q$. If $Q$ is torsion-free [so $Q$ embeds into its perfection $Q^{\mathrm{pf}}$; we have a natural inclusion $\left.P^{\mathrm{pf}} \hookrightarrow Q^{\mathrm{pf}}\right]$, then we shall refer to the submonoid

$$
P^{\mathrm{pf}} \bigcap Q\left(\subseteq Q^{\mathrm{pf}}\right)
$$

of $Q$ as the perf-saturation of $P$ in $Q$; if $P$ is equal to its perf-saturation in $Q$, then we shall say that $P$ is perf-saturated in $Q$.

\section{Topological Groups:}

Let $\Pi$ be a topological group. Then let us write

$$
\mathcal{B}^{\text {temp }}(\Pi)
$$


for the category whose objects are countable [i.e., of cardinality $\leq$ the cardinality of the set of natural numbers], discrete sets equipped with a continuous $\Pi$-action and whose morphisms are morphisms of П-sets [cf. [Mzk14], §3]. If П may be written as an inverse limit of an inverse system of surjections of countable discrete topological groups, then we shall say that $\Pi$ is tempered [cf. [Mzk14], Definition 3.1, (i)].

We shall refer to a normal open subgroup $H \subseteq \Pi$ such that the quotient group $\Pi / H$ is free as co-free. We shall refer to a co-free subgroup $H \subseteq \Pi$ as minimal if every co-free subgroup of $\Pi$ contains $H$. Thus, a minimal co-free subgroup of $\Pi$ is necessarily unique and characteristic.

\section{Categories:}

We shall refer to an isomorphic copy of some object as an isomorph of the object.

Let $\mathcal{C}$ be a category; $A \in \operatorname{Ob}(\mathcal{C})$. Then we shall write

$$
\mathcal{C}_{A}
$$

for the category whose objects are morphisms $B \rightarrow A$ of $\mathcal{C}$ and whose morphisms [from an object $B_{1} \rightarrow A$ to an object $B_{2} \rightarrow A$ ] are $A$-morphisms $B_{1} \rightarrow B_{2}$ in $\mathcal{C}$ [cf. [Mzk17], §0] and

$$
\mathcal{C}[A] \subseteq \mathcal{C}
$$

for the full subcategory of $\mathcal{C}$ determined by the objects of $\mathcal{C}$ that admit a morphism to $A$. Given two arrows $f_{i}: A_{i} \rightarrow B_{i}$ (where $i=1,2$ ) in $\mathcal{C}$, we shall refer to a commutative diagram

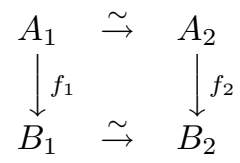

- where the horizontal arrows are isomorphisms in $\mathcal{C}$ - as an abstract equivalence from $f_{1}$ to $f_{2}$. If there exists an abstract equivalence from $f_{1}$ to $f_{2}$, then we shall say that $f_{1}, f_{2}$ are abstractly equivalent.

Let $\Phi: \mathcal{C} \rightarrow \mathcal{D}$ be a faithful functor between categories $\mathcal{C}, \mathcal{D}$. Then we shall say that $\Phi$ is isomorphism-full if every isomorphism $\Phi(A) \stackrel{\sim}{\rightarrow} \Phi(B)$ of $\mathcal{D}$, where $A, B \in \mathrm{Ob}(\mathcal{C})$, arises by applying $\Phi$ to an isomorphism $A \stackrel{\sim}{\rightarrow} B$ of $\mathcal{C}$. Suppose that $\Phi$ is isomorphism-full. Then observe that the objects of $\mathcal{D}$ that are isomorphic to objects in the image of $\Phi$, together with the morphisms of $\mathcal{D}$ that are abstractly equivalent to morphisms in the image of $\Phi$, form a subcategory $\mathcal{C}^{\prime} \subseteq \mathcal{D}$ such that $\Phi$ induces an equivalence of categories $\mathcal{C} \stackrel{\sim}{\rightarrow} \mathcal{C}^{\prime}$. 
We shall refer to this subcategory $\mathcal{C}^{\prime} \subseteq \mathcal{D}$ as the essential image of $\Phi$. [Thus, this terminology is consistent with the usual terminology of "essential image" in the case where $\Phi$ is fully faithful.]

\section{Curves:}

We refer to [Mzk14], §0, for generalities concerning [families of] hyperbolic curves, smooth log curves, stable log curves, divisors of cusps, and divisors of marked points.

If $C^{\log } \rightarrow S^{\log }$ is a stable log curve, and, moreover, $S$ is the spectrum of a field, then we shall say that $C^{\log }$ is split if each of the irreducible components and nodes of $C$ is geometrically irreducible over $S$.

A morphism of log stacks

$$
C^{\log } \rightarrow S^{\log }
$$

for which there exists an étale surjection $S_{1} \rightarrow S$, where $S_{1}$ is a scheme, such that $C_{1}^{\log \stackrel{\text { def }}{=}} C^{\log } \times_{S} S_{1}$ may be obtained as the result of forming the quotient [in the sense of log stacks!] of a stable (respectively, smooth) $\log$ curve $C_{2}^{\log } \rightarrow$ $S_{1}^{\log \stackrel{\text { def }}{=}} S^{\log } \times_{S} S_{1}$ by the action of a finite group of automorphisms of $C_{2}^{\log }$ over $S_{1}^{\log }$ which acts freely on a dense open subset of every fiber of $C_{2} \rightarrow S_{1}$ will be referred to as a stable log orbicurve (respectively, smooth log orbicurve) over $S^{\log }$. Thus, the divisor of cusps of $C_{2}^{\text {log }}$ determines a divisor of cusps of $C_{1}^{\log }, C^{\log }$. Here, if $C_{2}^{\log } \rightarrow S_{1}^{\log }$ is of type $(1,1)$, and the finite group of automorphisms is given by the action of " \pm 1 " [i.e., relative to the group structure of the underlying elliptic curve of $\left.C_{2}^{\log } \rightarrow S_{1}^{\log }\right]$, then the resulting stable log orbicurve will be referred to as being of type $(1,1)_{ \pm}$.

If $S^{\log }$ is the spectrum of a field, equipped with the trivial log structure, then a hyperbolic orbicurve $X \rightarrow S$ is defined to be the algebraic [log] stack [with trivial $\log$ structure] obtained by removing the divisor of cusps from some smooth log orbicurve $C^{\log } \rightarrow S^{\log }$ over $S^{\log }$. If $X$ (respectively, $Y$ ) is a hyperbolic orbicurve over a field $K$ (respectively, $L$ ), then we shall say that $X$ is isogenous to $Y$ if there exists a hyperbolic curve $Z$ over a field $M$ together with finite étale morphisms $Z \rightarrow X, Z \rightarrow Y$. Note that in this situation, the morphisms $Z \rightarrow X, Z \rightarrow Y$ induce finite separable inclusions of fields $K \hookrightarrow M, L \hookrightarrow M$. [Indeed, this follows immediately from the easily verified fact that every subgroup $G \subseteq \Gamma\left(Z, \mathcal{O}_{Z}^{\times}\right)$such that $G \bigcup\{0\}$ determines a field is necessarily contained in $M^{\times}$.] 


\section{$\S 1$. The Tempered Anabelian Rigidity of the Étale Theta Function}

In this $\S$, we construct a certain cohomology class in the [continuous] group cohomology of the tempered fundamental group of a once-punctured elliptic curve which may be regarded as a sort of "tempered analytic" representation of the theta function. We then discuss various properties of this "étale theta function". In particular, we apply the theory of [Mzk14], 66 , to show that it is, up to certain relatively mild indeterminacies, preserved by arbitrary automorphisms of the tempered fundamental group [cf. Theorems 1.6, 1.10].

Let $K$ be a finite extension of $\mathbb{Q}_{p}$, with ring of integers $\mathcal{O}_{K} ; \bar{K}$ an algebraic closure of $K ; \mathfrak{S}$ the formal scheme determined by the $p$-adic completion of $\operatorname{Spec}\left(\mathcal{O}_{K}\right) ; \mathfrak{S}^{\log }$ the formal $\log$ scheme obtained by equipping $\mathfrak{S}$ with the $\log$ structure determined by the unique closed point of $\operatorname{Spec}\left(\mathcal{O}_{K}\right) ; \mathfrak{X}^{\log }$ a stable log curve over $\mathfrak{S}^{\log }$ of type $(1,1)$. Also, we assume that the special fiber of $\mathfrak{X}$ is singular and split [cf. §0], and that the generic fiber of the algebrization of $\mathfrak{X}^{\log }$

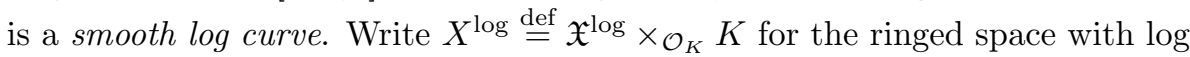
structure obtained by tensoring the structure sheaf of $\mathfrak{X}$ over $\mathcal{O}_{K}$ with $K$. In the following discussion, we shall often [by abuse of notation] use the notation $X^{\log }$ also to denote the generic fiber of the algebrization of $\mathfrak{X}^{\log }$. [Here, the reader should note that these notational conventions differ somewhat from notational conventions typically employed in discussions of rigid-analytic geometry.]

Let us write

$$
\underline{\Pi}_{X}^{\mathrm{tp}}
$$

for the tempered fundamental group associated to $X^{\log }$ [cf. [André], §4; the group " $\pi_{1}^{\text {temp }}\left(X_{K}^{\log }\right)$ " of [Mzk14], Examples 3.10, 5.6], with respect to some basepoint. The issue of how our constructions are affected when the basepoint varies will be studied in the present paper by considering to what extent these constructions are preserved by inner or, more generally, arbitrary isomorphisms between fundamental groups [cf. Remark 1.6.2 below]. Here, despite the fact that the [tempered] fundamental group in question is best thought of not as "the fundamental group of $X$ " but rather as "the fundamental group of $X^{\log }$ ", we use the notation " $\underline{\Pi}_{X}^{\mathrm{tp}}$ " rather than " $\Pi_{X \log }^{\mathrm{tp}}$ " in order to minimize the number of subscripts and superscripts that appear in the notation [cf. the discussion to follow in the remainder of the present paper!]; thus, the reader should think of the underlined notation " $\underline{\Pi}_{(-)}^{\mathrm{tp}}$ " as an abbreviation for the "logarithmic tempered fundamental group of the scheme (-), equipped with the log structure currently under consideration", i.e., an abbreviation for " $\pi_{1}^{\text {temp }}\left((-)^{\log }\right)^{\text {". }}$

Denote by $\underline{\Delta}_{X}^{\mathrm{tp}} \subseteq \underline{\Pi}_{X}^{\mathrm{tp}}$ the "geometric tempered fundamental group". Thus, 
we have a natural exact sequence

$$
1 \rightarrow \underline{\Delta}_{X}^{\mathrm{tp}} \rightarrow \underline{\Pi}_{X}^{\mathrm{tp}} \rightarrow G_{K} \rightarrow 1
$$

— where $G_{K} \stackrel{\text { def }}{=} \operatorname{Gal}(\bar{K} / K)$.

Since the special fiber of $\mathfrak{X}$ is split, it follows that the universal graphcovering of the dual graph of this special fiber determines [up to composition with an element of $\operatorname{Aut}(\mathbb{Z})=\{ \pm 1\}]$ a natural surjection

$$
\underline{\Pi}_{X}^{\mathrm{tp}} \rightarrow \mathbb{Z}
$$

whose kernel, which we denote by $\underline{\Pi}_{Y}^{\mathrm{tp}}$, determines an infinite étale covering

$$
\mathfrak{Y}^{\log } \rightarrow \mathfrak{X}^{\log }
$$

- i.e., $\mathfrak{Y}^{\log }$ is a $p$-adic formal scheme equipped with a log structure; the special fiber of $\mathfrak{Y}$ is an infinite chain of copies of the projective line, joined at 0 and

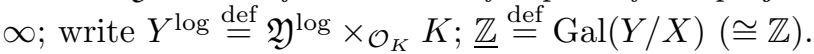

Write $\underline{\Pi}_{X} \stackrel{\text { def }}{=}\left(\underline{\Pi}_{X}^{\text {tp }}\right)^{\wedge} ; \underline{\Delta}_{X} \stackrel{\text { def }}{=}\left(\underline{\Delta}_{X}^{\text {tp }}\right)^{\wedge}$ [where the "^^" denotes the profinite completion]. Then we have a natural exact sequence

$$
1 \rightarrow \widehat{\mathbb{Z}}(1) \rightarrow \underline{\Delta}_{X}^{\text {ell }} \rightarrow \widehat{\mathbb{Z}} \rightarrow 1
$$

— where we write $\underline{\Delta}_{X}^{\text {ell }} \stackrel{\text { def }}{=} \underline{\Delta}_{X}^{\mathrm{ab}}=\underline{\Delta}_{X} /\left[\underline{\Delta}_{X}, \underline{\Delta}_{X}\right]$ for the abelianization of $\underline{\Delta}_{X}$. Since $\Delta_{X}$ is a profinite free group on 2 generators, we also have a natural exact sequence

$$
1 \rightarrow \wedge^{2} \underline{\Delta}_{X}^{\mathrm{ell}}(\cong \widehat{\mathbb{Z}}(1)) \rightarrow \underline{\Delta}_{X}^{\Theta} \rightarrow \underline{\Delta}_{X}^{\mathrm{ell}} \rightarrow 1
$$

— where we write $\underline{\Delta}_{X}^{\ominus} \stackrel{\text { def }}{=} \underline{\Delta}_{X} /\left[\underline{\Delta}_{X},\left[\underline{\Delta}_{X}, \underline{\Delta}_{X}\right]\right]$. Let us denote the image of $\wedge^{2} \underline{\Delta}_{X}^{\text {ell }}$ in $\underline{\Delta}_{X}^{\Theta}$ by $(\widehat{\mathbb{Z}}(1) \cong) \underline{\Delta}_{\Theta} \subseteq \underline{\Delta}_{X}^{\Theta}$. Similarly, we have natural exact sequences

$$
\begin{gathered}
1 \rightarrow \widehat{\mathbb{Z}}(1) \rightarrow\left(\underline{\Delta}_{X}^{\mathrm{tp}}\right)^{\mathrm{ell}} \rightarrow \underline{\mathbb{Z}} \rightarrow 1 \\
1 \rightarrow \underline{\Delta}_{\Theta} \rightarrow\left(\underline{\Delta}_{X}^{\mathrm{tp}}\right)^{\Theta} \rightarrow\left(\underline{\Delta}_{X}^{\mathrm{tp}}\right)^{\mathrm{ell}} \rightarrow 1
\end{gathered}
$$

— where we write

$$
\underline{\Delta}_{X}^{\mathrm{tp}} \rightarrow\left(\underline{\Delta}_{X}^{\mathrm{tp}}\right)^{\Theta} \rightarrow\left(\underline{\Delta}_{X}^{\mathrm{tp}}\right)^{\mathrm{ell}}
$$

for the quotients induced by the quotients $\underline{\Delta}_{X} \rightarrow \underline{\Delta}_{X}^{\ominus} \rightarrow \underline{\Delta}_{X}^{\text {ell }}$. Also, we shall write

$$
\underline{\Pi}_{X}^{\mathrm{tp}} \rightarrow\left(\underline{\Pi}_{X}^{\mathrm{tp}}\right)^{\Theta} \rightarrow\left(\underline{\Pi}_{X}^{\mathrm{tp}}\right)^{\mathrm{ell}}
$$

for the quotients whose kernels are the kernels of the quotients $\underline{\Delta}_{X}^{\mathrm{tp}} \rightarrow\left(\underline{\Delta}_{X}^{\mathrm{tp}}\right)^{\Theta} \rightarrow$ $\left(\underline{\Delta}_{X}^{\text {tp }}\right)^{\text {ell }}$ and

$$
\underline{\Pi}_{Y}^{\mathrm{tp}} \rightarrow\left(\underline{\Pi}_{Y}^{\mathrm{tp}}\right)^{\Theta} \rightarrow\left(\underline{\Pi}_{Y}^{\mathrm{tp}}\right)^{\mathrm{ell}} ; \quad \underline{\Delta}_{Y}^{\mathrm{tp}} \rightarrow\left(\underline{\Delta}_{Y}^{\mathrm{tp}}\right)^{\Theta} \rightarrow\left(\underline{\Delta}_{Y}^{\mathrm{tp}}\right)^{\mathrm{ell}}
$$


for the quotients of $\underline{\Pi}_{Y}^{\mathrm{tp}}, \underline{\Delta}_{Y}^{\mathrm{tp}}$ induced by the quotients of $\underline{\Pi}_{X}^{\mathrm{tp}}, \underline{\Delta}_{X}^{\mathrm{tp}}$ with similar superscripts. Thus, $\left(\underline{\Delta}_{Y}^{\text {tp }}\right)^{\text {ell }} \cong \widehat{\mathbb{Z}}(1)$; we have a natural exact sequence of abelian profinite groups $1 \rightarrow \underline{\Delta}_{\Theta} \rightarrow\left(\underline{\Delta}_{Y}^{\mathrm{tp}}\right)^{\Theta} \rightarrow\left(\underline{\Delta}_{Y}^{\mathrm{tp}}\right)^{\text {ell }} \rightarrow 1$.

Next, let us write $q_{X} \in \mathcal{O}_{K}$ for the q-parameter of the underlying elliptic curve of $X^{\log }$. If $N \geq 1$ is an integer, set

$$
K_{N} \stackrel{\text { def }}{=} K\left(\zeta_{N}, q_{X}^{1 / N}\right) \subseteq \bar{K}
$$

- where $\zeta_{N}$ is a primitive $N$-th root of unity. Then any decomposition group of a cusp of $Y^{\text {log }}$ determines, up to conjugation by $\left(\underline{\Delta}_{Y}^{\text {tp }}\right)^{\text {ell }}$, a section $G_{K} \rightarrow$ $\left(\underline{\Pi}_{Y}^{\mathrm{tp}}\right)^{\text {ell }}$ of the natural surjection $\left(\underline{\Pi}_{Y}^{\mathrm{tp}}\right)^{\text {ell }} \rightarrow G_{K}$ whose restriction to the open subgroup $G_{K_{N}} \subseteq G_{K}$ determines an open immersion

$$
G_{K_{N}} \hookrightarrow\left(\underline{\Pi}_{Y}^{\mathrm{tp}}\right)^{\mathrm{ell}} / N \cdot\left(\underline{\Delta}_{Y}^{\mathrm{tp}}\right)^{\mathrm{ell}}
$$

the image of which is stabilized by the conjugation action of $\underline{\Pi}_{X}^{\mathrm{tp}}$. [Indeed, this follows from the fact that $G_{K_{N}}$ acts trivially on $\left(\underline{\Delta}_{X}^{\text {tp }}\right)^{\text {ell }} / N \cdot\left(\underline{\Delta}_{Y}^{\text {tp }}\right)^{\text {ell }}$.] Thus, this image determines a Galois covering

$$
Y_{N} \rightarrow Y
$$

such that the resulting surjection $\underline{\Pi}_{Y}^{\mathrm{tp}} \rightarrow \operatorname{Gal}\left(Y_{N} / Y\right)$, whose kernel we denote by $\underline{\Pi}_{Y_{N}}^{\mathrm{tp}}$, induces a natural exact sequence $1 \rightarrow\left(\underline{\Delta}_{Y}^{\mathrm{tp}}\right)^{\mathrm{ell}} \otimes \mathbb{Z} / N \mathbb{Z} \rightarrow \operatorname{Gal}\left(Y_{N} / Y\right) \rightarrow$ $\operatorname{Gal}\left(K_{N} / K\right) \rightarrow 1$. Also, we shall write

$$
\underline{\Pi}_{Y_{N}}^{\mathrm{tp}} \rightarrow\left(\underline{\Pi}_{Y_{N}}^{\mathrm{tp}}\right)^{\Theta} \rightarrow\left(\underline{\Pi}_{Y_{N}}^{\mathrm{tp}}\right)^{\mathrm{ell}} ; \quad \underline{\Delta}_{Y_{N}}^{\mathrm{tp}} \rightarrow\left(\underline{\Delta}_{Y_{N}}^{\mathrm{tp}}\right)^{\Theta} \rightarrow\left(\underline{\Delta}_{Y_{N}}^{\mathrm{tp}}\right)^{\mathrm{ell}}
$$

for the quotients of $\underline{\Pi}_{Y_{N}}^{\mathrm{tp}}, \underline{\Delta}_{Y_{N}}^{\mathrm{tp}}$ induced by the quotients of $\underline{\Pi}_{Y}^{\mathrm{tp}}, \underline{\Delta}_{Y}^{\mathrm{tp}}$ with similar superscripts and

$$
Y_{N}^{\log }
$$

for the object obtained by equipping $Y_{N}$ with the log structure determined by the $K_{N}$-valued points of $Y_{N}$ lying over the cusps of $Y$. Set

$$
\mathfrak{Y}_{N} \rightarrow \mathfrak{Y}
$$

equal to the normalization of $\mathfrak{Y}$ in $Y_{N}$. One verifies easily that the special fiber of $\mathfrak{Y}_{N}$ is an infinite chain of copies of the projective line, joined at 0 and $\infty$; each of these points " 0 " and " $\infty$ " is a node on $\mathfrak{Y}_{N}$; each projective line in this chain maps to a projective line in the special fiber of $\mathfrak{Y}$ by the " $N$-th power map" on the copy of " $\mathbb{G}_{m}$ " obtained by removing the nodes; if we choose some irreducible component of the special fiber of $\mathfrak{Y}$ as a "basepoint", then the 
natural action of $\underline{\mathbb{Z}}$ on $\mathfrak{Y}$ allows one to think of the projective lines in the special fiber of $\mathfrak{Y}$ as being labeled by elements of $\underline{\mathbb{Z}}$. In particular, it follows immediately that the isomorphism class of a line bundle on $\mathfrak{Y}_{N}$ is completely determined by the degree of the restriction of the line bundle to each of these copies of the projective line. That is to say, these degrees determine an isomorphism

$$
\operatorname{Pic}\left(\mathfrak{Y}_{N}\right) \stackrel{\sim}{\rightarrow} \mathbb{Z}^{\mathbb{Z}}
$$

— where $\mathbb{Z} \underline{\mathbb{Z}}$ denotes the module of functions $\underline{\mathbb{Z}} \rightarrow \mathbb{Z}$; the additive structure on this module is induced by the additive structure on the codomain " $\mathbb{Z}$ ". Write

$$
\mathfrak{L}_{N}
$$

for the line bundle on $\mathfrak{Y}_{N}$ determined by the constant function $\underline{\mathbb{Z}} \rightarrow \mathbb{Z}$ whose value is 1 . Also, we observe that it follows immediately from the above explicit description of the special fiber of $\mathfrak{Y}_{N}$ that $\Gamma\left(\mathfrak{Y}_{N}, \mathcal{O}_{\mathfrak{Y}_{N}}\right)=\mathcal{O}_{K_{N}}$.

Next, write

$$
J_{N} \stackrel{\text { def }}{=} K_{N}\left(a^{1 / N}\right)_{a \in K_{N}} \subseteq \bar{K}
$$

— where we note that [since $K_{N}^{\times}$is topologically finitely generated] $J_{N}$ is a finite Galois extension of $K_{N}$. Observe, moreover, that we have an exact sequence

$$
1 \rightarrow \underline{\Delta}_{\Theta} \otimes \mathbb{Z} / N \mathbb{Z}(\cong \mathbb{Z} / N \mathbb{Z}(1)) \rightarrow\left(\underline{\Pi}_{Y_{N}}^{\mathrm{tp}}\right)^{\Theta} / N \cdot\left(\underline{\Delta}_{Y}^{\mathrm{tp}}\right)^{\Theta} \rightarrow G_{K_{N}} \rightarrow 1
$$

[cf. the construction of $Y_{N}$ ]. Since any two splittings of this exact sequence differ by a cohomology class $\in H^{1}\left(G_{K_{N}}, \mathbb{Z} / N \mathbb{Z}(1)\right)$, it follows [by the definition of $J_{N}$ ] that all splittings of this exact sequence determine the same splitting over $G_{J_{N}}$. Thus, the image of the resulting open immersion

$$
G_{J_{N}} \hookrightarrow\left(\underline{\Pi}_{Y_{N}}^{\mathrm{tp}}\right)^{\Theta} / N \cdot\left(\underline{\Delta}_{Y}^{\mathrm{tp}}\right)^{\Theta}
$$

is stabilized by the conjugation action of $\underline{\Pi}_{X}^{\mathrm{tp}}$, hence determines a Galois covering

$$
Z_{N} \rightarrow Y_{N}
$$

such that the resulting surjection $\underline{\Pi}_{Y_{N}}^{\mathrm{tp}} \rightarrow \operatorname{Gal}\left(Z_{N} / Y_{N}\right)$, whose kernel we denote by $\underline{\Pi}_{Z_{N}}^{\text {tp }}$, induces a natural exact sequence $1 \rightarrow \underline{\Delta}_{\Theta} \otimes \mathbb{Z} / N \mathbb{Z} \rightarrow \operatorname{Gal}\left(Z_{N} / Y_{N}\right) \rightarrow$ $\operatorname{Gal}\left(J_{N} / K_{N}\right) \rightarrow 1$. Also, we shall write

$$
\underline{\Pi}_{Z_{N}}^{\text {tp }} \rightarrow\left(\underline{\Pi}_{Z_{N}}^{\text {tp }}\right)^{\Theta} \rightarrow\left(\underline{\Pi}_{Z_{N}}^{\text {tp }}\right)^{\text {ell }} ; \quad \underline{\Delta}_{Z_{N}}^{\text {tp }} \rightarrow\left(\underline{\Delta}_{Z_{N}}^{\text {tp }}\right)^{\Theta} \rightarrow\left(\underline{\Delta}_{Z_{N}}^{\text {tp }}\right)^{\text {ell }}
$$

for the quotients of $\underline{\Pi}_{Z_{N}}^{\mathrm{tp}}, \underline{\Delta}_{Z_{N}}^{\mathrm{tp}}$ induced by the quotients of $\underline{\Pi}_{Y_{N}}^{\mathrm{tp}}$, ${\underline{Y_{N}}}_{\mathrm{t}}^{\mathrm{tp}}$ with similar superscripts and

$$
Z_{N}^{\log }
$$


for the object obtained by equipping $Z_{N}$ with the log structure determined by the [manifestly] $J_{N}$-valued points of $Z_{N}$ lying over the cusps of $Y$. Set

$$
\mathfrak{Z}_{N} \rightarrow \mathfrak{Y}_{N}
$$

equal to the normalization of $\mathfrak{Y}$ in $Z_{N}$. Since $\mathfrak{Y}$ is "generically of characteristic zero" [i.e., $Y$ is of characteristic zero], it follows that $\mathfrak{Z}_{N}$ is finite over $\mathfrak{Y}$.

Next, let us observe that there exists a section

$$
s_{1} \in \Gamma\left(\mathfrak{Y}=\mathfrak{Y}_{1}, \mathfrak{L}_{1}\right)
$$

- well-defined up to an $\mathcal{O}_{K}^{\times}$-multiple - whose zero locus on $\mathfrak{Y}$ is precisely the divisor of cusps of $\mathfrak{Y}$. Also, let us fix an isomorphism of $\mathfrak{L}_{N}^{\otimes N}$ with $\left.\mathfrak{L}_{1}\right|_{\mathfrak{Y}_{N}}$, which we use to identify these two bundles. Note that there is a natural action of $\operatorname{Gal}(Y / X)$ on $\mathfrak{L}_{1}$ which is uniquely determined by the condition that it preserve $s_{1}$. Thus, we obtain a natural action of $\operatorname{Gal}\left(Y_{N} / X\right)$ on $\left.\mathfrak{L}_{1}\right|_{\mathfrak{Y}_{N}}$.

Proposition 1.1 (Theta Action of the Tempered Fundamental Group).

(i) The section

$$
\left.s_{1}\right|_{\mathfrak{Y}_{N}} \in \Gamma\left(\mathfrak{Y}_{N},\left.\mathfrak{L}_{1}\right|_{\mathfrak{Y}} \cong \mathfrak{L}_{N}^{\otimes N}\right)
$$

admits an $N$-th root $s_{N} \in \Gamma\left(\mathfrak{Z}_{N},\left.\mathfrak{L}_{N}\right|_{\mathfrak{Z}_{N}}\right)$ over $\mathfrak{Z}_{N}$. In particular, if we denote associated "geometric line bundles" by the notation " $\mathbb{V}(-)$ ", then we obtain a commutative diagram

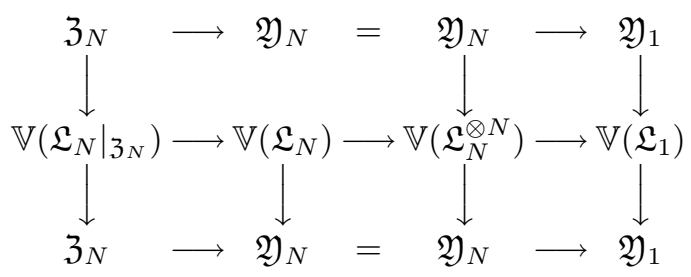

- where the horizontal morphisms in the first and last lines are the natural morphisms; in the second line of horizontal morphisms, the first and third horizontal morphisms are the pull-back morphisms, while the second morphism is given by raising to the $\mathrm{N}$-th power; in the first row of vertical morphisms, the morphism on the left (respectively, in the middle; on the right) is that determined by $s_{N}$ (respectively, $\left.\left.s_{1}\right|_{\mathfrak{Y}_{N}} ; s_{1}\right)$; the vertical morphisms in the second row of vertical morphisms are the natural morphisms; the vertical composites are the identity morphisms.

(ii) There is a unique action of $\underline{\Pi}_{X}^{\mathrm{tp}}$ on $\mathfrak{L}_{N} \otimes_{\mathcal{O}_{K_{N}}} \mathcal{O}_{J_{N}}$ [a line bundle on $\left.\mathfrak{Y}_{N} \times_{\mathcal{O}_{K_{N}}} \mathcal{O}_{J_{N}}\right]$ that is compatible with the morphism $\mathfrak{Z}_{N} \rightarrow \mathbb{V}\left(\mathfrak{L}_{N} \otimes_{\mathcal{O}_{K_{N}}} \mathcal{O}_{J_{N}}\right)$ 
determined by $s_{N}$ [hence induces the identity on $\left.s_{N}^{\otimes N}=\left.s_{1}\right|_{3_{N}}\right]$. Moreover, this action of $\underline{\Pi}_{X}^{\mathrm{tp}}$ factors through $\underline{\Pi}_{X}^{\mathrm{tp}} / \underline{\Pi}_{Z_{N}}^{\mathrm{tp}}=\operatorname{Gal}\left(Z_{N} / X\right)$, and, in fact, induces a faithful action of $\underline{\Delta}_{X}^{\mathrm{tp}} / \underline{\Delta}_{Z_{N}}^{\mathrm{tp}}$ on $\mathfrak{L}_{N} \otimes_{\mathcal{O}_{K_{N}}} \mathcal{O}_{J_{N}}$.

Proof. First, observe that by the discussion above [concerning the structure of the special fiber of $\left.\mathfrak{Y}_{N}\right]$, it follows that the action of $\underline{\Pi}_{X}^{\mathrm{tp}}\left(\rightarrow \operatorname{Gal}\left(Y_{N} / X\right)\right)$ on $\mathfrak{Y}_{N}$ preserves the isomorphism class of the line bundle $\mathfrak{L}_{N}$, hence also the isomorphism class of the line bundle $\mathfrak{L}_{N}^{\otimes N}$ [i.e., "the identification of $\mathfrak{L}_{N}^{\otimes N}$ with $\left.\mathfrak{L}_{1}\right|_{\mathfrak{Y}_{N}}$, up to multiplication by an element of $\Gamma\left(\mathfrak{Y}_{N}, \mathcal{O}_{\mathfrak{Y}_{N}}^{\times}\right)=\mathcal{O}_{K_{N}}^{\times}$"]. In particular, if we denote by $\mathcal{G}_{N}$ the group of automorphisms of the pull-back of $\mathfrak{L}_{N}$ to $Y_{N} \times_{K_{N}} J_{N}$ that lie over the $J_{N}$-linear automorphisms of $Y_{N} \times_{K_{N}} J_{N}$ induced by elements of $\underline{\Delta}_{X}^{\mathrm{tp}} / \Delta_{Y_{N}}^{\mathrm{tp}} \subseteq \operatorname{Gal}\left(Y_{N} / X\right)$ and whose $N$-th tensor power fixes the pull-back of $\left.s_{1}\right|_{\mathfrak{Y}_{N}}$, then one verifies immediately [by recalling the definition of $J_{N}$ ] that $\mathcal{G}_{N}$ fits into a natural exact sequence

$$
1 \rightarrow \boldsymbol{\mu}_{N}\left(J_{N}\right) \rightarrow \mathcal{G}_{N} \rightarrow \underline{\Delta}_{X}^{\mathrm{tp}} / \underline{\Delta}_{Y_{N}}^{\mathrm{tp}} \rightarrow 1
$$

- where $\boldsymbol{\mu}_{N}\left(J_{N}\right) \subseteq J_{N}^{\times}$denotes the group of $N$-th roots of unity in $J_{N}$.

Now I claim that the kernel $\mathcal{H}_{N} \subseteq \mathcal{G}_{N}$ of the composite surjection

$$
\mathcal{G}_{N} \rightarrow \underline{\Delta}_{X}^{\mathrm{tp}} / \underline{\Delta}_{Y_{N}}^{\mathrm{tp}} \rightarrow \underline{\Delta}_{X}^{\mathrm{tp}} / \underline{\Delta}_{Y}^{\mathrm{tp}} \cong \underline{\mathbb{Z}}
$$

- where we note that $\operatorname{Ker}\left(\underline{\Delta}_{X}^{\mathrm{tp}} / \underline{\Delta}_{Y_{N}}^{\mathrm{tp}} \rightarrow \underline{\Delta}_{X}^{\mathrm{tp}} / \underline{\Delta}_{Y}^{\mathrm{tp}}\right)=\underline{\Delta}_{Y}^{\mathrm{tp}} / \underline{\Delta}_{Y_{N}}^{\mathrm{tp}} \cong \mathbb{Z} / N \mathbb{Z}(1)$

- is an abelian group annihilated by multiplication by $N$. Indeed, one verifies immediately, by considering various relevant line bundles on " $\mathbb{G}_{\mathrm{m}}$ ", that [if we write $U$ for the standard multiplicative coordinate on $\mathbb{G}_{m}$ and $\zeta$ for a primitive $N$-th root of unity, then] this follows from the identity of functions on " $\mathbb{G}_{\mathrm{m}}$ "

$$
\prod_{j=0}^{N-1} f\left(\zeta^{-j} \cdot U\right)=1
$$

— where $f(U) \stackrel{\text { def }}{=}(U-1) /(U-\zeta)$ represents an element of $\mathcal{H}_{N}$ that maps to a generator of $\Delta_{Y}^{\mathrm{tp}} / \underline{\Delta}_{Y_{N}}^{\mathrm{tp}}$.

Now consider the tautological $\mathbb{Z} / N \mathbb{Z}(1)$-torsor $\mathfrak{R}_{N} \rightarrow \mathfrak{Y}_{N}$ obtained by extracting an $N$-th root of $s_{1}$. More explicitly, $\mathfrak{R}_{N} \rightarrow \mathfrak{Y}_{N}$ may be thought of as the finite $\mathfrak{Y}_{N}$-scheme associated to the $\mathcal{O}_{\mathfrak{Y}_{N}}$-algebra

$$
\bigoplus_{j=0}^{N-1} \mathfrak{L}_{N}^{\otimes-j}
$$

where the "algebra structure" is defined by the morphism $\mathfrak{L}_{N}^{\otimes-N} \rightarrow \mathcal{O}_{\mathfrak{Y}_{N}}$ given by multiplying by $\left.s_{1}\right|_{\mathfrak{Y}_{N}}$. In particular, it follows immediately from the definition of $\mathcal{G}_{N}$ that $\mathcal{G}_{N}$ acts naturally on $\left(\mathfrak{R}_{N}\right)_{J_{N}} \stackrel{\text { def }}{=} \mathfrak{R}_{N} \times_{\mathcal{O}_{K_{N}}} J_{N}$. Since $\left.s_{1}\right|_{Y_{N}}$ 
has zeroes of order 1 at each of the cusps of $Y_{N}$, it thus follows immediately that $\left(\mathfrak{R}_{N}\right)_{J_{N}}$ is connected and Galois over $X_{J_{N}} \stackrel{\text { def }}{=} X \times_{K} J_{N}$, and that one has an isomorphism

$$
\mathcal{G}_{N} \stackrel{\sim}{\rightarrow} \operatorname{Gal}\left(\left(\Re_{N}\right)_{J_{N}} / X_{J_{N}}\right)
$$

arising from the natural action of $\mathcal{G}_{N}$ on $\left(\mathfrak{R}_{N}\right)_{J_{N}}$. Since the abelian group $\underline{\Delta}_{X}^{\mathrm{tp}} / \underline{\Delta}_{Y_{N}}^{\mathrm{tp}}$ acts trivially on $\boldsymbol{\mu}_{N}\left(J_{N}\right)$, and $\mathcal{H}_{N}$ is annihilated by $N$, it thus follows formally from the definition of $\left(\underline{\Delta}_{X}\right)^{\Theta}$ [i.e., as the quotient by a certain "double commutator subgroup"] that at least "geometrically", there exists a map from $\mathfrak{Z}_{N}$ to $\mathfrak{R}_{N}$. More precisely, there is a morphism $\mathfrak{Z}_{N} \times \mathcal{O}_{J_{N}} \bar{K} \rightarrow \mathfrak{R}_{N}$ over $\mathfrak{Y}_{N}$. That this morphism in fact factors through $\mathfrak{Z}_{N}$ - inducing an isomorphism

$$
\mathfrak{Z}_{N} \stackrel{\sim}{\rightarrow} \mathfrak{R}_{N} \times \mathcal{O}_{K_{N}} \mathcal{O}_{J_{N}}
$$

- follows from the definition of the open immersion $G_{J_{N}} \hookrightarrow\left(\underline{\Pi}_{Y_{N}}^{\mathrm{tp}}\right)^{\Theta} / N \cdot\left(\underline{\Delta}_{Y}^{\mathrm{tp}}\right)^{\Theta}$ whose image was used to define $\mathfrak{Z}_{N} \rightarrow \mathfrak{Y}_{N}$ [together with the fact that $\left.s_{1}\right|_{Y_{N}}$ is defined over $\left.K_{N}\right]$. This completes the proof of assertion (i).

Next, we consider assertion (ii). Since the natural action of $\underline{\Pi}_{X}^{\mathrm{tp}}(\rightarrow$ $\left.\operatorname{Gal}\left(Y_{N} / X\right)\right)$ on $\left.\mathfrak{L}_{1}\right|_{\mathfrak{Y}_{N}} \cong \mathfrak{L}_{N}^{\otimes N}$ preserves $\left.s_{1}\right|_{\mathfrak{Y}_{N}}$, and the action of $\underline{\Pi}_{X}^{\mathrm{tp}}$ on $\mathfrak{Y}_{N}$ preserves the isomorphism class of the line bundle $\mathfrak{L}_{N}$, the existence and uniqueness of the desired action of $\underline{\Pi}_{X}^{\mathrm{tp}}$ on $\mathfrak{L}_{N} \otimes_{\mathcal{O}_{K_{N}}} \mathcal{O}_{J_{N}}$ follow immediately from the definitions [cf. especially the definition of $J_{N}$ ]. Moreover, since $s_{N}$ is defined over $Z_{N}$, it is immediate that this action factors through $\underline{\Pi}_{X}^{\mathrm{tp}} / \underline{\Pi}_{Z_{N}}^{\mathrm{tp}}$. Finally, the asserted faithfulness follows from the fact that $s_{1}$ has zeroes of order 1 at the cusps of $Y_{N}$ [together with the tautological fact that $\Delta_{X}^{\text {tp }} / \Delta_{Y_{N}}^{\text {tp }}$ acts faithfully on $\left.Y_{N}\right]$.

Next, let us set

$$
\begin{gathered}
\ddot{K}_{N} \stackrel{\text { def }}{=} K_{2 N} ; \quad \ddot{J}_{N} \stackrel{\text { def }}{=} \ddot{K}_{N}\left(a^{1 / N}\right)_{a \in \ddot{K}_{N}} \subseteq \bar{K} \\
\ddot{\mathfrak{Y}}_{N} \stackrel{\text { def }}{=} \mathfrak{Y}_{2 N} \times \mathcal{O}_{\ddot{K}_{N}} \mathcal{O}_{\ddot{J}_{N}} ; \quad \ddot{Y}_{N} \stackrel{\text { def }}{=} Y_{2 N} \times_{\ddot{K}_{N}} \ddot{J}_{N} ;\left.\quad \ddot{\mathfrak{L}}_{N} \stackrel{\text { def }}{=} \mathfrak{L}_{N}\right|_{\ddot{\mathfrak{Y}}_{N}} \cong \mathfrak{L}_{2 N}^{\otimes 2} \otimes_{\mathcal{O}_{\ddot{K}_{N}}} \mathcal{O}_{\ddot{J}_{N}}
\end{gathered}
$$

and write $\ddot{Z}_{N}$ for the composite of the coverings $\ddot{Y}_{N}, Z_{N}$ of $Y_{N} ; \ddot{\mathfrak{Z}}_{N}$ for the normalization of $\mathfrak{Z}_{N}$ in $\ddot{Z}_{N} ; \ddot{Y} \stackrel{\text { def }}{=} \ddot{Y}_{1}=Y_{2} ; \ddot{\mathfrak{Y}} \stackrel{\text { def }}{=} \ddot{\mathfrak{Y}}_{1}=\mathfrak{Y}_{2} ; \ddot{K} \stackrel{\text { def }}{=} \ddot{K}_{1}=\ddot{J}_{1}=K_{2}$. Thus, we have a cartesian commutative diagram

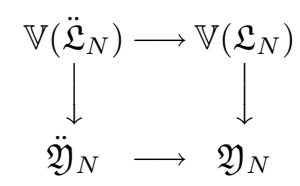

— where, $\underline{\Pi}_{X}^{\mathrm{tp}}$ acts compatibly on $\ddot{\mathfrak{Y}}_{N}, \mathfrak{Y}_{N}$, and [by Proposition 1.1 , (ii)] on $\mathfrak{L}_{N} \otimes_{\mathcal{O}_{K_{N}}} \mathcal{O}_{J_{N}}$. Thus, since this diagram is cartesian [and $J_{N} \subseteq \ddot{J}_{N}$ ], we obtain 
a natural action of $\underline{\Pi}_{X}^{\mathrm{tp}}$ on $\ddot{\mathfrak{L}}_{N}$ which factors through $\underline{\Pi}_{X}^{\mathrm{tp}} / \underline{\Pi}_{\ddot{Z}_{N}}^{\mathrm{tp}}$. Moreover, we have a natural exact sequence

$$
1 \rightarrow \underline{\Pi}_{Z_{N}}^{\mathrm{tp}} / \underline{\Pi}_{\tilde{Z}_{N}}^{\mathrm{tp}} \rightarrow \underline{\Pi}_{Y}^{\mathrm{tp}} / \underline{\Pi}_{\tilde{Z}_{N}}^{\mathrm{tp}} \rightarrow \operatorname{Gal}\left(Z_{N} / Y\right) \rightarrow 1
$$

- where $\underline{\Pi}_{Z_{N}}^{\mathrm{tp}} / \underline{\Pi}_{\ddot{Z}_{N}}^{\mathrm{tp}} \hookrightarrow \operatorname{Gal}\left(\ddot{Y}_{N} / Y_{N}\right)$ - which is compatible with the conjugation actions by $\underline{\Pi}_{X}^{\mathrm{tp}}$ on each of the terms in the exact sequence.

Next, let us choose an orientation on the dual graph of the special fiber of $\mathfrak{Y}$. Such an orientation determines a specific isomorphism $\underline{\mathbb{Z}} \stackrel{\sim}{\rightarrow} \mathbb{Z}$, hence a label $\in \mathbb{Z}$ for each irreducible component of the special fiber of $\mathfrak{Y}$. Note that this choice of labels also determines a label $\in \mathbb{Z}$ for each irreducible component of the special fiber of $\mathfrak{Y}_{N}, \ddot{\mathfrak{Y}}_{N}$. Now we define $\mathfrak{D}_{N}$ to be the effective divisor on $\ddot{\mathfrak{Y}}_{N}$ which is supported on the special fiber and corresponds to the function

$$
\mathbb{Z} \ni j \mapsto j^{2} \cdot \log \left(q_{X}\right) / 2 N
$$

- i.e., at the irreducible component labeled $j$, the divisor $\mathfrak{D}_{N}$ is equal to the divisor given by the schematic zero locus of $q_{X}^{j^{2} / 2 N}$. Note that since the completion of $\ddot{\mathfrak{Y}}_{N}$ at each node of its special fiber is isomorphic to the ring

$$
\mathcal{O}_{\ddot{J}_{N}}[[u, v]] /\left(u v-q_{X}^{1 / 2 N}\right)
$$

- where $u, v$ are indeterminates - it follows that this divisor $\mathfrak{D}_{N}$ is Cartier. Moreover, a simple calculation of degrees reveals that we have an isomorphism of line bundles on $\ddot{\mathfrak{Y}}_{N}$

$$
\mathcal{O}_{\ddot{\mathfrak{y}}_{N}}\left(\mathfrak{D}_{N}\right) \cong \ddot{\mathfrak{L}}_{N}
$$

—i.e., there exists a section, well-defined up to an $\mathcal{O}_{\ddot{J}_{N}}^{\times}$-multiple, $\in \Gamma\left(\ddot{\mathfrak{Y}}_{N}, \ddot{\mathfrak{L}}_{N}\right)$ whose zero locus is precisely the divisor $\mathfrak{D}_{N}$. That is to say, we have a commutative diagram

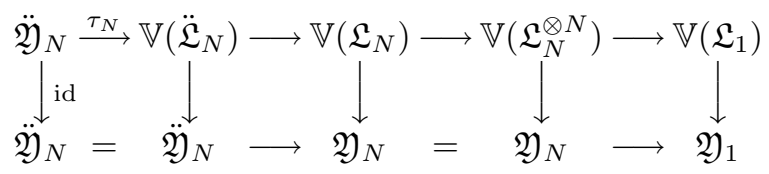

in which the second square is the cartesian commutative diagram discussed above; the third and fourth squares are the lower second and third squares of the diagram of Proposition 1.1, (i); $\tau_{N}$ - which we shall refer to as the theta trivialization of $\ddot{\mathfrak{L}}_{N}$ - is a section whose zero locus is equal to $\mathfrak{D}_{N}$. Moreover, since the action of $\underline{\Pi}_{Y}^{\text {tp }}$ on $\ddot{\mathfrak{Y}}_{N}$ clearly fixes the divisor $\mathfrak{D}_{N}$, we conclude that the action of $\underline{\Pi}_{Y}^{\mathrm{tp}}$ on $\ddot{\mathfrak{Y}}_{N}, \mathbb{V}\left(\ddot{\mathfrak{L}}_{N}\right)$ always preserves $\tau_{N}$, up to an $\mathcal{O}_{\ddot{J}_{N}}^{\times}$-multiple. 
Next, let $M \geq 1$ be an integer that divides $N$. Then $\mathfrak{Y}_{M} \rightarrow \mathfrak{Y}$ (respectively, $\left.\mathfrak{Z}_{M} \rightarrow \mathfrak{Y} ; \ddot{\mathfrak{Y}}_{M} \rightarrow \mathfrak{Y}\right)$ may be regarded as a subcovering of $\mathfrak{Y}_{N} \rightarrow \mathfrak{Y}$ (respectively, $\mathfrak{Z}_{N} \rightarrow \mathfrak{Y} ; \ddot{\mathfrak{Y}}_{N} \rightarrow \mathfrak{Y}$ ). Moreover, we have natural isomorphisms $\left.\mathfrak{L}_{M}\right|_{\mathfrak{Y}_{N}} \cong \mathfrak{L}_{N}^{\otimes N / M} ;\left.\ddot{\mathfrak{L}}_{M}\right|_{\ddot{\mathfrak{Y}}_{N}} \cong \ddot{\mathfrak{L}}_{N}^{\otimes N / M}$. Thus, we obtain a diagram

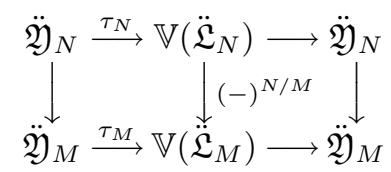

in which the second square consists of the natural morphisms, hence commutes; the first square "commutes up to an $\mathcal{O}_{\ddot{J}_{N}}^{\times}-, \mathcal{O}_{\ddot{J}_{M}}^{\times}-$multiple", i.e., commutes up to composition, at the upper right-hand corner of the square, with an automorphism of $\mathbb{V}\left(\ddot{\mathfrak{L}}_{N}\right)$ arising from multiplication by an element of $\mathcal{O}_{\ddot{J}_{N}}^{\times}$, and, at the lower right-hand corner of the square, with an automorphism of $\mathbb{V}\left(\ddot{\mathfrak{L}}_{M}\right)$ arising from multiplication by an element of $\mathcal{O}_{\ddot{J}_{M}}^{\times}$. [Indeed, this last commutativity follows from the definition of $\ddot{J}_{N}$, and the easily verified fact that there exist " $\tau_{N}$ 's" which are defined over $\mathfrak{Y}_{2 N}$.]

Thus, since by the classical theory of the theta function [cf., e.g., [Mumf], pp. 306-307; the relation " $\ddot{\Theta}(-\ddot{U})=-\ddot{\Theta}(\ddot{U})$ " given in Proposition 1.4, (ii), below], it follows that one may choose $\tau_{1}$ so that the natural action of $\underline{\Pi}_{Y}^{\text {tp }}$ on $\mathbb{V}\left(\ddot{\mathfrak{L}}_{1}\right)$ [arising from the fact that $\ddot{\mathfrak{L}}_{1}$ is the pull-back of the line bundle $\mathfrak{L}_{1}$ on $\mathfrak{Y}$; cf. the action of Proposition 1.1, (ii)] preserves $\pm \tau_{1}$, we conclude, in light of the definition of $\ddot{J}_{N}$, the following:

Lemma 1.2 (Compatibility of Theta Trivializations). By modifying the various $\tau_{N}$ by suitable $\mathcal{O}_{\ddot{J}_{N}}^{\times}$-multiples, one may assume that $\tau_{N_{1}}^{\otimes N_{1} / N_{2}}=\tau_{N_{2}}$, for all positive integers $N_{1}, N_{2}$ such that $N_{2} \mid N_{1}$. In particular, there exists a compatible system [as $N$ varies over the positive integers] of actions of $\underline{\Pi}_{Y}^{\mathrm{tp}}$ (respectively, $\left.\underline{\Pi}_{\ddot{Y}}^{\mathrm{tp}}\right)$ on $\ddot{\mathfrak{Y}}_{N}, \mathbb{V}\left(\ddot{\mathfrak{L}}_{N}\right)$ which preserve $\tau_{N}$. Finally, each action of this system differs from the action determined by the action of Proposition 1.1, (ii), by multiplication by $a(n) 2 N$-th root of unity (respectively, $N$-th root of unity).

Thus, by taking the $\tau_{N}$ to be as in Lemma 1.2 and applying the natural isomorphism $\underline{\Delta}_{\Theta} \cong \widehat{\mathbb{Z}}(1)$ to the difference between the actions of $\underline{\Pi}_{Y}^{\mathrm{tp}}, \underline{\Pi}_{\ddot{Y}}^{\mathrm{tp}}$ arising from Proposition 1.1, (ii), and Lemma 1.2, we obtain the following:

Proposition 1.3 (The Étale Theta Class). The difference between the natural actions of $\underline{\Pi}_{Y}^{\mathrm{tp}}$ arising from Proposition 1.1, (ii); Lemma 1.2, on 
constant multiples of $\tau_{N}$ determines a cohomology class

$$
\eta_{N}^{\Theta} \in H^{1}\left(\underline{\Pi}_{Y}^{\mathrm{tp}},\left(\frac{1}{2} \mathbb{Z} / N \mathbb{Z}\right)(1)\right) \cong H^{1}\left(\underline{\Pi}_{Y}^{\mathrm{tp}}, \underline{\Delta}_{\Theta} \otimes\left(\frac{1}{2} \mathbb{Z} / N \mathbb{Z}\right)\right)
$$

which arises from a cohomology class $\in H^{1}\left(\underline{\Pi}_{Y}^{\mathrm{tp}} / \underline{\Pi}_{\ddot{Z}_{N}}^{\mathrm{tp}}, \underline{\Delta}_{\Theta} \otimes\left(\frac{1}{2} \mathbb{Z} / N \mathbb{Z}\right)\right)$ whose restriction to

$$
H^{1}\left(\underline{\Delta}_{\tilde{Y}_{N}}^{\mathrm{tp}} / \underline{\underline{Z}}_{\ddot{Z}_{N}}^{\mathrm{tp}}, \underline{\Delta}_{\Theta} \otimes\left(\frac{1}{2} \mathbb{Z} / N \mathbb{Z}\right)\right)=\operatorname{Hom}\left(\underline{\Delta}_{\tilde{Y}_{N}}^{\mathrm{tp}} / \underline{\Delta}_{\ddot{Z}_{N}}^{\mathrm{tp}}, \underline{\Delta}_{\Theta} \otimes\left(\frac{1}{2} \mathbb{Z} / N \mathbb{Z}\right)\right)
$$

is the composite of the natural isomorphism $\underline{\Delta}_{\ddot{Y}_{N}}^{\mathrm{tp}} / \underline{\Delta}_{\ddot{Z}_{N}}^{\mathrm{tp}} \stackrel{\sim}{\rightarrow} \Delta_{\Theta} \otimes \mathbb{Z} / N \mathbb{Z}$ with the natural inclusion $\underline{\Theta}_{\Theta} \otimes(\mathbb{Z} / N \mathbb{Z}) \hookrightarrow \underline{\Delta}_{\Theta} \otimes\left(\frac{1}{2} \mathbb{Z} / N \mathbb{Z}\right)$. Moreover, if we set

$$
\mathcal{O}_{K / \ddot{K}}^{\times} \stackrel{\text { def }}{=}\left\{a \in \mathcal{O}_{\ddot{K}}^{\times} \mid a^{2} \in K\right\}
$$

and regard $\mathcal{O}_{K / \ddot{K}}^{\times}$as acting on $H^{1}\left(\underline{\Pi}_{Y}^{\mathrm{tp}},\left(\frac{1}{2} \mathbb{Z} / N \mathbb{Z}\right)(1)\right)$ via the composite

$$
\mathcal{O}_{K / \ddot{K}}^{\times} \rightarrow H^{1}\left(G_{K},\left(\frac{1}{2} \mathbb{Z} / N \mathbb{Z}\right)(1)\right) \rightarrow H^{1}\left(\underline{\Pi}_{Y}^{\mathrm{tp}},\left(\frac{1}{2} \mathbb{Z} / N \mathbb{Z}\right)(1)\right)
$$

- where the first map is the evident generalization of the Kummer map, which is compatible with the Kummer map $\mathcal{O}_{K}^{\times} \rightarrow H^{1}\left(G_{K},(\mathbb{Z} / N \mathbb{Z})(1)\right)$ relative to the natural inclusion $\mathcal{O}_{K}^{\times} \hookrightarrow \mathcal{O}_{K / \ddot{K}}^{\times}$and the morphism induced on cohomology by the natural inclusion $(\mathbb{Z} / N \mathbb{Z}) \hookrightarrow\left(\frac{1}{2} \mathbb{Z} / N \mathbb{Z}\right)$; the second map is the natural map - then the set of cohomology classes

$$
\mathcal{O}_{K / \ddot{K}}^{\times} \cdot \eta_{N}^{\Theta} \in H^{1}\left(\underline{\Pi}_{Y}^{\mathrm{tp}}, \underline{\Delta}_{\Theta} \otimes\left(\frac{1}{2} \mathbb{Z} / N \mathbb{Z}\right)\right)
$$

is independent of the choices of $s_{1}, s_{N}, \tau_{N}$. In particular, by allowing $N$ to vary among all positive integers, we obtain a set of cohomology classes

$$
\mathcal{O}_{K / \ddot{K}}^{\times} \cdot \eta^{\Theta} \in H^{1}\left(\underline{\Pi}_{Y}^{\mathrm{tp}}, \frac{1}{2} \underline{\Delta}_{\Theta}\right)
$$

each of which is a cohomology class $\in H^{1}\left(\left(\underline{\Pi}_{Y}^{\mathrm{tp}}\right)^{\Theta}, \frac{1}{2} \underline{\Delta}_{\Theta}\right)$ whose restriction to

$$
H^{1}\left(\left(\underline{\Delta}_{Y}^{\mathrm{tp}}\right)^{\Theta}, \frac{1}{2} \underline{\Delta}_{\Theta}\right)=\operatorname{Hom}\left(\left(\underline{\Delta}_{Y}^{\mathrm{tp}}\right)^{\Theta}, \frac{1}{2} \underline{\Delta}_{\Theta}\right)
$$

is the composite of the natural isomorphism $\left(\underline{\Delta}_{Y}^{\mathrm{tp}}\right)^{\Theta} \stackrel{\sim}{\rightarrow} \underline{\Delta}_{\Theta}$ with the natural inclusion $\Delta_{\Theta} \hookrightarrow \frac{1}{2} \underline{\Delta}_{\Theta}$. Moreover, the restricted classes

$$
\left.\mathcal{O}_{K / \ddot{K}}^{\times} \cdot \eta^{\Theta}\right|_{\ddot{Y}} \in H^{1}\left(\underline{\Pi}_{\ddot{Y}}^{\mathrm{tp}}, \frac{1}{2} \underline{\Delta}_{\Theta}\right)
$$


arise naturally from classes

$$
\mathcal{O}_{\ddot{K}}^{\times} \cdot \ddot{\eta}^{\Theta} \in H^{1}\left(\underline{\Pi}_{\ddot{Y}}^{\mathrm{tp}}, \underline{\Delta}_{\Theta}\right)
$$

- where $\mathcal{O}_{\ddot{K}}^{\times}$acts via the composite of the Kummer map $\mathcal{O}_{\ddot{K}}^{\times} \rightarrow H^{1}\left(G_{\ddot{K}}, \underline{\Delta}_{\Theta}\right)$ with the natural map $H^{1}\left(G_{\ddot{K}}, \underline{\Delta}_{\Theta}\right) \rightarrow H^{1}\left(\underline{\Pi}_{\tilde{Y}}^{\text {tp }}, \underline{\Delta}_{\Theta}\right)$ - "without denominators". By abuse of the definite article, we shall refer to any element of the sets $\mathcal{O}_{K / \ddot{K}}^{\times}$. $\eta_{N}^{\Theta}, \mathcal{O}_{K / \ddot{K}}^{\times} \cdot \eta^{\Theta}, \mathcal{O}_{\ddot{K}}^{\times} \cdot \ddot{\eta}^{\Theta}$ as the "étale theta class".

Remark 1.3.1. Note that the denominators " $\frac{1}{2}$ " in Proposition 1.3 are by no means superfluous: Indeed, this follows immediately from the fact that the divisor $\mathfrak{D}_{1}$ on $\ddot{\mathfrak{Y}}$ clearly does not descend to $\mathfrak{Y}$.

Let us denote by

$$
\mathfrak{U} \subseteq \mathfrak{Y}
$$

the open formal subscheme obtained by removing the nodes from the irreducible component of the special fiber labeled $0 \in \mathbb{Z}$. If we take the unique cusp lying in $\mathfrak{U}$ as the origin, then - as is well-known from the theory of the Tate curve [cf., e.g., [Mumf], pp. 306-307] — the group structure on the underlying elliptic curve of $X^{\log }$ determines a group structure on $\mathfrak{U}$, together with a unique [in light of our choice of an orientation on the dual graph of the special fiber of $\mathfrak{Y}]$ isomorphism of $\mathfrak{U}$ with the $p$-adic formal completion of $\mathbb{G}_{\mathrm{m}}$ over $\mathcal{O}_{K}$. In particular, this isomorphism determines a multiplicative coordinate

$$
U \in \Gamma\left(\mathfrak{U}, \mathcal{O}_{\mathfrak{U}}^{\times}\right)
$$

— which, as one verifies immediately from the definitions, admits a square root

$$
\ddot{U} \in \Gamma\left(\ddot{\mathfrak{U}}, \mathcal{O}_{\ddot{\mathfrak{U}}}^{\times}\right)
$$

on $\ddot{\mathfrak{U}} \stackrel{\text { def }}{=} \mathfrak{U} \times \mathfrak{Y} \ddot{\mathfrak{Y}}$.

Proposition 1.4 (Relation to the Classical Theta Function). Set

$$
\ddot{\Theta}=\ddot{\Theta}(\ddot{U}) \stackrel{\text { def }}{=} q_{X}^{-\frac{1}{8}} \cdot \sum_{n \in \mathbb{Z}}(-1)^{n} \cdot q_{X}^{\frac{1}{2}\left(n+\frac{1}{2}\right)^{2}} \cdot \ddot{U}^{2 n+1} \in \Gamma\left(\ddot{\mathfrak{U}}, \mathcal{O}_{\ddot{\mathfrak{U}}}\right)
$$

so $\ddot{\Theta}$ extends uniquely to a meromorphic function on $\ddot{\mathfrak{Y}}[c f$., e.g., [Mumf], pp. 306-307]. Then:

(i) The zeroes of $\ddot{\Theta}$ on $\ddot{\mathfrak{Y}}$ are precisely the cusps of $\ddot{\mathfrak{Y}}$; each zero has multiplicity 1. The divisor of poles of $\ddot{\Theta}$ on $\ddot{\mathfrak{Y}}$ is precisely the divisor $\mathfrak{D}_{1}$. 
(ii) We have

$$
\begin{aligned}
& \ddot{\Theta}(\ddot{U})=-\ddot{\Theta}\left(\ddot{U}^{-1}\right) ; \quad \ddot{\Theta}(-\ddot{U})=-\ddot{\Theta}(\ddot{U}) \\
& \ddot{\Theta}\left(q_{X}^{a / 2} \ddot{U}\right)=(-1)^{a} \cdot q_{X}^{-a^{2} / 2} \cdot \ddot{U}^{-2 a} \cdot \ddot{\Theta}(\ddot{U})
\end{aligned}
$$

for $a \in \mathbb{Z}$.

(iii) The classes

$$
\mathcal{O}_{\ddot{K}}^{\times} \cdot \ddot{\eta}^{\Theta} \in H^{1}\left(\underline{\Pi}_{\ddot{Y}}^{\mathrm{tp}}, \underline{\Delta}_{\Theta}\right)
$$

of Proposition 1.3 are precisely the "Kummer classes" associated to $\mathcal{O}_{\ddot{K}}^{\times}$multiples of $\ddot{\Theta}$, regarded as a regular function on $\ddot{Y}$. In particular, if $L$ is a finite extension of $\ddot{K}$, and $y \in \ddot{Y}(L)$ is a non-cuspidal point, then the restricted classes

$$
\left.\mathcal{O}_{\ddot{K}}^{\times} \cdot \ddot{\eta}^{\Theta}\right|_{y} \in H^{1}\left(G_{L}, \underline{\Delta}_{\Theta}\right) \cong H^{1}\left(G_{L}, \widehat{\mathbb{Z}}(1)\right) \cong\left(L^{\times}\right)^{\wedge}
$$

- where the " $\wedge$ " denotes the profinite completion - lie in $L^{\times} \subseteq\left(L^{\times}\right)^{\wedge}$ and are equal to the values $\mathcal{O}_{\ddot{K}}^{\times} \cdot \ddot{\Theta}(y)$ of $\ddot{\Theta}$ and its $\mathcal{O}_{\ddot{K}}^{\times}$-multiples at $y$. A similar statement holds if $y \in \ddot{Y}(L)$ is a cusp, if one restricts first to the associated decomposition group $D_{y}$ and then to a section $G_{L} \hookrightarrow D_{y}$ compatible with the canonical integral structure [cf. [Mzk13], Definition 4.1, (iii)] on $D_{y}$. In light of this relationship between the cohomology classes of Proposition 1.3 and the values of $\ddot{\Theta}$, we shall sometimes refer to these classes as "the étale theta function".

Proof. Assertion (ii) is a routine calculation involving the series used to define $\ddot{\Theta}$. A similar calculation shows that $\ddot{\Theta}( \pm 1)=0$. The formula given for $\ddot{\Theta}\left(q_{X}^{a / 2} \ddot{U}\right)$ in assertion (ii) shows that the portion of the divisor of poles supported in the special fiber of $\ddot{\mathfrak{Y}}$ is equal to $\mathfrak{D}_{1}$. This formula also shows that to complete the proof of assertion (i), it suffices to show that the given description of the zeroes and poles of $\ddot{\Theta}$ is accurate over the irreducible component of the special fiber of $\ddot{\mathfrak{Y}}$ labeled 0 . But this follows immediately from the fact that the restriction of $\ddot{\Theta}$ to this irreducible component is the rational function $\ddot{U}-\ddot{U}^{-1}$. Finally, in light of assertion (i) [and the fact, observed above, that $\left.\Gamma\left(\mathfrak{Y}_{N}, \mathcal{O}_{\mathfrak{Y}_{N}}^{\times}\right)=\mathcal{O}_{K_{N}}^{\times}\right]$, assertion (iii) is a formal consequence of the construction of the classes $\mathcal{O}_{\ddot{K}}^{\times} \cdot \ddot{\eta}^{\Theta}$.

Proposition 1.5 (Theta Cohomology).

(i) The Leray-Serre spectral sequences associated to the filtration of closed subgroups

$$
\underline{\Delta}_{\Theta} \subseteq\left(\underline{\Delta}_{Y}^{\mathrm{tp}}\right)^{\Theta} \subseteq\left(\underline{\Pi}_{Y}^{\mathrm{tp}}\right)^{\Theta}
$$


determine a natural filtration $0 \subseteq F^{2} \subseteq F^{1} \subseteq F^{0}=H^{1}\left(\left(\underline{\Pi}_{Y}^{\mathrm{tp}}\right)^{\Theta}, \underline{\Delta}_{\Theta}\right)$ on the cohomology module $H^{1}\left(\left(\underline{\Pi}_{Y}^{\mathrm{tp}}\right)^{\Theta}, \underline{\Delta}_{\Theta}\right)$ with subquotients

$$
\begin{gathered}
F^{0} / F^{1}=\operatorname{Hom}\left(\underline{\Delta}_{\Theta}, \underline{\Delta}_{\Theta}\right)=\widehat{\mathbb{Z}} \cdot \log (\Theta) \\
F^{1} / F^{2}=\operatorname{Hom}\left(\left(\underline{\Delta}_{Y}^{\text {tp }}\right)^{\text {ell }} / \underline{\Delta}_{\Theta}, \underline{\Delta}_{\Theta}\right)=\widehat{\mathbb{Z}} \cdot \log (U) \\
F^{2}=H^{1}\left(G_{K}, \underline{\Delta}_{\Theta}\right) \stackrel{\sim}{\rightarrow} H^{1}\left(G_{K}, \widehat{\mathbb{Z}}(1)\right) \stackrel{\sim}{\rightarrow}\left(K^{\times}\right)^{\wedge}
\end{gathered}
$$

- where we use the symbol $\log (\Theta)$ to denote the identity morphism $\underline{\Delta}_{\Theta} \rightarrow \underline{\Delta}_{\Theta}$ and the symbol $\log (U)$ to denote the standard isomorphism $\left(\underline{\Delta}_{Y}^{\mathrm{tp}}\right)^{\mathrm{ell}} / \underline{\Delta}_{\Theta} \stackrel{\sim}{\rightarrow}$ $\widehat{\mathbb{Z}}(1) \stackrel{\sim}{\rightarrow} \underline{\Delta}_{\Theta}$.

(ii) Similarly, the Leray-Serre spectral sequences associated to the filtration of closed subgroups

$$
\underline{\Delta}_{\Theta} \subseteq\left(\underline{\Delta}_{\ddot{Y}}^{\mathrm{tp}}\right)^{\Theta} \subseteq\left(\underline{\Pi}_{\ddot{Y}}^{\mathrm{tp}}\right)^{\Theta}
$$

determine a natural filtration $0 \subseteq \ddot{F}^{2} \subseteq \ddot{F}^{1} \subseteq \ddot{F}^{0}=H^{1}\left(\left(\underline{\Pi}_{\ddot{Y}}^{\mathrm{tp}}\right)^{\Theta}, \underline{\Delta}_{\Theta}\right)$ on the cohomology module $H^{1}\left(\left(\underline{\Pi}_{\tilde{Y}}^{\mathrm{tp}}\right)^{\Theta}, \underline{\Delta}_{\Theta}\right)$ with subquotients

$$
\begin{gathered}
\ddot{F}^{0} / \ddot{F}^{1}=\operatorname{Hom}\left(\underline{\Delta}_{\Theta}, \Delta_{\Theta}\right)=\widehat{\mathbb{Z}} \cdot \log (\Theta) \\
\ddot{F}^{1} / \ddot{F}^{2}=\operatorname{Hom}\left(\left(\underline{\Delta}_{\ddot{Y}}^{\text {tp }}\right)^{\text {ell }} / \underline{\Delta}_{\Theta}, \underline{\Delta}_{\Theta}\right)=\overleftrightarrow{\mathbb{Z}} \cdot \log (\ddot{U}) \\
\ddot{F}^{2}=H^{1}\left(G_{\ddot{K}}, \underline{\Delta}_{\Theta}\right) \stackrel{\sim}{\rightarrow} H^{1}\left(G_{\ddot{K}}, \widehat{\mathbb{Z}}(1)\right) \stackrel{\sim}{\rightarrow}\left(\ddot{K}^{\times}\right)^{\wedge}
\end{gathered}
$$

- where we write $\log (\ddot{U}) \stackrel{\text { def }}{=} \frac{1}{2} \cdot \log (U)$.

(iii) Any class $\ddot{\eta}^{\Theta} \in H^{1}\left(\underline{\Pi}_{\ddot{Y}}^{\mathrm{tp}}, \underline{\Delta}_{\Theta}\right)$ arises from a unique class [which, by abuse of notation, we shall denote by] $\ddot{\eta}^{\Theta} \in H^{1}\left(\left(\underline{\Pi}_{\ddot{Y}}^{\mathrm{tp}}\right)^{\Theta}, \underline{\Delta}_{\Theta}\right)$ that maps to $\log (\Theta)$ in the quotient $\ddot{F}^{0} / \ddot{F}^{1}$ and on which $a \in \mathbb{Z} \cong \underline{\mathbb{Z}} \cong \underline{\Pi}_{X}^{\mathrm{tp}} / \underline{\Pi}_{Y}^{\mathrm{tp}}$ acts as follows:

$$
\ddot{\eta}^{\Theta} \mapsto \ddot{\eta}^{\Theta}-2 a \cdot \log (\ddot{U})-\frac{a^{2}}{2} \cdot \log \left(q_{X}\right)+\log \left(\mathcal{O}_{\ddot{K}}^{\times}\right)
$$

- where we use the notation "log" to express the fact that we wish to write the group structure of $\left(\ddot{K}^{\times}\right)^{\wedge}$ additively. Similarly, any inversion automorphism $\iota$ of $\underline{\Pi}_{Y}^{\mathrm{tp}}$ - i.e., an automorphism lying over the action of " -1 " on the underlying elliptic curve of $X^{\log }$ which fixes the irreducible component of the special fiber of $\mathfrak{Y}$ labeled 0 - fixes $\ddot{\eta}^{\Theta}+\log \left(\mathcal{O}_{\ddot{K}}^{\times}\right)$, but maps $\log (\ddot{U})+\log \left(\mathcal{O}_{\ddot{K}}^{\times}\right)$ to $-\log (\ddot{U})+\log \left(\mathcal{O}_{\ddot{K}}^{\times}\right)$.

Proof. Assertions (i), (ii) follow immediately from the definitions. Here, in (i) (respectively, (ii)), we note that the fact that $F^{0}$ (respectively, $\ddot{F}^{0}$ ) surjects onto $\operatorname{Hom}\left(\underline{\Delta}_{\Theta}, \underline{\Delta}_{\Theta}\right)$ follows, for instance, by considering the Kummer class of the meromorphic function $\ddot{\Theta} \cdot \ddot{U}^{-1}$ on $\mathfrak{Y}$ (respectively, $\ddot{\Theta}$ on $\ddot{\mathfrak{Y}}$ - cf. Proposition 1.4, (iii)). Assertion (iii) follows from Propositions 1.3; 1.4, (ii), (iii). 
Theorem 1.6 (Tempered Anabelian Rigidity of the Étale Theta Function). $\quad$ Let $X_{\alpha}^{\log }$ (respectively, $X_{\beta}^{\log }$ ) be a smooth log curve of type $(1,1)$ over a finite extension $K_{\alpha}$ (respectively, $K_{\beta}$ ) of $\mathbb{Q}_{p}$; we assume that $X_{\alpha}^{\log }$ (respectively, $X_{\beta}^{\log }$ ) has stable reduction over $\mathcal{O}_{K_{\alpha}}$ (respectively, $\mathcal{O}_{K_{\beta}}$ ), and that the resulting special fibers are singular and split. We shall use similar notation for objects associated to $X_{\alpha}^{\log }, X_{\beta}^{\log }$ [but with a subscript $\alpha$ or $\beta$ ] to the notation that was used for objects associated to $X^{\log }$. Let

$$
\gamma: \underline{\Pi}_{X_{\alpha}}^{\mathrm{tp}} \stackrel{\sim}{\rightarrow} \underline{\Pi}_{X_{\beta}}^{\mathrm{tp}}
$$

be an isomorphism of topological groups. Then:

(i) We have: $\gamma\left(\underline{\Pi}_{\ddot{Y}_{\alpha}}^{\mathrm{tp}}\right)=\underline{\Pi}_{\ddot{Y}_{\beta}}^{\mathrm{tp}}$.

(ii) $\gamma$ induces an isomorphism

$$
\left(\underline{\Delta}_{\Theta}\right)_{\alpha} \stackrel{\sim}{\rightarrow}\left(\underline{\Delta}_{\Theta}\right)_{\beta}
$$

that is compatible with the surjections

$$
\begin{aligned}
H^{1}\left(G_{\ddot{K}_{\alpha}},\left(\underline{\Delta}_{\Theta}\right)_{\alpha}\right) \stackrel{\sim}{\rightarrow} H^{1}\left(G_{\ddot{K}_{\alpha}}, \widehat{\mathbb{Z}}(1)\right) \stackrel{\sim}{\rightarrow}\left(\ddot{K}_{\alpha}^{\times}\right)^{\wedge} \rightarrow \widehat{\mathbb{Z}} \\
H^{1}\left(G_{\ddot{K}_{\beta}},\left(\underline{\Delta}_{\Theta}\right)_{\beta}\right) \stackrel{\sim}{\rightarrow} H^{1}\left(G_{\ddot{K}_{\beta}}, \widehat{\mathbb{Z}}(1)\right) \stackrel{\sim}{\rightarrow}\left(\ddot{K}_{\beta}^{\times}\right)^{\wedge} \rightarrow \widehat{\mathbb{Z}}
\end{aligned}
$$

determined by the valuations on $\ddot{K}_{\alpha}, \ddot{K}_{\beta}$. That is to say, $\gamma$ induces an isomorphism $H^{1}\left(G_{\ddot{K}_{\alpha}},\left(\underline{\Delta}_{\Theta}\right)_{\alpha}\right) \stackrel{\sim}{\rightarrow} H^{1}\left(G_{\ddot{K}_{\beta}},\left(\underline{\Delta}_{\Theta}\right)_{\beta}\right)$ that preserves both the kernel of these surjections and the elements " $1 \in \widehat{\mathbb{Z}}$ " in the resulting quotients.

(iii) The isomorphism of cohomology groups induced by $\gamma$ maps the classes

$$
\mathcal{O}_{\ddot{K}_{\alpha}}^{\times} \cdot \ddot{\eta}_{\alpha}^{\Theta} \in H^{1}\left(\underline{\Pi}_{\ddot{Y}_{\alpha}}^{\mathrm{tp}},\left(\underline{\Delta}_{\Theta}\right)_{\alpha}\right)
$$

of Proposition 1.3 for $X_{\alpha}$ to some $\underline{\Pi}_{X_{\beta}}^{\mathrm{tp}} / \underline{\Pi}_{Y_{\beta}}^{\mathrm{tp}} \cong \underline{\mathbb{Z}}$-conjugate of the corresponding classes

$$
\mathcal{O}_{\ddot{K}_{\beta}}^{\times} \cdot \ddot{\eta}_{\beta}^{\Theta} \in H^{1}\left(\underline{\Pi}_{\ddot{Y}_{\beta}}^{\mathrm{tp}},\left(\underline{\Delta}_{\Theta}\right)_{\beta}\right)
$$

of Proposition 1.3 for $X_{\beta}$.

Proof. Assertion (i) is immediate from the definitions; the discreteness of the topological group " $\mathbb{Z}$ "; and the fact that $\gamma$ maps $\Delta_{X_{\alpha}}^{\text {tp }}$ onto $\Delta_{X_{\beta}}^{\text {tp }}[$ cf. [Mzk2], Lemma 1.3.8] and preserves decomposition groups of cusps [cf. [Mzk14], Theorem 6.5, (iii)]. As for assertion (ii), the fact that $\gamma$ induces an isomorphism $\left(\underline{\Delta}_{\Theta}\right)_{\alpha} \stackrel{\sim}{\rightarrow}\left(\underline{\Delta}_{\Theta}\right)_{\beta}$ is immediate [in light of the argument used to verify assertion (i)] from the definitions. The asserted compatibility then follows from [Mzk14], Theorem 6.12; [Mzk2], Proposition 1.2.1, (iv), (vi), (vii). 
Next, we consider assertion (iii). By composing $\gamma$ with an appropriate inner automorphism of $\underline{\Pi}_{X_{\beta}}^{\mathrm{tp}}$, it follows from [Mzk14], Theorem 6.8, (ii), that we may assume that the isomorphism $\underline{\Pi}_{\tilde{Y}_{\alpha}}^{\mathrm{tp}} \stackrel{\sim}{\rightarrow} \underline{\Pi}_{\tilde{Y}_{\beta}}^{\mathrm{tp}}$ is compatible with suitable "inversion automorphisms" $\iota_{\alpha}, \iota_{\beta}$ [cf. Proposition 1.5, (iii)] on both sides. Next, let us observe that it is a tautology that $\gamma$ is compatible with the symbols " $\log (\Theta)$ " of Proposition 1.5, (i), (ii). On the other hand, by Proposition 1.5, (ii), (iii), the property of "mapping to $\log (\Theta)$ in the quotient $\ddot{F}^{0} / \ddot{F}^{1}$ and being fixed, up to a unit multiple, under an inversion automorphism" completely determines the classes $\ddot{\eta}^{\Theta}$ up to a $\left(\ddot{K}^{\times}\right)^{\wedge}$-multiple. Thus, to complete the proof, it suffices to reduce this "indeterminacy up to a $\left(\ddot{K}^{\times}\right)^{\wedge}$-multiple" to an "indeterminacy up to a $\mathcal{O}_{\ddot{K}}^{\times}$-multiple".

This "reduction of indeterminacy from $\left(\ddot{K}^{\times}\right)^{\wedge}$ to $\mathcal{O}_{\ddot{K}}^{\times}$" may be achieved [in light of the compatibility shown in assertion (ii)] by evaluating the classes $\ddot{\eta}^{\Theta}$ at a cusp that maps to the irreducible component of the special fiber of $\ddot{\mathfrak{Y}}$ labeled 0 [e.g., a cusp that is preserved by the inversion automorphism] via the canonical integral structure, as in Proposition 1.4, (iii), and applying the fact that, by [Mzk14], Theorem 6.5, (iii); [Mzk14], Corollary 6.11, $\gamma$ preserves both the decomposition groups and the canonical integral structures [on the decomposition groups] of cusps.

Remark 1.6.1. In the proof of Theorem 1.6, (iii), we eliminated the "indeterminacy" in question by restricting to cusps, via the canonical integral structure. Another way to eliminate this indeterminacy is to restrict to noncuspidal torsion points, which are temp-absolute by [Mzk14], Theorem 6.8, (iii). This latter approach amounts to invoking the theory of [Mzk13], §2, which is, in some sense, less elementary [for instance, in the sense that it makes use, in a much more essential way, of the main result of [Mzk11]] than the theory of [Mzk13], $\$ 4$ [which one is, in effect, applying if one uses cusps].

Remark 1.6.2. One way of thinking about isomorphisms of the tempered fundamental group is that they arise from variation of the basepoint, or underlying set theory, relative to which one considers the associated "temperoids" [cf. [Mzk14], §3]. Indeed, this is the point of view taken in [Mzk12], in the case of anabelioids. From this point of view, the content of Theorem 1.6 may be interpreted as stating that:

The étale theta function is preserved by arbitrary "changes of the underlying set theory" relative to which one considers the tempered fundamental group in question. 
When viewed in this way, Theorem 1.6 may be thought of - especially if one takes the non-cuspidal approach of Remark 1.6.1 — as a sort of nonarchimedean analogue of the so-called functional equation of the classical complex theta function, which also states that the "theta function" is preserved, in effect, by "changes of the underlying set theory" relative to which one considers the integral singular cohomology of the elliptic curve in question, i.e., more concretely, by the action of the modular group $S L_{2}(\mathbb{Z})$. Note that in the complex case, it is crucial, in order to prove the functional equation, to have not only the "Schottky uniformization" $\mathbb{C}^{\times} \rightarrow \mathbb{C}^{\times} / q^{\mathbb{Z}}$ by $\mathbb{C}^{\times}$- which naturally gives rise to the analytic series representation of the theta function, but is not, however, preserved by the action of the modular group - but also the full uniformization of an elliptic curve by $\mathbb{C}$ [which is preserved by the action of the modular group]. This "preservation of the full uniformization by $\mathbb{C}$ " in the complex case may be regarded as being analogous to the preservation of the non-cuspidal torsion points in the approach to proving Theorem 1.6 discussed in Remark 1.6.1.

Remark 1.6.3. The interpretation of Theorem 1.6 given in Remark 1.6.2 is reminiscent of the discussion given in the Introduction of [Mzk7], in which the author expresses his hope, in effect, that some sort of $p$-adic analogue of the functional equation of the theta function could be developed.

Remark 1.6.4. One verifies immediately that there are [easier] profinite versions of the constructions given in the present $\S 1$ : That is to say, if we denote by

$$
\left(\ddot{Y}^{\log }\right)^{\wedge} \rightarrow\left(Y^{\log }\right)^{\wedge} \rightarrow X^{\log }
$$

the profinite étale coverings determined by the tempered coverings $\ddot{Y}^{\log } \rightarrow$ $Y^{\log } \rightarrow X^{\log }\left[\right.$ so $\left.\underline{\Pi}_{X} / \underline{\Pi}_{Y^{\wedge}} \cong \widehat{\mathbb{Z}}\right]$, then the set of classes $\mathcal{O}_{\ddot{K}}^{\times} \cdot \ddot{\eta}^{\Theta} \in H^{1}\left(\underline{\Pi}_{\ddot{Y}}^{\mathrm{tp}}, \underline{\Delta}_{\Theta}\right)$ determines, by profinite completion, a set of classes

$$
\mathcal{O}_{\ddot{K}}^{\times} \cdot\left(\ddot{\eta}^{\Theta}\right)^{\wedge} \in H^{1}\left(\underline{\Pi}_{\ddot{Y}^{\wedge}}, \underline{\Delta}_{\Theta}\right)
$$

on which any $\underline{\Pi}_{X} / \underline{\Pi}_{Y^{\wedge}} \cong \widehat{\mathbb{Z}} \ni a$ acts via

$$
\left(\ddot{\eta}^{\Theta}\right)^{\wedge} \mapsto\left(\ddot{\eta}^{\Theta}\right)^{\wedge}-2 a \cdot \log (\ddot{U})-\frac{a^{2}}{2} \cdot \log \left(q_{X}\right)+\log \left(\mathcal{O}_{\ddot{K}}^{\times}\right)
$$

[cf. Proposition 1.5, (iii)]. Moreover, given $X_{\alpha}, X_{\beta}$ as in Theorem 1.6, any isomorphism

$$
\underline{\Pi}_{X_{\alpha}} \stackrel{\sim}{\rightarrow} \underline{\Pi}_{X_{\beta}}
$$


preserves these profinite étale theta functions.

In fact, it is possible to eliminate the $\mathcal{O}_{\ddot{K}}^{\times}$-indeterminacy of Theorem 1.6, (iii), to a substantial extent [cf. [Mzk13], Corollary 4.12]. For simplicity, let us assume in the following discussion that the following two conditions hold:

(I) $K=\ddot{K}$.

(II) The hyperbolic curve determined by $X^{\log }$ is not arithmetic over $K$

[cf., e.g., [Mzk3], Remark 2.1.1].

As is well-known, condition (II) amounts, relative to the $j$-invariant of the elliptic curve underlying $X$, to the assertion that we exclude four exceptional $j$-invariants [cf. [Mzk3], Proposition 2.7].

Now let us write $\ddot{X}^{\log } \rightarrow X^{\log }$ for the Galois [by condition (I)] covering of degree 4 determined by the "multiplication by 2 " map on the elliptic curve underlying $X$; write $X^{\log } \rightarrow C^{\log }$ for the stack-theoretic quotient of $X^{\log }$ by the natural action of \pm 1 on [the underlying elliptic curve of] $X$. Thus, [by condition (II)] the hyperbolic orbicurve determined by $C^{\log }$ is a $K$-core [cf. [Mzk3], Remark 2.1.1]. Observe that the covering $\ddot{X}^{\log } \rightarrow C^{\log }$ is Galois, with Galois group isomorphic to $(\mathbb{Z} / 2 \mathbb{Z})^{3}$. Moreover, we have two natural automorphisms

$$
\epsilon_{\mu} \in \operatorname{Gal}(\ddot{X} / X) \subseteq \operatorname{Gal}(\ddot{X} / C) ; \quad \epsilon_{ \pm} \in \operatorname{Gal}(\ddot{X} / C)
$$

- i.e., respectively, the unique nontrivial element of $\operatorname{Gal}(\ddot{X} / X)$ that acts trivially on the set of irreducible components of the special fiber; the unique nontrivial element of $\operatorname{Gal}(\ddot{X} / C)$ that acts trivially on the set of cusps of $\ddot{X}$.

Now suppose that we are given a nontrivial element

$$
\epsilon_{\mathbb{Z}} \in \operatorname{Gal}(\ddot{X} / X)
$$

which is $\neq \epsilon_{\mu}$. Then $\epsilon_{\mathbb{Z}}$ determines a commutative diagram

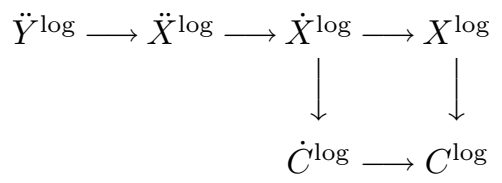

— where $\ddot{Y}^{\log } \rightarrow \ddot{X}^{\log }, X^{\log } \rightarrow C^{\log }$ are the natural morphisms; $\ddot{X}^{\log } \rightarrow \dot{X}^{\log }$ is the quotient by the action of $\epsilon_{\mathbb{Z}} ; \dot{X}^{\log } \rightarrow X^{\log }$ is the quotient by the action of $\epsilon_{\mu} ; \dot{X}^{\log } \rightarrow \dot{C}^{\log }$ is the [stack-theoretic] quotient by the action of $\epsilon_{ \pm} \cdot \epsilon_{\mu}$; the square is cartesian.

Definition 1.7. We shall refer to a smooth log orbicurve over $K$ that arises, up to isomorphism, as the smooth $\log$ orbicurve $\dot{X}^{\log }$ (respectively, $\dot{C}^{\log }$ ) 
constructed above for some choice of $\epsilon_{\mathbb{Z}}$ as being of type $\left(1, \boldsymbol{\mu}_{2}\right)$ (respectively, $\left.\left(1, \boldsymbol{\mu}_{2}\right)_{ \pm}\right)$. We shall also apply this terminology to the associated hyperbolic orbicurves.

Proposition 1.8 (Characteristic Nature of Coverings). $\quad$ For $\square=\alpha, \beta$, let $\dot{X}_{\square}^{\log }$ be a smooth log curve of type $\left(1, \boldsymbol{\mu}_{2}\right)$ over a finite extension $K_{\square}$ of $\mathbb{Q}_{p}$; write $\ddot{Y}_{\square}^{\log }, \ddot{X}_{\square}^{\log }, X_{\square}^{\log }, \dot{C}_{\square}^{\log }, C_{\square}^{\log }$ for the related smooth log orbicurves [as in the above discussion]. Then any isomorphism of topological groups

$$
\gamma: \underline{\Pi}_{\dot{X}_{\alpha}}^{\mathrm{tp}} \stackrel{\sim}{\rightarrow} \underline{\Pi}_{\dot{X}_{\beta}}^{\mathrm{tp}}\left(\text { respectively, } \gamma: \underline{\Pi}_{\dot{C}_{\alpha}}^{\mathrm{tp}} \stackrel{\sim}{\rightarrow} \underline{\Pi}_{\dot{C}_{\beta}}^{\mathrm{tp}}\right)
$$

induces an isomorphism between the commutative diagrams of outer homomorphisms of topological groups

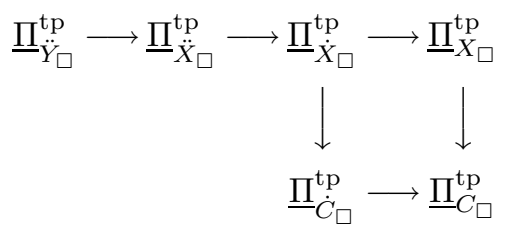

- where $\square=\alpha, \beta$. A similar statement holds when " $\underline{\text { tp }}$ " is replaced by "II".

Proof. First, we consider the tempered case. By [Mzk2], Lemma 1.3.8, it follows that $\gamma$ is compatible with the respective projections to $G_{K_{\square}}$. By [Mzk14], Theorem 6.8, (ii) [cf. also [Mzk3], Theorem 2.4], it follows from condition (II) that $\gamma$ induces an isomorphism $\underline{\Pi}_{C_{\alpha}}^{\mathrm{tp}} \stackrel{\sim}{\rightarrow} \underline{\Pi}_{C_{\beta}}^{\mathrm{tp}}$ that is compatible with $\gamma$. Since [as is easily verified] $\underline{\Delta}_{X_{\square}}^{\mathrm{tp}} \subseteq \underline{\Delta}_{C_{\square}}^{\mathrm{tp}}$ may be characterized as the unique open subgroup of index 2 that corresponds to a double covering which is a scheme [i.e., open subgroup of index 2 whose profinite completion contains no torsion elements - cf., e.g., [Mzk16], Lemma 2.1, (v)], it follows that $\gamma$ determines an isomorphism $\underline{\Delta}_{X_{\alpha}}^{\mathrm{tp}} \stackrel{\sim}{\rightarrow} \underline{\Delta}_{X_{\beta}}^{\mathrm{tp}}$, hence also an isomorphism $\left(\underline{\Delta}_{X_{\alpha}}^{\mathrm{tp}}\right)^{\text {ell }} \stackrel{\sim}{\rightarrow}\left(\underline{\Delta}_{X_{\beta}}^{\mathrm{tp}}\right)^{\text {ell }}$. Moreover, by considering the discreteness of $\operatorname{Gal}\left(Y_{\square} / X_{\square}\right) \cong \underline{\mathbb{Z}}_{\square}$, or, alternatively, the triviality of the action of $G_{K_{\square}}$ on $\operatorname{Gal}\left(Y_{\square} / X_{\square}\right)$, it follows that this last isomorphism determines an isomorphism $\underline{\Delta}_{X_{\alpha}}^{\mathrm{tp}} / \underline{\Delta}_{Y_{\alpha}}^{\mathrm{tp}} \stackrel{\sim}{\rightarrow} \underline{\Delta}_{X_{\beta}}^{\mathrm{tp}} / \underline{\Delta}_{Y_{\beta}}^{\mathrm{tp}} \cong \underline{\mathbb{Z}}_{\beta}$, hence [by considering the kernel of the action of $\underline{\Pi}_{C_{\square}}^{\mathrm{tp}}$ on $\underline{\Delta}_{X_{\square}}^{\mathrm{tp}} / \underline{\Delta}_{Y_{\square}}^{\mathrm{tp}}$ ] an isomorphism $\underline{\Pi}_{X_{\alpha}}^{\mathrm{tp}} \stackrel{\sim}{\rightarrow} \underline{\Pi}_{X_{\beta}}^{\mathrm{tp}}$. Since $\dot{X}_{\square}^{\log } \rightarrow \dot{C}_{\square}^{\mathrm{log}}$ may be characterized as the quotient by the unique automorphism of $\dot{X}_{\square}^{\log }$ over $C_{\square}^{\log }$ that acts nontrivially on the cusps of $\dot{X}_{\square}^{\log }$ [where we recall that $\gamma$ preserves decomposition group of cusps — cf. [Mzk14], Theorem 6.5, (iii)] but does not lie over $X_{\square}^{\log }$, we thus conclude that $\gamma$ induces isomorphisms between the respective $\underline{\Pi}_{\dot{X}_{\square}}^{\mathrm{tp}}, \underline{\Pi}_{\dot{C}_{\square}}^{\mathrm{tp}}, \underline{\Pi}_{X_{\square}}^{\mathrm{tp}}, \underline{\Pi}_{C_{\square}}^{\mathrm{tp}}$ that are compatible with the natural inclusions among these subgroups [for a 
fixed " $\square$ "]. Moreover, since $\gamma$ preserves the decomposition groups of cusps of $\underline{\Pi}_{X_{\square}}^{\text {tp }}$ [cf. [Mzk14], Theorem 6.5, (iii)], we conclude immediately that $\gamma$ is also compatible with the subgroups $\underline{\Pi}_{\tilde{Y}_{\square}}^{\mathrm{tp}} \subseteq \underline{\Pi}_{\tilde{X}_{\square}}^{\mathrm{tp}} \subseteq \underline{\Pi}_{X_{\square}}^{\mathrm{tp}}$, as desired. The profinite case is proven similarly [or may be derived from the tempered case via [Mzk14], Theorem 6.6].

Next, let us suppose that

$$
\sqrt{-1} \in K
$$

- where we note that " $\sqrt{-1}$ " determines a 4-torsion point $\tau$ of [the underlying elliptic curve of] $\dot{X}$ whose restriction to the special fiber lies in the interior of [i.e., avoids the nodes of] the unique irreducible component of the special fiber; the 4 -torsion point " $\tau$ " " determined by " $-\sqrt{-1}$ " admits a similar description. Let

$$
\ddot{\eta}^{\Theta} \in H^{1}\left(\underline{\Pi}_{\ddot{Y}}^{\mathrm{tp}}, \underline{\Delta}_{\Theta}\right)
$$

be a class as in Proposition 1.3; write $\ddot{\eta}^{\Theta,} \underline{\mathbb{Z}}$ for the $\underline{\Pi}_{\dot{X}}^{\mathrm{tp}} / \underline{\Pi}_{\ddot{Y}}^{\mathrm{tp}} \cong \underline{\mathbb{Z}}$-orbit of $\ddot{\eta}^{\Theta}$.

Definition 1.9. Suppose that $\sqrt{-1} \in K$.

(i) We shall refer to either of the following two sets of values [cf. Proposition 1.4, (iii)] of $\ddot{\eta}^{\Theta, \underline{\mathbb{Z}}}$

$$
\left.\ddot{\eta}^{\Theta, \underline{\mathbb{Z}}}\right|_{\tau},\left.\quad \ddot{\eta}^{\Theta, \underline{\mathbb{Z}}}\right|_{\tau^{-1}} \subseteq K^{\times}
$$

as a standard set of values of $\ddot{\eta}^{\Theta, \underline{Z}}$.

(ii) If $\ddot{\eta}^{\Theta}, \underline{\mathbb{Z}}$ satisfies the property that the unique value $\in \mathcal{O}_{K}^{\times}[\mathrm{cf}$. the value at $\sqrt{-1}$ of the series representation of $\ddot{\Theta}$ given in Proposition 1.4; Proposition 1.4, (ii)] of maximal order [i.e., relative to the valuation on $K$ ] of some standard set of values of $\ddot{\eta}^{\Theta,} \underline{\mathbb{Z}}$ is equal to \pm 1 , then we shall say that $\ddot{\eta}^{\Theta, \underline{\mathbb{Z}}}$ is of standard type.

Remark 1.9.1. Observe that it is immediate from the definitions that any inner automorphism of $\underline{\Pi}_{\dot{C}}^{\mathrm{tp}}$ arising from $\underline{\Pi}_{\dot{X}}^{\mathrm{tp}}$ acts trivially on $\ddot{\eta}^{\Theta}, \underline{\mathbb{Z}}$, and that the automorphisms $\epsilon_{\mu}, \epsilon_{ \pm}$map $\ddot{\eta}^{\Theta, \underline{\mathbb{Z}}} \mapsto-\ddot{\eta}^{\Theta,} \underline{\mathbb{Z}}$ [cf. Proposition 1.4, (ii)]. In

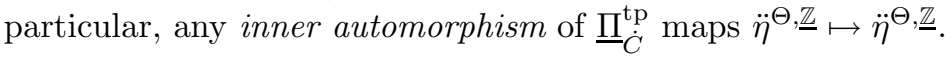

The point of view of Remark 1.6.1 motivates the following result:

Theorem 1.10 (Constant Multiple Rigidity of the Étale Theta Function). For $\square=\alpha, \beta$, let $\dot{C}_{\square}^{\log }$ be a smooth log curve of type $\left(1, \boldsymbol{\mu}_{2}\right)_{ \pm}$over a finite extension $K_{\square}$ of $\mathbb{Q}_{p}$ that contains a square root of -1 . Let

$$
\gamma: \underline{\Pi}_{\dot{C}_{\alpha}}^{\mathrm{tp}} \stackrel{\sim}{\rightarrow} \underline{\Pi}_{\dot{C}_{\beta}}^{\mathrm{tp}}
$$


be an isomorphism of topological groups. Suppose that the isomorphism $\underline{\Pi}_{X_{\alpha}}^{\mathrm{tp}} \stackrel{\sim}{\rightarrow}$ $\underline{\Pi}_{X_{\beta}}^{\mathrm{tp}}$ induced by $\gamma\left[c f\right.$. Proposition 1.8] maps $\ddot{\eta}_{\alpha}^{\Theta, \underline{\mathbb{Z}}} \mapsto \ddot{\eta}_{\beta}^{\Theta, \underline{\mathbb{Z}}}[c f$. Theorem 1.6, (iii)]. Then:

(i) The isomorphism $\gamma$ preserves the property that $\ddot{\eta}_{\square}^{\Theta, \underline{Z}}$ be of standard type, a property that determines this collection of classes up to multiplication by \pm 1 .

(ii) The isomorphism

$$
K_{\alpha}^{\times} \stackrel{\sim}{\rightarrow} K_{\beta}^{\times}
$$

- where we regard $K_{\square}^{\times} \subseteq\left(K_{\square}^{\times}\right)^{\wedge}$ as a subset of $\left(K_{\square}^{\times}\right)^{\wedge} \cong H^{1}\left(G_{K_{\square}},\left(\underline{\Delta}_{\Theta}\right) \square\right) \subseteq$ $H^{1}\left(\underline{\Pi}_{\dot{C}_{\square}}^{\mathrm{tp}},\left(\underline{\Delta}_{\Theta}\right)_{\square}\right)-$ induced by $[$ an arbitrary] $\gamma$ preserves the standard sets of values of $\ddot{\eta}_{\square}^{\Theta, \underline{\mathbb{Z}}}$.

(iii) Suppose that $\ddot{\eta}_{\square}^{\Theta, \mathbb{Z}}$ is of standard type, and that the residue characteristic of $K_{\square}$ is odd. Then $\ddot{\eta}_{\square}^{\Theta, \underline{Z}}$ determines a $\{ \pm 1\}$-structure [cf. [Mzk13], Corollary 4.12; Remark 1.10.1, (ii), below] on the $\left(K_{\square}^{\times}\right)^{\wedge}$-torsor at the unique cusp of $\dot{C}_{\square}^{\log }$ that is compatible with the canonical integral structure and, moreover, preserved by [arbitrary] $\gamma$.

Proof. First, we observe that assertions (i), (iii) follow formally from assertion (ii) [cf. also the series representation of Proposition 1.4]. Now we verify assertion (ii), as follows. By applying [Mzk14], Theorem 6.8, (iii), together with the fact that $\gamma$ induces isomorphisms between the dual graphs [cf. Theorem 1.6, (i); [Mzk2], Lemma 2.3] of the special fibers of the various corresponding coverings of $C_{\alpha}, C_{\beta}$ that appear in the proof of [Mzk14], Theorem 6.8, (iii), it follows that $\gamma$ maps [the decomposition group of points of $\ddot{Y}_{\alpha}$ lying over] $\tau$ to [the decomposition group of points of $\ddot{Y}_{\beta}$ lying over] $\tau^{ \pm 1}$. Now assertion (ii) follows immediately.

Remark 1.10.1.

(i) The " \pm -indeterminacy" of Theorem 1.10, (i), (iii), is reminiscent of, but stronger than, the indeterminacy up to multiplication by a 12-th root of unity of [Mzk13], Corollary 4.12. Also, we note that from the point of view of the technique of the proof of loc. cit., applied in the present context of "Tate curves", it is the fact that there is a "special 2-torsion point", i.e., the 2-torsion point whose image in the special fiber lies in the same irreducible component as the origin, that allows one to reduce the " $12=2 \cdot(3$ !)" of loc. cit. to "2".

(ii) We take this opportunity to remark that in [Mzk13], Corollary 4.12, the author omitted the hypothesis that " $K$ contain a primitive 12 -th root of unity". The author apologizes for this omission. 
Remark 1.10.2. One observation that one might make is that since Theorem 1.10 depends on the theory of [Mzk13], §2 [i.e., in the "tempered version" of this theory given in [Mzk14], §6], one natural approach to further strengthening Theorem 1.10 is to consider applying the "absolute -adic version of the Grothendieck Conjecture" of [Mzk16], Corollary 2.3, which may be regarded as a "strengthening" of the theory of [Mzk13], §2. One problem here, however, is that unlike the portion of the theory of [Mzk13], §2, that concerns [non-cuspidal] torsion points of once-punctured elliptic curves [i.e., [Mzk13], Corollary 2.6], the "absolute $p$-adic version of the Grothendieck Conjecture" of [Mzk16], Corollary 2.3 , only holds for elliptic curves which are defined over number fields. Moreover, even if, in the future, this hypothesis should be eliminated, the [somewhat weaker] theory of [Mzk13], §2, follows "formally" [cf. [Mzk13], Remark 2.8.1] from certain "general nonsense"-type arguments that hold over any base over which the relative isomorphism version of the Grothendieck Conjecture [i.e., the isomorphism portion of [Mzk11], Theorem A], together with the absolute preservation of cuspidal decomposition groups [cf. [Mzk13], Theorem 1.3, (iii)], holds. In particular, by restricting our attention to consequences of this "general nonsense" in the style of [Mzk13], 2 , one may hope to generalize the results discussed in the present $\S 1$ to much more general bases [such as, for instance, $\mathbb{Z}_{p}[[q]]\left[q^{-1}\right] \otimes \mathbb{Q}_{p}$, where $q$ is an indeterminate intended to suggest the " $q$-parameter of a Tate curve"], or, for instance, to the case of "pro- $\Sigma$ versions of the tempered fundamental group" [i.e., where $\Sigma$ is a set of primes containing $p$ which is not necessarily the set of all prime numbers] — situations in which it is by no means clear [at least at the time of writing] whether or not it is possible to prove an "absolute version of the Grothendieck Conjecture".

Remark 1.10.3.

(i) Note that the étale theta function arises as a cohomology class of a certain subgroup of the "theta quotient"

$$
\underline{\Pi}_{X}^{\mathrm{tp}} \rightarrow\left(\underline{\Pi}_{X}^{\mathrm{tp}}\right)^{\Theta}
$$

[cf. Propositions 1.3, 1.5]. On the other hand, the very strong rigidity property of Theorem 1.10, (i), clearly — as one may see, for instance, by considering automorphisms of the topological group $\left(\underline{\Pi}_{X}^{\mathrm{tp}}\right)^{\Theta}$ - fails to hold if, for instance, one replaces " $\underline{\Pi}_{C_{\square}}^{\mathrm{tp}}$ " by the corresponding "theta quotient" $\left(\underline{\Pi}_{C_{\square}}^{\mathrm{tp}}\right)^{\Theta}$. Thus, even though at first glance, the theory of the étale theta function may appear only to involve the theta quotient $\left(\underline{\Pi}_{X}^{\mathrm{tp}}\right)^{\Theta}$, in fact, the full tempered fundamental group $\underline{\Pi}_{X}^{\mathrm{tp}}$ plays an essential role in the theory of rigidity properties of the étale theta function. 
(ii) Relative to the eventual goal of applying the theory of the present paper to Hodge-Arakelov theory [cf. the Introduction] — which concerns restricting the theta function and its derivatives to torsion points - the necessity of working with the full tempered fundamental group may be motivated geometrically as follows. The hyperbolic orbicurves involved may be thought of, up to isogeny [cf. $§ 0]$, as the hyperbolic curves obtained by removing the l-torsion points [for some integer $l \geq 1$ ] from an elliptic curve [cf. especially the theory of $\S 2$ below]. At the level of topological surfaces, the complement $S_{\text {ell } \backslash \text { tors }}$ of the $l$-torsion points in an elliptic curve $E$ may be thought of as the result

$$
S_{\text {ell } \backslash \text { tors }}=S_{\text {ell } \backslash \text { disc }} \bigcup S_{\text {disc } \backslash \text { tors }}
$$

of gluing the complement $S_{\text {ell } \backslash \text { disc }}$ of a single disc in an elliptic curve to the complement $S_{\text {disc } \backslash \text { tors }}$ in a disc of a finite collection of points [i.e., where the gluing is along the circle which forms the boundary of the discs involved]. The topological fundamental group of $S_{\text {ell } \backslash \text { tors }}$ may then be thought of as the amalgamated sum of the fundamental groups of $S_{\text {ell } \backslash \text { disc }}, S_{\text {disc } \backslash \text { tors }}$. Now the fundamental group of $S_{\text {ell } \backslash \text { disc }}$ is a free group on two generators, which may be thought of as a basis of the abelian fundamental group of the original elliptic curve $E$. This portion of the fundamental group of $S_{\text {ell } \backslash \text { tors }}$ may be thought of as corresponding to the "Heisenberg group", or theta-group - i.e., the theta quotient discussed in (i) — which plays a fundamental role in the theory of theta functions, hence in the scheme-theoretic Hodge-Arakelov theory reviewed in [Mzk4] [cf. especially the discussion of [Mzk4], §1.3.5]. On the other hand, $S_{\text {disc } \backslash \text { tors }}$ is homotopically equivalent to a bouquet of circles, where the circles correspond naturally to the $l$-torsion points of $E$. Thus, from the point of view of algebraic topology, the $S_{\text {disc } \backslash \text { tors }}$ portion of $S_{\text {ell } \backslash \text { tors }}$ may be thought of as the suspension of the discrete set of $l$-torsion points [together with some additional "basepoint"]. On the other hand, from the point of view of arithmetic geometry, the circle may be thought of as a sort of "Tate motive" and the "arithmetic suspension" constituted by the $S_{\text {disc } \backslash \text { tors }}$ portion of $S_{\text {ell } \backslash \text { tors }}$ as a sort of "anabelian Tate twist" of the l-torsion points of $E$. Moreover, from the point of view of Hodge-Arakelov theory, the universal covering of the bouquet constituted by $S_{\text {disc } \backslash \text { tors }}$ may be thought of as the result of "filling in the discrete set of l-torsion points" by joining these points via continuous line segments cf. the original point of view of Hodge-Arakelov theory [discussed in [Mzk4], $\S 1.3 .4]$ to the effect that the set of torsion points is to be regarded as a "high resolution approximation" of the underlying real analytic manifold of $E$. Put another way, this sort of "continuous version of the $l$-torsion points" may be thought of as a sort of "cyclotomic blurring" of the l-torsion points - i.e., a sort 
of space of infinitesimal deformations of the $l$-torsion points that allows one to consider derivatives [e.g., of the theta function] at the $l$-torsion points. Thus, in summary, relative to these geometric considerations, the topological surface $S_{\text {ell } \backslash \text { tors }}=S_{\text {ell } \backslash \text { disc }} \cup S_{\text {disc } \backslash \text { tors }}$, hence also its group-theoretic counterpart [i.e., the full fundamental group under consideration] may be thought of as being precisely a geometric realization of the content

$$
\text { (theta functions and their derivatives) }\left.\right|_{l \text {-torsion points }}
$$

[i.e., where "theta functions" correspond, via theta-groups, to $S_{\text {ell } \backslash \text { disc }}$, and "derivatives at $l$-torsion points" correspond to $\left.S_{\text {disc } \backslash \text { tors }}\right]$ of Hodge-Arakelov theory.

\section{Remark 1.10.4.}

(i) As observed in Proposition 1.4, (iii), by restricting the étale theta function to various [e.g., torsion!] points, one obtains a Kummer-theoretic approach to considering the theta function as a function on points. In the context of the theory of the present $\S 1$ [and indeed of the present paper], in which one does not assume [cf. Remark 1.10.2] that the multiplicative groups associated to the base fields involved that appear in the absolute Galois groups of these fields e.g., the " $\left(L^{\times}\right)^{\wedge}$ " that appears in Proposition 1.4, (iii) — are equipped with additive structures [i.e., arising from the addition operation on the field], the functions that may be obtained in this way are very special. Indeed, if one may avail oneself of both the additive and multiplicative structures - i.e., the ring structures - of the fields involved, then it is not difficult to give various "group-theoretic algorithms" for constructing all sorts of such "functions". On the other hand:

If one may only avail oneself of the multiplicative structure, then it is difficult to construct such functions, except via considering directly the functions obtained by restricting Kummer classes of meromorphic functions.

[Of course, the multiplicative structure allows one to construct $N$-th powers, for $N \geq 1$ an integer, of the values obtained by restricting Kummer classes, but such $N$-th powers are simply the values obtained by restricting the Kummer classes obtained by multiplying the original Kummer classes by $N$.] Finally, we recall that the theta function satisfies the unusual property, among meromorphic functions on tempered coverings of pointed stable curves, of having a divisor of poles that is contained in the special fiber and a divisor zeroes that 
does not contain any irreducible components of the special fiber [cf. Proposition $1.4,(\mathrm{i})]$.

(ii) Even if one allows oneself to consider "Kummer classes"

$$
\kappa \in H^{1}(\Pi, \widehat{\mathbb{Z}}(1))
$$

for $\Pi$ an arbitrary topological group that surjects onto the absolute Galois group $G_{K}$ of a finite extension $K$ of $\mathbb{Q}_{p}$, it is not difficult to see that it is a highly nontrivial operation to construct functions on the set of [conjugacy classes of] sections of $\Pi \rightarrow G_{K}$. Indeed, if $\kappa$ is to give rise to a nontrivial function, then it is natural to assume that it must induce an isomorphism of some isomorph [cf. $\S 0$ ] of $\widehat{\mathbb{Z}}(1)$ inside $\Pi$ onto $\widehat{\mathbb{Z}}(1)$ [cf. the role of " $\underline{\Delta}_{\Theta}$ " in Proposition 1.3 in the case of the étale theta function]. On the other hand, if, for instance, $\Pi=\widehat{\mathbb{Z}}(1) \rtimes G_{K}$, then [cf. the discussion of the "theta quotient" in Remark 1.10 .3 , (i)] it is easy to see that the resulting $\kappa$ fails to satisfy the analogue of the rigidity property of Theorem 1.10, (i). Thus, just as in the discussion of Remark 1.10.3, one is ultimately led naturally to consider the case where $\Pi$ is some sort of [e.g., tempered or profinite] arithmetic group of a hyperbolic orbicurve, in which case various strong anabelian rigidity properties are known. Moreover, it is difficult to see how to develop the theory of $\S 2$ below - which makes essential use, in so many ways, of the theory and structure of theta-groups [i.e., the "theta quotients" of Remark 1.10.3, (i)] — for hyperbolic orbicurves that are not isogenous to once-punctured elliptic curves.

This state of affairs again serves to highlight the fact that the function [on, say, torsion points] determined by the étale theta function should be regarded as a very special and unusual object.

\section{§2. The Theory of Theta Environments}

In this $\S$, we begin by discussing various "general nonsense" complements [cf. Corollaries 2.8, 2.9] to the theory of étale theta functions of $\S 1$ involving coverings [cf. the discussion of the "Lagrangian approach to Hodge-Arakelov theory" in the Introduction]. This discussion leads naturally to the theory of the cyclotomic envelope [cf. Definition 2.10] and the associated mono- and bi-theta environments [cf. Definition 2.13], whose tempered anabelian rigidity properties [cf. Corollaries 2.18, 2.19] we shall use in $\S 5$, below, to relate the theory of the present $\S 2$ to the theory of tempered Frobenioids to be discussed in $\S 3, \S 4$, below. 
Let $X^{\log }$ be a smooth log curve of type $(1,1)$ over a field $K$ of characteristic zero. For simplicity, we assume that the hyperbolic curve determined by $X^{\log }$ is not $K$-arithmetic [i.e., admits a $K$-core - cf. [Mzk3], Remark 2.1.1]. As in $\S 1$, we shall denote the (profinite) étale fundamental group of $X^{\log }$ by $\underline{\Pi}_{X}$. Thus, we have a natural exact sequence:

$$
1 \rightarrow \underline{\Delta}_{X} \rightarrow \underline{\Pi}_{X} \rightarrow G_{K} \rightarrow 1
$$

— where $G_{K} \stackrel{\text { def }}{=} \operatorname{Gal}(\bar{K} / K) ; \underline{\Delta}_{X}$ is defined so as to make the sequence exact. Since $\underline{\Delta}_{X}$ is a profinite free group on 2 generators, the quotient

$$
\underline{\Delta}_{X}^{\Theta} \stackrel{\text { def }}{=} \underline{\Delta}_{X} /\left[\underline{\Delta}_{X},\left[\underline{\Delta}_{X}, \underline{\Delta}_{X}\right]\right]
$$

fits into a natural exact sequence

$$
1 \rightarrow \underline{\Delta}_{\Theta} \rightarrow \underline{\Delta}_{X}^{\Theta} \rightarrow \underline{\Delta}_{X}^{\mathrm{ell}} \rightarrow 1
$$

— where $\underline{\Delta}_{X}^{\text {ell }} \stackrel{\text { def }}{=} \underline{\Delta}_{X}^{\mathrm{ab}}=\underline{\Delta}_{X} /\left[\underline{\Delta}_{X}, \underline{\Delta}_{X}\right]$; we write $\underline{\Delta}_{\Theta}$ for the image of $\wedge^{2} \underline{\Delta}_{X}^{\text {ell }}$ in $\underline{\Delta}_{X}^{\Theta}$. Also, we shall write $\underline{\Pi}_{X} \rightarrow \underline{\Pi}_{X}^{\Theta}$ for the quotient whose kernel is the kernel of the quotient $\underline{\Delta}_{X} \rightarrow \underline{\Delta}_{X}^{\Theta}$.

Now let $l \geq 1$ be an integer. One verifies easily by considering the wellknown structure of $\Delta_{X}^{\Theta}$ that the subgroup of $\underline{\Delta}_{X}^{\ominus}$ generated by l-th powers of elements of $\Delta_{X}^{\ominus}$ is normal. We shall write $\underline{\Delta}_{X}^{\ominus} \rightarrow \bar{\Delta}_{X}$ for the quotient of $\underline{\Delta}_{X}^{\ominus}$ by this normal subgroup. Thus, the above exact sequence for $\underline{\Delta}_{X}^{\Theta}$ determines a quotient exact sequence

$$
1 \rightarrow \bar{\Delta}_{\Theta} \rightarrow \bar{\Delta}_{X} \rightarrow \bar{\Delta}_{X}^{\mathrm{ell}} \rightarrow 1
$$

— where $\bar{\Delta}_{\Theta} \cong(\mathbb{Z} / l \mathbb{Z})(1) ; \bar{\Delta}_{X}^{\text {ell }}$ is a free $(\mathbb{Z} / l \mathbb{Z})$-module of rank 2. Also, we shall write $\underline{\Pi}_{X} \rightarrow \bar{\Pi}_{X}$ for the quotient whose kernel is the kernel of the quotient $\underline{\Delta}_{X} \rightarrow \bar{\Delta}_{X}$ and $\bar{\Pi}_{X}^{\text {ell }} \stackrel{\text { def }}{=} \bar{\Pi}_{X} / \bar{\Delta}_{\Theta}$.

Let us write $x$ for the unique cusp of $X^{\log }$. Then there is a natural injective [outer] homomorphism

$$
D_{x} \hookrightarrow \underline{\Pi}_{X}^{\Theta}
$$

- where $D_{x} \subseteq \underline{\Pi}_{X}$ is the decomposition group associated to $x$ - which maps the inertia group $I_{x} \subseteq D_{x}$ isomorphically onto $\underline{\Delta}_{\Theta}$. Thus, we have exact sequences

$$
1 \rightarrow \bar{\Delta}_{X} \rightarrow \bar{\Pi}_{X} \rightarrow G_{K} \rightarrow 1 ; \quad 1 \rightarrow \bar{\Delta}_{\Theta} \rightarrow \bar{D}_{x} \rightarrow G_{K} \rightarrow 1
$$

— where we write $\bar{D}_{x} \subseteq \bar{\Pi}_{X}$ for the image of $D_{x}$ in $\bar{\Pi}_{X}$. 
Next, let $\bar{\Pi}_{X}^{\text {ell }} \rightarrow Q$ be a quotient onto a free $(\mathbb{Z} / l \mathbb{Z})$-module $Q$ of rank 1 such that the restricted map $\bar{\Delta}_{X}^{\text {ell }} \rightarrow Q$ is still surjective, but the restricted map $D_{x} \rightarrow Q$ is trivial. Denote the corresponding covering by $\underline{X}^{\log } \rightarrow X^{\log }$; write $\bar{\Pi}_{\underline{X}} \subseteq \bar{\Pi}_{X}, \bar{\Delta}_{\underline{X}} \subseteq \bar{\Delta}_{X}, \bar{\Delta}_{X}^{\text {ell }} \subseteq \bar{\Delta}_{X}^{\text {ell }}$ for the corresponding open subgroups. Observe that our assumption that the restricted map $D_{x} \rightarrow Q$ is trivial implies that every cusp of $\underline{X}^{\log }$ is $K$-rational. Let us write $\iota$ (respectively, $\underline{\iota}$ ) for the automorphism of $X^{\log }$ (respectively, $\underline{X}^{\log }$ ) determined by "multiplication by -1 " on the underlying elliptic curve relative to choosing the unique cusp of $X^{\log }$ (respectively, relative to some choice of a cusp of $\underline{X}^{\log }$ ) as the origin. Thus, if we denote the stack-theoretic quotient of $X^{\log }$ (respectively, $\underline{X}^{\log }$ ) by the action of $\iota$ (respectively, $\underline{\iota}$ ) by $C^{\log }$ (respectively, $\left.\underline{C}^{\log }\right)$, then we have a cartesian commutative diagram:

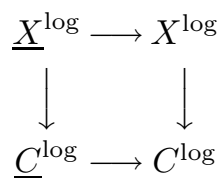

We shall write $\underline{\Pi}_{C}, \underline{\Pi}_{C}$ for the respective (profinite) étale fundamental groups of $\underline{C}^{\log }, C^{\log }$. Thus, we obtain subgroups $\underline{\Delta}_{C} \subseteq_{\underline{\Pi}}, \underline{\Delta}_{C} \subseteq \underline{\Pi}_{C}$ [i.e., the kernels of the natural surjections to $\left.G_{K}\right]$; moreover, by forming the quotient by the kernels of the quotients $\underline{\Pi}_{X} \rightarrow \bar{\Pi}_{\underline{X}}, \underline{\Pi}_{X} \rightarrow \bar{\Pi}_{X}$, we obtain quotients $\underline{\Pi}_{C} \rightarrow$ $\bar{\Pi}_{\underline{C}}, \underline{\Pi}_{C} \rightarrow \bar{\Pi}_{C}, \underline{\Delta}_{\underline{C}} \rightarrow \bar{\Delta}_{\underline{C}}, \underline{\Delta}_{C} \rightarrow \bar{\Delta}_{C}$. Similarly, the quotient $\bar{\Delta}_{X} \rightarrow \bar{\Delta}_{X}^{\text {ell }}$ determines a quotient $\bar{\Delta}_{C} \rightarrow \bar{\Delta}_{C}^{\text {ell }}$. Let $\epsilon_{\underline{\underline{L}}} \in \bar{\Delta}_{\underline{C}}$ be an element that lifts the nontrivial element of $\operatorname{Gal}(\underline{X} / \underline{C}) \cong \mathbb{Z} / 2 \mathbb{Z}$.

Definition 2.1. We shall refer to a smooth log orbicurve over $K$ that arises, up to isomorphism, as the smooth log orbicurve $\underline{X}^{\log }$ (respectively, $\underline{C}^{\log }$ ) constructed above for some choice of $\bar{\Pi}_{X}^{\text {ell }} \rightarrow Q$ as being of type (1,l-tors) (respectively, $\left.(1, l \text {-tors })_{ \pm}\right)$. We shall also apply this terminology to the associated hyperbolic orbicurves.

Remark 2.1.1. Note that although $\underline{X}^{\log } \rightarrow X^{\log }$ is [by construction] $G a$ lois, with $\operatorname{Gal}(\underline{X} / X) \cong Q$, the covering $\underline{C}^{\log } \rightarrow C^{\log }$ fails to be Galois in general. More precisely, no nontrivial automorphism $\in \operatorname{Gal}(\underline{X} / X)$ of, say, odd order descends to an automorphism of $\underline{C}^{\log }$ over $C^{\log }$. Indeed, this follows from the fact that $\underline{\iota}$ acts on $Q$ by multiplication by -1 .

Proposition 2.2 (The Inversion Automorphism). Suppose that $l$ is odd. Then: 
(i) The conjugation action of $\epsilon_{\underline{\underline{L}}}$ on the rank two $(\mathbb{Z} / l \mathbb{Z})$-module $\bar{\Delta}_{\underline{X}}$ determines a direct product decomposition

$$
\bar{\Delta}_{\underline{X}} \cong \bar{\Delta}_{\underline{X}}^{\mathrm{ell}} \times \bar{\Delta}_{\Theta}
$$

into eigenspaces, with eigenvalues -1 and 1 , respectively, that is compatible with the conjugation action of $\bar{\Pi}_{\underline{X}}$. Denote by

$$
s_{\iota}: \bar{\Delta}_{\underline{X}}^{\mathrm{ell}} \rightarrow \bar{\Delta}_{\underline{X}}
$$

the resulting splitting of the natural surjection $\bar{\Delta}_{\underline{X}} \rightarrow \bar{\Delta}_{\underline{X}}^{\text {ell }}$.

(ii) In the notation of $(i)$, the normal subgroup $\operatorname{Im}\left(s_{\iota}\right) \subseteq \bar{\Pi}_{\underline{X}}$ induces an isomorphism

$$
\bar{D}_{x} \stackrel{\sim}{\rightarrow} \bar{\Pi}_{\underline{X}} / \operatorname{Im}\left(s_{\iota}\right)
$$

over $G_{K}$. In particular, any section of the $H^{1}\left(G_{K}, \bar{\Delta}_{\Theta}\right) \cong K^{\times} /\left(K^{\times}\right)^{l}$-torsor of splittings of $\bar{D}_{x} \rightarrow G_{K}$ determines a covering

$$
\underline{\underline{X}}^{\log } \rightarrow \underline{X}^{\log }
$$

whose corresponding open subgroup we denote by $\bar{\Pi}_{\underline{\underline{X}}} \subseteq \bar{\Pi}_{\underline{X}}$. Here, the "geometric portion" $\bar{\Delta}_{\underline{\underline{X}}}$ of $\bar{\Pi}_{\underline{\underline{X}}}$ maps isomorphically onto $\bar{\Delta}_{\underline{X}}^{\text {ell }}$ [hence is a cyclic group of order $l]$, i.e., we have ${\overline{\Delta_{X}}}_{\underline{\underline{X}}}=\operatorname{Im}\left(s_{\iota}\right), \bar{\Delta}_{\underline{X}}={\overline{\Delta_{\underline{X}}}}_{\underline{\underline{X}}} \cdot \bar{\Delta}_{\Theta}$. Finally, the image of $\epsilon_{\underline{\underline{L}}}$ in $\bar{\Delta}_{C} / \bar{\Delta}_{\underline{X}}$ may be characterized as the unique coset of $\bar{\Delta}_{C} / \bar{\Delta}_{\underline{X}}$ that lifts the nontrivial element of $\operatorname{Gal}(X / C)=\bar{\Delta}_{C} / \bar{\Delta}_{X}$ and normalizes the subgroup $\bar{\Delta}_{\underline{\underline{X}}} \subseteq \bar{\Delta}_{C}$.

(iii) There exists a unique coset $\in \bar{\Delta}_{\underline{C}} / \bar{\Delta}_{\underline{X}}$ such that $\epsilon_{\underline{\underline{L}}}$ has order 2 if and only if it belongs to this coset. If we choose $\epsilon_{\underline{\underline{L}}}$ to have order 2 , then the open subgroup generated by $\bar{\Pi}_{\underline{\underline{X}}}$ and $\epsilon_{\underline{L}}$ in $\bar{\Pi}_{\underline{C}}$ [or, alternatively, the open subgroup generated by $G_{K} \cong \bar{\Pi}_{\underline{\underline{X}}} / \overline{\bar{\Delta}}_{\underline{\underline{X}}}$ and $\bar{\epsilon}_{\underline{\underline{L}}}$ in ${\overline{\Pi_{\underline{C}}}}_{\underline{\bar{\Delta}_{\underline{X}}}}$ determines a double covering $\underline{\underline{X}}^{\log } \rightarrow \underline{\underline{C}}^{\log }$ which fits into a cartesian commutative diagram

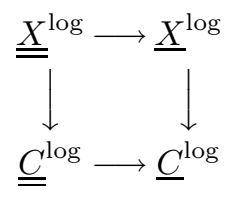

- where $\underline{\underline{X}}^{\log }$ is as in (ii).

Proof. Assertions (i) and (ii) are immediate from the definitions. To verify assertion (iii), we observe that $\bar{D}_{x} \cong \bar{\Pi}_{\underline{X}} / \bar{\Delta}_{\underline{\underline{X}}}$ is of index 2 in $\bar{\Pi}_{\underline{C}} / \bar{\Delta}_{\underline{\underline{X}}}$. 
Thus, $\epsilon_{\underline{\underline{L}}}$ normalizes $\bar{D}_{x} \cong \bar{\Pi}_{\underline{X}} / \bar{\Delta}_{\underline{X}}$. Since, moreover, $l$ is odd [so $H^{1}\left(G_{K}, \bar{\Delta}_{\Theta}\right)$, $\bar{\Delta}_{\Theta} / H^{0}\left(G_{K}, \bar{\Delta}_{\Theta}\right)$ have no elements of order 2], and conjugation by $\epsilon_{\underline{L}}$ induces the identity on $\bar{\Delta}_{\Theta}$ and $G_{K}$, it follows that $\epsilon_{\underline{\underline{L}}}$ centralizes $\bar{D}_{x} \cong \bar{\Pi}_{\underline{X}} / \bar{\Delta}_{\underline{\underline{X}}}$, hence [a fortiori] $G_{K} \cong \bar{\Pi}_{\underline{\underline{X}}} / \bar{\Delta}_{\underline{\underline{X}}}$. Now assertion (iii) follows immediately.

Remark 2.2.1. We shall not discuss the case of even $l$ in detail here. Nevertheless, we pause briefly to observe that if $l=2$, then [since $\bar{\Delta}_{\Theta}$ lies in the center of $\left.\bar{\Delta}_{X}\right]$ the automorphism $\epsilon_{ \pm} \in \operatorname{Gal}(\ddot{X} / C) \cong \bar{\Delta}_{C}^{\text {ell }}$ of $\S 1$ acts naturally on the exact sequence $1 \rightarrow \bar{\Delta}_{\Theta} \rightarrow \bar{\Delta}_{X} \rightarrow \bar{\Delta}_{X}^{\text {ell }} \rightarrow 1$. Since this action is clearly trivial on $\bar{\Delta}_{\Theta}, \bar{\Delta}_{X}^{\text {ell }}$, one verifies immediately that this action determines a homomorphism $\bar{\Delta}_{X}^{\text {ell }} \rightarrow \bar{\Delta}_{\Theta}$, i.e., in effect, a 2-torsion point [so long as the homomorphism is nontrivial] of the elliptic curve underlying $X^{\log }$. Thus, by considering the case where $K$ is the field of moduli of this elliptic curve [so that $G_{K}$ permutes the 2-torsion points transitively], we conclude that this homomorphism must be trivial, i.e., that every element of $\bar{\Delta}_{X}^{\text {ell }}$ admits an $\epsilon_{ \pm}$-invariant lifting to $\bar{\Delta}_{X}$.

Definition 2.3. We shall refer to a smooth log orbicurve over $K$ that arises, up to isomorphism, as the smooth log orbicurve $\underline{\underline{X}}^{\log }$ (respectively, $\underline{\underline{C}}^{\log }$ ) constructed in Proposition 2.2 above as being of type $\left(1, l\right.$-tors ${ }^{\Theta}$ ) (respectively, $\left.\left(1, l \text {-tors }{ }^{\Theta}\right)_{ \pm}\right)$. We shall also apply this terminology to the associated hyperbolic orbicurves.

Remark 2.3.1. Thus, one may think of the "single underline" in the notation $\underline{X}^{\log }, \underline{C}^{\log }$ as denoting the result of "extracting a single copy of $\mathbb{Z} / l \mathbb{Z}$ ", and the "double underline" in the notation $\underline{\underline{X}}^{\log }, \underline{\underline{C}}^{\log }$ as denoting the result of "extracting two copies of $\mathbb{Z} / l \mathbb{Z}$ ".

Proposition 2.4 (Characteristic Nature of Coverings). $\quad$ For $\square=\alpha, \beta$, let $\underline{\underline{X}}_{\square}^{\log }$ be a smooth log curve of type $\left(1, l\right.$-tors $\left.{ }^{\Theta}\right)$ over a finite extension $K_{\square}$ of $\mathbb{Q}_{p}$, where l is odd; write $\underline{\underline{C}}_{\square}^{\log }, \underline{X}_{\square}^{\log }, \underline{C}_{\square}^{\log }, X_{\square}^{\log }, C_{\square}^{\log }$ for the related smooth log orbicurves [as in the above discussion]. Assume further that $X_{\square}^{\log }$ has stable reduction over $\mathcal{O}_{K_{\square}}$, with singular and split special fiber. Then any isomorphism of topological groups

$$
\begin{aligned}
& \gamma: \underline{\Pi}_{\underline{\underline{X}}_{\alpha}}^{\mathrm{tp}} \stackrel{\sim}{\rightarrow} \underline{\Pi}_{\underline{\underline{X}}_{\beta}}^{\mathrm{tp}} \text { (respectively, } \gamma: \underline{\Pi}_{\underline{X}_{\alpha}}^{\mathrm{tp}} \stackrel{\sim}{\rightarrow} \underline{\Pi}_{\underline{X}_{\beta}}^{\mathrm{tp}} ; \\
& \left.\gamma: \underline{\Pi}_{\underline{\underline{C}}_{\alpha}}^{\mathrm{tp}} \stackrel{\sim}{\rightarrow} \underline{\Pi}_{\underline{\underline{\Pi}}_{\beta}}^{\mathrm{tp}} ; \gamma: \underline{\Pi}_{\underline{\Pi}_{\alpha}}^{\mathrm{tp}} \stackrel{\sim}{\rightarrow} \underline{\Pi}_{\underline{\Pi}_{\beta}}^{\mathrm{tp}}\right)
\end{aligned}
$$

induces isomorphisms compatible with the various natural maps between the respective " $\underline{\Pi}^{\text {tp }}$ 's" of $\underline{X}_{\square}^{\log }, X_{\square}^{\log }, \underline{\underline{C}}_{\square}^{\log }, \underline{C}_{\square}^{\log }, C_{\square}^{\log }, \ddot{Y}_{\square}^{\log }$ (respectively, $X_{\square}^{\log }$, 
$\left.C_{\square}^{\log }, \ddot{Y}_{\square}^{\log } ; \underline{C}_{\square}^{\log }, C_{\square}^{\log }, \underline{X}_{\square}^{\log }, \underline{X}_{\square}^{\log }, X_{\square}^{\log }, \ddot{Y}_{\square}^{\log } ; C_{\square}^{\log }, \underline{X}_{\square}^{\log }, X_{\square}^{\log }, \ddot{Y}_{\square}^{\log }\right)$ where $\square=\alpha, \beta$.

Proof. As in the proof of Proposition 1.8, it follows from our assumption that the hyperbolic curve determined by $X_{\square}^{\log }$ admits a $K_{\square \text {-core that } \gamma \text { induces }}$ an isomorphism $\underline{\Pi}_{C_{\alpha}}^{\mathrm{tp}} \stackrel{\sim}{\rightarrow} \underline{\Pi}_{C_{\beta}}^{\mathrm{tp}}$ [cf. [Mzk3], Theorem 2.4] which [cf. [Mzk2], Lemma 1.3.8] induces an isomorphism $\underline{\Delta}_{C_{\alpha}}^{\mathrm{tp}} \stackrel{\sim}{\rightarrow} \underline{\Delta}_{C_{\beta}}^{\mathrm{tp}}$; moreover, this last isomorphism induces [by considering open subgroups of index 2 whose profinite completions contain no torsion elements] an isomorphism $\underline{\Delta}_{X_{\alpha}}^{\mathrm{tp}} \stackrel{\sim}{\rightarrow} \underline{\Delta}_{X_{\beta}}^{\mathrm{tp}}$, hence also [by considering the conjugation action of $\underline{\Pi}_{C_{\square}}^{\mathrm{tp}}$ on an appropriate abelian quotient of $\underline{\Delta}_{X_{\square}}^{\mathrm{tp}}$ as in the proof of Proposition 1.8] an isomorphism $\underline{\Pi}_{X_{\alpha}}^{\mathrm{tp}} \stackrel{\sim}{\rightarrow} \underline{\Pi}_{X_{\beta}}^{\mathrm{tp}}$, which preserves the decomposition groups of cusps [cf. [Mzk14], Theorem 6.5, (iii)]. Also, by the definition of $\bar{\Delta}_{X_{\square}}$, the isomorphism $\underline{\Delta}_{X_{\alpha}}^{\text {tp }} \stackrel{\sim}{\rightarrow}{\stackrel{\Delta}{X_{\beta}}}_{\text {tp }}$ determines an isomorphism $\bar{\Delta}_{X_{\alpha}} \stackrel{\sim}{\rightarrow} \bar{\Delta}_{X_{\beta}}$. In light of these observations, the various assertions of Proposition 2.4 follow immediately from the definitions [cf. also Proposition 2.2; Theorem 1.6, (i); the proof of Proposition 1.8].

Now, we return to the discussion of $\S 1$. In particular, we assume that $K$ is a finite extension of $\mathbb{Q}_{p}$.

Definition 2.5. Suppose that $l$ and the residue characteristic of $K$ are $o d d$, and that $K=\ddot{K}$ [cf. Definition 1.7 and the preceding discussion].

(i) Suppose, in the situation of Definitions 2.1, 2.3, that the quotient $\bar{\Pi}_{X}^{\text {ell }} \rightarrow$ $Q$ factors through the natural quotient $\underline{\Pi}_{X} \rightarrow \widehat{\mathbb{Z}}$ determined by the quotient $\underline{\Pi}_{X}^{\mathrm{tp}} \rightarrow \mathbb{Z}$ discussed at the beginning of $\S 1$, and that the choice of a splitting of $\bar{D}_{x} \rightarrow G_{K}$ [cf. Proposition 2.2, (ii)] that determined the covering $\underline{X}^{\log } \rightarrow$ $\underline{X}^{\log }$ is compatible with the " $\{ \pm 1\}$-structure" of Theorem 1.10, (iii). Then we shall say that the orbicurve of type (1,l-tors) (respectively, $\left(1, l\right.$-tors $\left.{ }^{\Theta}\right)$; $\left.(1, l \text {-tors })_{ \pm} ;\left(1, l \text {-tors }{ }^{\Theta}\right)_{ \pm}\right)$under consideration is of type $(1, \mathbb{Z} / l \mathbb{Z})$ (respectively, $\left.\left(1,(\mathbb{Z} / l \mathbb{Z})^{\Theta}\right) ;(1, \mathbb{Z} / l \mathbb{Z})_{ \pm} ;\left(1,(\mathbb{Z} / l \mathbb{Z})^{\Theta}\right)_{ \pm}\right)$.

(ii) In the notation of the above discussion and the discussion at the end of $\S 1$, we shall refer to a smooth log orbicurve isomorphic to the smooth log orbicurve

$$
\underline{\dot{X}}^{\log } \text { (respectively, } \underline{\underline{X}}^{\log } ; \underline{\dot{C}}^{\log } ; \underline{\underline{C}}^{\log } \text { ) }
$$

obtained by taking the composite of the covering

$$
\underline{X}^{\log } \text { (respectively, } \underline{\underline{X}}^{\log } ; \underline{C}^{\log } ; \underline{\underline{C}}^{\log } \text { ) }
$$

of $C^{\text {log }}$ with the covering $\dot{C}^{\log } \rightarrow C^{\text {log }}$, as being of type $\left(1, \boldsymbol{\mu}_{2} \times \mathbb{Z} / l \mathbb{Z}\right.$ ) (respectively, $\left.\left(1, \boldsymbol{\mu}_{2} \times(\mathbb{Z} / l \mathbb{Z})^{\Theta}\right) ;\left(1, \boldsymbol{\mu}_{2} \times \mathbb{Z} / l \mathbb{Z}\right)_{ \pm} ;\left(1, \boldsymbol{\mu}_{2} \times(\mathbb{Z} / l \mathbb{Z})^{\Theta}\right)_{ \pm}\right)$. 
Remark 2.5.1. Thus, the irreducible components of the special fiber of $\underline{C}$, $\underline{\dot{C}}$ may be naturally identified with the elements of $(\mathbb{Z} / l \mathbb{Z}) /\{ \pm 1\}-$ cf. Corollary 2.9 below for more details.

Proposition 2.6 (Characteristic Nature of Coverings). $\quad$ For $\square=\alpha, \beta$, let us assume that we have smooth log orbicurves as in the above discussion, over a finite extension $K_{\square}$ of $\mathbb{Q}_{p}$. Then any isomorphism of topological groups

$$
\begin{aligned}
\gamma: \underline{\Pi}_{\dot{\dot{X}}_{\alpha}}^{\mathrm{tp}} \stackrel{\sim}{\rightarrow} \underline{\Pi}_{\underline{\dot{X}}^{\mathrm{x}}}^{\mathrm{tp}} \quad \text { (respectively, } \gamma: \underline{\Pi}_{\underline{\dot{X}}_{\alpha}}^{\mathrm{tp}} \stackrel{\sim}{\rightarrow} \underline{\Pi}_{\underline{\dot{X}}_{\beta}}^{\mathrm{tp}} ; \\
\left.\gamma: \underline{\Pi}_{\underline{\dot{C}}_{\alpha}}^{\mathrm{p}} \stackrel{\sim}{\rightarrow} \underline{\Pi}_{\underline{\dot{C}}_{\beta}}^{\mathrm{tp}} ; \gamma: \underline{\Pi}_{\underline{\dot{C}}_{\alpha}}^{\mathrm{tp}} \stackrel{\sim}{\rightarrow} \underline{\Pi}_{\underline{\dot{C}}_{\beta}}^{\mathrm{tp}}\right)
\end{aligned}
$$

induces isomorphisms compatible with the various natural maps between the respective " $\underline{\Pi}^{\mathrm{tp}}$ 's" of $\underline{\underline{X}}_{\square}^{\log }$ (respectively, $\underline{X}_{\square}^{\log } ; \underline{\underline{C}}_{\square}^{\log } ; \underline{C}_{\square}^{\log }$ ) and $\dot{C}_{\square}^{\log }$, where $\square=$ $\alpha, \beta$. A similar statement holds when " $\underline{\Pi}$ " $"$ is replaced by " $\underline{\Pi}$ ".

Proof. The proof is entirely similar to the proofs of Propositions 1.8, 2.4 .

Remark 2.6.1. Suppose, for simplicity, that $K$ contains a primitive $l$-th root of unity. Then we observe in passing that by applying the Propositions 2.4, 2.6 to "isomorphisms of fundamental groups arising from isomorphisms of the orbicurves in question" [cf. also Remark 2.1.1], one computes easily that the groups of $K$-linear automorphisms "Aut ${ }_{K}(-)$ " of the various smooth log orbicurves under consideration are given as follows:

$$
\begin{aligned}
\operatorname{Aut}_{K}\left(\underline{\underline{X}}^{\log }\right)=\boldsymbol{\mu}_{l} \times\{ \pm 1\} ; & \operatorname{Aut}_{K}\left(\underline{X}^{\log }\right)=\mathbb{Z} / l \mathbb{Z} \rtimes\{ \pm 1\} \\
\operatorname{Aut}_{K}\left(\underline{\underline{C}}^{\log }\right)=\boldsymbol{\mu}_{l} ; & \operatorname{Aut}_{K}\left(\underline{C}^{\log }\right)=\{1\}
\end{aligned}
$$

- where $\boldsymbol{\mu}_{l}$ denotes the group of $l$-th roots of unity in $K$, and the semi-direct product " $\rtimes$ " is with respect to the natural multiplicative action of \pm 1 on $\mathbb{Z} / l \mathbb{Z}$; the "Aut ${ }_{K}(-)$ 's" of the various "once-dotted versions" of these orbicurves [cf. Definition 2.5, (ii)] are given by taken the direct product of the "Aut ${ }_{K}(-)$ 's" listed above with $\operatorname{Gal}\left(\dot{C}^{\log } / C^{\log }\right) \cong\{ \pm 1\}$.

Next, we consider étale theta functions. First, let us observe that the covering $\ddot{Y} \log \rightarrow C^{\log }$ factors naturally through $\underline{\dot{X}}^{\log }$. Thus, the class [which is only well-defined up to a $\mathcal{O}_{K}^{\times}$-multiple]

$$
\ddot{\eta}^{\Theta} \in H^{1}\left(\underline{\Pi}_{\ddot{Y}}^{\mathrm{tp}}, \underline{\Delta}_{\Theta}\right)
$$


of $\S 1$ - as well as the corresponding $\underline{\Pi}_{\dot{X}}^{\mathrm{tp}} / \underline{\Pi}_{\ddot{Y}}^{\mathrm{tp}} \cong \underline{\mathbb{Z}}$-orbit $\ddot{\eta}^{\Theta}, \underline{\mathbb{Z}}$ - may be thought of as objects associated to the " $\underline{\Pi}^{\text {tp" }}$ of $\underline{\dot{X}}^{\log }, \underline{\dot{C}}^{\log }, \underline{X}^{\log }, \underline{C}^{\log }$. On the other hand, the composites of the coverings $\ddot{Y}^{\log } \rightarrow C^{\log }, Y^{\log } \rightarrow C^{\log }$ with $\underline{\underline{C}}^{\log } \rightarrow$ $C^{\log }$ determine new coverings

$$
\underline{\underline{Y}}^{\log } \rightarrow \ddot{Y}^{\log } ; \quad \underline{\underline{Y}}^{\log } \rightarrow Y^{\log }
$$

of degree $l$. Moreover, the choice of a splitting of $\bar{D}_{x} \rightarrow G_{K}$ [cf. Proposition 2.2 , (ii)] that determined the covering $\underline{\underline{X}}^{\log } \rightarrow \underline{X}^{\log }$ determines [by considering the natural map $D_{x} \rightarrow \underline{\Pi}_{\ddot{Y}}^{\mathrm{tp}} \rightarrow\left(\underline{\Pi}_{\dot{Y}}^{\mathrm{tp}}\right)^{\Theta}-$ cf. Proposition 1.5, (ii)] a specific class $\in H^{1}\left(\underline{\Pi}_{\ddot{Y}}^{\mathrm{tp}}, \underline{\Delta}_{\Theta} \otimes \mathbb{Z} / l \mathbb{Z}\right)$, which may be thought of as a choice of $\ddot{\eta}^{\Theta}$ up to an $\left(\mathcal{O}_{K}^{\times}\right)^{l}$-multiple [i.e., as opposed to only up to a $\mathcal{O}_{K}^{\times}$-multiple]. Now it is a tautology that, upon restriction to the covering $\ddot{\underline{Y}}^{\log } \rightarrow \ddot{Y}^{\log }$ [which was determined, in effect, by the choice of a splitting of $\left.\overline{\bar{D}}_{x} \rightarrow G_{K}\right]$, the class $\ddot{\eta}^{\Theta}$ determines a class

$$
\stackrel{\ddot{\ddot{\theta}}}{\Theta}^{\Theta} \in H^{1}\left(\underline{\underline{\Pi}}_{\underline{\underline{\underline{Y}}}}^{\mathrm{tp}}, l \cdot \underline{\Delta}_{\Theta}\right)
$$

— as well as a corresponding $\underline{\underline{\Pi}}_{\underline{\underline{\dot{X}}}}^{\mathrm{tp}} / \underline{\underline{\Pi}}_{\underline{\underline{\dot{Y}}}}^{\mathrm{tp}} \cong \underline{\Pi}_{\underline{\underline{X}}}^{\mathrm{tp}} / \underline{\Pi}_{\dot{Y}}^{\mathrm{tp}} \cong l \cdot \underline{\mathbb{Z}}^{\text {-orbit }} \underline{\underline{\underline{\varphi}}}^{\Theta, l \cdot \mathbb{Z}}$ - which may be thought of as objects associated to the " $\underline{\Pi}$ " of $\underline{\underline{\dot{X}}}^{\log }, \underline{\underline{\dot{C}}}^{\log }, \underline{\underline{X}}^{\log }, \underline{\underline{C}}^{\log }$, and which satisfy the following property:

$$
\left.H^{1}\left(\underline{\underline{\Pi}}_{\underline{\underline{\underline{P}}}}^{\mathrm{tp}}, l \cdot \underline{\Delta}_{\Theta}\right) \ni \underline{\underline{\underline{\eta}}}^{\Theta} \mapsto \ddot{\eta}^{\Theta}\right|_{\underline{\underline{\underline{Y}}}} \in H^{1}\left(\underline{\underline{\Pi}}_{\underline{\underline{\underline{Y}}}}^{\mathrm{tp}}, \underline{\Delta}_{\Theta}\right)
$$

[relative to the natural inclusion $l \cdot \underline{\Delta}_{\Theta} \hookrightarrow \underline{\Delta}_{\Theta}$ ]. That is to say, at a more intuitive level, $\ddot{\underline{\varphi}}^{\Theta}$ may be thought of as an "l-th root of the étale theta function". In the following, we shall also consider the $l \cdot \underline{\mathbb{Z}}$-orbit $\ddot{\eta}^{\Theta, l \cdot \underline{\mathbb{Z}}}$ of $\ddot{\eta}^{\Theta}$, as well as the $\underline{\underline{\Pi}} \underline{\underline{X}}_{\underline{\underline{X}}}^{\mathrm{tp}} / \underline{\Pi}_{\underline{\underline{Y}}}^{\mathrm{tp}} \cong \underline{\Pi_{\underline{X}}^{\mathrm{p}}} / \underline{\Pi}_{\tilde{Y}}^{\mathrm{tp}} \cong\left\{(l \cdot \underline{\mathbb{Z}}) \times \boldsymbol{\mu}_{2}\right\}$-orbits

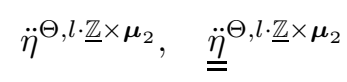

of $\ddot{\eta}^{\Theta}, \underline{\underline{\eta}}^{\Theta}$, and the $\underline{\Pi}_{X}^{\mathrm{tp}} / \underline{\Pi}_{\ddot{Y}}^{\mathrm{tp}} \cong\left(\underline{\mathbb{Z}} \times \boldsymbol{\mu}_{2}\right)$-orbit $\ddot{\eta}^{\Theta}, \underline{\mathbb{Z}} \times \boldsymbol{\mu}_{2}$ of $\ddot{\eta}^{\Theta}$.

Definition 2.7. If $\ddot{\eta}^{\Theta,} \underline{\mathbb{Z}}$ is of standard type, then we shall also refer to

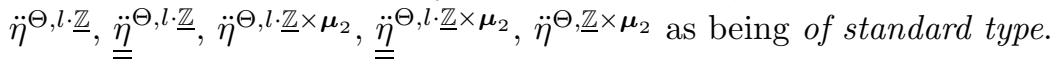

Corollary 2.8 (Constant Multiple Rigidity of Roots of the Étale Theta Function). For $\square=\alpha, \beta$, let us assume that we have smooth log orbicurves as in the above discussion, over a finite extension $K_{\square}$ of $\mathbb{Q}_{p}$. Let

$$
\begin{aligned}
& \gamma: \underline{\Pi}_{\underline{\underline{X}}_{\alpha}}^{\mathrm{tp}} \stackrel{\sim}{\rightarrow} \underline{\Pi}_{\underline{\underline{X}}_{\beta}}^{\mathrm{tp}} \text { (respectively, } \gamma: \underline{\Pi}_{\underline{X}_{\alpha}}^{\mathrm{tp}} \stackrel{\sim}{\rightarrow} \underline{\Pi}_{\underline{X}_{\beta}}^{\mathrm{tp}} ; \\
& \left.\gamma: \underline{\Pi}_{\underline{\underline{C}}}^{\mathrm{tp}} \stackrel{\sim}{\rightarrow} \underline{\Pi}_{\underline{\underline{C}}_{\beta}}^{\mathrm{tp}} ; \gamma: \underline{\Pi}_{\underline{C}_{\alpha}}^{\mathrm{tp}} \stackrel{\sim}{\rightarrow} \underline{\Pi}_{\underline{\Pi}_{\beta}}^{\mathrm{tp}}\right)
\end{aligned}
$$


be an isomorphism of topological groups. Then:

(i) The isomorphism $\gamma$ preserves the property $[$ cf. Theorem 1.6, (iii)] that $\ddot{\eta}_{\square}^{\Theta}, l \cdot \underline{\mathbb{Z}} \times \boldsymbol{\mu}_{2}$ (respectively, $\ddot{\eta}_{\square}^{\Theta, \underline{Z} \times \boldsymbol{\mu}_{2}} ; \ddot{\eta}_{\square}^{\Theta, l \cdot \underline{\mathbb{Z}} \times \boldsymbol{\mu}_{2}} ; \ddot{\eta}_{\square}^{\Theta, l \cdot \underline{\mathbb{Z}} \times \boldsymbol{\mu}_{2}}$ ) be of standard type $\bar{a}$ property that determines this collection of classes up to multiplication by a root of unity of order $l$ (respectively, $1 ; l ; 1)$.

(ii) Suppose further that the cusps of $\underline{\underline{X}}_{\square}$ are rational over $K_{\square}$, that the residue characteristic of $K_{\square}$ is prime to $l$, and that $K_{\square}$ contains a primitive $l$-th root of unity. Then the $\{ \pm 1\}$-[i.e., $\left.\boldsymbol{\mu}_{2}\right]$ structure of Theorem 1.10, (iii), determines a $\boldsymbol{\mu}_{2 l}$ (respectively, $\boldsymbol{\mu}_{2} ; \boldsymbol{\mu}_{2 l} ; \boldsymbol{\mu}_{2}$ )-structure [cf. [Mzk13], Corollary 4.12] on the $\left(K_{\square}^{\times}\right)^{\wedge}$-torsor at the cusps of $\underline{\underline{X}}_{\square}^{\log }$ (respectively, $\underline{X}_{\square}^{\log } ; \underline{\underline{X}}_{\square}^{\log } ; \underline{X}_{\square}^{\log }$ ). Moreover, this $\boldsymbol{\mu}_{2 l}$ (respectively, $\boldsymbol{\mu}_{2} ; \boldsymbol{\mu}_{2 l} ; \boldsymbol{\mu}_{2}$ )-structure is compatible with the canonical integral structure [cf. [Mzk13], Definition 4.1, (iii)] determined by the stable model of $X_{\square}^{\log }$ and preserved by $\gamma$.

(iii) If the data for $\square=\alpha, \beta$ are equal, and $\gamma$ arises [cf. Proposition 2.6] from an inner automorphism of $\underline{\Pi}_{\underline{\underline{\dot{X}}}}^{\mathrm{tp}}$ (respectively, $\underline{\underline{\Pi}}_{\underline{\underline{X}}_{\square}^{\mathrm{tp}}} ; \underline{\Pi}_{\underline{\underline{\dot{C}}}}^{\mathrm{tp}} ; \underline{\Pi}_{\underline{\underline{C}}_{\square}}^{\mathrm{tp}}$ ),

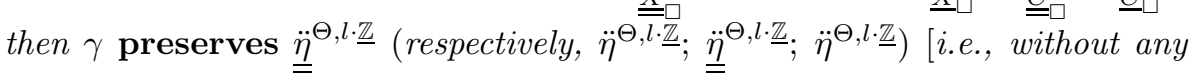
constant multiple indeterminacy].

Proof. First, let us recall the characteristic nature of the various coverings involved [cf. Propositions 2.4, 2.6]. Now assertion (i) follows immediately from Theorem 1.10, (i), and the definitions; assertion (ii) follows immediately from Theorem 1.10, (iii), and the definitions; assertion (iii) follows immediately from Remark 1.9.1.

Before proceeding, we pause to take a closer look at the cusps of the various smooth log orbicurves under consideration. First, we recall from the discussion preceding Lemma 1.2 that the irreducible components of the special fiber of $\mathfrak{Y}^{\log }$ may be assigned labels $\in \mathbb{Z}$, in a natural fashion. These labels thus determine labels $\in \mathbb{Z}$ for the cusps of $Y^{\log }$ [i.e., by considering the irreducible component of the special fiber of $\mathfrak{Y}^{\log }$ that contains the closure in $\mathfrak{Y}$ of the cusp in question]. Moreover, by considering the covering $\underline{\underline{\underline{Y}}}^{\log } \rightarrow Y^{\log }$, we thus obtain labels $\in \mathbb{Z}$ for the cusps of $\underline{\underline{\underline{Y}}}^{\log }$. Since the various smooth log orbicurves

$$
\underline{\underline{X}}^{\log } ; \quad \underline{\dot{X}}^{\log } ; \quad \underline{\underline{\dot{C}}}^{\log } ; \quad \underline{\dot{C}}^{\log } ; \quad \underline{\underline{X}}^{\log ;} \quad \underline{X}^{\log } ; \quad \underline{\underline{C}}^{\log } ; \quad \underline{C}^{\log }
$$

all appear as subcoverings of the covering $\underline{\underline{Y}}^{\log } \rightarrow X^{\log }$, we thus obtain labels $\in \mathbb{Z}$ for the cusps of these smooth log orbicurves, which are well-defined up to a certain indeterminacy. If we write

$$
(\mathbb{Z} / l \mathbb{Z})_{ \pm}
$$


for the quotient of the set $\mathbb{Z} / l \mathbb{Z}$ by the natural multiplicative action of \pm 1 , then it follows immediately from the construction of these smooth log orbicurves that this indeterminacy is such that the labels for the cusps of these smooth $\log$ orbicurves may be thought of as well-defined elements of $(\mathbb{Z} / l \mathbb{Z})_{ \pm}$.

Corollary 2.9 (Labels of Cusps). Suppose that $K$ contains a primitive $l$-th root of unity. Then for each of the smooth log orbicurves

$$
\underline{\underline{\dot{X}}}^{\log } ; \quad \underline{\underline{\dot{C}}}^{\log } ; \quad \underline{\dot{C}}^{\log } ; \quad \underline{\underline{X}}^{\log } ; \quad \underline{\underline{C}}^{\log } ; \quad \underline{C}^{\log }
$$

[as defined in the above discussion], the labels of the above discussion determine $a$ bijection of the set

$$
(\mathbb{Z} / l \mathbb{Z})_{ \pm}
$$

with the set of "Aut ${ }_{K}(-)$ "-orbits [cf. Remark 2.6.1] of the cusps of the smooth log orbicurve. Moreover, in the case of $\underline{\underline{X}}^{\log }, \underline{\underline{C}}^{\log }$, and $\underline{C}^{\log }$, these bijections are preserved by arbitrary isomorphisms of topological groups " $\gamma$ " as in Corollary 2.8 .

Proof. The asserted bijections follow immediately by tracing through the definitions of the various smooth log orbicurves [cf. Remark 2.6.1]. With regard to showing that these bijections are preserved by " $\gamma$ " as in Corollary 2.8, we reduce immediately by Proposition 2.4 to the case of $\underline{C}^{\log }$; in this case, the desired preservation follows immediately from the definition of the labels in question in the discussion above, together with the fact that such $\gamma$ always preserve the dual graphs of the special fibers of the orbicurves in question [cf. [Mzk2], Lemma 2.3].

Remark 2.9.1. We observe in passing that a bijection as in Corollary 2.9 fails to hold for $\underline{\dot{X}}^{\log }, \underline{X}^{\log }$ - cf. Remark 2.6.1.

Remark 2.9.2. In the situation of Corollary 2.8, (ii), we make the following observation, relative to the labels of Corollary 2.9: The $2 l$ (respectively, 2) trivializations of the $\left(K_{\square}^{\times}\right)^{\wedge}$-torsor at a cusp labeled 0 (respectively, an arbitrary cusp) of $\underline{\underline{X}}_{\square}^{\log }$ (respectively, $\underline{X}_{\square}^{\log }$ ) determined by the $\boldsymbol{\mu}_{2 l}$ (respectively, $\boldsymbol{\mu}_{2}$ )-structure under discussion are permuted transitively by the subgroup of $\operatorname{Aut}_{K_{\square}}\left(\underline{\underline{X}}_{\square}^{\text {log }}\right)$ (respectively, $\operatorname{Aut}_{K_{\square}}\left(\underline{X}_{\square}^{\text {log }}\right)$ ) [cf. Remarks 2.1.1, 2.6.1, 2.9.1] that stabilizes the cusp. In the case of $\underline{\underline{X}}_{\square}^{\log }$, at cusps with nonzero labels, the subgroup of the corresponding "Aut $K_{\square}(-)$ " that stabilizes the cusp permutes the $2 l$ trivializations under consideration via the action of $\boldsymbol{\mu}_{l}$ [hence has precisely two orbits]. 
Next, let $N \geq 1$ be an integer; set

$$
\Delta_{\boldsymbol{\mu}_{N}} \stackrel{\text { def }}{=}(\mathbb{Z} / N \mathbb{Z}(1)) ; \quad \Pi_{\boldsymbol{\mu}_{N}, K} \stackrel{\text { def }}{=} \Delta_{\boldsymbol{\mu}_{N}} \rtimes G_{K}
$$

- so we have a natural exact sequence $1 \rightarrow \Delta_{\boldsymbol{\mu}_{N}} \rightarrow \Pi_{\boldsymbol{\mu}_{N}, K} \rightarrow G_{K} \rightarrow 1$.

Definition 2.10. If $\Pi \rightarrow G_{K}$ is a topological group equipped with an augmentation [i.e., a surjection] to $G_{K}$, then we shall write

$$
\Pi\left[\boldsymbol{\mu}_{N}\right] \stackrel{\text { def }}{=} \Pi \times_{G_{K}} \Pi_{\boldsymbol{\mu}_{N}, K}
$$

and refer to $\Pi\left[\boldsymbol{\mu}_{N}\right]$ as the cyclotomic envelope of $\Pi \rightarrow G_{K}$ [or $\Pi$, for short]. Also, if $\Delta \stackrel{\text { def }}{=} \operatorname{Ker}\left(\Pi \rightarrow G_{K}\right)$, then we shall write

$$
\Delta\left[\boldsymbol{\mu}_{N}\right] \stackrel{\text { def }}{=} \operatorname{Ker}\left(\Pi\left[\boldsymbol{\mu}_{N}\right] \rightarrow G_{K}\right)
$$

- so $\Delta\left[\boldsymbol{\mu}_{N}\right]=\Delta \times \Delta_{\boldsymbol{\mu}_{N}} ;$ we have a natural exact sequence $1 \rightarrow \Delta\left[\boldsymbol{\mu}_{N}\right] \rightarrow$ $\Pi\left[\boldsymbol{\mu}_{N}\right] \rightarrow G_{K} \rightarrow 1$. Note that, by construction, we have a tautological section $G_{K} \rightarrow \Pi_{\boldsymbol{\mu}_{N}, K}$ of $\Pi_{\boldsymbol{\mu}_{N}, K} \rightarrow G_{K}$, which determines a section

$$
s_{\Pi}^{\mathrm{alg}}: \Pi \rightarrow \Pi\left[\boldsymbol{\mu}_{N}\right]
$$

of $\Pi\left[\boldsymbol{\mu}_{N}\right] \rightarrow \Pi$, which we shall also call tautological. We shall refer to a $\boldsymbol{\mu}_{N^{-}}$ orbit, relative to the action of $\boldsymbol{\mu}_{N}$ by conjugation, of objects associated to $\Pi\left[\boldsymbol{\mu}_{N}\right]$ [e.g., subgroups of $\Pi\left[\boldsymbol{\mu}_{N}\right]$, homomorphisms from $\Pi\left[\boldsymbol{\mu}_{N}\right]$ to another topological group, etc.] as a $\boldsymbol{\mu}_{N}$-conjugacy class.

Proposition 2.11 (General Properties of the Cyclotomic Envelope). For $\square=\alpha, \beta$, let $\Pi_{\square} \rightarrow G_{K_{\square}}$ be an open subgroup of either the tempered or the profinite fundamental group of a hyperbolic orbicurve over a finite extension $K_{\square}$ of $\mathbb{Q}_{p}$; write $\Delta_{\square}$ for the kernel of the natural morphism $\Pi_{\square} \rightarrow G_{K_{\square}}$. Then:

(i) The kernel of the natural surjection $\Delta_{\square}\left[\boldsymbol{\mu}_{N}\right] \rightarrow \Delta_{\square}$ is equal to the center of $\Delta_{\square}\left[\boldsymbol{\mu}_{N}\right]$. In particular, any isomorphism of topological groups $\Delta_{\alpha}\left[\boldsymbol{\mu}_{N}\right] \stackrel{\sim}{\rightarrow}$ $\Delta_{\beta}\left[\boldsymbol{\mu}_{N}\right]$ is compatible with the natural surjections $\Delta_{\square}\left[\boldsymbol{\mu}_{N}\right] \rightarrow \Delta_{\square}$.

(ii) The kernel of the natural surjection $\Pi_{\square}\left[\boldsymbol{\mu}_{N}\right] \rightarrow \Pi_{\square}$ is equal to the union of the centralizers of the open subgroups of $\Pi_{\square}\left[\boldsymbol{\mu}_{N}\right]$. In particular, any isomorphism of topological groups $\Pi_{\alpha}\left[\boldsymbol{\mu}_{N}\right] \stackrel{\sim}{\rightarrow} \Pi_{\beta}\left[\boldsymbol{\mu}_{N}\right]$ is compatible with the natural surjections $\Pi_{\square}\left[\boldsymbol{\mu}_{N}\right] \rightarrow \Pi_{\square}$.

Proof. Assertions (i), (ii) follow immediately from the "temp-slimness" [i.e., the triviality of the centralizers of all open subgroups of $] \Delta_{\square}, \Pi_{\square}[\mathrm{cf}$. [Mzk14], Example 3.10]. 
Next, let us write

$$
\underline{\Pi}_{C}^{\Theta} \stackrel{\text { def }}{=} \underline{\Pi}_{C} / \operatorname{Ker}\left(\underline{\Delta}_{X} \rightarrow \underline{\Delta}_{X}^{\Theta}\right) ; \quad \underline{\Delta}_{C}^{\Theta} \stackrel{\text { def }}{=} \underline{\Delta}_{C} / \operatorname{Ker}\left(\underline{\Delta}_{X} \rightarrow \underline{\Delta}_{X}^{\Theta}\right)
$$

and, in a similar vein, denote by means of a superscript " $\Theta$ " the quotients of the tempered and profinite fundamental groups of $\underline{\underline{X}}^{\log }, \underline{\dot{X}}^{\log }, \dot{X} \log , \underline{\underline{X}}^{\log }, \underline{X}^{\log }$, $X^{\log }, \underline{\dot{C}}^{\log }, \underline{\dot{C}}^{\log }, \dot{C}^{\log }, \underline{\underline{C}}^{\log }, \underline{C}^{\log }, C^{\log }$, determined by these quotients. Also, let us write

$$
\underline{\Pi}_{C}^{\text {ell def }} \stackrel{\Pi_{C}}{=} \operatorname{Ker}\left(\underline{\Delta}_{X} \rightarrow \underline{\Delta}_{X}^{\text {ell }}\right) ; \quad \underline{\Delta}_{C}^{\text {ell }} \stackrel{\text { def }}{=} \underline{\Delta}_{C} / \operatorname{Ker}\left(\underline{\Delta}_{X} \rightarrow \underline{\Delta}_{X}^{\text {ell }}\right)
$$

and denote by means of a superscript "ell" the various induced quotients.

Proposition 2.12 (The Cyclotomic Envelope of the Theta Quotient). Let $\Delta_{*}$ be one of the following topological groups:

$$
\underline{\underline{\Delta}}_{\underline{\underline{X}}}^{\mathrm{tp}} ; \quad \underline{\underline{\Delta}}_{\underline{\underline{\underline{C}}}}^{\mathrm{tp}} ; \quad \underline{\underline{\Delta}}_{\underline{\underline{C}}}^{\mathrm{tp}} ; \quad \underline{\underline{\Delta}}_{\underline{\underline{X}}} ; \quad \underline{\underline{\Delta}}_{\underline{\underline{\underline{C}}}} ; \quad \underline{\underline{\Delta}}_{\underline{\underline{C}}}
$$

Then:

(i) We have an inclusion

$$
\operatorname{Ker}\left(\Delta_{*}^{\Theta} \rightarrow \Delta_{*}^{\mathrm{ell}}\right)=l \cdot \underline{\Delta}_{\Theta} \subseteq\left[\Delta_{*}^{\Theta}, \Delta_{*}^{\Theta}\right]
$$

of subgroups of $\Delta_{*}^{\Theta}$.

(ii) The intersection

$$
\left[\Delta_{*}^{\Theta}\left[\boldsymbol{\mu}_{N}\right], \Delta_{*}^{\Theta}\left[\boldsymbol{\mu}_{N}\right]\right] \bigcap\left(l \cdot \underline{\Delta}_{\Theta}\right)\left[\boldsymbol{\mu}_{N}\right] \subseteq\left(l \cdot \underline{\Delta}_{\Theta}\right)\left[\boldsymbol{\mu}_{N}\right] \subseteq \Delta_{*}^{\Theta}\left[\boldsymbol{\mu}_{N}\right]
$$

coincides with the image of the restriction of the tautological section of $\Delta_{*}^{\Theta}\left[\boldsymbol{\mu}_{N}\right] \rightarrow \Delta_{*}^{\Theta}$ to $l \cdot \underline{\Delta}_{\Theta}$.

Proof. First, we consider the inclusion of assertion (i). Now since $l$ is odd, the prime-to-2 portion of this inclusion then follows immediately from the well-known structure of the "theta-group" $\left(\underline{\Delta}_{X}^{\mathrm{tp}}\right)^{\Theta}$ [cf. also the definition of the covering $\left.\underline{\underline{C}}^{\log } \rightarrow C^{\log }\right]$; in the case of $\underline{\underline{X}}^{\log }, \underline{\underline{C}}^{\log }$, the pro-2 portion of this inclusion follows similarly. On the other hand, in the case of $\underline{\dot{C}}^{\log }$, the pro-2 portion of this inclusion follows from the fact that, in the notation of Remark 2.2.1 [i.e., more precisely, when " $l=2$ "], if we denote by $\epsilon_{\mathbb{Z}}^{\Delta}, \epsilon_{\mu}^{\Delta} \in$ $\bar{\Delta}_{X}\left(\subseteq \bar{\Delta}_{C}\right), \epsilon_{ \pm}^{\Delta} \in \bar{\Delta}_{C}$ liftings to $\bar{\Delta}_{C}$ of the elements of $\bar{\Delta}_{C}^{\text {ell }}$ determined by the automorphisms " $\epsilon_{\mathbb{Z}}$ ", " $\epsilon_{\mu}$ ", " $\epsilon_{ \pm}$" of the discussion preceding Definition 1.7, 
then $\epsilon_{ \pm}^{\Delta}$ commutes with $\epsilon_{\mathbb{Z}}^{\Delta}, \epsilon_{\mu}^{\Delta}$ [cf. the observation of Remark 2.2.1], so the commutator

$$
\left[\epsilon_{\mathbb{Z}}^{\Delta}, \epsilon_{ \pm}^{\Delta} \cdot \epsilon_{\mu}^{\Delta}\right]=\left[\epsilon_{\mathbb{Z}}^{\Delta}, \epsilon_{\mu}^{\Delta}\right]
$$

is a nonzero element of $\bar{\Delta}_{\Theta}$. Assertion (ii) follows formally from assertion (i).

Remark 2.12.1. Note that the inclusion of Proposition 2.12, (i) — which will be crucial in the theory to follow - fails to hold if one replaces $\underline{\underline{X}}^{\log }, \underline{\underline{C}}^{\log }$, $\underline{\underline{C}}^{\log }$ by $\underline{\underline{X}}^{\log }, \dot{X}^{\log }$ [one has problems at the prime 2$] ; \underline{\dot{X}}^{\log }$ [one has problems at the prime 2 and the primes dividing $l] ; \underline{X}^{\log }, \underline{\dot{C}}^{\log }, \underline{C}^{\log }$ [one has problems at the primes dividing $l]$. (There is no problem, however, if one replaces $\underline{\underline{X}}^{\log }$, $\underline{\underline{C}}^{\log }, \underline{\underline{C}}^{\log }$ by $X^{\log }, \dot{C}^{\log }, C^{\log }$ since this just corresponds to the case $l=1$.) Indeed, the original motivation for the introduction of the slightly complicated coverings $\underline{\underline{X}}^{\log }, \underline{\underline{C}}^{\log }, \underline{\underline{C}}^{\log }$ was precisely to avoid these problems.

Next, let us observe that, by subtracting [i.e., if we treat cohomology classes additively] the reduction modulo $N$ of any member of the collection of [cocycles

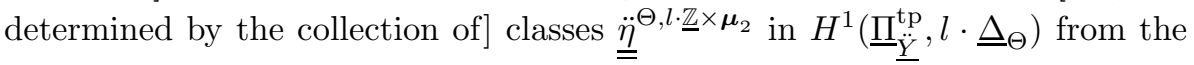
[composite with the inclusion into $\underline{\underline{\Pi}}_{\underline{\underline{Y}}}^{\mathrm{tp}}\left[\boldsymbol{\mu}_{N}\right]$ of the] tautological section

$$
s_{\underline{\underline{\underline{Y}}}}^{\text {alg }} \stackrel{\text { def }}{=} s_{\underline{\underline{\underline{\underline{M}}}}}^{\mathrm{alg}}: \underline{\Pi}_{\underline{\underline{\underline{Y}}}}^{\mathrm{tp}} \rightarrow \underline{\Pi}_{\underline{\underline{\underline{Y}}}}^{\mathrm{tp}}\left[\boldsymbol{\mu}_{N}\right] \hookrightarrow \underline{\Pi}_{\underline{\underline{Y}}}^{\mathrm{tp}}\left[\boldsymbol{\mu}_{N}\right]
$$

— where we apply the natural isomorphism $\boldsymbol{\mu}_{N} \cong\left(l \cdot \underline{\Delta}_{\Theta}\right) \otimes(\mathbb{Z} / N \mathbb{Z})$ - yields a new homomorphism: $s_{\underline{\underline{\underline{Y}}}}^{\Theta}: \underline{\underline{\Pi}}_{\underline{\underline{\underline{Y}}}}^{\mathrm{tp}} \rightarrow \underline{\Pi}_{\underline{\underline{Y}}}^{\mathrm{tp}}\left[\boldsymbol{\mu}_{N}\right]$.

Now since the tautological section $s_{\ddot{Y}}^{\text {alg }}$ extends to a tautological section $s_{\underline{\Pi}_{C}^{\mathrm{tp}}}^{\mathrm{alg}}: \underline{\Pi}_{\underline{\underline{C}}}^{\mathrm{tp}} \rightarrow \underline{\Pi}_{\underline{\underline{C}}}^{\mathrm{tp}}\left[\boldsymbol{\mu}_{N}\right]\left[\right.$ where we regard $\underline{\Pi}_{\underline{\underline{Y}}}^{\underline{\mathrm{tp}}}\left[\boldsymbol{\mu}_{N}\right]$ as a subgroup of $\left.\underline{\Pi}_{\underline{\underline{C}}}^{\mathrm{tp}}\left[\boldsymbol{\mu}_{N}\right]\right]$, it follows that the natural outer action

$$
\operatorname{Gal}(\underline{\underline{Y}} / \underline{\underline{C}}) \cong \underline{\underline{\Pi}} \underline{\underline{C}}_{\underline{\mathrm{tp}}} / \underline{\underline{Y}}_{\underline{\underline{Y}}}^{\mathrm{tp}} \cong \underline{\underline{\Pi}}_{\underline{\underline{\mathrm{C}}}}^{\mathrm{tp}}\left[\boldsymbol{\mu}_{N}\right] / \underline{\underline{\Pi}}_{\underline{\underline{Y}}}^{\mathrm{tp}}\left[\boldsymbol{\mu}_{N}\right] \hookrightarrow \operatorname{Out}\left(\underline{\Pi}_{\underline{\underline{Y}}}^{\mathrm{tp}}\left[\boldsymbol{\mu}_{N}\right]\right)
$$

of $\operatorname{Gal}(\underline{\underline{Y}} / \underline{\underline{C}})$ on $\underline{\Pi}_{\underline{\underline{Y}}}^{\mathrm{tp}}\left[\boldsymbol{\mu}_{N}\right]$ fixes the image of $s_{\underline{\underline{Y}}}^{\text {alg }}$, up to conjugation by an element of $\boldsymbol{\mu}_{N}$. In particular, it follows immediately $\overline{\overline{\text { from }}}$ the definitions that the various $s_{\underline{\underline{\underline{Y}}}}^{\Theta}$ that arise from different choices of [a cocycle contained in] a class $\in \underline{\underline{\ddot{q}}}^{\Theta, l \cdot \underline{\mathbb{Z}} \times \boldsymbol{\mu}_{2}}$ are obtained as $\underline{\Pi}_{\underline{X}}^{\mathrm{tp}}\left[\boldsymbol{\mu}_{N}\right]$-conjugates [where we recall that we have a natural isomorphism $\left.(\underline{\underline{\underline{\Pi}}} \underset{\underline{\underline{X}}}{\mathrm{tp}} \rightarrow) \operatorname{Gal}(\underline{\underline{\underline{Y}}} / \underline{\underline{X}}) \stackrel{\sim}{\rightarrow}(l \cdot \underline{\mathbb{Z}}) \times \boldsymbol{\mu}_{2}\right]$ of any given $s_{\underline{\underline{\underline{Y}}}}^{\Theta}$. [Here, we 
note that "conjugation by an element of $\boldsymbol{\mu}_{N}$ " corresponds precisely to modifying a cocycle by a coboundary.]

Note, moreover, that we have a natural outer action

$$
K^{\times} \rightarrow\left(K^{\times}\right) /\left(K^{\times}\right)^{N} \stackrel{\sim}{\rightarrow} H^{1}\left(G_{K}, \boldsymbol{\mu}_{N}\right) \hookrightarrow H^{1}\left(\underline{\Pi}_{\underline{\underline{Y}}}^{\mathrm{tp}}, \boldsymbol{\mu}_{N}\right) \rightarrow \operatorname{Out}\left(\underline{\Pi}_{\underline{\underline{Y}}}^{\mathrm{tp}}\left[\boldsymbol{\mu}_{N}\right]\right)
$$

- where the " $\stackrel{\sim}{\rightarrow}$ " is the Kummer map - of $K^{\times}$on $\underline{\Pi}_{Y}^{\mathrm{tp}}\left[\boldsymbol{\mu}_{N}\right]$, which induces the trivial outer action on both the quotient $\underline{\underline{\Pi}}_{\underline{\underline{Y}}}^{\mathrm{tp}}\left[\boldsymbol{\mu}_{N}\right] \rightarrow \underline{\underline{\Pi}}_{\underline{\underline{Y}}}^{\mathrm{tp}}$ and the kernel of this quotient. Relative to this natural outer action, replacing $\underline{\ddot{\eta}}^{\Theta, l \cdot \underline{\mathbb{Z}} \times \boldsymbol{\mu}_{2}}$ by an $\mathcal{O}_{K}^{\times}$-multiple of $\underline{\underline{\underline{\eta}}}^{\Theta, l \cdot \underline{\mathbb{Z}} \times \boldsymbol{\mu}_{2}}$ [cf. Proposition 1.3] corresponds to replacing $s_{\underline{\underline{\underline{Y}}}}^{\Theta}$ by an $\mathcal{O}_{K}^{\times}$-conjugate of $s_{\ddot{\underline{Y}}}^{\Theta}$.

Definition 2.13. In the notation of the above discussion:

(i) Write

$$
\mathcal{D}_{\underline{\underline{Y}}} \subseteq \operatorname{Out}\left(\underline{\underline{\Pi}}_{\underline{\underline{Y}}}^{\mathrm{tp}}\left[\boldsymbol{\mu}_{N}\right]\right)
$$

for the subgroup of $\operatorname{Out}\left(\underline{\Pi}_{\underline{Y}}^{\mathrm{tp}}\left[\boldsymbol{\mu}_{N}\right]\right)$ generated by the image of $K^{\times}, \operatorname{Gal}(\underline{\underline{Y}} / \underline{\underline{X}})(\cong$ $l \cdot \underline{\mathbb{Z}})$. We shall refer to $s_{\underline{\underline{Y}}}^{\stackrel{\Theta}{\Theta}}: \underline{\Pi}_{\underline{\underline{Y}}}^{\mathrm{tp}} \rightarrow \underline{\Pi}_{\underline{\underline{Y}}}^{\mathrm{tp}}\left[\boldsymbol{\mu}_{N}\right]$ as the $[\bmod N]$ theta section. We shall refer to $s_{\underline{\underline{\underline{Y}}}}^{\mathrm{alg}}: \underline{\Pi}_{\underline{\underline{\underline{Y}}}}^{\mathrm{tp}} \rightarrow \underline{\underline{\Pi}}_{\underline{\underline{Y}}}^{\mathrm{tp}}\left[\boldsymbol{\mu}_{N}\right]$ as the $[\bmod N]$ algebraic section.

(ii) We shall refer to as a $[\bmod N]$ model mono-theta environment any [ordered] collection of data as folows:

(a) the topological group $\underline{\underline{\Pi}}_{\underline{\underline{Y}}}^{\mathrm{tp}}\left[\boldsymbol{\mu}_{N}\right]$;

(b) the subgroup $\mathcal{D}_{\underline{\underline{Y}}} \subseteq \operatorname{Out}\left(\underline{\Pi}_{\underline{\underline{Y}}}^{\mathrm{tp}}\left[\boldsymbol{\mu}_{N}\right]\right)$;

(c) the $\boldsymbol{\mu}_{N}$-conjugacy class of subgroups $\subseteq \underline{\Pi}_{\underline{\underline{Y}}}^{\mathrm{tp}}\left[\boldsymbol{\mu}_{N}\right]$ determined by the image of the theta section $s_{\underline{\underline{Y}}}^{\Theta}$.

We shall refer to as a $[\bmod N]$ mono-theta environment any [ordered] collection of data consisting of a topological group $\Pi$, a subgroup $\mathcal{D}_{\Pi} \subseteq \operatorname{Out}(\Pi)$, and a collection of subgroups $s_{\Pi}^{\Theta}$ of $\Pi$ such that there exists an isomorphism of topological groups $\Pi \stackrel{\sim}{\rightarrow} \underline{\Pi}_{\underline{\underline{Y}}}^{\mathrm{tp}}\left[\boldsymbol{\mu}_{N}\right]\left[\mathrm{cf}\right.$. (a)] mapping $\mathcal{D}_{\Pi} \subseteq \operatorname{Out}(\Pi)$ to $\mathcal{D}_{\underline{\underline{Y}}}[\mathrm{cf}$. (b)] and $s_{\Pi}^{\Theta}$ to the $\boldsymbol{\mu}_{N^{-}}$-conjugacy class of (c). [In particular, every model monotheta environment determines a mono-theta environment.] We shall refer to as an isomorphism of $[\bmod N]$ mono-theta environments $\mathbb{M} \stackrel{\sim}{\rightarrow} \mathbb{M}^{\prime}$ between two $[\bmod N]$ mono-theta environments

$$
\mathbb{M} \stackrel{\text { def }}{=}\left(\Pi, \mathcal{D}_{\Pi}, s_{\Pi}^{\Theta}\right) ; \quad \mathbb{M}^{\prime} \stackrel{\text { def }}{=}\left(\Pi^{\prime}, \mathcal{D}_{\Pi^{\prime}}, s_{\Pi^{\prime}}^{\Theta}\right)
$$


any isomorphism of topological groups $\Pi \stackrel{\sim}{\rightarrow} \Pi^{\prime}$ that maps $\mathcal{D}_{\Pi} \mapsto \mathcal{D}_{\Pi^{\prime}}, s_{\Pi}^{\Theta} \mapsto$ $s_{\Pi^{\prime}}^{\ominus}$. If $N^{\prime} \mid N, \mathbb{M}$ is a $\bmod N$ mono-theta environment, and $\mathbb{M}^{\prime}$ is a $\bmod N^{\prime}$ mono-theta environment, then we shall refer to as a morphism of mono-theta environments $\mathbb{M} \rightarrow \mathbb{M}^{\prime}$ any isomorphism $\mathbb{M}_{N^{\prime}} \stackrel{\sim}{\rightarrow} \mathbb{M}^{\prime}$, where we write $\mathbb{M}_{N^{\prime}}$ for the $\bmod N^{\prime}$ mono-theta environment induced by $\mathbb{M}$.

(iii) We shall refer to as a [mod $N]$ model bi-theta environment any [ordered] collection of data as follows:

(a) the topological group $\underline{\Pi}_{\underline{\underline{Y}}}^{\mathrm{tp}}\left[\boldsymbol{\mu}_{N}\right]$;

(b) the subgroup $\mathcal{D}_{\underline{\underline{Y}}} \subseteq \operatorname{Out}\left(\underline{\Pi}_{\underline{\underline{Y}}}^{\mathrm{tp}}\left[\boldsymbol{\mu}_{N}\right]\right)$;

(c) the $\boldsymbol{\mu}_{N^{-}}$-conjugacy class of subgroups $\subseteq \underline{\Pi}_{\underline{\underline{Y}}}^{\mathrm{tp}}\left[\boldsymbol{\mu}_{N}\right]$ determined by the image of the theta section $s_{\underline{\underline{Y}}}^{\Theta}$;

(d) the $\boldsymbol{\mu}_{N^{-}}$-conjugacy class of subgroups $\subseteq \underline{\Pi}_{\underline{\underline{Y}}}^{\mathrm{tp}}\left[\boldsymbol{\mu}_{N}\right]$ determined by the image of the algebraic section $s_{\underline{\underline{\underline{Y}}}}^{\text {alg }}$.

We shall refer to as a $[\bmod N]$ bi-theta environment any collection of data consisting of a topological group $\Pi$, a subgroup $\mathcal{D}_{\Pi} \subseteq \operatorname{Out}(\Pi)$, and an ordered pair of collections of subgroups $s_{\Pi}^{\Theta}, s_{\Pi}^{\text {alg }}$ of $\Pi$ such that there exists an isomorphism of topological groups $\Pi \stackrel{\sim}{\rightarrow} \underline{\Pi}_{\underline{\underline{\underline{Y}}}}^{\mathrm{tp}}\left[\boldsymbol{\mu}_{N}\right]\left[\right.$ cf. (a)] mapping $\mathcal{D}_{\Pi} \subseteq \operatorname{Out}(\Pi)$ to $\mathcal{D}_{\underline{\underline{Y}}}$ [cf. (b)], $s_{\Pi}^{\Theta}$ to the $\boldsymbol{\mu}_{N^{-}}$-conjugacy class of (c), and $s_{\Pi}^{\text {alg }}$ to the $\boldsymbol{\mu}_{N^{-}}$-conjugacy class of (d). [In particular, every model bi-theta environment determines a bitheta environment.] We shall refer to as an isomorphism of $[\bmod N]$ bi-theta environments $\mathbb{B} \stackrel{\sim}{\rightarrow} \mathbb{B}^{\prime}$ between two $[\bmod N]$ bi-theta environments

$$
\mathbb{B} \stackrel{\text { def }}{=}\left(\Pi, \mathcal{D}_{\Pi}, s_{\Pi}^{\Theta}, s_{\Pi}^{\text {alg }}\right) ; \quad \mathbb{B}^{\prime} \stackrel{\text { def }}{=}\left(\Pi^{\prime}, \mathcal{D}_{\Pi^{\prime}}, s_{\Pi^{\prime}}^{\Theta}, s_{\Pi^{\prime}}^{\text {alg }}\right)
$$

any isomorphism of topological groups $\Pi \stackrel{\sim}{\rightarrow} \Pi^{\prime}$ that maps $\mathcal{D}_{\Pi} \mapsto \mathcal{D}_{\Pi^{\prime}}, s_{\Pi}^{\Theta} \mapsto$ $s_{\Pi^{\prime}}^{\ominus}, s_{\Pi}^{\text {alg }} \mapsto s_{\Pi^{\prime}}^{\text {alg }}$. If $N^{\prime} \mid N, \mathbb{B}$ is a $\bmod N$ bi-theta environment, and $\mathbb{B}^{\prime}$ is a $\bmod N^{\prime}$ bi-theta environment, then we shall refer to as a morphism of bi-theta environments $\mathbb{B} \rightarrow \mathbb{B}^{\prime}$ any isomorphism $\mathbb{B}_{N^{\prime}} \stackrel{\sim}{\rightarrow} \mathbb{B}^{\prime}$, where we write $\mathbb{B}_{N^{\prime}}$ for the $\bmod N^{\prime}$ bi-theta environment induced by $\mathbb{B}$.

(iv) In the situation of (iii), if $\ddot{\eta}^{\Theta, l \cdot \underline{\mathbb{Z}} \times \boldsymbol{\mu}_{2}}$ is of standard type, then we shall refer to the resulting $[\bmod N]$ model bi-theta environment as being of standard type.

Proposition 2.14 (Symmetries of Mono-theta and Bi-theta Environments). In the notation of the above discussion: 
(i) The subset

$$
\begin{aligned}
\left(\underline{\underline{\Delta}}_{\underline{\underline{Y}}}^{\mathrm{tp}}\right)^{\Theta}\left[\boldsymbol{\mu}_{N}\right] & \supseteq\left(l \cdot \underline{\Delta}_{\Theta}\right)\left[\boldsymbol{\mu}_{N}\right] \bigcap\left\{\gamma(\beta) \cdot \beta^{-1} \in\left(\underline{\underline{\Delta}}_{\underline{\underline{Y}}}^{\mathrm{tp}}\right)^{\Theta}\left[\boldsymbol{\mu}_{N}\right] \mid \beta \in\left(\underline{\underline{\Delta}}_{\underline{\underline{Y}}}^{\mathrm{tp}}\right)^{\Theta}\left[\boldsymbol{\mu}_{N}\right],\right. \\
\gamma & \in \operatorname{Aut}\left(\underline{\Pi}_{\underline{\underline{Y}}}^{\mathrm{tp}}\left[\boldsymbol{\mu}_{N}\right]\right) \text { s.t. the image of } \gamma \text { in "Out" belongs to } \mathcal{D}_{\underline{\underline{Y}}}, \\
& \text { and } \left.\gamma \text { induces the identity on the quotient } \underline{\underline{\underline{\Pi}}} \underline{\underline{\underline{Y}}}\left[\boldsymbol{\mu}_{N}\right] \rightarrow \underline{\underline{\Pi}}_{\underline{\underline{Y}}}^{\mathrm{tp}} \rightarrow G_{K}\right\}
\end{aligned}
$$

coincides with the image of the tautological section of $\left(l \cdot \underline{\Delta}_{\Theta}\right)\left[\boldsymbol{\mu}_{N}\right] \rightarrow\left(l \cdot \underline{\Delta}_{\Theta}\right)$.

(ii) Let

$$
t_{\underline{\underline{Y}}}^{\Theta}: \underline{\Pi}_{\underline{\underline{Y}}}^{\mathrm{tp}} \rightarrow \underline{\Pi}_{\underline{\underline{Y}}}^{\mathrm{tp}}\left[\boldsymbol{\mu}_{N}\right]
$$

be a section obtained as a conjugate of $s_{\underline{\underline{Y}}}^{\Theta}$, relative to the actions of $K^{\times}$,

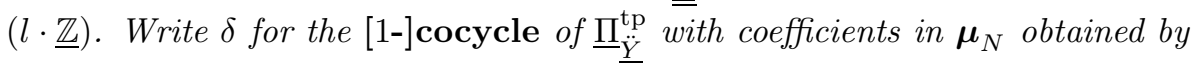
subtracting $s_{\underline{\underline{Y}}}^{\Theta}$ from $t_{\underline{\underline{Y}}}^{\Theta}$ and

$$
\ddot{\alpha}_{\delta} \in \operatorname{Aut}\left(\underline{\underline{\Pi}}_{\underline{\underline{Y}}}^{\mathrm{tp}}\left[\boldsymbol{\mu}_{N}\right]\right)
$$

for the automorphism of the topological group $\underline{\Pi}_{\underline{\underline{Y}}}^{\mathrm{tp}}\left[\boldsymbol{\mu}_{N}\right]$ obtained by "shifting" by $\delta\left[\right.$ which induces the identity on both the quotient $\underline{\Pi}_{\underline{\underline{Y}}}^{\text {tp }}\left[\boldsymbol{\mu}_{N}\right] \rightarrow \underline{\Pi}_{\underline{\underline{Y}}}^{\text {tp }}$ and the kernel of this quotient]. Then $\ddot{\alpha}_{\delta}$ extends to an automorphism $\alpha_{\delta} \overline{\bar{\epsilon}}$ $\operatorname{Aut}\left(\underline{\Pi}_{\underline{\underline{Y}}}^{\mathrm{tp}}\left[\boldsymbol{\mu}_{N}\right]\right)$ which induces the identity on both the quotient $\underline{\Pi}_{\underline{\underline{Y}}}^{\mathrm{tp}}\left[\boldsymbol{\mu}_{N}\right] \rightarrow \underline{\underline{\Pi}}_{\underline{\underline{Y}}}^{\mathrm{tp}}$ and the kernel of this quotient; conjugation by $\alpha_{\delta}$ maps $s_{\underline{\underline{\underline{Y}}}}^{\Theta}$ to $t_{\underline{\underline{\underline{Y}}}}^{\stackrel{\Theta}{=}}$ and preserves the subgroup $\mathcal{D}_{\underline{\underline{Y}}} \subseteq \operatorname{Out}\left(\underline{\underline{\Pi}}_{\underline{\underline{Y}}}^{\mathrm{tp}}\left[\boldsymbol{\mu}_{N}\right]\right)$.

(iii) Write $\mathbb{M} \stackrel{\text { def }}{=}\left(\underline{\Pi}_{\underline{\underline{Y}}}^{\mathrm{tp}}\left[\boldsymbol{\mu}_{N}\right], \mathcal{D}_{\underline{\underline{Y}}}, s_{\underline{\underline{Y}}}^{\stackrel{\Theta}{\underline{Y}}}\right)$ (respectively, $\mathbb{B} \stackrel{\text { def }}{=}\left(\underline{\Pi}_{\underline{\underline{Y}}}^{\mathrm{tp}}\left[\boldsymbol{\mu}_{N}\right], \mathcal{D}_{\underline{\underline{Y}}}, s_{\underline{\underline{Y}}}^{\stackrel{\Theta}{\underline{Y}}}\right.$, $\left.s_{\underline{\underline{Y}}}^{\text {alg }}\right)$ ) for the model mono-theta (respectively, bi-theta) environment constructed in the above discussion. Then every automorphism of $\mathbb{M}$ (respectively, $\mathbb{B})$ determines an automorphism of $\underline{\Pi}_{\underline{\underline{Y}}}^{\mathrm{tp}}[c f$. Proposition $2.11,($ ii $)]$, hence an automorphism of

$$
\underline{\Pi}_{\underline{\underline{X}}}^{\mathrm{tp}}=\operatorname{Aut}\left(\underline{\Pi}_{\underline{\underline{\underline{Y}}}}^{\mathrm{tp}}\right) \times{ }_{\operatorname{Out}\left(\underline{\Pi}_{\underline{\underline{Y}}}^{\mathrm{tp}}\right)} \operatorname{Im}\left(\mathcal{D}_{\underline{\underline{Y}}}\right)
$$

- where $\operatorname{Im}(-)$ " denotes the image in $\operatorname{Out}\left(\underline{\Pi}_{Y}^{\mathrm{tp}}\right)$ - as well as [by considering the cuspidal decomposition groups] an automorphism of the set of cusps of $\underline{\underline{Y}}$. Relative to the labels $\in \mathbb{Z}$ on these cusps $[$ cf. Corollary 2.9 and the discussion preceding $i t]$, this automorphism induces an automorphism $\in(l \cdot \mathbb{Z}) \rtimes\{ \pm 1\}$ of $\mathbb{Z}$. Moreover, the resulting homomorphism

$$
\operatorname{Aut}(\mathbb{M}) \rightarrow(l \cdot \mathbb{Z}) \rtimes\{ \pm 1\}(\text { respectively, } \operatorname{Aut}(\mathbb{B}) \rightarrow(l \cdot \mathbb{Z}) \rtimes\{ \pm 1\})
$$


is surjective (respectively, has image $\operatorname{Im}_{N}$ satisfying $(N \cdot l \cdot \mathbb{Z}) \rtimes\{ \pm 1\} \subseteq \operatorname{Im}_{N} \subseteq$ $\left(N^{\dagger} \cdot l \cdot \mathbb{Z}\right) \rtimes\{ \pm 1\} \subseteq \mathbb{Z} \rtimes\{ \pm 1\}$ - where $N^{\dagger} \stackrel{\text { def }}{=} N$ if $N$ is odd, $N^{\dagger} \stackrel{\text { def }}{=} N / 2$ if $N$ is even).

Proof. First, we consider assertion (i). Observe that the group $\left(\underline{\Delta}_{\underline{\underline{Y}}}^{\mathrm{tp}}\right)^{\Theta}\left[\boldsymbol{\mu}_{N}\right]$ is abelian, and that [since $G_{K}$ is center-free - cf., e.g., [Mzk2], Theorem 1.1.1, (ii)] the automorphisms " $\gamma$ " of $\underline{\underline{\underline{Y}}}_{\underline{\underline{\underline{T}}}}^{\mathrm{tp}}\left[\boldsymbol{\mu}_{N}\right]$ arising from $K^{\times}$restrict to the identity on $\left(\underline{\Delta}_{\underline{\underline{Y}}}^{\mathrm{tp}}\right)^{\Theta}\left[\boldsymbol{\mu}_{N}\right]$. Thus, one computes easily that assertion (i) follows immediately from Proposition 2.12, (ii), in the case where $\Delta_{*}=\underline{\Delta}_{\underline{\underline{X}}}^{\mathrm{tp}}$.

Next, we consider assertion (ii). It is immediate from the definitions that conjugation by $\ddot{\alpha}_{\delta}$ maps $s_{\underline{\underline{\underline{Y}}}}^{\Theta}$ to $t_{\underline{\underline{\underline{Y}}}}^{\Theta}$. Since the outer action of $\operatorname{Gal}(\underline{\underline{Y}} / \underline{\underline{X}})(\cong$ $l \cdot \underline{\mathbb{Z}})$ on $\underline{\underline{\underline{Y}}}_{\underline{\underline{\underline{P}}}}^{\mathrm{tp}}\left[\boldsymbol{\mu}_{N}\right]$ fixes the section $s_{\underline{\underline{\underline{Y}}}}^{\text {alg }}$, up to $\boldsymbol{\mu}_{N^{-}}$-conjugacy, it follows that the difference cocycle $\delta$ determines a cohomology class of

$$
H^{1}\left(\underline{\Pi}_{\underline{\underline{Y}}}^{\mathrm{tp}}, \boldsymbol{\mu}_{N}\right)
$$

that lies in the submodule generated by the Kummer classes of $K^{\times}$and " $\ddot{U}^{2 l \cdot(1 / l)}$ $=\ddot{U}^{2}$ " [cf. Proposition 1.5, (ii), (iii)]. Here, we note that the factor of " $1 / l$ " in the exponent of $\ddot{U}$ arises from the fact that to work with $\ddot{\underline{\eta}}^{\Theta, l \cdot \underline{\mathbb{Z}} \times \boldsymbol{\mu}_{2}}$ amounts to working with $l$-th roots of theta functions [cf. the discussion preceding Definition 2.7]; the factor of "l" arises from the factor of $l$ in " $l \cdot \underline{\mathbb{Z}}$ ".

Since, moreover, the meromorphic function " $\ddot{U}^{2}$ " on $\underline{\underline{\underline{Y}}}$ descends to $\underline{\underline{Y}}$, we thus conclude that $\delta$ extends to a cocycle of $\underline{\Pi}_{\underline{\underline{Y}}}^{\mathrm{tp}}$ with coefficients in $\boldsymbol{\mu}_{N}$, hence that $\ddot{\alpha}_{\delta}$ extends to an automorphism $\alpha_{\delta} \in \overline{\overline{A u t}}\left(\underline{\Pi}_{\underline{\underline{Y}}}^{\mathrm{tp}}\left[\boldsymbol{\mu}_{N}\right]\right)$ which induces the identity on both the quotient $\underline{\Pi}_{\underline{\underline{Y}}}^{\mathrm{tp}}\left[\boldsymbol{\mu}_{N}\right] \rightarrow \underline{\Pi}_{\underline{\underline{Y}}}^{\mathrm{tp}}$ and the kernel of this quotient. Since the action by an element of $\mathrm{Gal}(\underline{\underline{\underline{Y}}} / \underline{\underline{X}})$ clearly maps $\ddot{U}^{2}$ to a $K^{\times}$-multiple of $\ddot{U}^{2}$, it thus follows that conjugation by $\alpha_{\delta}$ preserves $\mathcal{D}_{\underline{\underline{Y}}} \subseteq \operatorname{Out}\left(\underline{\underline{\Pi}}_{\underline{\underline{\underline{p}}}}^{\mathrm{tp}}\left[\boldsymbol{\mu}_{N}\right]\right)[\mathrm{cf}$. the definition of $\left.\mathcal{D}_{\underline{\underline{Y}}} !\right]$, as desired. This completes the proof of assertion (ii).

Finally, assertion (iii) follows immediately from assertion (ii) in the monotheta case by considering [in the context of assertion (ii)] the action of an arbitrary element of $l \cdot \underline{\mathbb{Z}}$. In the bi-theta case, we observe that if, in the situation of assertion (ii), $t_{\underline{\underline{\underline{Y}}}}^{\Theta}$ is obtained as an $N \cdot(l \cdot \underline{\mathbb{Z}})$-conjugate of $s_{\underline{\underline{Y}}}^{\ominus}$, then the cocycle $\delta$ is a coboundary; in particular, [in this case] the automorphism $\alpha_{\delta}$ preserves the $\boldsymbol{\mu}_{N}$-conjugacy classes of subgroups determined by the images of $s_{\underline{\underline{\underline{Y}}}}^{\Theta}, t_{\underline{\underline{\underline{Y}}}}^{\Theta}, s_{\underline{\underline{\underline{Y}}}}^{\text {alg }}$. This shows that $\operatorname{Im}_{N} \supseteq(N \cdot l \cdot \mathbb{Z}) \rtimes\{ \pm 1\}$. On the other hand, the fact that $\operatorname{Im}_{N} \subseteq\left(N^{\dagger} \cdot l \cdot \mathbb{Z}\right) \rtimes\{ \pm 1\}$ follows immediately by considering, in light of the cohomology computation of Proposition 1.5, (i), the third displayed formula 
of Proposition 1.4, (ii), applied to the "mod $N$ étale theta function", which implies [cf. the computation applied in the proof of assertion (ii)] that for any $a \cdot l \in \operatorname{Im}_{N}[$ where $a \in \mathbb{Z}]$, we have $2 a \equiv 0(\bmod N)$.

Remark 2.14.1. Note that, in the notation of Proposition 2.14, (ii), although the automorphism $\ddot{\alpha}_{\delta}$ extends to an automorphism $\alpha_{\delta}$ of $\underline{\Pi}_{\underline{Y}}^{\mathrm{tp}}\left[\boldsymbol{\mu}_{N}\right]$, the automorphism $\alpha_{\delta}$ fails to extend to $\underline{\underline{\Pi}}_{\underline{\underline{X}}}^{\mathrm{tp}}\left[\boldsymbol{\mu}_{N}\right]$ [i.e., since $\ddot{U}^{2}$ fails to descend from $\underline{\underline{Y}}$ to $\underline{\underline{X}} !] ;$ thus, it is essential to work with homomorphisms $s_{\underline{\underline{\underline{Y}}}}^{\Theta}, t_{\underline{\underline{\underline{Y}}}}^{\Theta}: \underline{\Pi}_{\underline{\underline{\underline{Y}}}}^{\mathrm{tp}} \rightarrow$ $\underline{\Pi}_{\underline{\underline{Y}}}^{\mathrm{tp}}\left[\boldsymbol{\mu}_{N}\right]$, as opposed to composites of such homomorphisms with the natural inclusion $\underline{\Pi}_{\underline{\underline{Y}}}^{\mathrm{tp}}\left[\boldsymbol{\mu}_{N}\right] \hookrightarrow \underline{\Pi}_{\underline{\underline{X}}}^{\mathrm{tp}}\left[\boldsymbol{\mu}_{N}\right]$.

Remark 2.14.2. Note that if, in the situation of Proposition 2.14, one tries to replace $\operatorname{Gal}(\underline{\underline{Y}} / \underline{\underline{X}})$ by $\operatorname{Gal}(\underline{\underline{Y}} / \underline{\underline{C}})$, then one must contend with the "inversion automorphism" [cf. Proposition 1.5, (iii)], which maps $\ddot{U} \mapsto \ddot{U}^{-1}$. This obliges one - if one is to retain the property that "conjugation by $\alpha_{\delta}$ preserves $\mathcal{D}_{\underline{\underline{Y}}}$ " - to enlarge " $\mathcal{D}_{\underline{\underline{Y}}}$ " so as to include the outer automorphisms of $\underline{\underline{\Pi}}_{\underline{\underline{Y}}}^{\mathrm{tp}}\left[\boldsymbol{\mu}_{N}\right]$ that arise from Kummer classes of integral powers of $\ddot{U}^{4}=\left(\ddot{U}^{2}\right) \cdot\left(\ddot{U}^{-2}\right)^{-1}$. On the other hand, if one enlarges $\mathcal{D}_{\underline{\underline{Y}}}$ in this fashion, then one verifies easily [cf. the description of the Kummer class of $\ddot{U}$ in Proposition 1.5, (ii)] that the subset considered in Proposition 2.14, (i), is no longer contained in the image of the tautological section of $\left(l \cdot \underline{\Delta}_{\Theta}\right)\left[\boldsymbol{\mu}_{N}\right] \rightarrow\left(l \cdot \underline{\Delta}_{\Theta}\right)$.

Remark 2.14.3. The existence of "shifting automorphisms" as in Proposition 2.14, (ii) — cf. also the mono-theta portion of Proposition 2.14, (iii) may be interpreted as the

\section{"nonexistence of a mono-theta-theoretic basepoint"}

[cf. the discussion preceding Corollary 2.9 concerning "labels"] relative to the $l$. $\underline{\mathbb{Z}}$ action on $\underline{\underline{Y}}$ - i.e., the nonexistence of a "distinguished irreducible component of the special fiber of $\mathfrak{Y}$ " associated to the data constituted by a $\bmod N$ monotheta environment. On the other hand, the description of the poles of the theta function [cf. Proposition 1.4, (i)] already suggests that the data constituted by a $\bmod N$ bi-theta environment [which includes, by considering the "difference" between the subgroups of Definition 2.13, (iii), (c), (d), a choice of a "specific $\bmod N$ étale theta function"] does determine, in effect, a "basepoint modulo $N^{\dagger}$ " [cf. the bi-theta portion of Proposition 2.14, (iii)], i.e., a distinguished irreducible component of the special fiber of $\mathfrak{Y}$, up to the action of $N^{\dagger} \cdot(l \cdot \underline{\mathbb{Z}})$. 
Proposition 2.15 (Discrete Rigidity and $\mathbb{Z}$-torsors). Let us regard $\mathbb{N}_{\geq 1}$ as equipped with the order relation arising from the monoid structure of $\mathbb{N}_{\geq 1}$. [That is to say, for $M, M^{\prime} \in \mathbb{N}_{\geq 1}$, " $M \leq M^{\prime}$ " if and only if $M \mid M^{\prime}$, i.e., $M$ divides $M^{\prime}$.] Write $\mathcal{T}$ for the category whose objects $T_{M}$, where $M \in \mathbb{N}_{\geq 1}$, are copies of $\mathbb{Z}[$ which we think of as torsors over $\mathbb{Z}]$, and whose morphisms $T_{M^{\prime}} \rightarrow T_{M}$, where $M, M^{\prime} \in \mathbb{N}_{\geq 1}$ satisfy $M \mid M^{\prime}$, are the composites of the "identity morphism" $T_{M^{\prime}}=\mathbb{Z} \rightarrow \mathbb{Z}=T_{M}$ with an automorphism of $T_{M}$ arising from the action of an element $\in M \cdot \mathbb{Z} \subseteq \mathbb{Z}$. Let $E \subseteq \mathbb{N}_{\geq 1}$ be a cofinal, totally ordered subset of $\mathbb{N}_{\geq 1}$ such that $1 \in E$. We shall refer to as an $\boldsymbol{E}$-system $\left\{S_{M} ; \beta_{M^{\prime}, M}\right\}_{M, M^{\prime} \in E}$ of $\mathcal{T}$ any projective system

$$
\ldots \longrightarrow S_{M^{\prime}} \stackrel{\beta_{M^{\prime}, M}}{\longrightarrow} S_{M} \longrightarrow \ldots
$$

of objects and morphisms of $\mathcal{T}$ indexed by $E$; we shall refer to as an isomorphism of $\boldsymbol{E}$-systems

$$
\left\{S_{M} ; \beta_{M^{\prime}, M}\right\}_{M, M^{\prime} \in E} \stackrel{\sim}{\rightarrow}\left\{S_{M}^{\prime} ; \beta_{M^{\prime}, M}^{\prime}\right\}_{M, M^{\prime} \in E}
$$

any collection of isomorphisms $\alpha_{M}: S_{M} \stackrel{\sim}{\rightarrow} S_{M}^{\prime}[$ for $M \in E]$ of $\mathcal{T}$ such that $\alpha_{M} \circ \beta_{M^{\prime}, M}=\beta_{M^{\prime}, M}^{\prime} \circ \alpha_{M^{\prime}}\left[\right.$ for $M, M^{\prime} \in E$ such that $\left.M \mid M^{\prime}\right]$. Then:

(i) (Groups of Automorphisms) If $M \in \mathbb{N}_{\geq 1}$, then we have a natural

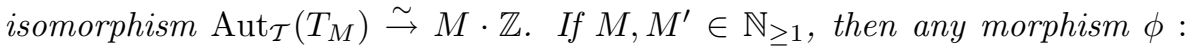
$T_{M^{\prime}} \rightarrow T_{M}$ of $\mathcal{T}$ induces [via the condition of compatibility with $\phi$ ] the injection

$$
\operatorname{Aut}_{\mathcal{T}}\left(T_{M^{\prime}}\right) \stackrel{\sim}{\rightarrow} M^{\prime} \cdot \mathbb{Z} \hookrightarrow M \cdot \mathbb{Z} \stackrel{\sim}{\rightarrow} \operatorname{Aut}_{\mathcal{T}}\left(T_{M}\right)
$$

determined by the natural inclusion $M^{\prime} \cdot \mathbb{Z} \subseteq M \cdot \mathbb{Z}$.

(ii) (Piecewise Rigid $\boldsymbol{E}$-systems) Let $S_{*} \stackrel{\text { def }}{=}\left\{S_{M} ; \beta_{M^{\prime}, M}\right\}_{M, M^{\prime} \in E}, S_{*}^{\prime}$ $\stackrel{\text { def }}{=}\left\{S_{M}^{\prime} ; \beta_{M^{\prime}, M}^{\prime}\right\}_{M, M^{\prime} \in E}$ be arbitrary E-systems such that $S_{M}=S_{M}^{\prime}=T_{1}$ for all $M \in E$. Then there exists an isomorphism of $\boldsymbol{E}$-systems $S_{*} \stackrel{\sim}{\rightarrow} S_{*}^{\prime}$.

(iii) (Piecewise Non-rigid $\boldsymbol{E}$-systems I) For $S_{*} \stackrel{\text { def }}{=}\left\{S_{M} ; \beta_{M^{\prime}, M}\right\}_{M, M^{\prime} \in E}$ an $\boldsymbol{E}$-system such that $S_{M}=T_{M}$ for all $M \in E$, let us write $j_{M}^{S} \stackrel{\text { def }}{=} \beta_{M, 1}(0) \in$ $T_{1}=\mathbb{Z} \subseteq \widehat{\mathbb{Z}}$. Then the sequence $\left\{j_{M}^{S}\right\}_{M \in E}$ converges in $\widehat{\mathbb{Z}}$ to some element, which we denote by $j_{\infty}^{S} \in \widehat{\mathbb{Z}}$. Moreover, the resulting assignment

$$
S_{*} \mapsto j_{\infty}^{S} \in \widehat{\mathbb{Z}}
$$

- where $S_{*}=\left\{S_{M} ; \beta_{M^{\prime}, M}\right\}_{M, M^{\prime} \in E}$ ranges over the E-systems such that $S_{M}=$ $T_{M}$ for all $M \in E-i$ s surjective, and the image of $j_{\infty}^{S}$ in $\widehat{\mathbb{Z}} / \mathbb{Z}$ depends only on the isomorphism class of $S_{*}$ as an E-system. 
(iv) (Piecewise Non-rigid $\boldsymbol{E}$-systems II) There exist non-isomorphic $\boldsymbol{E}$-systems $\left\{S_{M} ; \beta_{M^{\prime}, M}\right\}_{M, M^{\prime} \in E},\left\{S_{M}^{\prime} ; \beta_{M^{\prime}, M}^{\prime}\right\}_{M, M^{\prime} \in E}$ such that $S_{M}=S_{M}^{\prime}=$ $T_{M}$ for all $M \in E$.

Proof. Assertions (i) and (ii) are immediate from the definitions. Next, we consider assertion (iii). The fact that the sequence $\left\{j_{M}^{S}\right\}_{M \in E}$ converges follows immediately from the definitions. To verify the asserted surjectivity, let $j_{\infty} \in \widehat{\mathbb{Z}}$; choose a sequence $\left\{j_{M}\right\}_{M \in E}$ of elements of $\mathbb{Z}$ such that $j_{M}$ maps to the image of $j_{\infty}$ in $(\mathbb{Z} / M \mathbb{Z})$, and $j_{1}=0$. Then for $M, M^{\prime} \in E$ such that $M \mid M^{\prime}$, we take $S_{M} \stackrel{\text { def }}{=} T_{M}, S_{M^{\prime}} \stackrel{\text { def }}{=} T_{M^{\prime}}, \beta_{M^{\prime}, M}$ to be the composite of the "identity morphism" $T_{M^{\prime}}=\mathbb{Z} \rightarrow \mathbb{Z}=T_{M}$ with the automorphism of $T_{M}$ determined by the action of $j_{M^{\prime}}-j_{M} \in M \cdot \mathbb{Z}$ on $T_{M}$. Now it is immediate that the " $j_{\infty}^{S}$ " associated to the resulting $E$-system $S_{*} \stackrel{\text { def }}{=}\left\{S_{M} ; \beta_{M^{\prime}, M}\right\}_{M, M^{\prime} \in E}$ is equal to $j_{\infty}$, as desired. Finally, the fact that isomorphic $E$-systems yield the same element $\in \widehat{\mathbb{Z}} / \mathbb{Z}$ is immediate from the definitions. This completes the proof of assertion (iii). Assertion (iv) follows by taking $S_{*}=\left\{S_{M} ; \beta_{M^{\prime}, M}\right\}_{M, M^{\prime} \in E}$, $S_{*}^{\prime}=\left\{S_{M}^{\prime} ; \beta_{M^{\prime}, M}^{\prime}\right\}_{M, M^{\prime} \in E}$ to be $E$-systems as in assertion (iii) such that the associated " $j_{\infty}^{S}$ ", $j_{\infty}^{S^{\prime} "}$ have distinct images in $\widehat{\mathbb{Z}} / \mathbb{Z}$.

Remark 2.15.1. Proposition 2.15 generalizes immediately to the case of categories equivalent to the category $\mathcal{T}$. We leave the routine details to the reader.

Remark 2.15.2. Let $\mathcal{T}$ be a "connected temperoid" [i.e., the analogue of a Galois category for topological groups such as tempered fundamental groups - cf. [Mzk14], Definition 3.1, (ii)]. For simplicity, we suppose that $\mathcal{T}$ is the temperoid associated to a topological group whose topology admits a countable basis of open subgroups. Then if $A \rightarrow B$ is a morphism of connected Galois objects of $\mathcal{T}$, then one verifies immediately that $\operatorname{Aut}(A)$ acts transitively on $\operatorname{Hom}_{\mathcal{T}}(A, B)$. In particular, [cf. Proposition 2.15, (ii)] if $\mathcal{A} \stackrel{\text { def }}{=}\left\{A_{i}\right\}_{i \in \mathbb{N}}, \mathcal{B} \stackrel{\text { def }}{=}$ $\left\{B_{j}\right\}_{j \in \mathbb{N}}$ are cofinal [i.e., among the connected objects of $\mathcal{T}$ ] projective systems of connected Galois objects of $\mathcal{T}$ indexed by $\mathbb{N}$ [equipped with its usual ordering], then there exists an isomorphism of projective systems $\mathcal{A} \stackrel{\sim}{\rightarrow} \mathcal{B}$ [which does not necessarily induce an isomorphism between the various $\left.A_{i}, B_{j}\right]$. It is this sort of projective system that is implicitly used in the proof of [Mzk14], Proposition 3.2 , to pass from the temperoid to its associated fundamental group.

Corollary 2.16 (Profinite Non-discrete-ness of Bi-theta Environments). Fix some member

$$
\underline{\ddot{\eta}}^{\Theta}
$$


of the collection of [cocycles determined by the collection of] classes $\underline{\underline{\underline{\eta}}}^{\Theta, l \cdot \underline{\mathbb{Z}} \times \boldsymbol{\mu}_{2}}$ [cf. the discussion preceding Proposition 2.14] in $H^{1}\left(\underline{\Pi}_{\ddot{Y}}^{\mathrm{tp}}, l \cdot \underline{\Delta}_{\Theta}\right)$. For $\stackrel{\bar{M}}{\bar{M}} \mathbb{N}_{\geq 1}$, write

$$
\mathbb{B}_{M}
$$

for the model bi-theta environment that arises from [cf. Definition 2.13, (iii)] the reduction of this $\ddot{\eta}^{\Theta}$ modulo $M ; \Pi_{M}\left[\boldsymbol{\mu}_{M}\right] \rightarrow \Pi_{M}$ for the portion of the data $\mathbb{B}_{M}$ constituted $\overline{\bar{b}} y$ the topological group [together with its natural surjection $]$ - cf. Definition 2.13, (iii), (a) $\left[\right.$ so $\Pi_{M}$ may be thought of as a copy of $\left.\underline{\Pi}_{\underline{\underline{Y}}}^{\mathrm{tp}}\right] ; \ddot{\Pi}_{M} \subseteq \Pi_{M}$ for the subgroup which is the image in $\Pi_{M}$ of the theta section - cf. Definition 2.13, (iii), (c) [so $\ddot{\Pi}_{M}$ may be thought of as a copy of $\left.\underline{\Pi}_{\underline{\underline{\underline{Y}}}}^{\mathrm{tp}}\right]$. Let $E \subseteq \mathbb{N}_{\geq 1}$ be a cofinal, totally ordered subset of $\mathbb{N}_{\geq 1}[$ cf. Proposition 2.15] such that $1 \in E$. Thus, we obtain a natural projective system of bi-theta environments

$$
\ldots \longrightarrow \mathbb{B}_{M^{\prime}} \stackrel{\beta_{M^{\prime}, M}}{\longrightarrow} \mathbb{B}_{M} \longrightarrow \ldots
$$

- where $M, M^{\prime} \in E ; M \mid M^{\prime}$. Let $j_{\infty} \in l \cdot \underline{\underline{\mathbb{Z}}}$. Then there exists a projective system of bi-theta environments

$$
\ldots \longrightarrow \mathbb{B}_{M^{\prime}} \stackrel{\gamma_{M^{\prime}, M}}{\longrightarrow} \mathbb{B}_{M} \longrightarrow \ldots
$$

- where $M, M^{\prime} \in E ; M \mid M^{\prime}$ - such that the following properties hold: (a) for each $\gamma_{M^{\prime}, M}$, there exist automorphisms $\alpha, \alpha^{\prime}$ of the bi-theta environments $\mathbb{B}_{M}, \mathbb{B}_{M^{\prime}}$, respectively, [where $\alpha, \alpha^{\prime}$ may depend on the pair $\left.\left(M, M^{\prime}\right)\right]$ such that $\gamma_{M^{\prime}, M}=\alpha \circ \beta_{M^{\prime}, M} \circ \alpha^{\prime} ;(b)$ the classes [indexed by $\left.M\right]$ of

$$
H^{1}\left(\ddot{\Pi}_{1}, l \cdot \underline{\Delta}_{\Theta}\right)=H^{1}\left(\underline{\underline{\Pi}}_{\underline{\underline{\underline{Y}}}}^{\mathrm{tp}}, l \cdot \underline{\Delta}_{\Theta}\right)
$$

obtained by transporting the difference of the algebraic and theta sections of $\mathbb{B}_{M}$ down to $\ddot{\Pi}_{1}$ via the isomorphism $\ddot{\Pi}_{M} \stackrel{\sim}{\rightarrow} \ddot{\Pi}_{1}$ induced by $\gamma_{M, 1}$ converge to the element of $H^{1}\left(\underline{\underline{\Pi}}_{\underline{\underline{Y}}^{\wedge}}, l \cdot \underline{\Delta}_{\Theta}\right)$ [where $\underline{\underline{Y}}^{\wedge}$ is the profinite étale covering determined by $\underline{\underline{\underline{Y}}}-c \bar{f}$. Remark 1.6.4] given by the $j_{\infty}$-conjugate of the restriction of $\underline{\underline{\eta}}^{\Theta}$ to $\underline{\underline{\underline{Y}}}^{\wedge}$.

Proof. In light of the symmetries of bi-theta environments [cf. Proposition 2.14, (iii)], Corollary 2.16 follows immediately from Proposition 2.15, (iii). 
Remark 2.16.1.

(i) Observe that the property discussed in Corollary 2.16 [i.e., Proposition 2.15 , (iii)] is indicative of a fundamental qualitative difference between monoand bi-theta environments. Indeed, if one allows the integer $N \geq 1$ to vary [multiplicatively, i.e., in $\mathbb{N}_{\geq 1}$ ], then the various resulting mono- and bi-theta environments naturally determine projective systems. Moreover, it is natural to think of each of the mod $N$ mono- or bi-theta environments appearing in these projective systems as only being known up to isomorphism [cf. Remarks 5.12.1, 5.12 .2 in $\S 5$ below for more on this point]. From this point of view, Proposition 2.15, (i), (ii), when applied to mono-theta environments [cf. Corollary 2.19, (ii), (iii), below], asserts, in effect, that:

If one works with this projective system of mono-theta environments, then in light of the compatibility of the various [collections of subgroups determined by the image of the] theta sections of the mono-theta environments in the projective system, the various $\bmod N$ étale theta classes determine, in the projective limit, a

$$
\text { single "discrete" } l \cdot \underline{\mathbb{Z}} \text {-torsor }
$$

whose reduction modulo $N$ [i.e., the result of applying a change of structure group via the homomorphism $l \cdot \underline{\mathbb{Z}} \rightarrow l \cdot \underline{\mathbb{Z}} / N \cdot l \cdot \underline{\mathbb{Z}}]$ appears in the $\bmod N$ mono-theta environment.

By contrast, Corollary 2.16 implies that if one tries to carry out such a construction in the case of bi-theta environments, then since the projective system in question gives rise to a "basepoint indeterminacy" [cf. Proposition 2.14, (iii)], for the $\bmod N$ bi-theta environment of the system, given by some group lying between the groups $N \cdot l \cdot \underline{\mathbb{Z}}, N^{\dagger} \cdot l \cdot \underline{\mathbb{Z}}$, the resulting projective limit necessarily leads to a "torsor of possible basepoints" over the "non-discrete" profinite limit group $l \cdot \underline{\mathbb{Z}} \stackrel{\text { def }}{=} l \cdot \underline{\mathbb{Z}} \otimes \widehat{\mathbb{Z}}$. Put another way, the crucial "shifting symmetry" that exists in the case of a mono-theta environment [cf. Proposition 2.14, (ii); Remark 2.14.3] gives rise to a "constant [i.e., independent of $N] l \cdot \underline{\mathbb{Z}}$-indeterminacy", hence implies precisely that, in the mono-theta case, the problem of "finding a common basepoint" for the various $(l \cdot \underline{\mathbb{Z}} / N \cdot l \cdot \underline{\mathbb{Z}})$-torsors that appear in the projective system amounts to the issue of trivializing a torsor over the projective limit

$$
\lim _{N}(l \cdot \underline{\mathbb{Z}} / l \cdot \underline{\mathbb{Z}}) \cong\{0\}
$$

— which remains "discrete" — whereas in the case of a bi-theta environment, 
the corresponding torsor is a torsor over the projective limit

$$
\varliminf_{N}(l \cdot \underline{\mathbb{Z}} / N \cdot l \cdot \underline{\mathbb{Z}}) \cong l \cdot \underline{\widehat{\mathbb{Z}}}
$$

— which is "essentially profinite", hence, in particular, "non-discrete".

(ii) Note that the "non-discreteness" discussed in (i), which arose from the lack of symmetry of a bi-theta environment, by comparison to a mono-theta environment [cf. Proposition 2.14, (iii)], cannot be remedied by, for instance, considering "multi-bi-theta environments" in which instead of considering just one theta section, one considers an entire $(l \cdot \underline{\mathbb{Z}} / N \cdot l \cdot \underline{\mathbb{Z}})$-orbit of theta sections. Indeed, if one considers such orbits, then the resulting projective system requires one to consider [not an $l \cdot \underline{\mathbb{Z}}$-orbit of an étale theta function, but rather] an entire orbit over the non-discrete profinite group

$$
\lim _{N}(l \cdot \underline{\mathbb{Z}} / N \cdot l \cdot \underline{\mathbb{Z}}) \cong l \cdot \underline{\widehat{\mathbb{Z}}}
$$

of étale theta functions - i.e., one must contend with essentially the same "non-discreteness" phenomenon as was discussed in (i).

(iii) The "non-discreteness" phenomenon discussed in (i) may also be formulated from a more "cohomological" point of view, by considering the first derived functor $\mathbb{R}^{1} \leftrightarrows$ lim of the projective limit lim. That is to say, if one considers the exact sequence of projective systems of modules

$$
\{0 \rightarrow N \cdot l \cdot \underline{\mathbb{Z}} \rightarrow l \cdot \underline{\mathbb{Z}} \rightarrow(l \cdot \underline{\mathbb{Z}} / N \cdot l \cdot \underline{\mathbb{Z}}) \rightarrow 0\}_{N \geq 1}
$$

obtained by allowing the integer $N \geq 1$ to vary multiplicatively [and taking the transition morphisms to be the morphisms determined by identifying the various copies of $l \cdot \underline{\mathbb{Z}}$ ], then the [unique nontrivial] connecting homomorphism of the long exact sequence associated to the derived functors of "lim" yields a natural isomorphism

$$
(l \cdot \underline{\widehat{\mathbb{Z}}}) /(l \cdot \underline{\mathbb{Z}}) \stackrel{\sim}{\rightarrow} \mathbb{R}^{1} \lim _{\longleftarrow}\{N \cdot l \cdot \underline{\mathbb{Z}}\}_{N \geq 1}
$$

of the associated " $\mathbb{R}^{1} \varliminf^{\underline{i m}}$ " with the "nonarchimedean solenoid" $(l \cdot \underline{\widehat{\mathbb{Z}}}) /(l \cdot \underline{\mathbb{Z}})$. That is to say, the nonvanishing of this group $(l \cdot \underline{\underline{\mathbb{Z}}}) /(l \cdot \underline{\mathbb{Z}})$ is essentially equivalent to the "non-discreteness" phenomenon discussed in (i).

Remark 2.16.2. Although the present paper is essentially only concerned with the "local theory" of the theta function [i.e., over finite extensions of $\mathbb{Q}_{p}$ ], frequently in applications [cf. [Mzk4], [Mzk5]] it is of interest to develop the 
local theory in such a way that it may be related naturally to the "global theory" [i.e., over number fields]. In such situations, one is typically obligated to contend with some sort of homomorphism of topological groups

$$
\phi: \underline{\underline{\Pi}}_{\underline{\underline{X}}}^{\mathrm{tp}} \rightarrow \underline{\Pi}_{\underline{X}_{F}}
$$

relating the tempered fundamental group of the smooth log curve $\underline{\underline{X}}^{\log }$ [appearing in the theory of the present paper] to the profinite fundamental group of a smooth log curve $\underline{\underline{X}}_{F}^{\log }$ over a number field $F$ such that $\underline{\underline{X}}^{\log }$ is obtained from $\underline{\underline{X}}_{F}^{\mathrm{log}}$ by base-changing to some completion $K=F_{v}$ of $F$ at a finite prime $v$. Moreover, typically, one must assume that $\phi$ is only given up to composition with an inner automorphism [i.e., as an "outer homomorphism"]. Alternatively, one may think of $\phi$ "category-theoretically" via its associated morphism of temperoids [cf. [Mzk14], Proposition 3.2]

$$
\mathcal{T} \rightarrow \mathcal{T}_{F}
$$

- i.e., a functor $\mathcal{T}_{F} \rightarrow \mathcal{T}$ [obtained by associating to a $\underline{\Pi}_{\underline{\underline{X}}}{ }_{F}$-set the $\underline{\Pi}_{\underline{\underline{X}}}^{\text {tp }}$-set determined by composing with $\phi]$, which is typically only determined $\overline{u p}$ to isomorphism. In this situation, connected tempered coverings of $\underline{\underline{X}}^{\log }$ [e.g., a finite étale covering of $\left.\underline{\underline{Y}}^{\mathrm{log}}\right]$, which correspond to open subgroups $H \subseteq \underline{\Pi}_{\underline{X}}^{\mathrm{tp}}$, are subject to an indeterminacy with respect to conjugation by elements of the normalizer

$$
N_{\underline{\Pi}_{\underline{X}}}(\operatorname{Im}(H))
$$

of the image of $H$ in $\underline{\Pi}_{\underline{X}_{F}}$ - i.e., as opposed to just the "weaker" indeterminacy with respect to conjugation by elements of the normalizer $N_{\underline{\underline{\underline{X}}}}^{\text {tp }}(H)$, which arises from working with the topological group $\underline{\Pi}_{\underline{X}}^{\mathrm{tp}}$ up to inner automorphism. In this situation, since the two normalizers in question in fact coincide - i.e., we have

$$
N_{\underline{\underline{\Pi}}_{\underline{\underline{X}}}^{\text {tp }}}(H)=N_{\underline{\underline{\Pi}}_{\underline{\underline{X}}}}(\operatorname{Im}(H))
$$

[by Lemma 2.17, (ii), below; the well-known fact that the absolute Galois group $G_{F_{v}}$ is equal to its own normalizer in the absolute Galois group $G_{F}$ - cf., e.g., [Mzk2], Theorem 1.1.1, (i)] — in the present situation, this state of affairs does not in fact result in any further indeterminacy [by comparison to the strictly local situation]. Moreover, the above equality of normalizers also shows that replacing $\underline{\Pi}_{\underline{\underline{X}}}^{\mathrm{tp}}$ by $H$, for instance, does not result [in the present local/global situation] in any reduction in the indeterminacy to which $H$ is subject. Thus, when $H$ corresponds to a finite étale covering of $\underline{\underline{Y}}^{\log }$, the corresponding 
covering will always be subject to an indeterminacy with respect to the action of some finite index subgroup of $\underline{\mathbb{Z}}$ [an indeterminacy which results in the sort of situation discussed in Remark 2.16.1]. Thus, in summary:

The indeterminacy which results in the phenomena discussed in Remark 2.16.1 may be regarded as the inevitable result of attempting to accommodate simultaneously the "two mutually alien copies of $\mathbb{Z}$ " constituted by the geometric Galois group $\underline{\mathbb{Z}}$ and the arithmetic global base $\mathbb{Z} \subseteq \mathbb{Q} \subseteq F$.

[Here, we remark that the "mutual alienness" of these two copies of $\mathbb{Z}$ arises from the fact that [non-finite] tempered coverings only exist $p$-adically, hence fail to descend to coverings defined over a number field.]

Remark 2.16.3. Relative to the analogy between Galois group actions and differentials [cf. the discussion of [Mzk4]], the equality of the normalizers discussed in Remark 2.16.2 may be thought of as a sort of group-theoretic version of the condition that the map from a finite prime of a number field to the global number field be "unramified".

Lemma 2.17 (Discrete Normalizers). If $G_{1}$ is a subgroup of a group $G_{2}$, then write $N_{G_{2}}\left(G_{1}\right)$ for the normalizer of $G_{1}$ in $G_{2}$. Then:

(i) Let $F$ be a group that contains a normal subgroup of finite index $G \subseteq F$ such that $G$ is a free discrete group of finite rank, $H \subseteq F$ a subgroup such that the group $H \cap G$ is nonabelian. Write $\widehat{F}, \widehat{G}$ for the profinite completions of $F, G$ [so we have a natural inclusion $F \hookrightarrow \widehat{F}]$. Then $N_{\widehat{F}}(H)=$ $N_{F}(H)$.

(ii) Let $\Pi$ be the tempered fundamental group of a hyperbolic orbicurve over a finite extension $K_{\square}$ of $\mathbb{Q}_{p}, H \subseteq \Pi$ an open subgroup. Write $\widehat{\Pi}$ for the profinite completion of $\Pi$. Then $N_{\widehat{\Pi}}(H)=N_{\Pi}(H)$.

Proof. The proof of assertion (i) is similar to [but slightly more involved than] the proof of the case $H=G=F$ discussed in [André], Lemma 3.2.1: By replacing $H$ by $H \bigcap G=H \bigcap \widehat{G}$, we may assume that $H \subseteq G$. Let $\left\{x_{i}\right\}_{i \in I}$ [where $I$ is some index set of cardinality $\geq 2$ ] be a set of generators of $H$, $a \in N_{\widehat{F}}(H)$. Now let us fix two distinct elements $i_{1}, i_{2} \in I$ [so $x_{i_{1}}, x_{i_{2}}$ generate a free subgroup of $G$ of rank 2]. Then there exists a subgroup $J \subseteq G \subseteq F$ of finite index such that $x_{i_{1}}, x_{i_{2}} \in J$, and, moreover, $x_{i_{1}}, x_{i_{2}}$ appear in some collection of free generators of $J$ [cf. [Mzk14], Corollary 1.6, (ii)]. In particular, for each $j=1,2$, the centralizer of $x_{i_{j}}$ in the profinite completion $\widehat{J}$ of $J$ is topologically 
generated by $x_{i_{j}}$ [cf., e.g., [Mzk15], Proposition 1.2, (ii)]. Moreover, since $J$ is of finite index in $F$, it follows that there exists a $b \in F$ such that $a \in b \cdot \widehat{J}(\subseteq \widehat{F})$. In particular, it follows that, for each $j=1,2, b^{-1} a x_{i_{j}} a^{-1} b \in F \bigcap \widehat{J}=J$ is conjugate to $x_{i_{j}} \in J$ in $\widehat{J}$. Now by a classical result of P. Stebe [cf. [LynSch], Proposition 4.9], it follows that $J$ is "conjugacy-separated", hence that for each $j=1,2$, there exists an $a_{j} \in b \cdot J(\subseteq F)$ such that $a x_{i_{j}} a^{-1}=a_{j} x_{i_{j}} a_{j}^{-1}$. Thus, for each $j=1,2, c_{j} \stackrel{\text { def }}{=} a^{-1} a_{j}$ belongs to the centralizer of $x_{i_{j}}$ in $\widehat{J}$, hence is of the form $x_{i_{j}}^{\lambda_{j}}$, for some $\lambda_{j} \in \widehat{\mathbb{Z}}$. But this implies that $c_{2}^{-1} c_{1}=a_{2}^{-1} a_{1} \in F \bigcap \widehat{J}=J$, hence [for instance, by considering the image of $c_{1}, c_{2}$ in the abelianization of $\widehat{J}$ ] that $c_{1}, c_{2} \in J \subseteq F$, so $a \in F$, as desired. Assertion (ii) now follows immediately from assertion (i) by applying assertion (i) to quotients of $\Pi$ by characteristic open subgroups of $\Pi$, which contain finite rank free normal subgroups of finite index.

We are now ready to state the two main results of the present $\S 2$ concerning mono-theta environments.

Corollary 2.18 (Group-theoretic Construction of Mono-theta Environments). Let $N \geq 1$ be an integer; $\underline{\underline{Q}}^{\log }$ a smooth log curve of type $\left(1,(\mathbb{Z} / l \mathbb{Z})^{\Theta}\right)$ over a finite extension $K$ of $\overline{\mathbb{Q}_{p}}$, where l and $p$ are odd, such that $K=\ddot{K} ; \underline{\ddot{\eta}}^{\Theta, l \cdot \underline{\mathbb{Z}} \times \boldsymbol{\mu}_{2}}$ an associated orbit of $l$-th roots of étale theta functions; $\underline{\underline{Y}}^{\log } \rightarrow \underline{\underline{X}}^{\log }, \underline{\underline{Y}}^{\log } \rightarrow \underline{\underline{Y}}^{\log }$ the corresponding coverings [as in the above discus-

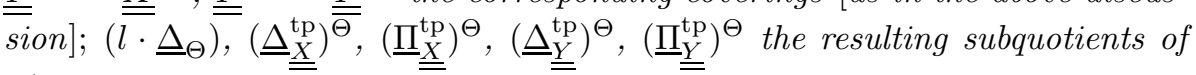
$\underline{\underline{\Pi}}_{\underline{\underline{X}}}^{\mathrm{tp}}[$ as in the above discussion];

$$
\Delta^{\left[\boldsymbol{\mu}_{N}\right]} \stackrel{\text { def }}{=} \operatorname{Ker}\left(\left(l \cdot \underline{\Delta}_{\Theta}\right)\left[\boldsymbol{\mu}_{N}\right] \rightarrow\left(l \cdot \underline{\Delta}_{\Theta}\right)\right)
$$

[i.e., the " $\boldsymbol{\mu}_{N}$ " of " $\left[\boldsymbol{\mu}_{N}\right]$ "];

$$
\underline{\underline{\eta}}^{\Theta, l \cdot \underline{\mathbb{Z}} \times \boldsymbol{\mu}_{2}\left[\boldsymbol{\mu}_{N}\right]}
$$

the collection of classes of $H^{1}\left(\underline{\Pi}_{\underline{Y}}^{\mathrm{tp}}, \Delta^{\left[\boldsymbol{\mu}_{N}\right]}\right)$ obtained by applying the natural surjection $\left(l \cdot \underline{\Delta}_{\Theta}\right) \rightarrow \Delta^{\left[\boldsymbol{\mu}_{N}\right]}$ to $\underline{\underline{\underline{\eta}}}^{\Theta, \underline{\bar{l} \cdot \underline{\underline{Z}}} \times \boldsymbol{\mu}_{2}}$;

$$
\mathcal{D}_{\underline{\underline{Y}}} \subseteq \operatorname{Out}\left(\underline{\Pi}_{\underline{\underline{Y}}}^{\mathrm{tp}}\left[\boldsymbol{\mu}_{N}\right]\right)
$$

the subgroup of $\operatorname{Out}\left(\underline{\Pi}_{\underline{\underline{Y}}}^{\mathrm{tp}}\left[\boldsymbol{\mu}_{N}\right]\right)$ generated by the image of $K^{\times}, \operatorname{Gal}(\underline{\underline{Y}} / \underline{\underline{X}})(\cong l \cdot \underline{\mathbb{Z}})$ [cf. Definition 2.13, $(\overline{\bar{i})}]$;

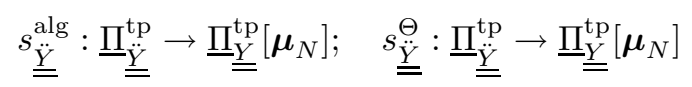


the resulting mod $N$ algebraic and theta sections [determined by a cocycle

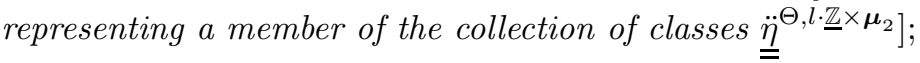

$$
\mathbb{M}_{N} \stackrel{\text { def }}{=}\left(\underline{\Pi}_{\underline{\underline{Y}}}^{\mathrm{tp}}\left[\boldsymbol{\mu}_{N}\right], \mathcal{D}_{\underline{\underline{Y}}}, s_{\underline{\underline{\underline{Y}}}}^{\Theta}\right)
$$

the resulting mod $N$ model mono-theta environment [which, by Proposition 2.14, (ii), is independent, up to isomorphism over the identity of $\underline{\Pi}_{\underline{\underline{Y}}}^{\mathrm{tp}}$, of the choice of [a cocycle representing a member of the collection of classes] $\underline{\ddot{\eta}}^{\Theta, l \cdot \underline{\mathbb{Z}} \times \boldsymbol{\mu}_{2}}$, among its multiples by a 2 -th root of unity]. Then:

(i) (Theta-related Subquotients) Let $\underline{\Pi}_{\underline{X}}^{\bullet}$ be a topological group that is isomorphic to $\underline{\Pi}_{\underline{\underline{X}}}^{\mathrm{t}}$. Then there exists a "functorial group-theoretic algorithm" - i.e., an algorithm that invokes only the structure of $\underline{\Pi}_{\underline{\underline{X}}}$ as an abstract topological group, is functorial with respect to isomorphisms of topological groups, and is devoid of any reference to any isomorphisms of $\underline{\Pi}_{\underline{\underline{X}}}^{*}$ with $\underline{\underline{\Pi}}_{\underline{\underline{X}}}^{\mathrm{tp}}-$ for constructing subquotients

$$
\underline{\underline{\Pi}} \underline{\underline{\underline{Y}}} ; \quad \underline{\Pi}_{\underline{\underline{Y}}}^{\bullet} ; \quad(\underline{\underline{\Pi}} \underline{\underline{\underline{X}}} \rightarrow) G_{K}^{\bullet} ; \quad\left(l \cdot \underline{\Delta}_{\Theta}^{\bullet}\right) ; \quad(\underline{\underline{\Delta}} \underline{\underline{\underline{X}}})^{\Theta} ; \quad\left(\underline{\Pi}_{\underline{\underline{Y}}}^{\bullet}\right)^{\Theta} ; \quad\left(\underline{\underline{\Delta}} \underline{\underline{\underline{Y}}}^{\Theta} ; \quad\left(\underline{\underline{\Pi}}_{\underline{\underline{Y}}}^{\bullet}\right)^{\Theta}\right.
$$

of $\underline{\Pi}_{\underline{\underline{X}}}$, as well as a collection of subgroups of $\underline{\Pi}_{\underline{\underline{X}}}^{\bullet}$ for each element of $(\mathbb{Z} / l \overline{\mathbb{Z}})_{ \pm}$, which have the property that any isomorphism $\underline{\Pi}_{\underline{\underline{X}}} \stackrel{\sim}{\rightarrow} \underline{\Pi}_{\underline{\underline{X}}}^{\text {tp }}$ maps the above subquotients, respectively, to the subquotients

$\underline{\underline{\underline{Y}}}_{\underline{\underline{Y}}}^{\mathrm{tp}} ; \quad \underline{\Pi}_{\underline{\underline{\underline{Y}}}}^{\mathrm{tp}} ; \quad\left(\underline{\underline{\Pi}}_{\underline{\underline{X}}}^{\mathrm{tp}} \rightarrow\right) G_{K} ; \quad\left(l \cdot \underline{\Delta}_{\Theta}\right) ; \quad\left(\underline{\underline{\Delta}}_{\underline{\underline{X}}}^{\mathrm{tp}}\right)^{\Theta} ; \quad\left(\underline{\underline{\Pi}}_{\underline{\underline{X}}}^{\mathrm{tp}}\right)^{\Theta} ; \quad\left(\underline{\underline{\underline{Y}}}_{\underline{\underline{Y}}}^{\mathrm{tp}}\right)^{\Theta} ; \quad\left(\underline{\underline{\Pi}}_{\underline{\underline{Y}}}^{\mathrm{tp}}\right)^{\Theta}$ of $\underline{\underline{\underline{X}}} \underline{\underline{\underline{x}}}$, and the above collection of subgroups to the collection of cuspidal decomposition groups of $\underline{\Pi}_{\underline{\underline{X}}}^{\text {tp }}$ determined by the label $\in(\mathbb{Z} / l \mathbb{Z})_{ \pm}[$cf. Corollary 2.9].

(ii) (From Topological Groups to Mono-theta Environments) In the situation of $(i)$, there exists a "functorial group-theoretic algorithm" for constructing a $\bmod N$ mono-theta environment $\mathbb{M}^{\bullet} \stackrel{\text { def }}{=}\left(\Pi^{\bullet}, \mathcal{D}_{\Pi \bullet}, s_{\Pi \bullet}^{\ominus}\right)$, where

$$
\Pi \stackrel{\Pi^{\text {def }}}{=} \underline{\Pi}_{\underline{\underline{Y}}}^{\bullet} \times_{G_{K}^{\bullet}}\left(\left\{\left(l \cdot \underline{\Delta}_{\Theta}\right) \otimes(\mathbb{Z} / N \mathbb{Z})\right\} \rtimes G_{K}^{\bullet}\right)
$$

[cf. Definition 2.10], "up to isomorphism". More precisely, there exists a "functorial group-theoretic algorithm" for constructing a collection of mod $N$ mono-theta environments $\left\{\mathbb{M}_{\iota}^{\bullet}\right\}_{\iota \in I}$, where $\mathbb{M}_{\iota}^{\bullet}=\left(\Pi^{\bullet}, \mathcal{D}_{\Pi} \bullet,\left(s_{\Pi}^{\Theta}\right)_{\iota}\right)$, such that, for $\iota_{1}, \iota_{2} \in I$, there exists an isomorphism $\mathbb{M}_{\iota_{1}}^{\bullet} \stackrel{\sim}{\rightarrow} \mathbb{M}_{\iota_{2}}^{\bullet}$ that induces the identity on the quotient $\Pi^{\bullet} \rightarrow \underline{\underline{\Pi}} \cdot \underline{\underline{Y}}$. 
(iii) (From Mono-theta Environments to Topological Groups) Let $\mathbb{M} \bullet \stackrel{\text { def }}{=}\left(\Pi \bullet, \mathcal{D}_{\Pi} \bullet, s_{\Pi}^{\Theta}\right)$ be a mod $N$ mono-theta environment isomorphic to $\mathbb{M}_{N}$. Then there exists a "functorial group-theoretic algorithm" i.e., an algorithm that invokes only the structure of $\mathbb{M}^{\bullet}$ as an abstract monotheta environment, is functorial with respect to isomorphisms of mono-theta environments, and is devoid of any reference to any isomorphisms of $\mathbb{M} \bullet$ with $\mathbb{M}_{N}$ - for constructing a quotient

\section{$\underline{\Pi}_{\underline{\underline{Y}}}^{\underline{\underline{Y}}}$}

of $\Pi^{\bullet}$ which has the property that any isomorphism $\mathbb{M}^{\bullet} \stackrel{\sim}{\rightarrow} \mathbb{M}_{N}$ maps this quotient, respectively, to the quotient

$$
\underline{\underline{\underline{Y}}}
$$

of $\underline{\Pi}_{\underline{\underline{Y}}}^{\mathrm{tp}}\left[\boldsymbol{\mu}_{N}\right]$. Moreover, any such isomorphism $\mathbb{M} \bullet \stackrel{\sim}{\rightarrow} \mathbb{M}_{N}$ also induces an isomorphism of

$$
\underline{\Pi}_{\underline{\underline{X}}}^{\bullet} \stackrel{\text { def }}{=} \operatorname{Aut}\left(\underline{\Pi}_{\underline{\underline{Y}}}^{\bullet}\right) \times \operatorname{Out}\left(\underline{\underline{\Pi}}_{\underline{\underline{*}}}\right) \operatorname{Im}\left(\mathcal{D}_{\Pi} \bullet\right)
$$

- where $\operatorname{Im}(-)$ " denotes the image in $\operatorname{Out}\left(\underline{\Pi}_{\underline{\underline{Y}}}^{\bullet}\right)[$ cf. Proposition 2.11, (ii)]; the topology of $\underline{\Pi}_{\underline{\underline{X}}}^{\bullet}$ is the topology determined by taking

$$
\underline{\Pi}_{\underline{\underline{Y}}}^{\bullet} \stackrel{\sim}{\rightarrow} \operatorname{Aut}\left(\underline{\Pi}_{\underline{\underline{Y}}}^{\bullet}\right) \times \operatorname{Out}\left(\underline{\Pi}_{\underline{\underline{Y}}}\right)\{1\} \subseteq \underline{\Pi}_{\underline{\underline{X}}}^{\bullet}
$$

to be an open subgroup - with $\underline{\Pi}_{\underline{\underline{X}}}^{\mathrm{tp}}$. Finally, $\mathbb{M}^{\bullet}$ is isomorphic to the monotheta environment obtained by applying the algorithm of (ii) to $\underline{\Pi}_{\underline{\underline{X}}}^{\bullet}$, via an isomorphism that induces the identity on $\underline{\Pi}_{\underline{\underline{Y}}}^{\cdot}$.

(iv) (Lifting Isomorphisms) For $\square=\alpha$, $\beta$, let $\mathbb{M}^{\square} \stackrel{\text { def }}{=}\left(\Pi^{\square}, \mathcal{D}_{\Pi \square}, s_{\Pi \square}^{\ominus}\right)$ be a mod $N$ mono-theta environment; $\underline{\Pi}_{\underline{\underline{X}}}^{\square}$ be the topological group " $\underline{\underline{\Pi}}_{\underline{X}}$ " of (iii) [i.e., by taking " $\mathbb{M}^{\bullet}$ " to be $\mathbb{M}^{\square}$ ]. Then the natural map [cf. (iii)]

$$
\operatorname{Isom}^{\mu}\left(\mathbb{M}^{\alpha}, \mathbb{M}^{\beta}\right) \rightarrow \operatorname{Isom}\left(\Pi_{\underline{\underline{X}}}^{\alpha}, \Pi_{\underline{\underline{X}}}^{\beta}\right)
$$

- where the superscripted " $\boldsymbol{\mu}$ " denotes the set of $\boldsymbol{\mu}_{N}$-conjugacy classes of isomorphisms - is surjective with fibers of cardinality 1 (respectively, 2) if $N$ is odd (respectively, even). In particular, for any positive integer $M$ such that $M \mid N$, the mod $M$ mono-theta environment $\mathbb{M}_{M}^{\square}$ determined by $\mathbb{M}^{\square}$ induces a natural homomorphism $\operatorname{Aut}^{\mu}\left(\mathbb{M}^{\square}\right) \rightarrow \operatorname{Aut}^{\mu}\left(\mathbb{M}_{M}^{\square}\right)$ with normal image, whose kernel and cokernel have the same cardinalities $[\leq 2]$, respectively, as the kernel and cokernel of the homomorphism $\operatorname{Hom}(\mathbb{Z} / 2 \mathbb{Z}, \mathbb{Z} / N \mathbb{Z}) \rightarrow$ 
$\operatorname{Hom}(\mathbb{Z} / 2 \mathbb{Z}, \mathbb{Z} / M \mathbb{Z})$ induced by the natural surjection $\mathbb{Z} / N \mathbb{Z} \rightarrow \mathbb{Z} / M \mathbb{Z}[$ hence is a bijection if $N / M$ is odd].

Proof. First, we consider assertion (i). An algorithm for constructing the subquotients

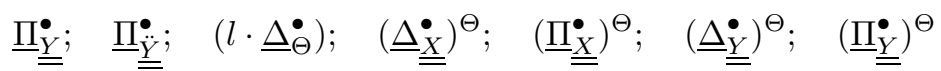

(respectively, $\left.\left(\underline{\Pi}_{X}^{\bullet} \rightarrow\right) G_{K}^{\bullet}\right)$ is described in the proofs of Propositions 1.8, 2.4 [cf. also the de $\overline{\overline{f i n}}$ itions of the various coverings involved!] (respectively, in the proof of [Mzk2], Lemma 1.3.8). An algorithm for constructing the labels of cuspidal decomposition groups is described in the proof of Corollary 2.9 [cf. also the proof of [Mzk2], Lemma 2.3]. This completes the proof of assertion (i). Assertion (ii) follows immediately from the construction of the model monotheta environment in the discussion preceding Definition 2.13; the fact that the various choices involved in this construction yield isomorphic mono-theta environments via isomorphisms that induce the identity on the quotient $\Pi^{\bullet} \rightarrow$ $\underline{\Pi}_{Y}^{\bullet}$ is precisely the content of Proposition 2.14, (ii).

Next, we consider assertion (iii). The algorithm for constructing the quotient $\Pi^{\bullet} \rightarrow \underline{\Pi}_{\underline{Y}}^{\bullet}$ is precisely the content of Proposition 2.11, (ii); the construction of $\underline{\Pi}_{\underline{X}}^{\bullet}$ then follows immediately, in light of the temp-slimness of $\underline{\Pi}_{\underline{X}}^{\bullet}$ [cf. the proof of Proposition 2.11]. The final portion of assertion (iii) [concerning the compatibility with the algorithm of assertion (ii)] follows immediately from the definition of a mono-theta environment [cf. Definition 2.13, (ii)] as "data isomorphic to a model mono-theta environment" [together with the description given in the proof of assertion (ii) of the algorithm of assertion (ii)].

Finally, we consider assertion (iv). First, we observe that the functoriality of the "functorial group-theoretic algorithm" of assertion (iii) yields a natural $\operatorname{map} \operatorname{Isom}^{\mu}\left(\mathbb{M}^{\alpha}, \mathbb{M}^{\beta}\right) \rightarrow \operatorname{Isom}\left(\Pi_{\underline{\underline{X}}}^{\alpha}, \Pi_{\underline{\underline{X}}}^{\beta}\right)$. The surjectivity of this map follows by applying the "functorial group-theoretic algorithm" of assertion (ii), in light of the final portion of assertion (iii) concerning the relation with the algorithm of assertion (ii) [cf., especially, the fact that the isomorphism of mono-theta environments appearing in this final portion induces the identity on " $\underline{\Pi}_{\underline{Y}}^{\bullet}$ "]. The fibers of this map are torsors over [the isomorphic groups] $\operatorname{Ker}\left(\operatorname{Aut}^{\mu}\left(\mathbb{M}^{\square}\right) \rightarrow\right.$ $\operatorname{Aut}\left(\Pi_{\underline{\underline{X}}}^{\square}\right)$ ) [where $\left.\square \in\{\alpha, \beta\}\right]$. To simplify notation, let us set $\mathbb{M}^{\bullet} \stackrel{\text { def }}{=} \mathbb{M}^{\square}$. Next, let us observe that by Corollary 2.19, (i), below [where one checks immediately that there are no "vicious circles" in the reasoning], the natural isomorphism

$$
\left(l \cdot \underline{\Delta}_{\Theta}^{\bullet}\right) \otimes \mathbb{Z} / N \mathbb{Z} \stackrel{\sim}{\rightarrow} \operatorname{Ker}\left(\Pi^{\bullet} \rightarrow \underline{\Pi_{\underline{Y}}^{\bullet}}\right)
$$


is preserved by automorphisms of $\mathbb{M}^{\bullet}$. Thus, $\operatorname{Ker}\left(\operatorname{Aut}^{\mu}\left(\mathbb{M}^{\bullet}\right) \rightarrow \operatorname{Aut}\left(\underline{\Pi}_{\underline{X}}^{\bullet}\right)\right)$ - which consists of automorphisms that act as the identity on $\underline{\underline{\underline{Y}}}$, hence [by applying the above natural isomorphism] also on $\operatorname{Ker}\left(\Pi^{\bullet} \rightarrow \underline{\underline{M}}_{\underline{\underline{Y}}}\right)$ - is naturally isomorphic to the group

$$
\operatorname{Hom}\left(\underline{\Pi}_{\underline{\underline{Y}}}^{\bullet} / \underline{\underline{\Pi}} \underset{\underline{\underline{Y}}}{\bullet}, \operatorname{Ker}\left(\Pi^{\bullet} \rightarrow \underline{\Pi}_{\underline{\underline{Y}}}^{\bullet}\right)\right)
$$

- which is of cardinality 1 (respectively, 2) if $N$ is odd (respectively, even). Moreover, it follows immediately from this description of $\operatorname{Ker}\left(\operatorname{Aut}^{\mu}\left(\mathbb{M}^{\bullet}\right) \rightarrow\right.$ $\left.\operatorname{Aut}\left(\underline{\Pi}_{X}^{\bullet}\right)\right)$ that the natural homomorphism $\operatorname{Aut}^{\mu}\left(\mathbb{M}^{\bullet}\right) \rightarrow \operatorname{Aut}^{\mu}\left(\mathbb{M}_{M}^{\bullet}\right)$ is as described in the statement of assertion (iv). This completes the proof of assertion (iv).

Remark 2.18.1. It follows immediately from Proposition 2.14, (iii), that, for instance, the bijectivity [i.e., "if $N / M$ is odd"] of the latter portion of Corollary 2.18 , (iv), is false for bi-theta environments.

Remark 2.18.2. Thus, in a word, Corollary 2.18 may be interpreted as asserting that a mono-theta environment may be regarded as an object naturally constructed from/associated to the tempered fundamental group. On the other hand, as we shall see in $\S 5$, a mono-theta environment also appears as an object this may be naturally constructed from/associated to a certain Frobenioid. In fact:

One of the main motivating reasons, from the point of view of the author, for the introduction of the notion of a mono-theta environment was precisely the fact that it provides a convenient common ground for relating the [tempered-] étale-theoretic and Frobenioid-theoretic approaches to the theta function.

This point of view will be discussed in more detail in Remark 5.10 .1 in $\S 5$ below.

Corollary 2.19 (Rigidity Properties of Mono-theta Environments). In the notation of Corollary 2.18:

(i) (Cyclotomic Rigidity) Let $\mathbb{M} \bullet \stackrel{\text { def }}{=}\left(\Pi^{\bullet}, \mathcal{D}_{\Pi} \bullet, s_{\Pi}^{\ominus}\right)$ be a $\bmod N$ monotheta environment isomorphic to $\mathbb{M}_{N}$. Thus, by Corollary 2.18, (iii), we obtain a topological group $\underline{\Pi}_{\underline{\underline{X}}}^{\bullet}$ from $\mathbb{M}^{\bullet}$ to which Corollary $2.18,(i)$, (ii), may be applied. Then there exists a "functorial group-theoretic algorithm" i.e., an algorithm that invokes only the structure of $\mathbb{M}^{\bullet}$ as an abstract monotheta environment, is functorial with respect to isomorphisms of mono-theta 
environments, and is devoid of any reference to any isomorphisms of $\mathbb{M}^{\bullet}$ with $\mathbb{M}_{N}$ - for constructing subquotients

$$
\left.\left.\left.\Pi^{\bullet}\right|_{\left(l \cdot \underline{\dot{\theta}}_{\dot{\Theta}}\right)} \subseteq \Pi^{\bullet}\right|_{\left(\underline{\underline{\Delta}}_{\underline{\underline{\dot{Y}}}}\right)^{\Theta}} \subseteq \Pi^{\bullet}\right|_{\left(\underline{\underline{\underline{Y}}}_{\underline{\underline{Y}}}\right)^{\Theta}}
$$

[cf. the notation of Corollary 2.18, (i)] of $\Pi \bullet$ which have the property that any isomorphism $\mathbb{M} \bullet \stackrel{\sim}{\rightarrow} \mathbb{M}_{N}$ maps these subquotients, respectively, to the subquotients

$$
\left(l \cdot \underline{\Delta}_{\Theta}\right)\left[\boldsymbol{\mu}_{N}\right] \subseteq\left(\underline{\underline{\Delta}}_{\underline{\underline{Y}}}^{\mathrm{tp}}\right)^{\Theta}\left[\boldsymbol{\mu}_{N}\right] \subseteq\left(\underline{\underline{\Pi}}_{\underline{\underline{Y}}}^{\mathrm{tp}}\right)^{\Theta}\left[\boldsymbol{\mu}_{N}\right]
$$

of $\underline{\Pi}_{\underline{Y}}^{\mathrm{tp}}\left[\boldsymbol{\mu}_{N}\right]$. Moreover, there exists a "functorial group-theoretic algorithm" for constructing two splittings of the natural surjection

$$
\left.\Pi \cdot\right|_{\left(l \cdot \underline{\bullet}_{\Theta}\right)} \rightarrow\left(l \cdot \underline{\Delta}_{\Theta}^{\bullet}\right)
$$

- hence, in particular, [by forming the difference of these two splittings] an isomorphism of cyclotomes

$$
\left(\left(l \cdot \underline{\Delta}_{\Theta}^{\bullet}\right) \rightarrow\right)(l \cdot \underline{\Delta} \dot{\Theta}) \otimes(\mathbb{Z} / N \mathbb{Z}) \stackrel{\sim}{\rightarrow} \Pi_{\boldsymbol{\mu}}^{\bullet} \stackrel{\text { def }}{=} \operatorname{Ker}\left(\left.\Pi^{\bullet}\right|_{\left(l \cdot \underline{\Delta}_{\Theta}\right)} \rightarrow\left(l \cdot \underline{\Delta}_{\Theta}^{\bullet}\right)\right)
$$

- which have the property that any isomorphism $\mathbb{M} \bullet \stackrel{\sim}{\rightarrow} \mathbb{M}_{N}$ maps these two splittings, respectively, to the two splittings of the surjection

$$
\left(l \cdot \underline{\Delta}_{\Theta}\right)\left[\boldsymbol{\mu}_{N}\right] \rightarrow\left(l \cdot \underline{\Delta}_{\Theta}\right)
$$

determined by the algebraic and theta sections $s_{\ddot{\underline{Y}}}^{\text {alg }}, s_{\ddot{\underline{Y}}}^{\Theta}$ [and hence the above isomorphism of cyclotomes to the natural isomorphism of cyclotomes determined by $s_{\underline{\underline{\underline{Y}}}}^{\text {alg }}, s_{\underline{\underline{\underline{Y}}}}^{\Theta}-c f$. the construction preceding Definition 2.13].

(ii) (Discrete Rigidity) Let $E \subseteq \mathbb{N}_{\geq 1}$ be a cofinal, totally ordered subset of $\mathbb{N}_{\geq 1}[c f$. Proposition 2.15] such that $1 \in E$. Thus, by letting the integer $N$ vary in $E$, we obtain a natural projective system

$$
\ldots \longrightarrow \mathbb{M}_{M^{\prime}} \stackrel{\beta_{M^{\prime}, M}^{*}}{\longrightarrow} \mathbb{M}_{M} \longrightarrow \ldots
$$

of model mono-theta environments indexed by $E$ [cf. Corollary 2.16]. Then any projective system

$$
\ldots \longrightarrow \mathbb{M}_{M^{\prime}}^{\bullet} \stackrel{\gamma_{M^{\prime}, M}^{*}}{\longrightarrow} \mathbb{M}_{M}^{\bullet} \longrightarrow \ldots
$$

- where $M, M^{\prime} \in E ; \mathbb{M}_{M}^{\bullet}$ is a mod $M$ mono-theta environment - is isomorphic to the above natural projective system, i.e., there exists a collection 
of isomorphisms $\alpha_{M}: \mathbb{M}_{M} \stackrel{\sim}{\rightarrow} \mathbb{M}_{M}$ such that $\gamma_{M^{\prime}, M}^{*} \circ \alpha_{M^{\prime}}=\alpha_{M} \circ \beta_{M^{\prime}, M}^{*}$, for $M, M^{\prime} \in E$ satisfying $M \mid M^{\prime}[$ cf. Proposition 2.15, (ii)].

(iii) (Constant Multiple Rigidity) Suppose that $\underline{\underline{\underline{\eta}}}^{\Theta, l \cdot \underline{\mathbb{Z}} \times \boldsymbol{\mu}_{2}}$ is of standard type $[c f$. Definitions $1.9,(i i) ; 2.7]$. Let $\underline{\Pi}_{\underline{\underline{X}}}$ be a topological group that is isomorphic to $\underline{\Pi}_{\underline{\underline{X}}}^{\mathrm{tp}}$. Then there exists a "functorial group-theoretic algorithm" - i.e., an algorithm that invokes only the structure of $\underline{\Pi}_{\underline{\underline{X}}}^{\bullet}$ as an abstract topological group, is functorial with respect to isomorphisms of topological groups, and is devoid of any reference to any isomorphisms of $\underline{\Pi}_{\underline{\underline{X}}}^{\bullet}$ with $\underline{\Pi}_{\underline{\underline{X}}}^{\mathrm{tp}}-$ for constructing a collection of classes of

$$
H^{1}\left(\underline{\Pi}_{\underline{\underline{Y}}}^{\bullet},\left(l \cdot \underline{\Delta}_{\Theta}^{\bullet}\right)\right)
$$

[cf. the notation of Corollary 2.18, (i)] which has the property that any isomorphism $\underline{\Pi}_{\underline{X}}^{\circ} \stackrel{\sim}{\rightarrow} \underline{\Pi}_{\underline{X}}^{\mathrm{tp}}$ maps the above collection of classes to the collection of classes of

$$
H^{1}\left(\underline{\underline{\underline{Y}}},\left(l \cdot \underline{\Delta}_{\Theta}\right)\right)
$$

given by some multiple of the collection of classes $\underline{\underline{\eta}}^{\Theta}, l \cdot \underline{\underline{Z}} \times \boldsymbol{\mu}_{2}$ by an $l$-th root of unity $[$ cf. Corollary 2.8, (i)]. In particular, given any projective system of mono-theta environments

$$
\ldots \longrightarrow \mathbb{M}_{M^{\prime}}^{\bullet} \stackrel{\gamma_{M^{\prime}, M}^{*}}{\longrightarrow} \mathbb{M}_{M}^{\bullet} \longrightarrow \ldots
$$

as in (ii), by taking a compatible system of members of the above collections of classes associated to the [ " $\underline{\Pi}_{X}^{\bullet}$ " arising, as in Corollary 2.18, (iii), from the] $\mathbb{M}_{M}^{\bullet}$, applying the isomorphisms of cyclotomes of $(i)$, and adding the resulting classes to the ["theta"] sections [cf. Definition 2.13, (i), (c)] of each $\mathbb{M}_{M}^{\bullet}$, one obtains a projective system of bi-theta environments

$$
\ldots \longrightarrow \mathbb{B}_{M^{\prime}}^{\bullet} \stackrel{\gamma_{M^{\prime}, M}}{\longrightarrow} \mathbb{B}_{M}^{\bullet} \longrightarrow \ldots
$$

that is isomorphic to some "natural projective system of bi-theta environments" [of standard type]

$$
\ldots \longrightarrow \mathbb{B}_{M^{\prime}} \stackrel{\beta_{M^{\prime}, M}}{\longrightarrow} \mathbb{B}_{M} \longrightarrow \ldots
$$

[i.e., there exist isomorphisms $\alpha_{M}: \mathbb{B}_{M} \stackrel{\sim}{\rightarrow} \mathbb{B}_{M}^{\bullet}$ such that $\gamma_{M^{\prime}, M} \circ \alpha_{M^{\prime}}=$ $\alpha_{M} \circ \beta_{M^{\prime}, M}$, for $M, M^{\prime} \in E$ satisfying $\left.M \mid M^{\prime}\right]$ as in Corollary 2.16 .

Proof. First, we consider assertion (i). Observe that since the theta and algebraic [i.e., "tautological"] sections coincide over $\operatorname{Ker}\left(\underline{\Pi}_{\underline{\underline{Y}}}^{\bullet} \rightarrow\left(\underline{\Pi}_{\underline{\underline{Y}}}^{\bullet}\right)^{\Theta}\right)[\mathrm{cf}$. 
Proposition 1.3], it follows that $\operatorname{Ker}\left(\left.\Pi^{\bullet} \rightarrow \Pi^{\bullet}\right|_{\left(\underline{\Pi}_{\underline{\underline{Y}}}\right.} \Theta\right)$ may be constructed as the image via the theta section [cf. Definition 2.13, (ii), (c)] of $\operatorname{Ker}\left(\underline{\Pi}_{\underline{Y}}^{\bullet} \rightarrow\left(\underline{\Pi}_{\underline{Y}}^{\bullet}\right)^{\Theta}\right)$. The subquotients

$$
\left.\left.\left.\Pi^{\bullet}\right|_{\left(l \cdot \underline{\theta}_{\Theta}\right)} \subseteq \Pi^{\bullet}\right|_{\left(\underline{\underline{\Delta}}_{\underline{\underline{Y}}}\right)^{\Theta}} \subseteq \Pi^{\bullet}\right|_{\left(\underline{\Pi}_{\underline{\underline{Y}}}\right)^{\Theta}}
$$

may then be constructed as the inverse images via the resulting quotient $\left.\Pi^{\bullet}\right|_{\left(\underline{\Pi}_{\underline{\underline{Y}}}\right)^{\Theta}} \rightarrow\left(\underline{\Pi}_{\underline{\underline{Y}}}^{\bullet}\right)^{\Theta}$ of the subquotients $\left(l \cdot \underline{\underline{\Delta}}_{\Theta}^{\bullet}\right) \subseteq\left(\underline{\underline{\Delta}}_{\underline{\underline{Y}}}^{\bullet}\right)^{\Theta} \subseteq\left(\underline{\Pi}_{\underline{\underline{Y}}}^{\bullet}\right)^{\Theta}$ of Corollary $2.18, \overline{\bar{i}})$. The splitting of the natural surjection

$$
\left.\Pi^{\bullet}\right|_{(l \cdot \underline{\dot{\Theta}} \dot{\Theta})} \rightarrow\left(l \cdot \underline{\Delta}_{\Theta}^{\bullet}\right)
$$

corresponding to the theta section may then be obtained directly from the "theta section portion" of the data that constitutes a mono-theta environment [cf. Definition 2.13, (ii), (c)]; the splitting corresponding to the algebraic section [i.e., the "tautological section"] may then be constructed via the algorithm described in Proposition 2.14, (i). This completes the proof of assertion (i). Assertion (ii) follows immediately from Corollary 2.18, (iv). Here, relative to the point of view of Remark 2.16.1, (iii), we note that assertion (ii) may be thought of as a consequence of the fact that [as is easily verified] the " $\mathbb{R}^{1} \mathrm{lim}^{\text {'s" }}$ of the projective system " $\{\operatorname{Hom}(\mathbb{Z} / 2 \mathbb{Z}, \mathbb{Z} / N \mathbb{Z})\}_{N \in E}$ " of Corollary 2.18, (iv), as well as the projective system " $\left\{\boldsymbol{\mu}_{N}\right\}_{N \in E}$ " [cf. the superscripted " $\boldsymbol{\mu}$ 's" of Corollary 2.18, (iv)], vanish.

Finally, we consider assertion (iii). An algorithm for constructing the étale theta function of standard type is described in the proofs of Theorems 1.6, 1.10; Corollary 2.8, (i) [cf. also the proof of [Mzk14], Theorem 6.8, (iii)]. [Here, we recall in passing that this "algorithm" consists essentially of restricting [candidates for] the étale theta function to the decomposition groups of certain torsion points.] The remainder of assertion (iii) follows, in light of the cyclotomic rigidity of assertion (i) and the discrete rigidity of assertion (ii), from the construction of the model bi-theta environment in the discussion preceding Definition 2.13.

Remark 2.19.1. One way to try to eliminate the indeterminacy discussed in Remarks 2.16.1, 2.16.2 is to attempt to work with profinite coverings of $X^{\log }$ that correspond to the covering $\underline{\underline{X}}^{\log } \rightarrow X^{\log }$ for "l infinite". On the other hand, such coverings amount to taking $N$-th roots [for all integers $N \geq 1$ ] of the theta function. In particular, when $N$ is a power of $p$, this has the effect of annihilating the differentials of the curve under consideration. Since the differentials of the curve play an essential role in the proof of the main result 
of [Mzk11], it thus seems unrealistic [at least at the time of writing] to expect to generalize the main result of [Mzk11] [hence also the theory of $\$ 1$, which depends on this result of [Mzk11] in an essential way] so as to apply to such profinite coverings.

Remark 2.19.2. The "cyclotomic rigidity" of Corollary 2.19, (i), is a consequence of the theta section portion of the data that constitutes a mono-theta environment [cf. Definition 2.13, (ii), (c)], together with the subtle property of the commutator $[-,-]$ discussed in Proposition 2.12 [which takes the place of the algebraic section, an object which does not appear in a mono-theta environment]. Note that this subtle property depends in an essential way on the fact that the étale theta class in question determines an isomorphism between the subquotient $\underline{\Delta}_{\Theta}$ of the tempered fundamental group and the cyclotomic coefficients under consideration [cf. Proposition 1.3]. In particular:

This subtle property fails to hold if instead of considering $\stackrel{\ddot{\underline{\eta}}}{=}, l \cdot \underline{\underline{Z}} \times \boldsymbol{\mu}_{2}$ over $\underline{\underline{Y}}^{\log }$ - i.e., the first power of an $l$-th root of the theta function [cf. the discussion preceding Definition 2.7] — one attempts to use some $M$-th power of the $l$-th root of the theta function for $M>1$.

Put another way, if one tries to work with such an $M$-th power, where $M>1$, then one ends up only being able to assert the desired "cyclotomic rigidity" for the submodule $M \cdot \boldsymbol{\mu}_{N} \subseteq \boldsymbol{\mu}_{N}$ [for, say, $N$ divisible by $M$ ]; that is to say, the "remainder" of $\boldsymbol{\mu}_{N}$ is not rigid, but rather subject to an indeterminacy with respect to the action of $\operatorname{Ker}\left((\mathbb{Z} / N \mathbb{Z})^{\times} \rightarrow(\mathbb{Z} /(N / M) \mathbb{Z})^{\times}\right)$. Alternatively, if, instead of working with torsion coefficients [i.e., $\boldsymbol{\mu}_{N}$ ] one works with $\widehat{\mathbb{Z}}$-flat coefficients [e.g., the inverse limit of the $\boldsymbol{\mu}_{N}$, as $N$ ranges over the integers $\geq 1]$, then one may still obtain the [ $\widehat{\mathbb{Z}}$-flat analogue of the] desired "cyclotomic rigidity" property of Corollary 2.19, (i), for $M>1$, but only at the cost of working with "profinite coverings" whose finite subcoverings are "immune" to automorphism indeterminacy, which [cf. Corollary 5.12 and the following remarks in $\S 5$ below] appears to be somewhat unnatural.

Remark 2.19.3. In the context of the projective systems discussed in Corollary 2.19, (ii), (iii), if one writes $\Delta^{\left[\boldsymbol{\mu}_{\infty}\right]}$ for the inverse limit of the $\Delta^{\left[\boldsymbol{\mu}_{N}\right]}$ [as $N$ ranges over the integers $\geq 1$ ], then one may think of the isomorphism

$$
\left(l \cdot \underline{\Delta}_{\Theta}\right) \stackrel{\sim}{\rightarrow} \Delta^{\left[\mu_{\infty}\right]}
$$

arising from the "cyclotomic rigidity" [i.e., the compatible isomorphisms of cyclotomes] of Corollary 2.19, (i), as determining a sort of "integral structure", 
i.e., a sort of "basepoint" corresponding to the first power of the $l$-th root of the theta function, relative to the various $M$-th powers of the $l$-th root of the theta function [cf. Remark 2.19.2] obtained by composing this isomorphism with the map $\Delta^{\left[\mu_{\infty}\right]} \rightarrow \Delta^{\left[\mu_{\infty}\right]}$ on $\Delta^{\left[\mu_{\infty}\right]}$ given by multiplication by $M$. Put another way:

To work in the absence of such a "basepoint" amounts to sacrificing the datum of an intrisically defined "origin", or "fixed reference point", in the system

$$
\ldots \stackrel{M \cdot}{\longrightarrow} \Delta^{\left[\mu_{\infty}\right]} \stackrel{M \cdot}{\longrightarrow} \Delta^{\left[\mu_{\infty}\right]} \stackrel{M \cdot}{\longrightarrow} \Delta^{\left[\mu_{\infty}\right]} \stackrel{M \cdot}{\longrightarrow} \ldots
$$

obtained by multiplication by $M$ on the cyclotome $\Delta^{\left[\mu_{\infty}\right]}$.

Put another way, there is no intrinsic way to distinguish " $\Delta^{\left[\boldsymbol{\mu}_{\infty}\right]}$ " from " $M$. $\Delta^{\left[\mu_{\infty}\right] "}$ - i.e., the distinction between these two objects is entirely a matter of "arbitrary labels" [which are typically implicit in classical discussions of arithmetic geometry - cf. the discussion of the Introduction to the present paper].

Remark 2.19.4. Before proceeding, it is natural to pause and reflect on the topic of precisely what one gains from the discrete and cyclotomic rigidity of Corollary 2.19, (i), (ii). On the one hand, discrete rigidity assures one that, when one works with the projective systems discussed in Corollary 2.19, (ii), (iii), one may restrict to the $\underline{\mathbb{Z}}$-translates of [an $l$-th root of] the theta function without having to worry about confusion with arbitrary $\underline{\mathbb{Z}}$-translates, which are "unnatural". At the level of theta values [cf., e.g., Proposition 1.4, (iii); the labels of Corollary 2.9], this means that one obtains values in $K^{\times}$, as opposed to $\left(K^{\times}\right)^{\wedge}$; in particular, it makes sense to perform [not just multiplication operations, but also] addition operations involving these values in $K^{\times} \subseteq K$, which is not possible with arbitrary elements of $\left(K^{\times}\right)^{\wedge}$. On the other hand, cyclotomic rigidity assures one that one may work with the first power of [an $l$-th root of] the theta function without having to worry that this first power might be "confused with some arbitrary $\lambda$-th power", for $\lambda \in \widehat{\mathbb{Z}}^{\times}$. At the level of theta values [cf., e.g., Proposition 1.4, (iii); the labels of Corollary 2.9], this means that one need not worry about confusion between the "original desired values" in $K^{\times} \subseteq\left(K^{\times}\right)^{\wedge}$ and arbitrary $\lambda$-th powers of such values in $\left(K^{\times}\right)^{\wedge}$, for $\lambda \in \widehat{\mathbb{Z}}^{\times}$- where again it is useful to recall that raising to the $\lambda$-th power on $\left(K^{\times}\right)^{\wedge}\left[\right.$ for $\left.\lambda \in \widehat{\mathbb{Z}}^{\times}\right]$is not a ring homomorphism [i.e., not compatible with addition] unless $\lambda=1$. 
Remark 2.19.5. Recall that in the proof of [Mzk13], Theorem 4.3 [cf. especially the proof of [Mzk2], Lemma 2.5, (ii)], one finds a "group-theoretic algorithm" for constructing a certain natural isomorphism of cyclotomes, between one cyclotome of geometric origin - which, in the situation of Corollaries $2.18,2.19$, essentially amounts to $\left(l \cdot \underline{\Delta}_{\Theta}\right)$ - and one cyclotome of arithmetic origin - which, in the situation of Corollaries 2.18, 2.19, arises from $G_{K}$. If one combines this isomorphism of cyclotomes with the isomorphism of cyclotomes given in Corollary 2.19, (i), the resulting "two-layer isomorphism of cyclotomes structure" is reminiscent of the "Griffiths semi-transversality" of the "crystalline theta object" in the Hodge-Arakelov theory of elliptic curves [cf. [Mzk5], Theorem 2.8], which arises from the "two-layer deformation" that occurs in the consideration of the "crystalline theta object" [i.e., a deformation of the elliptic curve, together with a deformation of an ample line bundle on the deformed elliptic curve].

\section{$\S 3 . \quad$ Tempered Frobenioids}

In the present $\S 3$, we construct certain Frobenioids [cf. the theory of [Mzk17], [Mzk18]] arising from the geometry of line bundles on tempered coverings of a p-adic curve. After discussing various basic properties of these "tempered Frobenioids" [cf. Theorem 3.7; Corollary 3.8], we explain how certain aspects of the theory of the étale theta function discussed in $\S 1, \S 2$ may be interpreted from the point of view of tempered Frobenioids [cf. Example 3.9].

Let $L$ be a finite extension of $\mathbb{Q}_{p}$ [where $p$ is a prime number], with ring of integers $\mathcal{O}_{L}$ and residue field $k_{L} ; \mathfrak{T}$ the formal scheme given by the $p$-adic completion of $\operatorname{Spec}\left(\mathcal{O}_{L}\right) ; \mathfrak{T}^{\log }$ the formal $\log$ scheme obtained by equipping $\mathfrak{T}$ with the $\log$ structure determined by the unique closed point of $\operatorname{Spec}\left(\mathcal{O}_{L}\right) ; \mathfrak{Z}^{\log }$ a stable log curve over $\mathfrak{T}^{\log }$. Also, we assume that the special fiber $\mathfrak{Z}_{k_{L}}$ of $\mathfrak{Z}$ is split, and that the generic fiber of the algebrization of $\mathfrak{Z}^{\log }$ is a smooth log curve. Write $Z^{\log \stackrel{\text { def }}{=}} \mathfrak{Z}^{\log } \times_{\mathcal{O}_{L}} L$ for the ringed space with $\log$ structure obtained by tensoring the structure sheaf of $\mathfrak{Z}$ over $\mathcal{O}_{L}$ with $L$. In the following discussion, we shall often [by abuse of notation] use the notation $Z^{\log }$ also to denote the generic fiber of the algebrization of $\mathfrak{Z}^{\log }[\mathrm{cf} . \S 1]$.

The universal covering of the dual graph of the special fiber $\mathfrak{Z}_{k_{L}}^{\log }$ of $\mathfrak{Z}^{\log }$ determines an infinite Galois étale covering

$$
\mathfrak{Z}_{\infty}^{\log } \rightarrow \mathfrak{Z}^{\log }
$$

of $\mathfrak{Z}^{\log }$; such "universal combinatorial coverings" appear in the theory of the tempered fundamental group [cf. [André], §4; [Mzk14], Example 3.10]. Thus, 
$\mathfrak{Z}_{\infty}^{\log }$ is a formal log scheme; write $Z_{\infty}^{\log } \stackrel{\text { def }}{=} \mathfrak{Z}_{\infty}^{\log } \times_{\mathcal{O}_{L}}$ L. Also, we shall refer to the inverse image of the divisor of cusps of $\mathfrak{Z}^{\log }$ in $\mathfrak{Z}_{\infty}^{\log }$ as the divisor of cusps of $\mathfrak{Z}_{\infty}^{\log }$ and to $\mathfrak{Z}_{\infty}^{\log }$ as the stable model of $Z_{\infty}^{\log }$.

\section{Definition 3.1.}

(i) A divisor on $\mathfrak{Z}_{\infty}$ whose support lies in the special fiber $\left(\mathfrak{Z}_{\infty}\right)_{k_{L}}$ (respectively, the divisor of cusps of $\mathfrak{Z}_{\infty}^{\log }$; the union of the special fiber and divisor of cusps of $\mathfrak{Z}_{\infty}^{\log }$ ) will be referred to as a non-cuspidal log-divisor (respectively, cuspidal log-divisor; log-divisor) on $\mathfrak{Z}_{\infty}^{\log }$. Write

$$
\operatorname{DIV}\left(\mathfrak{Z}_{\infty}^{\log }\right)\left(\text { respectively, } \operatorname{DIV}_{+}\left(\mathfrak{Z}_{\infty}^{\log }\right) ; \operatorname{Div}\left(\mathfrak{Z}_{\infty}^{\log }\right) ; \operatorname{Div}_{+}\left(\mathfrak{Z}_{\infty}^{\log }\right)\right)
$$

for the monoid of log-divisors (respectively, effective log-divisors; Cartier logdivisors; effective Cartier log-divisors) on $\mathfrak{Z}^{\log }$. Thus, we have natural inclusions

$$
\begin{gathered}
\operatorname{Div}_{+}\left(\mathfrak{Z}_{\infty}^{\log }\right) \subseteq \operatorname{DIV}_{+}\left(\mathfrak{Z}_{\infty}^{\log }\right) \subseteq \operatorname{DIV}\left(\mathfrak{Z}_{\infty}^{\log }\right) \\
\operatorname{Div}_{+}\left(\mathfrak{Z}_{\infty}^{\log }\right) \subseteq \operatorname{Div}\left(\mathfrak{Z}_{\infty}^{\log }\right) \subseteq \operatorname{DIV}\left(\mathfrak{Z}_{\infty}^{\log }\right)
\end{gathered}
$$

and a natural identification $\operatorname{DIV}\left(\mathfrak{Z}_{\infty}^{\log }\right)=\operatorname{DIV}_{+}\left(\mathfrak{Z}_{\infty}^{\log }\right)^{\mathrm{gp}}$.

(ii) A nonzero meromorphic function on $\mathfrak{Z}_{\infty}^{\log }$ whose divisor of zeroes and poles is a log-divisor will be referred to as a log-meromorphic function on $\mathfrak{Z}_{\infty}^{\log }$. The group of $\log$-meromorphic functions on $\mathfrak{Z}_{\infty}^{\log }$ will be denoted Mero $\left(\mathfrak{Z}_{\infty}^{\log }\right)$. A log-meromorphic function arising from $L^{\times}$will be referred to as constant.

Proposition 3.2 (Divisors and Rational Functions on Universal Combinatorial Coverings). In the notation of the above discussion:

(i) There exists a positive integer $n$ such that $n \cdot \operatorname{DIV}_{+}\left(\mathfrak{Z}_{\infty}^{\log }\right) \subseteq \operatorname{Div}_{+}\left(\mathfrak{Z}_{\infty}^{\log }\right)$, $n \cdot \operatorname{DIV}\left(\mathfrak{Z}_{\infty}^{\log }\right) \subseteq \operatorname{Div}\left(\mathfrak{Z}_{\infty}^{\log }\right)$. In particular, there exists a natural isomorphism

$$
\operatorname{Div}_{+}\left(\mathfrak{Z}_{\infty}^{\log }\right)^{\mathrm{pf}} \stackrel{\sim}{\rightarrow} \mathrm{DIV}_{+}\left(\mathfrak{Z}_{\infty}^{\log }\right)^{\mathrm{pf}}
$$

- where $\operatorname{DIV}_{+}\left(\mathfrak{Z}_{\infty}^{\log }\right)^{\mathrm{pf}}$ may be naturally identified with a direct product of copies of $\mathbb{Q}_{\geq 0}$, indexed by the cusps [i.e., irreducible components of the divisor of cusps] and irreducible components of the special fiber of $\mathfrak{Z}_{\infty}^{\log }$.

(ii) The structure morphism $\mathfrak{Z}_{\infty}^{\log } \rightarrow \mathfrak{T}^{\log }$ determines a natural isomorphism $\mathcal{O}_{L} \stackrel{\sim}{\rightarrow} \Gamma\left(\mathfrak{Z}_{\infty}, \mathcal{O}_{\mathfrak{Z}_{\infty}}\right)$ - i.e., "all regular functions on $\mathfrak{Z}_{\infty}$ are constant".

(iii) Let $f$ be a nonzero meromorphic function on $\mathfrak{Z}_{\infty}$ such that for every $N \in \mathbb{N}_{\geq 1}[c f$. $\S 0]$, there exists a meromorphic function $g_{N}$ on $\mathfrak{Z}_{\infty}$ such that $g_{N}^{N}=f$. Then $f=1$. 
Proof. To verify assertion (i), let us first observe that the completion of $\mathfrak{Z}_{\infty}$ along a node of $\mathfrak{Z}_{\infty}$ may be identified with the formal spectrum of a complete local ring of the form $\mathcal{O}_{L}[[x, y]] /\left(x y-\pi_{L}^{e}\right)$, where $\pi_{L}$ is a uniformizer of $\mathcal{O}_{L}$, and $e$ is a positive integer; moreover, despite the "infinite" nature of $\mathfrak{Z}_{\infty}$, the number of "e's" that occur at completions of $\mathfrak{Z}_{\infty}$ along its nodes is finite [cf. the definition of $\mathfrak{Z}_{\infty}^{\log }$ in terms of $\mathfrak{Z}^{\log !}$ !. Now assertion (i) follows from the fact that the two irreducible components of the special fiber of this formal spectrum determine divisors $D, E$ such that $e \cdot D, e \cdot E$ are Cartier [i.e., since they occur as the schematic zero loci of " $x$ ", " $y$ "].

Next, we consider assertion (ii). Let $0 \neq f \in \Gamma\left(\mathfrak{Z}_{\infty}, \mathcal{O}_{\mathfrak{Z}_{\infty}}\right)$; write $V(f)$ for the schematic zero locus of $f$ on $\mathfrak{Z}_{\infty}$. Now observe that for each irreducible component $C$ of $\left(\mathfrak{Z}_{\infty}\right)_{k_{L}}$, there exists an $e_{C} \in \mathbb{Z}_{\geq 0}$ such that the meromorphic function

$$
f \cdot \pi_{L}^{-e_{C}}
$$

- where $\pi_{L}$ is a uniformizer of $\mathcal{O}_{L}$ - has no zeroes or poles at the generic point of $C$. By the discrete structure of $\mathbb{Z}_{\geq 0}$, it follows that there exists an irreducible component $C_{1}$ such that $e_{C_{1}} \leq e_{C}$, for all irreducible components $C$ of $\left(\mathfrak{Z}_{\infty}\right)_{k_{L}}$. Thus, the meromorphic function $f_{1} \stackrel{\text { def }}{=} f \cdot \pi_{L}^{-e_{C_{1}}}$ is regular, i.e., $f_{1} \in$ $\Gamma\left(\mathfrak{Z}_{\infty}, \mathcal{O}_{\mathfrak{Z}_{\infty}}\right)$, and, moreover, has nonzero restriction to $\left(\mathfrak{Z}_{\infty}\right)_{k_{L}}$. On the other hand, since $\left(\mathfrak{Z}_{\infty}\right)_{k_{L}}$ is connected and reduced, and each irreducible component $C$ of $\left(\mathfrak{Z}_{\infty}\right)_{k_{L}}$ is proper and geometrically integral over $k_{L}$ [since we assumed that $\mathfrak{Z}_{k_{L}}$ of $\mathfrak{Z}$ is split], it follows that immediately that the natural morphism $k_{L} \rightarrow \Gamma\left(\left(\mathfrak{Z}_{\infty}\right)_{k_{L}}, \mathcal{O}_{\left(\mathfrak{Z}_{\infty}\right)_{k_{L}}}\right)$ is an isomorphism, hence that $f_{1}=\lambda+\pi_{L} \cdot g$, where $\lambda \in \mathcal{O}_{L}^{\times}, g \in \Gamma\left(\mathfrak{Z}_{\infty}, \mathcal{O}_{\mathfrak{Z}_{\infty}}\right)$. Thus, by repeating this argument [with " $f$ " replaced by " $g$ "] and applying the $p$-adic completeness of $\mathfrak{Z}_{\infty}$, we conclude that $f \in \mathcal{O}_{L}$, as desired.

Finally, we consider assertion (iii). Since $\mathfrak{Z}_{\infty}$ is locally noetherian, it follows immediately from the existence of the $g_{N}$ that the divisor of zeroes and poles of $f$ is 0 , hence, by assertion (ii), that $f$ is a constant $\in \mathcal{O}_{L}^{\times}$. Since $L$ is a finite extension of $\mathbb{Q}_{p}$, it thus follows from the well-known structure of $\mathcal{O}_{L}^{\times}$that

$$
f \in \bigcap_{N \in \mathbb{N} \geq 1}\left(\mathcal{O}_{L}^{\times}\right)^{N}=\{1\}
$$

- i.e., that $f=1$, as desired.

Next, let $K$ be a finite extension of $\mathbb{Q}_{p}$, with ring of integers $\mathcal{O}_{K}$ and residue field $k ; K^{\prime}$ a finite Galois extension of $K$ [cf. Remark 3.3.2 below], with ring of integers $\mathcal{O}_{K^{\prime}}$; $\mathfrak{S}$ the formal stack given by forming the stacktheoretic quotient with respect to the natural action of $\operatorname{Gal}\left(K^{\prime} / K\right)$ of the $p$ - 
adic completion of $\operatorname{Spec}\left(\mathcal{O}_{K^{\prime}}\right)$; $\mathfrak{S}^{\log }$ the formal log stack obtained by equipping $\mathfrak{S}$ with the $\log$ structure determined by the unique closed point of $\operatorname{Spec}\left(\mathcal{O}_{K^{\prime}}\right)$; $\mathfrak{X}^{\log }$ a stable log orbicurve [cf. $\left.\S 0\right]$ over $\mathfrak{S}^{\log }$. Also, we assume that the generic fiber $X^{\log } \stackrel{\text { def }}{=} \mathfrak{X}^{\log } \times \mathcal{O}_{K} K$ [of the algebrization] of $\mathfrak{X}^{\log }$ is a smooth log orbicurve [cf. $§ 0]$. Write

$$
\mathcal{B}^{\text {temp }}\left(X^{\log }\right)
$$

for the temperoid of tempered coverings of $X^{\log }$ [cf. [Mzk14], Example 3.10], $\mathcal{B}(\operatorname{Spec}(K))$ for the Galois category of finite étale coverings of $\operatorname{Spec}(K)$, and

$$
\mathcal{D}_{0} \stackrel{\text { def }}{=} \mathcal{B}^{\text {temp }}\left(X^{\log }\right)^{0} ; \quad \mathcal{D}_{\text {cnst }} \stackrel{\text { def }}{=} \mathcal{B}(\operatorname{Spec}(K))^{0}
$$

- where the superscript "0" denotes the full subcategory constituted by the connected objects [cf. [Mzk17], $\S 0$, for more details]. Thus, if $\underline{\Pi}_{X}^{\text {tp }} \stackrel{\text { def }}{=} \pi_{1}^{\text {temp }}\left(X^{\log }\right)$ is the tempered fundamental group of $X^{\log }$ [cf. [André], §4; [Mzk14], Example 3.10 ], then the temperoid $\mathcal{B}^{\text {temp }}\left(X^{\log }\right)$ is naturally isomorphic [as a temperoid] to the temperoid $\mathcal{B}^{\text {temp }}\left(\underline{\Pi}_{X}^{\mathrm{tp}}\right)$ associated to the tempered group $\underline{\Pi}_{X}^{\mathrm{tp}}[\mathrm{cf} . \S 0]$. In a similar vein, the Galois category $\mathcal{B}(\operatorname{Spec}(K))$ is naturally equivalent to the Galois category $\mathcal{B}\left(G_{K}\right)$ associated to the absolute Galois group $G_{K}$ of $K$. Also, we observe that the natural surjection $\underline{\Pi}_{X}^{\mathrm{tp}} \rightarrow G_{K}$ determines a natural functor $\mathcal{D}_{0} \rightarrow \mathcal{D}_{\text {cnst }}\left[\right.$ cf. [Mzk18], Example 1.3, (ii)]. Write $\underline{\Delta}_{X}^{\text {tp }} \stackrel{\text { def }}{=} \operatorname{Ker}\left(\underline{\Pi}_{X}^{\text {tp }} \rightarrow G_{K}\right)$.

\section{Definition 3.3.}

(i) Let $\Delta$ be a tempered group [cf. $\S 0]$. Then we shall refer to as a tempered filter on $\Delta$ a countable collection of characteristic open subgroups of finite index

$$
\Delta^{\text {fil }}=\left\{\Delta_{i}^{\text {fil }}\right\}_{i \in I}
$$

of $\Delta$ such that the following conditions are satisfied:

(a) We have: $\bigcap_{i \in I} \Delta_{i}^{\text {fil }}=\{1\}$.

(b) Every $\Delta_{i}^{\text {fil }}$ admits a minimal co-free subgroup [cf. §0] $\Delta_{i}^{\text {fil, } \infty}[$ which is necessarily characteristic as a subgroup of $\Delta]$.

(c) For each open subgroup $H \subseteq \Delta$, there exists a [necessarily unique] $i_{H} \in I$ such that $\Delta_{i_{H}}^{\text {fil, }} \subseteq H$, and, moreover, for every $i \in I, \Delta_{i}^{\text {fil, }, \infty} \subseteq$ $H$ implies $\Delta_{i}^{\text {fil, } \infty} \subseteq \Delta_{i_{H}}^{\text {fil, }}$.

In the situation of (c), we shall refer to $\Delta_{i_{H}}^{\text {fil, }}$ as the $\Delta^{\text {fil }}$-closure of $H$ in $\Delta$.

(ii) We shall refer to a tempered filter on $\Delta_{X}^{\text {tp }}$ as a tempered filter on $X^{\log }$. Let

$$
\Delta^{\mathrm{fil}}=\left\{\Delta_{i}^{\mathrm{fil}}\right\}_{i \in I}
$$


be a tempered filter on $X^{\log }$. Suppose that $Z^{\log } \rightarrow X^{\log }$ is a finite étale Galois covering that admits a stable model $\mathfrak{Z}^{\log }$ over the ring of integers of the extension field of $K$ determined by the integral closure of $K$ in $Z^{\log }$ such that the special fiber of $\mathfrak{Z}^{\log }$ is split [i.e., $Z^{\log }$ is a curve as in the discussion at the beginning of the present $\S 3$ ], and, moreover, the open subgroup determined by the [geometric portion of] this covering is equal to one of the $\Delta_{i}^{\text {fil }} \subseteq \Delta_{X}^{\text {tp }}$. Write $\mathfrak{Z}_{\infty}^{\log } \rightarrow \mathfrak{Z}^{\log }$ for the "universal combinatorial covering" of $\mathfrak{Z}^{\log }$ and $Z_{\infty}^{\log } \rightarrow Z^{\log }$ for the generic fiber of $\mathfrak{Z}_{\infty}^{\log } \rightarrow \mathfrak{Z}^{\log }$ [so $Z_{\infty}^{\log } \rightarrow Z^{\log }$ corresponds to the subgroup $\Delta_{i}^{\mathrm{fil}, \infty} \subseteq \Delta_{X}^{\mathrm{tp}}-\mathrm{cf}$. [André], Proposition 4.3.1; [André], the proof of Lemma 6.1.1]. Then we shall refer to $Z_{\infty}^{\log } \rightarrow X^{\log }$ as a $\Delta^{\text {fil }}$-covering of $X^{\log }$. If, moreover, $Y^{\log } \rightarrow X^{\log }$ is a connected tempered covering, which determines an open subgroup $H \subseteq \Delta_{X}^{\mathrm{tp}}$, and $\Delta_{i}^{\mathrm{fil}, \infty} \subseteq H$ is the $\Delta^{\mathrm{fil}}$-closure of $H$, then we shall refer to any covering $Z_{\infty}^{\log } \rightarrow Y^{\log }$ whose composite with $Y^{\log } \rightarrow X^{\log }$ is the covering $Z_{\infty}^{\log } \rightarrow X^{\log }$ as a $\Delta^{\text {fil }}$-closure of $Y^{\log } \rightarrow X^{\log }$. [Thus, the geometric portion — but not the base field! — of a $\Delta^{\text {fil }}$-closure of $Y^{\log } \rightarrow X^{\log }$ is uniquely determined up to isomorphism.]

(iii) Let $\Delta^{\text {fil }}=\left\{\Delta_{i}^{\text {fil }}\right\}_{i \in I}$ be a tempered filter on $X^{\log }$. Then for any connected tempered covering $Y^{\log } \rightarrow X^{\log }$, it makes sense to define

$$
\begin{gathered}
\Phi_{0}\left(Y^{\log }\right) \stackrel{\text { def }}{=} \varliminf_{\lim _{\longrightarrow}} Z_{\infty}^{\log } \operatorname{Div}+\left(\mathfrak{Z}_{\infty}^{\log }\right)^{\operatorname{Gal}\left(Z_{\infty}^{\log } / Y^{\log }\right)} \\
\mathbb{B}_{0}\left(Y^{\log }\right) \stackrel{\text { def }}{=} \underline{\lim }_{Z_{\infty}^{\log }} \operatorname{Mero}\left(Z_{\infty}^{\log }\right)^{\operatorname{Gal}\left(Z_{\infty}^{\log } / Y^{\log }\right)}
\end{gathered}
$$

- where the inductive limits range over the $\Delta^{\text {fil }}$-closures $Z_{\infty}^{\log } \rightarrow Y^{\log }$ of $Y^{\log } \rightarrow$ $X^{\log }$; the superscript Galois groups denote the submonoids of elements fixed by the Galois group in question. Moreover, by (i), (c), the assignments $Y^{\log } \mapsto$ $\Phi_{0}\left(Y^{\log }\right), Y^{\log } \mapsto \mathbb{B}_{0}\left(Y^{\log }\right)$ determine functors

$$
\Phi_{0}: \mathcal{D}_{0} \rightarrow \mathfrak{M o n} ; \quad \mathbb{B}_{0}: \mathcal{D}_{0} \rightarrow \mathfrak{M o n}
$$

— where "Mon" is the category of commutative monoids [cf. [Mzk17], §0] together with a natural transformation

$$
\mathbb{B}_{0} \rightarrow \Phi_{0}^{\mathrm{gp}}
$$

[given by assigning to a log-meromorphic function its log-divisor of zeroes and poles], whose image we denote by $\Phi_{0}^{\text {birat }} \subseteq \Phi_{0}^{\mathrm{gp}}$. Also, we shall write $\mathbb{F}_{0} \subseteq \mathbb{B}_{0}$ for the subfunctor determined by the constant log-meromorphic functions and $\Phi_{0}^{\text {cnst }} \subseteq \Phi_{0}^{\mathrm{gp}}$ for the image of $\mathbb{F}_{0}$ in $\Phi_{0}^{\mathrm{gp}}$.

Remark 3.3.1. Note that the set of primes [cf. [Mzk17], §0] of the monoid

$$
\operatorname{Div}_{+}\left(\mathfrak{Z}_{\infty}^{\log }\right)^{\operatorname{Gal}\left(Z_{\infty}^{\log } / Y^{\log }\right)}
$$


appearing in the definition of $\Phi_{0}\left(Y^{\mathrm{log}}\right)$ is in natural bijective correspondence with the set of $\operatorname{Gal}\left(Z_{\infty}^{\log } / Y^{\log }\right)$-orbits of prime log-divisors on $\mathfrak{Z}_{\infty}^{\log }$ [cf. Proposition 3.2, (i)]. Moreover, since, by definition, different $\Delta^{\text {fil }}$-closures $Z_{\infty}^{\log } \rightarrow Y^{\log }$ differ only by an extension of the base field $K$, it follows immediately that in the inductive limit appearing in the definition of $\Phi_{0}\left(Y^{\mathrm{log}}\right)$, the maps between monoids induce isomorphisms of monoids on the respective perfections, hence that the resulting sets of primes map bijectively to one another.

Remark 3.3.2. Note that by taking the extension field $K^{\prime}$ used to define the stack structure of $\mathfrak{S}$ to be "sufficiently large", one may treat the case in which $X^{\log }$ fails to have stable reduction over $\mathcal{O}_{K}$. Moreover, although at first sight the choice of $K^{\prime}$ may appear to be somewhat arbitrary, one verifies immediately that the category $\mathcal{D}_{0}$, as well as the monoids $\Phi_{0}, \mathbb{B}_{0}$ on $\mathcal{D}_{0}$, are unaffected by replacing $K^{\prime}$ by some larger finite Galois extension of $K$.

Proposition 3.4 (Divisor and Rational Function Monoids). In the notation of the above discussion:

(i) $\Phi_{0}\left(Y^{\log }\right)$, as well as each of the monoids

$$
\operatorname{Div}_{+}\left(\mathfrak{Z}_{\infty}^{\log }\right)^{\operatorname{Gal}\left(Z_{\infty}^{\log } / Y^{\log }\right)}
$$

appearing in the inductive limit defining $\Phi_{0}\left(Y^{\log }\right)$, is perf-factorial $[c f$. [Mzk17], Definition 2.4, (i)]. Moreover, every endomorphism of $\Phi_{0}\left(Y^{\log }\right)$ or one of the $\operatorname{Div}_{+}\left(\mathfrak{Z}_{\infty}^{\log }\right)^{\operatorname{Gal}\left(Z_{\infty}^{\log } / Y^{\log }\right)}$ induced by an endomorphism of $Y^{\log }$ over $X^{\log }$ is non-dilating [cf. [Mzk17], Definition 1.1, (i)]. In particular, the functor $\Phi_{0}$ defines a divisorial monoid [cf. [Mzk17], Definition 1.1, (i), (ii)] on $\mathcal{D}_{0}$ which is, moreover, perf-factorial and non-dilating.

(ii) Suppose that $Y^{\log } \rightarrow X^{\log }$ is a connected tempered covering such that the composite morphism $Y^{\log } \rightarrow \operatorname{Spec}(K)$ factors through $\operatorname{Spec}(L)$, for some finite extension $L$ of $K$, in such a way that $Y^{\log }$ is geometrically connected over $L$. Then we have natural isomorphisms of monoids

$$
\begin{gathered}
\mathcal{O}_{L}^{\times} \stackrel{\sim}{\rightarrow} \operatorname{Ker}\left(\mathbb{B}_{0}\left(Y^{\log }\right) \rightarrow \Phi_{0}^{\mathrm{gp}}\left(Y^{\log }\right)\right) \subseteq \mathbb{B}_{0}\left(Y^{\log }\right) \\
\mathcal{O}_{L}^{\triangleright} \stackrel{\sim}{\rightarrow} \mathbb{B}_{0}\left(Y^{\log }\right) \times_{\Phi_{0}^{\mathrm{gp}}\left(Y^{\log }\right)} \Phi_{0}\left(Y^{\log }\right) ; \quad L^{\times} \stackrel{\sim}{\rightarrow} \mathbb{F}_{0}\left(Y^{\log }\right) \subseteq \mathbb{B}_{0}\left(Y^{\log }\right)
\end{gathered}
$$

- where "O을

Proof. First, we consider assertion (i). Let $M$ be one of the monoids under consideration. The fact that $M$ is divisorial is immediate from the definitions. The fact that $M$ is perf-factorial then follows immediately from Proposition 3.2 , (i) [cf. also the description of the primes of $M$ in terms of "orbits of prime 
log-divisors" given in Remark 3.3.1]. Now let $\alpha$ be an endomorphism of $M$ that is induced by an endomorphism of $Y^{\log }$ over $X^{\log }$ such that $\alpha$ induces the identity endomorphism on the set of primes of $M$. Then by considering local functions on $\mathfrak{Z}_{\infty}$ that arise from local functions on $\mathfrak{X}$ and vanish at various primes of $M$, it follows that $\alpha$ is the identity, as desired. This completes the proof of assertion (i). Assertion (ii) follows immediately from Proposition 3.2, (ii) [and the definitions].

Lemma 3.5 (Perfections and Realifications of Perf-factorial Submonoids). Let $P, Q$ be perf-factorial monoids such that: $(a) P$ is a submonoid of $Q$; (b) $P$ is group-saturated [cf. $\S 0]$ in $Q ;(c) \mathbb{R}$ supports $Q$ [cf. [Mzk17], Definition 2.4, (ii)]. Then:

(i) The inclusion $P \hookrightarrow Q$ extends uniquely to inclusions $P^{\mathrm{pf}} \hookrightarrow Q$, $P^{\text {rlf }} \hookrightarrow Q$.

(ii) Relative to the inclusions of $(i), P^{\mathrm{pf}}, P^{\mathrm{rlf}}$ are group-saturated in $Q$.

Proof. Indeed, the portion of assertions (i), (ii) involving " $P$ pf" follows immediately from the definitions. Next, let $\mathfrak{p} \in \operatorname{Prime}(P)$ [where "Prime $(-)$ " is as in [Mzk17], §0]. Since $P$ is perf-factorial, it follows that the "primary component" $P_{\mathfrak{p}}$ associated to $\mathfrak{p}$ is isomorphic to $\mathbb{Z}_{\geq 0}, \mathbb{Q}_{\geq 0}$, or $\mathbb{R}_{\geq 0}$ [cf. [Mzk17], Definition 2.4, (i), (b)]. Since $\mathbb{R}_{\geq 0}$ acts on $Q$ [cf. condition (c)], it thus follows that the natural homomorphism of monoids $P_{\mathfrak{p}} \hookrightarrow P \hookrightarrow Q$ extends [uniquely] to a homomorphism of monoids $P_{\mathfrak{p}}^{\text {rlf }} \hookrightarrow Q$. Next, observe that it follows from the definition of the realification [cf. [Mzk17], Definition 2.4, (i)] that for every $a \in P^{\mathrm{rlf}}$, there exists an $a^{\prime} \in P^{\mathrm{pf}}$ such that $a^{\prime} \geq a$. In particular, it follows that for each $\mathfrak{q} \in \operatorname{Prime}(Q)$, the sum of the images of the various "primary components $a_{\mathfrak{p}} \in P_{\mathfrak{p}}$ of $a$ " [as $\mathfrak{p}$ ranges over the elements of Prime $\left.(P)\right]$ in $Q_{\mathfrak{q}} \cong \mathbb{R}_{\geq 0}$ is bounded above [i.e., by the image in $Q_{\mathfrak{q}} \cong \mathbb{R}_{\geq 0}$ of $a^{\prime}$, which is welldefined since $\left.a^{\prime} \in P^{\mathrm{pf}}\right]$. Thus, this sum converges to an element of $Q_{\mathfrak{q}} \cong \mathbb{R}_{\geq 0}$. Now, letting $\mathfrak{q}$ range over the elements of $\operatorname{Prime}(Q)$, we conclude that we obtain a homomorphism of monoids

$$
P^{\mathrm{rlf}} \rightarrow Q_{\mathrm{factor}}^{\mathrm{pf}}=Q_{\mathrm{factor}}^{\mathrm{rlf}}
$$

[relative to the notation of [Mzk17], Definition 2.4, (i), (c)]. Since, moreover, $Q$ is perf-factorial, it follows from [Mzk17], Definition 2.4, (i), (d) [together with the existence of an $a^{\prime} \in P^{\mathrm{pf}}$ such that $\left.a^{\prime} \geq a\right]$, that this homomorphism factors through $Q$, hence determines a homomorphism of monoids

$$
\phi: P^{\text {rlf }} \rightarrow Q
$$


that is [easily verified to be] uniquely characterized by the property that it $e x$ tends the natural homomorphism of monoids $P^{\mathrm{pf}} \hookrightarrow Q$. Write $\phi^{\mathrm{gp}}:\left(P^{\mathrm{rlf}}\right)^{\mathrm{gp}} \rightarrow$ $Q^{\mathrm{gp}}$ for the induced homomorphism on groupifications. Next, let $a, b \in P^{\text {rlf }}$ be such that $\phi^{\mathrm{gp}}(a-b) \geq 0$ [i.e., $\phi^{\mathrm{gp}}(a-b) \in Q$ ]. Then for any $a^{\prime}, b^{\prime} \in P^{\mathrm{pf}}$ such that $a^{\prime} \geq a, b^{\prime} \leq b$, we obtain that $\phi^{\mathrm{gp}}\left(a^{\prime}-b^{\prime}\right) \geq \phi^{\mathrm{gp}}(a-b) \geq 0$, hence [by the portion of assertion (ii) concerning " $P$ pf"] that $a^{\prime} \geq b^{\prime}$. On the other hand, since $P$ is perf-factorial [cf. [Mzk17], Definition 2.4, (i), (d)], it follows immediately that if $a \nsupseteq b$, then there exist $a^{\prime}, b^{\prime} \in P^{\mathrm{pf}}(A)$ such that $a^{\prime} \geq a$, $b^{\prime} \leq b, a^{\prime} \geq b^{\prime}$. Thus, we conclude that $a \geq b$. In particular, if $\phi^{\mathrm{gp}}(a-b)=0$, then it follows that there exists a $c \in P^{\text {rlf }}[$ i.e., $c \stackrel{\text { def }}{=} a-b]$ such that $\phi(c)=0$. On the other hand, if $c \neq 0$, then [cf. [Mzk17], Definition 2.4, (i), (d)] there exists a $c^{\prime} \in P^{\text {pf }}$ such that $0<c^{\prime} \leq c$, hence that $0 \leq \phi\left(c^{\prime}\right) \leq 0$, so $\phi\left(c^{\prime}\right)=0$, in contradiction to the injectivity of the natural homomorphism of monoids $P^{\text {pf }} \hookrightarrow Q$. Thus, we conclude that $\phi$ is injective. This completes the proof of the portion of assertions (i), (ii) involving " $P$ rlf".

Remark 3.5.1. Observe that it follows immediately from Lemma 3.5, (i), that a nonzero submonoid $P$ of an $\mathbb{R}$-monoprime [cf. [Mzk17], §0] monoid $Q$ is perf-factorial and group-saturated if and only if it is monoprime.

Remark 3.5.2. Note that the injectivity portion of Lemma 3.5, (i), fails to hold if one omits the crucial hypothesis that $P$ is group-saturated in $Q$. Indeed, this may be seen, for instance, by considering an injection $P \stackrel{\text { def }}{=} \mathbb{Z}_{\geq 0} \oplus \mathbb{Z}_{\geq 0} \hookrightarrow$ $Q \stackrel{\text { def }}{=} \mathbb{R}_{\geq 0}$ that sends the elements $(1,0) ;(0,1)$ of $P$ to [nonzero] $\mathbb{Q}$-linearly independent elements of $\mathbb{R}_{\geq 0}$.

Definition 3.6. In the notation of Definition 3.3, (iii):

(i) Let $\Lambda$ be a monoid type. Define $\Phi_{0}^{\Lambda}, \mathbb{B}_{0}^{\Lambda}, \mathbb{F}_{0}^{\Lambda}$ as follows:

$$
\begin{gathered}
\Phi_{0}^{\mathbb{Z}} \stackrel{\text { def }}{=} \Phi_{0} ; \quad \Phi_{0}^{\mathbb{Q}} \stackrel{\text { def }}{=} \Phi_{0}^{\text {pf }} ; \quad \Phi_{0}^{\mathbb{R}} \stackrel{\text { def }}{=} \Phi_{0}^{\text {rlf }} \\
\mathbb{B}_{0}^{\mathbb{Z}} \stackrel{\text { def }}{=} \mathbb{B}_{0} ; \quad \mathbb{B}_{0}^{\mathbb{Q}} \stackrel{\text { def }}{=} \mathbb{B}_{0}^{\text {pf }} ; \quad \mathbb{B}_{0}^{\mathbb{R}} \stackrel{\text { def }}{=} \mathbb{R} \cdot \Phi_{0}^{\text {birat }} \subseteq\left(\Phi_{0}^{\mathbb{R}}\right)^{\mathrm{gp}} \\
\mathbb{F}_{0}^{\mathbb{Z}} \stackrel{\text { def }}{=} \mathbb{F}_{0} ; \quad \mathbb{F}_{0}^{\mathbb{Q}} \stackrel{\text { def }}{=} \mathbb{F}_{0}^{\text {pf }} ; \quad \mathbb{F}_{0}^{\mathbb{R}} \stackrel{\text { def }}{=} \mathbb{R} \cdot \Phi_{0}^{\text {cnst }} \subseteq\left(\Phi_{0}^{\mathbb{R}}\right)^{\text {gp }}
\end{gathered}
$$

— where $\Phi_{0}^{\text {rlf }}$ is as in [Mzk17], Definition 2.4, (i) [cf. Proposition 3.4, (i)].

(ii) Let $\mathcal{D}$ be a connected, totally epimorphic category, equipped with a functor $\mathcal{D} \rightarrow \mathcal{D}_{0}$;

$$
\left.\Phi \subseteq \Phi^{\mathbb{R}-\log } \stackrel{\text { def }}{=} \Phi_{0}^{\mathbb{R}}\right|_{\mathcal{D}}
$$

a group-saturated [i.e., $\Phi(A)$ is group-saturated in $\Phi^{\mathbb{R}-\log }(A), \forall A \in \mathrm{Ob}(\mathcal{D})$ ] subfunctor in monoids which determines a perf-factorial divisorial monoid on 
$\mathcal{D}$ such that the following conditions are satisfied: (a) the [necessarily groupsaturated] submonoid

$$
\left.\Phi^{\text {bs-fld }} \stackrel{\text { def }}{=}\left(\mathbb{R} \cdot \Phi_{0}^{\text {cnst }}\right)\right|_{\mathcal{D}} \times_{\left(\Phi^{\mathbb{R}-\log )}\right) \mathrm{gp}} \Phi \subseteq \Phi^{\mathbb{R}-\log }
$$

on $\mathcal{D}$ is monoprime [cf. [Mzk17], §0]; (b) the image of the resulting homomorphism of group-like monoids on $\mathcal{D}$

$\left.\mathbb{F} \stackrel{\text { def }}{=} \mathbb{F}_{0}^{\Lambda}\right|_{\mathcal{D}} \times\left(\Phi^{\mathbb{R}-\log )}\right)_{\mathrm{gP}} \Phi^{\mathrm{gp}} \rightarrow\left(\Phi^{\mathrm{bs}-\mathrm{fld}}\right)^{\mathrm{gp}}=\left.\left(\mathbb{R} \cdot \Phi_{0}^{\mathrm{cnst}}\right)\right|_{\mathcal{D}} \times\left(\Phi^{\mathbb{R}-\log )}\right)_{\mathrm{gP}} \Phi^{\mathrm{gP}} \subseteq\left(\Phi^{\mathbb{R}-\log }\right)^{\mathrm{gp}}$ determines a subfunctor in nonzero monoids of $\left(\Phi^{\text {bs-fld }}\right)^{g p}$ [i.e., for every $A \in$ $\mathrm{Ob}(\mathcal{D})$, the homomorphism $\mathbb{F}(A) \rightarrow\left(\Phi^{\mathrm{bs}-\mathrm{fld}}\right)^{\mathrm{gp}}(A)$ is nonzero]. [Thus, it follows from these conditions that for every $A \in \operatorname{Ob}(\mathcal{D})$, the image of the homomorphism $\mathbb{F}(A) \rightarrow\left(\Phi^{\mathrm{bs}-\mathrm{fld}}\right)^{\mathrm{gp}}(A)$ contains a nonzero element of $\Phi^{\mathrm{bs}-\mathrm{fld}}(A)$.] Write $\left.\mathbb{B} \stackrel{\text { def }}{=} \mathbb{B}_{0}^{\Lambda}\right|_{\mathcal{D}} \times_{\left(\Phi^{\mathbb{R}-\log )}\right) \mathrm{gp}} \Phi^{\mathrm{gp}} \rightarrow \Phi^{\mathrm{gp}}$. Thus, the data

$$
\left(\mathcal{D}, \Phi, \mathbb{B}, \mathbb{B} \rightarrow \Phi^{\mathrm{gp}}\right)
$$

determines a model Frobenioid

$$
\mathcal{C}
$$

[cf. [Mzk17], Theorem 5.2, (ii)]. We shall refer to a Frobenioid $\mathcal{C}$ obtained in this way as a tempered Frobenioid and to $\Lambda$ as the monoid type of the tempered Frobenioid $\mathcal{C}$. If $\mathcal{C}$ is of rational (respectively, strictly rational) type [a property which is completely determined by $\Phi$ - cf. [Mzk17], Definition 4.5, (ii)], then we shall say that $\Phi$ is rational (respectively, strictly rational).

(iii) If $A \in \operatorname{Ob}(\mathcal{D})$, then we shall say that an element of $\Phi(A)$ is noncuspidal (respectively, cuspidal) if it arises [cf. the inductive limit that appears in the definition of $\Phi_{0}$ ] from a non-cuspidal (respectively, cuspidal) log-divisor; we shall say that a prime $\mathfrak{p}$ of the monoid $\Phi(A)$ is non-cuspidal (respectively, cuspidal) if the primary elements of $\Phi(A)$ that are contained in $\mathfrak{p}$ are noncuspidal (respectively, cuspidal). In the following, we shall write

$$
\begin{aligned}
\Phi(A)^{\mathrm{ncsp}} \subseteq \Phi(A) ; & \Phi(A)^{\mathrm{csp}} \subseteq \Phi(A) \\
\operatorname{Prime}(\Phi(A))^{\mathrm{ncsp}} \subseteq \operatorname{Prime}(\Phi(A)) ; & \operatorname{Prime}(\Phi(A))^{\text {csp }} \subseteq \operatorname{Prime}(\Phi(A))
\end{aligned}
$$

for the submonoids of non-cuspidal and cuspidal elements and the subsets of non-cuspidal and cuspidal primes, respectively. We shall refer to a pre-step of $\mathcal{C}$ as non-cuspidal (respectively, cuspidal) if its zero divisor is non-cuspidal (respectively, cuspidal).

(iv) The data [cf. (ii)]

$$
\left(\mathcal{D}, \Phi^{\mathrm{bs}-\mathrm{fld}}, \mathbb{F}, \mathbb{F} \rightarrow\left(\Phi^{\mathrm{bs}-\mathrm{fld}}\right)^{\mathrm{gp}}\right)
$$


determines a model Frobenioid

$$
\mathcal{C}^{\text {bs-fld }}
$$

[cf. [Mzk17], Theorem 5.2, (ii)]. Moreover, the natural inclusion $\Phi^{\text {bs-fld }}(-) \subseteq$ $\Phi(-)$ determines a natural faithful functor $\mathcal{C}^{\text {bs-fld }} \rightarrow \mathcal{C}$ which may be applied to think of $\mathcal{C}^{\text {bs-fld }}$ as a subcategory of $\mathcal{C}$ [cf. Remark 3.6.3 below]. Note that it follows immediately from the existence of the natural functor $\mathcal{D}_{0} \rightarrow \mathcal{D}_{\text {cnst }}$ that $\mathcal{C}^{\text {bs-fld }}$ is a $p$-adic Frobenioid in the sense of [Mzk18], Example 1.1, (ii). We shall refer to the Frobenioid $\mathcal{C}^{\text {bs-fld }}$ obtained in this way as the base-field-theoretic hull of the tempered Frobenioid $\mathcal{C}$. Also, we shall refer to a morphism of the Frobenioid $\mathcal{C}$ as base-field-theoretic if its zero divisor belongs to $\Phi^{\text {bs-fld }}(-) \subseteq$ $\Phi(-)$.

(v) We shall say that $\Phi$ is cuspidally pure if the following conditions are satisfied: (a) for every non-cuspidal primary element $x \in \Phi(A)$, where $A \in$ $\mathrm{Ob}(\mathcal{D})$, there exists an element $y \in \Phi^{\mathrm{bs}-\mathrm{fld}}(A)$ such that $x \leq y$; (b) we have

$$
\operatorname{Prime}(\Phi(A))=\operatorname{Prime}(\Phi(A))^{\mathrm{ncsp}} \bigcup \operatorname{Prime}(\Phi(A))^{\mathrm{csp}}
$$

for every $A \in \mathrm{Ob}(\mathcal{D})$.

Remark 3.6.1. Note that the group-saturated-ness hypothesis of Definition 3.6, (ii), may be regarded as the condition that "divisors relative to $\Phi$ are effective if and only if they are effective relative to $\Phi^{\mathbb{R}-\log }$, i.e., if and only if they are effective in the usual sense". Alternatively, this hypothesis [together with the perf-factoriality hypothesis of Definition 3.6, (ii)] may be regarded as the analogue in the present "tempered context" of the monoprime-ness hypothesis in [Mzk18], Example 1.1, (ii) — cf. Remark 3.5.1.

Remark 3.6.2. Observe that the base-field-theoretic hull of Definition 3.6, (iv), is itself a tempered Frobenioid, and, moreover, that every $p$-adic Frobenioid may be obtained in this way [cf. Remarks 3.5.1, 3.6.1]. In particular, it follows that "the notion of a p-adic Frobenioid is a special case of the notion of a tempered Frobenioid". Also, we observe in passing that $\Phi^{\text {bs-fld }}$ is always non-dilating and strictly rational.

Remark 3.6.3. It follows immediately from Proposition 3.4, (ii), and the explicit divisorial description of objects and morphisms of a model Frobenioid given in [Mzk17], Theorem 5.2, (i) [cf. also the equivalences of categories of [Mzk17], Definition 1.3, (iii), (d), determined by the operation of taking the zero divisor of a co-angular pre-step] that the objects of the essential image [cf. $\S 0]$ of the natural functor $\mathcal{C}^{\text {bs-fld }} \rightarrow \mathcal{C}$ may be described as the objects of $\mathcal{C}$ that 
may be "linked" to a Frobenius-trivial object via base-field theoretic pre-steps, while the morphisms of the essential image of the natural functor $\mathcal{C}^{\text {bs-fld }} \rightarrow \mathcal{C}$ may be described as the base-field theoretic morphisms of $\mathcal{C}$ between objects of the essential image of $\mathcal{C}^{\text {bs-fld }} \rightarrow \mathcal{C}$. In particular, the natural functor $\mathcal{C}^{\text {bs-fld }} \rightarrow \mathcal{C}$ is isomorphism-full [cf. §0]. Thus, no confusion arises from "identifying" $\mathcal{C}^{\text {bs-fld }}$ with its essential image via the natural functor $\mathcal{C}^{\text {bs-fld }} \rightarrow \mathcal{C}$ in $\mathcal{C}$ [cf. §0].

Remark 3.6.4. If $\Phi, \mathcal{C}$ are as in Definition 3.6, (ii), then it follows from Lemma 3.5 [applied to the submonoid $\Phi \subseteq \Phi^{\mathbb{R}-\log }$ ] that the respective divisor monoids $\Phi^{\mathrm{pf}}, \Phi^{\mathrm{rlf}}$ of $\mathcal{C}^{\mathrm{pf}}, \mathcal{C}^{\text {rlf }}$ also satisfy the conditions of Definition 3.6, (ii). That is to say, the perfection and realification of a tempered Frobenioids are again tempered Frobenioids.

Remark 3.6.5. One verifies immediately that, when applied to a tempered Frobenioid, the operations of perfection and realification [cf. Remark 3.6.4] are compatible with the operation of passing to the associated base-field-theoretic hull of the tempered Frobenioid.

Remark 3.6.6. In the situation of Definition 3.6, (ii), if one supposes further that $\Phi$ is perfect, then condition (a) follows from condition (b) [or, alternatively, from the condition that $\Phi^{\text {bs-fld }}(A)$ be nonzero for each $\left.A \in \operatorname{Ob}(\mathcal{D})\right]$. Indeed, this follows immediately by applying the factorization homomorphism of [Mzk17], Definition 2.4, (i), (c) [cf. also [Mzk17], Definition 2.4, (i), (d)], associated to the perf-factorial monoid $\Phi(A)$.

Now we have the following "tempered analogue" of [Mzk18], Theorem 1.2:

Theorem 3.7 (Basic Properties of Tempered Frobenioids). In the notation of Definition 3.6:

(i) If $\Lambda=\mathbb{Z}$ (respectively, $\Lambda=\mathbb{R})$, then $\mathcal{C}$ is of unit-profinite (respectively, unit-trivial) type. For arbitrary $\Lambda$, the Frobenioid $\mathcal{C}$ is of isotropic, model [hence, in particular, birationally Frobenius-normalized], and subquasi-Frobenius-trivial type, but not of group-like type.

(ii) Suppose $\mathcal{D}$ is of FSMFF-type, and that $\Phi$ is non-dilating. Then $\mathcal{C}$ is of standard type. If, moreover, $\Phi$ is rational [cf. Definition 3.6, (ii)], then $\mathcal{C}$ is of rationally standard type.

(iii) Let $A \in \mathrm{Ob}(\mathcal{C}) ; A_{\mathcal{D}} \stackrel{\text { def }}{=} \operatorname{Base}(A) \in \mathrm{Ob}(\mathcal{D})$. Write $A_{\text {cnst }} \in \mathrm{Ob}\left(\mathcal{D}_{\text {cnst }}\right)$ for the image of $A_{\mathcal{D}}$ in $\mathcal{D}_{\text {cnst }}$ [cf. the discussion preceding Definition 3.3]. Then the natural action of $\operatorname{Aut}_{\mathcal{C}}(A)$ on $\mathcal{O}^{\triangleright}(A)$ and $\mathcal{O}^{\times}(A)$ factors through 
Aut $_{\mathcal{D}_{\text {cnst }}}\left(A_{\text {cnst }}\right)$. If, moreover, $\Lambda \in\{\mathbb{Z}, \mathbb{Q}\}$, then this factorization determines a faithful action of the image of $\operatorname{Aut}_{\mathcal{C}}(A)$ in $\operatorname{Aut}_{\mathcal{D}_{\text {cnst }}}\left(A_{\text {cnst }}\right)$ on $\mathcal{O}^{\triangleright}(A), \mathcal{O}^{\times}(A)$.

(iv) If $\mathcal{D}$ is $\operatorname{slim}[c f$. [Mzk17], $\S 0]$, and $\Lambda \in\{\mathbb{Z}, \mathbb{R}\}$, then $\mathcal{C}$ is also slim.

Proof. First, we consider assertion (i). In light of the definition of $\mathcal{C}$ as a model Frobenioid, it follows from [Mzk17], Theorem 5.2, (ii), that $\mathcal{C}$ is of isotropic and model type; the fact that $\mathcal{C}$ is of sub-quasi-Frobenius-trivial type follows from [Mzk17], Proposition 1.10, (vi). By Proposition 3.4, (ii) (respectively, by the definition of the realification of a Frobenioid — cf. [Mzk17], Proposition 5.3), it follows that if, moreover, $\Lambda=\mathbb{Z}$ (respectively, $\Lambda=\mathbb{R}$ ), then $\mathcal{C}$ is of unit-profinite (respectively, unit-trivial) type; the condition imposed on $\mathbb{F}$ in Definition 3.6, (ii), (b), implies immediately that $\mathcal{C}$ is not of grouplike type. This completes the proof of assertion (i). As for assertion (ii), let us first observe that since $\underline{\Pi}_{X}^{\mathrm{tp}}$ acts trivially on $K^{\times} / \mathcal{O}_{K}^{\times}$, it follows [cf. also the condition imposed on $\mathbb{F}$ in Definition 3.6, (ii), (b)] that every object of $\left(\mathcal{C}^{\text {un-tr }}\right)^{\text {birat }}$ is Frobenius-compact. Thus, assertion (ii) follows immediately from the definitions. Assertion (iii) follows immediately from Proposition 3.4, (ii). Assertion (iv) follows formally from [Mzk17], Proposition 1.13, (iii) [since, by assertion (i) of the present Theorem 3.7, "condition (b)" of loc. cit. is always satisfied by objects of $\mathcal{C}$ ]. This completes the proof of Theorem 3.7 .

Remark 3.7.1. We recall [cf. [Mzk18], §0] in passing that if $\mathcal{D}$ is of weakly indissectible (respectively, strongly dissectible; weakly dissectible) type, then so is $\mathcal{C}$.

Remark 3.7.2. We recall in passing that $\mathcal{D}_{0}$ is slim [cf. [Mzk14], Example 3.10; [Mzk14], Remark 3.4.1] and of FSM-, hence also of FSMFF-, type [cf. [Mzk18], Example 1.3, (i)].

Corollary 3.8 (Preservation of Base-field-theoretic Morphisms and Hulls). Suppose that for $i=1,2, \mathcal{C}_{i}$ is a tempered Frobenioid whose base category $\mathcal{D}_{i}$ is of FSMFF-type, and whose divisor monoid $\Phi_{i}$ is non-dilating. Let

$$
\Psi: \mathcal{C}_{1} \stackrel{\sim}{\rightarrow} \mathcal{C}_{2}
$$

be an equivalence of categories. Then:

(i) Suppose, for $i=1,2$, that the base category $\mathcal{D}_{i}$ of $\mathcal{C}_{i}$ is Frobeniusslim. Then $\Psi$ preserves the base-field-theoretic morphisms.

(ii) Suppose, for $i=1,2$, that the base category $\mathcal{D}_{i}$ of $\mathcal{C}_{i}$ is Div-slim [relative to $\Phi_{i}$ ]. Then $\Psi$ preserves the base-field-theoretic morphisms and 
induces a compatible equivalence

$$
\mathcal{C}_{1}^{\text {bs-fld }} \stackrel{\sim}{\rightarrow} \mathcal{C}_{2}^{\text {bs-fld }}
$$

of the subcategories $\mathcal{C}_{1}^{\text {bs-fld }} \subseteq \mathcal{C}_{1}, \mathcal{C}_{2}^{\text {bs-fld }} \subseteq \mathcal{C}_{2}$ given by the respective base-fieldtheoretic hulls.

(iii) Suppose that $\Psi$ preserves the base-field-theoretic morphisms, and that $\Phi_{1}, \Phi_{2}$ are cuspidally pure. Then $\Psi$ preserves the non-cuspidal and cuspidal pre-steps. If, moreover, $\Phi_{1}, \Phi_{2}$ are rational, then the induced isomorphism of divisor monoids

$$
\Psi^{\Phi}:\left.\left.\Phi_{1}\right|_{\mathcal{C}_{1}} \stackrel{\sim}{\rightarrow} \Phi_{2}\right|_{\mathcal{C}_{2}}
$$

[lying over $\Psi]$ of [Mzk17], Theorem 4.9, preserves non-cuspidal elements and primes, as well as cuspidal elements and primes.

Proof. Indeed, by Theorem 3.7, (i), (ii), $\mathcal{C}_{1}, \mathcal{C}_{2}$ are of standard and isotropic type, but not of group-like type. In particular, by [Mzk17], Theorem 3.4, (ii); [Mzk17], Theorem 4.2, (i), it follows that $\Psi$ preserves pre-steps and primary steps. Moreover, $\Psi$ is compatible with the operation of passing to the perfection [cf. [Mzk17], Theorem 3.4, (iii)].

Next, we consider assertions (i), (ii). By applying [Mzk17], Theorem 3.4, (iv), in the case of assertion (i), and [Mzk17], Theorem 3.4, (ii); [Mzk17], Corollary 4.11, (ii), in the case of assertion (ii), it follows that $\Psi$ preserves the submonoids " $\mathcal{O}^{\triangleright}(-)$ ". Now observe [cf. Proposition 3.4, (ii); the equivalences of categories of [Mzk17], Definition 1.3, (iii), (d), determined by the operation of taking the zero divisor of a co-angular pre-step] that a pre-step of $\mathcal{C}_{i}$ is base-field-theoretic if and only if its image $A \rightarrow B$ in $\mathcal{C}_{i}^{\text {pf }}$ may be written as a [filtered] projective limit in the category $\left(\mathcal{C}_{i}^{\text {pf }}\right)_{B}^{\text {coa-pre }}$ [where "coa-pre" denotes the subcategory determined by the (necessarily co-angular) pre-steps of $\mathcal{C}_{i}^{\text {pf }}$ ] of pre-steps $A^{\prime} \rightarrow B$ that are abstractly equivalent [cf. $\S 0$ ] to an endomorphism that belongs to "Oํ ${ }^{\triangleright}(-)$ ". Thus, $\Psi$ preserves the base-field-theoretic pre-steps. Note, moreover, that $\Psi$ preserves [cf. [Mzk17], Theorem 3.4, (ii), (iii)] the factorization [cf. [Mzk17], Definition 1.3, (iv), (a)] of a morphism of $\mathcal{C}_{i}$ into a composite of a morphism of Frobenius type, a pre-step, and a pullback morphism. Thus, we conclude that $\Psi$ preserves the base-field-theoretic morphisms. This completes the proof of assertion (i). Next, to complete the proof of assertion (ii), let us observe that, under the assumptions of assertion (ii), $\Psi$ preserves [cf. [Mzk17], Theorem 3.4, (iii); [Mzk17], Corollary 4.11, (ii)] the Frobenius-trivial objects. Since $\Psi$ preserves base-field-theoretic pre-steps 
and base-field-theoretic morphisms, it follows from the explicit description of the base-field-theoretic hull given in Remark 3.6.3 that $\Psi$ preserves the [objects and morphisms of the] subcategories $\mathcal{C}_{1}^{\text {bs-fld }} \subseteq \mathcal{C}_{1}, \mathcal{C}_{2}^{\text {bs-fld }} \subseteq \mathcal{C}_{2}$, hence induces an

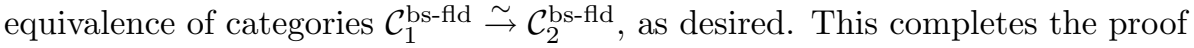
of assertion (ii).

Finally, we consider assertion (iii). Since, by assumption, $\Psi$ preserves the base-field-theoretic pre-steps, we conclude from Definition 3.6, (v), (a) [cf. also the first equivalence of categories involving pre-steps of [Mzk17], Definition 1.3, (iii), (d)], that $\Psi$ preserves the primary non-cuspidal steps, hence, [by Definition $3.6,(\mathrm{v}),(\mathrm{b})]$ that $\Psi$ preserves the primary cuspidal steps. Thus, by considering the "factorization homomorphisms" arising from the fact that $\Phi_{1}, \Phi_{2}$ are perffactorial [cf. [Mzk17], Definition 2.4, (i), (c)] in the context of the perfections of $\mathcal{C}_{1}, \mathcal{C}_{2}$, it follows that $\Psi$ preserves the non-cuspidal and cuspidal pre-steps. The remainder of assertion (iii) now follows immediately from the isomorphism $\Psi^{\Phi}:\left.\left.\Phi_{1}\right|_{\mathcal{C}_{1}} \stackrel{\sim}{\rightarrow} \Phi_{2}\right|_{\mathcal{C}_{2}}$ of [Mzk17], Theorem 4.9 [which is applicable in light of Theorem 3.7, (ii)]. This completes the proof of assertion (iii).

Remark 3.8.1. Note that in the situation of Corollary 3.8, (ii), for suitable base categories [i.e., of the sort that appear in [Mzk18], Theorem 2.4] one may apply to the equivalence of categories $\mathcal{C}_{1}^{\text {bs-fld }} \stackrel{\sim}{\rightarrow} \mathcal{C}_{2}^{\text {bs-fld }}$ induced by $\Psi$ the theory of the category-theoreticity of the Kummer and reciprocity maps, as discussed in [Mzk18], Theorem 2.4.

Remark 3.8.2. In the situation of Corollary 3.8, suppose further that $\Psi$ preserves the base-field-theoretic morphisms, and that $\Phi_{1}, \Phi_{2}$ are cuspidally pure and rational [cf. Corollary 3.8, (iii)]. Then observe that by considering zero divisors of base-field-theoretic pre-steps as in the proof of Corollary 3.8, (i), (ii), it follows that [in the notation of Corollary 3.8], for $C_{1} \in \operatorname{Ob}\left(\mathcal{C}_{1}\right)$, $C_{2} \stackrel{\text { def }}{=} \Psi\left(C_{1}\right)$, non-cuspidal primes $\mathfrak{p}_{1}, \mathfrak{q}_{1}$ of $\Phi\left(C_{1}\right)$ such that $\mathfrak{p}_{1} \mapsto \mathfrak{p}_{2}, \mathfrak{q}_{1} \mapsto \mathfrak{q}_{2}$ $\left[\right.$ where $\left.\mathfrak{p}_{2}, \mathfrak{q}_{2} \in \operatorname{Prime}\left(\Phi\left(C_{2}\right)\right)\right]$, we obtain, for $i=1,2$, natural isomorphisms

$$
\left(\mathbb{R}_{\geq 0} \cong\right) \quad \Phi_{i}\left(C_{i}\right)_{\mathfrak{p}_{i}}^{\text {rlf }} \stackrel{\sim}{\rightarrow} \Phi_{i}\left(C_{i}\right)_{\mathfrak{q}_{i}}^{\text {rlf }} \quad\left(\cong \mathbb{R}_{\geq 0}\right)
$$

[i.e., induced by considering the zero divisors of elements of $\mathcal{O}^{\triangleright}\left(C_{i}\right)$ ] which are compatible with the isomorphism $\Phi_{1}\left(C_{1}\right) \stackrel{\sim}{\rightarrow} \Phi_{2}\left(C_{2}\right)$ induced by $\Psi^{\Phi}$.

Finally, we begin to relate the theory of tempered Frobenioids to the theory of the étale theta function, as discussed in $\S 1, \S 2$ : 


\section{Example 3.9. Theta Functions and Tempered Frobenioids.}

(i) Suppose that $X^{\log }$ is a smooth log orbicurve of the sort defined in Definition 2.5 , (i), (ii) [i.e., one of the following smooth log orbicurves: " $\underline{X}^{\log }$ ",

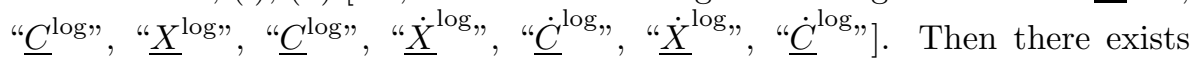
a [1-]commutative diagram of finite log étale Galois coverings of smooth log orbicurves

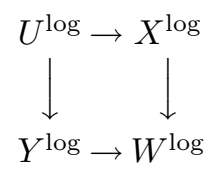

- where $U^{\log }, Y^{\log }$ are smooth log curves that arise as generic fibers of stable log curves $\mathfrak{U}^{\log }, \mathfrak{Y}^{\log }$ over [formal spectra equipped with appropriate log structures determined by] rings of integers of appropriate finite extensions of $K$; the diagram induces a natural isomorphism $\operatorname{Gal}\left(U^{\log } / X^{\log }\right) \stackrel{\sim}{\rightarrow} \operatorname{Gal}\left(Y^{\log } / W^{\log }\right)$; the order of the group $\operatorname{Gal}\left(U^{\log } / X^{\log }\right) \cong \operatorname{Gal}\left(Y^{\log } / W^{\log }\right)$ is $\leq 2 ; Y^{\log } \rightarrow W^{\log }$ is $u n$ ramified at the cusps of $Y^{\log } ; Y^{\log }$ is of genus 1. [Thus, for instance, when $X^{\log }$ is " $\underline{\underline{C}}^{\log , ", ~ o n e ~ m a y ~ t a k e ~ t h e ~ u p p e r ~ a r r o w ~ o f ~ t h e ~ d i a g r a m ~ t o ~ b e ~ " ~} \underline{\underline{X}}^{\log } \rightarrow \underline{\underline{C}}^{\log }$ "

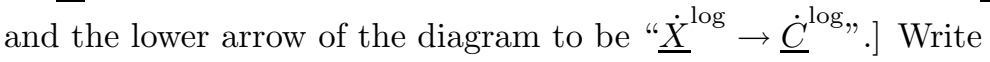

$$
\begin{gathered}
\mathcal{D}_{U} \stackrel{\text { def }}{=} \mathcal{B}^{\text {temp }}\left(U^{\log }\right)^{0} ; \quad \mathcal{D}_{X} \stackrel{\text { def }}{=} \mathcal{B}^{\text {temp }}\left(X^{\log }\right)^{0}\left(=\mathcal{D}_{0}\right) \\
\mathcal{D}_{Y} \stackrel{\text { def }}{=} \mathcal{B}^{\text {temp }}\left(Y^{\log }\right)^{0} ; \quad \mathcal{D}_{W} \stackrel{\text { def }}{=} \mathcal{B}^{\text {temp }}\left(W^{\log }\right)^{0}
\end{gathered}
$$

- so the above [1-]commutative diagram induces natural functors $\mathcal{D}_{U} \rightarrow \mathcal{D}_{X}$, $\mathcal{D}_{U} \rightarrow \mathcal{D}_{Y}, \mathcal{D}_{X} \rightarrow \mathcal{D}_{W}, \mathcal{D}_{Y} \rightarrow \mathcal{D}_{W}$ [obtained by regarding a tempered covering of the domain orbicurve of an arrow of the above commutative diagram as a tempered covering of the codomain curve of the arrow].

(ii) Let us write

$$
\mathcal{D}_{Y}^{\text {ell }} \subseteq \mathcal{D}_{Y} ; \quad \mathcal{D}_{W}^{\text {ell }} \subseteq \mathcal{D}_{W}
$$

for the full subcategories of tempered coverings that are unramified over the cusps of $Y^{\log }, W^{\log }$ [i.e., the "tempered coverings of the underlying elliptic curve of $\left.Y^{\text {log" }}\right]$. Thus, by taking the left adjoints to the natural inclusion functors $\mathcal{D}_{Y}^{\text {ell }} \hookrightarrow \mathcal{D}_{Y}, \mathcal{D}_{W}^{\text {ell }} \hookrightarrow \mathcal{D}_{W}$, we obtain natural functors $\mathcal{D}_{Y} \rightarrow \mathcal{D}_{Y}^{\text {ell }}, \mathcal{D}_{W} \rightarrow \mathcal{D}_{W}^{\text {ell }}$ [cf. [Mzk18], Example 1.3, (ii)], as well as 1-commutative diagrams of natural functors
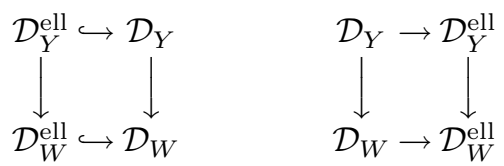
[since $Y^{\log } \rightarrow W^{\log }$ is unramified at the cusps of $Y^{\log }$ ].

(iii) Next, let us denote by $\Phi_{\mathcal{W}}$ the monoid on $\mathcal{D}_{W}$ given by forming the perfection of the monoid " $\Phi_{0}$ " of Definition 3.3, (iii), for some choice of tempered filter on $W^{\log }$ that arises from a tempered filter on $Y^{\log }$ [i.e., whose constituent subgroups $\subseteq \underline{\Delta}_{W}^{\mathrm{tp}}$ are contained in $\left.\underline{\Delta}_{Y}^{\mathrm{tp}} \subseteq \Delta_{W}^{\mathrm{tp}}\right]$. Now define

$$
\left.\Phi_{W^{\text {ell }}}^{\text {ell }} \subseteq \Phi_{\mathcal{W}}\right|_{\mathcal{D}_{W}^{\text {ell }}}
$$

as follows: For $A \in \operatorname{Ob}\left(\mathcal{D}_{W}^{\text {ell }}\right)$, we take $\Phi_{W}^{\text {ell }}(A)$ to be the perf-saturation [cf. $\S 0]$ in $\Phi_{\mathcal{W}}(A)$ of the submonoid

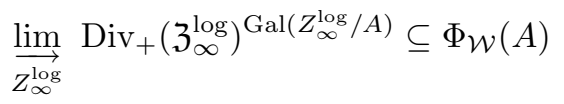

- where $Z_{\infty}^{\log }$ ranges over the connected tempered coverings $Z_{\infty}^{\log } \rightarrow A$ in $\mathcal{D}_{W}^{\text {ell }}$ such that the composite covering $Z_{\infty}^{\log } \rightarrow A \rightarrow W^{\log }$ arises as the generic fiber of the "universal combinatorial covering" $\mathfrak{Z}_{\infty}^{\log }$ of the stable logarithmic model $\mathfrak{Z}^{\log }$ of some finite $\log$ étale Galois covering $Z^{\log } \rightarrow W^{\log }\left[\right.$ in $\mathcal{D}_{W}^{\text {ell }}$ ! with stable, split reduction over the ring of integers of a finite extension $L$ of $K$; the superscript Galois group denotes the submonoid of elements fixed by the Galois group in question. Here, we pause to observe that the various monoids that occur in the above inductive limit are all contained in $\Phi_{\mathcal{W}}(A)$, and that the induced morphisms on perf-saturations between these monoids are bijective [cf. Remark 3.3.1]. Indeed, this bijectivity follows immediately from the wellknown structure of the special fibers of the "universal combinatorial coverings" that appear in the above inductive limit [i.e., "chains of copies of the projective line" — cf., e.g., the discussion preceding Proposition 1.1]. Set

$$
\left.\left.\Phi_{W}^{\text {ell }} \stackrel{\text { def }}{=} \Phi_{W}^{\text {ell }}\right|_{\mathcal{D}_{W}} \subseteq\left(\left.\Phi_{\mathcal{W}}\right|_{\mathcal{D}_{W}^{\text {ell }}}\right)\right|_{\mathcal{D}_{W}} \subseteq \Phi_{\mathcal{W}}
$$

- where " $\left.\right|_{\mathcal{D}_{W}}$ " is with respect to the functor $\mathcal{D}_{W} \rightarrow \mathcal{D}_{W}^{\text {ell }}$ defined in (ii). Now observe that $\Phi_{W}^{\mathrm{ell}}$ is a perfect [cf. the definition of $\Phi_{W}^{\text {ell }}$ as a perf-saturation inside the perfect monoid $\left.\Phi_{\mathcal{W}}\right|_{\mathcal{D}_{W}^{\text {ell }}}$ on $\mathcal{D}_{W}^{\text {ell }}$ ] and [manifestly - cf. Remark 3.6.1] group-saturated submonoid of the monoid $\Phi_{\mathcal{W}}$ on $\mathcal{D}_{W}$, which is, moreover, perffactorial, non-dilating [cf. Proposition 3.4, (i); the above observation concerning the bijectivity of induced morphisms on perf-saturations], and cuspidally pure [cf. the well-known structure of the special fibers of the "universal combinatorial coverings" that appear in the above inductive limit]. Also, $\Phi_{W}^{\mathrm{ell}}$ is [manifestly] independent, up to natural isomorphism, of the choice of tempered filter on $W$ used to define $\Phi_{\mathcal{W}}$. 
(iv) If $\alpha: A \rightarrow B$ is any morphism of $\mathcal{D}_{W}$, then set

$$
\mathcal{D}_{\alpha} \stackrel{\text { def }}{=}\left(\mathcal{D}_{W}\right)_{B}[\alpha] \quad\left(\subseteq\left(\mathcal{D}_{W}\right)_{B}\right)
$$

- where we regard $\alpha$ as an object of $\left(\mathcal{D}_{W}\right)_{B}-$ cf. the notational conventions of $\S 0 ;$ [Mzk17], $\S 0$. Thus, $\mathcal{D}_{\alpha}^{\top}$ is a quasi-temperoid [cf. [Mzk14], Definition A.1, (ii)]. Also, we observe that $\mathcal{D}_{W}, \mathcal{D}_{X}, \mathcal{D}_{Y}, \mathcal{D}_{U}$ are special cases of " $\mathcal{D}_{\alpha}$ " [obtained by taking " $\alpha$ " be the identity morphism of $W^{\log }, X^{\log }, Y^{\log }, U^{\log }$ ]. Note that we have a natural functor $\mathcal{D}_{\alpha} \rightarrow \mathcal{D}_{W}$. Now let us write

$$
\left.\Phi_{\alpha}^{\text {ell }} \stackrel{\text { def }}{=} \Phi_{W}^{\text {ell }}\right|_{\mathcal{D}_{\alpha}}\left(\left.\subseteq \Phi_{\mathcal{W}}\right|_{\mathcal{D}_{\alpha}}\right)
$$

and note that it follows immediately from the above discussion that this [sub-] monoid $\Phi_{\alpha}^{\text {ell }}$ — which is obtained simply by restricting the functor $\Phi_{W}^{\mathrm{ell}}$ via some functor - is perfect, group-saturated, perf-factorial, non-dilating, and cuspidally pure. Note, moreover, that the existence of the theta functions discussed in $\S 1$ [cf. especially the description of the zeroes and poles of these theta functions given in Proposition 1.4, (i)] implies that the monoid $\Phi_{\alpha}^{\text {ell }}$ is also rational [cf. Definition 3.6, (ii); [Mzk17], Definition 4.5, (ii)]. In particular, it follows that:

This monoid $\Phi_{\alpha}^{\mathrm{ell}}$ [along with its perfection and realification - cf. Remark 3.6.4] gives rise to a tempered Frobenioid [cf. Definition 3.6, (ii); Remark 3.6.6] of rationally standard type [cf. Theorem 3.7, (ii)] with perfect divisor monoid over the slim [cf. Remark 3.7.2] base category $\mathcal{D}_{\alpha}$ of FSM-type [cf. Remark 3.7.2].

\section{§4. General Bi-Kummer Theory}

In the present $\S 4$, we apply the theory of tempered Frobenioids developed in $\S 3$ to discuss the analogue, for log-meromorphic functions on tempered coverings of smooth log orbicurves over nonarchimedean [mixed-characteristic] local fields, of the Kummer theory for p-adic Frobenioids developed in [Mzk18], $\S 2$. One important aspect [i.e., in a word, the "bi" portion of the term "biKummer"] of the "bi-Kummer theory" theory developed here — by comparison, for instance, to the Kummer theory for arbitrary Frobenioids discussed in [Mzk18], Definition 2.1 - is that instead of just taking roots of the given log-meromorphic function, one considers roots of the pair of sections of a line bundle that correspond, respectively, to the "numerator" and "denominator" of the log-meromorphic function [cf. Remark 4.3 .1 below]. Another important feature of the theory developed here - by comparison to the theory of [Mzk18], 
$\S 2$, for $p$-adic Frobenioids - is the absence of an analogue of the reciprocity map [cf. Remark 4.4.1 below]. As we shall see in $\S 5$ below [cf. Theorem 5.6 and its proof], the additional "layer of complexity" that arises from the former feature has the effect of compensating [to a certain extent, at least in the case of the situation discussed in $\S 2]$ for the "handicap" constituted by the latter feature. Finally, we remark that the theory developed here may be regarded as and, indeed, was motivated by the goal of developing a Frobenioid-theoretic translation/generalization - via the theory of base-Frobenius pairs [cf. [Mzk17], Definition 2.7, (iii); [Mzk17], Proposition 5.6] — of the scheme-theoretic constructions of $\S 1$.

Let $X^{\log }, K, \mathcal{D}_{0}=\mathcal{B}^{\text {temp }}\left(X^{\log }\right)^{0}$ be as in $\S 3$. In the following discussion, we fix a tempered Frobenioid $\mathcal{C}$ whose monoid type is $\mathbb{Z}$, whose divisor monoid $\Phi$ is perfect, whose base category $\mathcal{D}$ is of the form

$$
\mathcal{D} \stackrel{\text { def }}{=} \mathcal{D}_{0}[D] \quad\left(\subseteq \mathcal{D}_{0}\right)
$$

[cf. $\S 0]$, where $D \in \operatorname{Ob}\left(\mathcal{D}_{0}\right)$, and whose base-field-theoretic hull we denote by $\mathcal{C}^{\text {bs-fld }} \subseteq \mathcal{C}$. Also, we fix a Frobenius-trivial object $A_{\odot} \in \mathrm{Ob}(\mathcal{C})$ such that $A_{\odot}^{\text {bs }} \stackrel{\text { def }}{=} \operatorname{Base}\left(A_{\odot}\right) \in \operatorname{Ob}(\mathcal{D})$ is a Galois [cf. [Mzk14], Definition 3.1, (iv)] object. Thus, $A_{\odot}^{\text {bs }}$ determines normal open subgroups

$$
H_{\odot} \subseteq \underline{\Pi}_{X}^{\mathrm{tp}} ; \quad H_{\odot}^{\mathrm{bs}-\mathrm{fld}} \subseteq G_{K}
$$

[i.e., $H_{\odot}^{\text {bs-fld }}$ is the image of $H_{\odot}$ in $G_{K}$ ] of the tempered fundamental group $\underline{\Pi}_{X}^{\text {tp }}$ of $X^{\log }$ and the quotient $\underline{\Pi}_{X}^{\mathrm{tp}} \rightarrow G_{K}$ determined by the absolute Galois group of $K$. In the following discussion, we shall use the superscript "birat" (respectively, "bs") to denote the object or arrow determined by a given object or arrow of $\mathcal{C}$ in the birationalization $\mathcal{C}^{\text {birat }}$ [cf. [Mzk17], Proposition 4.4] (respectively, base category $\mathcal{D}$ ) of $\mathcal{C}$.

Recall that pre-steps of $\mathcal{C}$ map to isomorphisms in $\mathcal{C}^{\text {birat }}$ [cf. [Mzk17], Proposition 4.4, (iv)]. In particular, it follows that any base-equivalent pair of pre-steps $s^{\sqcap}, s^{\sqcup}: A \rightarrow B$ in $\mathcal{C}$ determines, by inverting the image of $s^{\sqcup}$ in $\mathcal{C}^{\text {birat }}$, an element " $s \sqcap \cdot\left(s^{\sqcup}\right)^{-1}$ " $\in \mathcal{O}^{\times}\left(A^{\text {birat }}\right)$.

Definition 4.1. Let $A \in \mathrm{Ob}(\mathcal{C})$.

(i) If $f \in \mathcal{O}^{\times}\left(A^{\text {birat }}\right)$, then we shall refer to as a fraction-pair, or, alternatively, as a right fraction-pair [for $f]$, any base-equivalent pair of pre-steps

$$
s^{\sqcap}, s^{\sqcup}: A \rightarrow B
$$

such that $s^{\sqcap} \cdot\left(s^{\sqcup}\right)^{-1}=f \in \mathcal{O}^{\times}\left(A^{\text {birat }}\right)$, and, moreover, $\operatorname{Div}\left(s^{\square}\right), \operatorname{Div}\left(s^{\sqcup}\right)$ have disjoint supports [cf. [Mzk17], Proposition 4.1, (iii)]. [Thus, $\operatorname{Div}\left(s^{\sqcap}\right), \operatorname{Div}\left(s^{\sqcup}\right)$ 
are uniquely determined by $f$.] In this situation, we shall refer to $\operatorname{Div}\left(s^{\sqcap}\right)$ as the zero divisor and to $\operatorname{Div}\left(s^{\sqcup}\right)$ as the divisor of poles of the fraction-pair $\left(s^{\square}, s^{\sqcup}\right)$; we shall refer to $A$ (respectively, $B$ ) as the domain (respectively, codomain) of the fraction-pair $\left(s^{\sqcap}, s^{\sqcup}\right)$; if we denote by $\left.f\right|_{B} \in \mathcal{O}^{\times}\left(B^{\text {birat }}\right)$ the element determined by $\left(s^{\sqcap}, s^{\sqcup}\right)$ [cf. [Mzk17], Proposition 4.4, (iv)], then we shall refer to the pair $\left(s^{\sqcap}, s^{\sqcup}\right)$ as a left fraction-pair $\left[\right.$ for $\left.\left.f\right|_{B}\right]$.

(ii) We shall say that $A$ is Galois if $A^{\text {bs }} \in \operatorname{Ob}(\mathcal{D})$ is Galois. Suppose that $A$ is Galois. Then, by the definition of $\mathcal{D}$, there is a natural surjective outer homomorphism

$$
\underline{\Pi}_{X}^{\mathrm{tp}} \rightarrow \operatorname{Aut}_{\mathcal{D}}\left(A^{\mathrm{bs}}\right)
$$

[cf. the discussion of [Mzk18], Definition 2.2, (i), in the case of $p$-adic Frobenioids]; write

$$
H_{A^{\text {bs }}} \subseteq \operatorname{Aut}_{\mathcal{D}}\left(A^{\text {bs }}\right)
$$

for the image of $H_{\odot}$ via this surjection [which is well-defined, since $H_{\odot}$ is normal] and

$$
H_{A} \subseteq \operatorname{Aut}_{\mathcal{C}}(A) / \mathcal{O}^{\times}(A)
$$

for the inverse image of $H_{A^{\text {bs }}}$ via the natural injection $\operatorname{Aut}_{\mathcal{C}}(A) / \mathcal{O}^{\times}(A) \hookrightarrow$ $\operatorname{Aut}_{\mathcal{D}}\left(A^{\text {bs }}\right)$. If the natural injection $H_{A} \hookrightarrow H_{A^{\text {bs }}}$ is a bijection, then we shall say that $A$ is $H_{\odot}$-ample.

(iii) Suppose that $A$ is $H_{\odot}$-ample [hence, in particular, Galois], and that $f \in \mathcal{O}^{\times}\left(A^{\text {birat }}\right)$ is an element fixed by the natural action of $H_{A}$; let $N \in \mathbb{N}_{\geq 1}$. Then we shall say that $A$ is $\left(N, H_{\odot}, f\right)$-saturated if the following conditions are satisfied: (a) there exist pre-steps $A^{\prime \prime} \rightarrow A, A^{\prime \prime} \rightarrow A^{\prime}$ in $\mathcal{C}$, where $A^{\prime}$ is Frobenius-trivial [hence determines an object of the p-adic Frobenioid $\mathcal{C}^{\text {bs-fld }}$ cf. Remark 3.6.3], such that $A^{\prime}$ is $\left(N, H_{\odot}^{\text {bs-fld }}\right)$-saturated [cf. [Mzk18], Definition 2.2 , (ii)] as an object of $\mathcal{C}^{\text {bs-fld }}$; (b) there exists a $g \in \mathcal{O}^{\times}\left(A^{\text {birat }}\right)$ such that $g^{N}=f$.

(iv) We shall say that a morphism $\alpha: A \rightarrow B$ of $\mathcal{C}$ is of base-Frobenius type if there exist a subgroup $G \subseteq \operatorname{Aut}_{\mathcal{C}_{B}}(\alpha) \subseteq \operatorname{Aut}_{\mathcal{C}}(A)$ and a factorization $\alpha=\alpha^{\prime \prime} \circ \alpha^{\prime}$ such that the following conditions are satisfied: (a) $A$ is Frobeniustrivial, Galois, and $\boldsymbol{\mu}_{N}$-saturated [cf. [Mzk18], Definition 2.1, (i)], where $N \stackrel{\text { def }}{=}$ $\operatorname{deg}_{\mathrm{Fr}}(\alpha)$; (b) $G$ maps isomorphically to $\operatorname{Gal}\left(A^{\mathrm{bs}} / B^{\mathrm{bs}}\right) \subseteq \operatorname{Aut}_{\mathcal{D}}\left(A^{\mathrm{bs}}\right) ;(\mathrm{c}) \alpha^{\prime}$ is a base-identity endomorphism of Frobenius type; (d) $\alpha^{\prime \prime}$ is a pull-back morphism; (e) $G, \alpha^{\prime}, \alpha^{\prime \prime}$ arise from a base-Frobenius pair of $\mathcal{C}$ [cf. Theorem 3.7, (i); [Mzk17], Proposition 5.6]. In this situation, we shall refer to the subgroup $G$ and the factorization $\alpha=\alpha^{\prime \prime} \circ \alpha^{\prime}$ as being of base-Frobenius type. 
Remark 4.1.1. Note that in the situation of Definition 4.1, (iv), if $\alpha: A \rightarrow$ $B$ is of base-Frobenius type, then by applying Proposition 3.4, (ii), together with the factorization of [Mzk17], Definition 1.3, (iv), (a), one verifies easily that $A \rightarrow B$ is a categorical quotient [cf. [Mzk17], §0] of $A$ by the subgroup $G \cdot \boldsymbol{\mu}_{N}(A) \subseteq \operatorname{Aut}_{\mathcal{C}}(A)$ in the full subcategory of $\mathcal{C}$ determined by the Frobeniustrivial objects [cf. [Mzk17], Theorem 5.1, (iii)].

Proposition 4.2 (Construction of Bi-Kummer Data I: Roots of FractionPairs). $\quad$ In the notation of the above discussion, let $f \in \mathcal{O}^{\times}\left(A_{\odot}^{\text {birat }}\right) ; s^{\sqcap}, s^{\sqcup}$ : $A_{\odot} \rightarrow B_{\odot} a$ right fraction-pair for $f$ [i.e., a left fraction-pair for $\left.\left.f\right|_{B_{\odot}}\right]$; $N \in \mathbb{N}_{\geq 1}$. Then:

(i) A pair of morphisms $t^{\sqcap}, t^{\sqcup}: A_{\odot} \rightarrow C_{\odot}$ is a right fraction-pair for $f$ if and only if there exists a [necessarily unique] isomorphism $v: B_{\odot} \stackrel{\sim}{\rightarrow} C_{\odot}$ such that $t^{\square}=v \circ s^{\square}, t^{\sqcup}=v \circ s^{\sqcup}$.

(ii) A pair of morphisms $t^{\sqcap}, t^{\sqcup}: C_{\odot} \rightarrow B_{\odot}$ is a left fraction-pair for $\left.f\right|_{B_{\odot}}$ if and only if there exists a [necessarily unique] isomorphism v $: C_{\odot} \stackrel{\sim}{\rightarrow} A_{\odot}$ such that $t^{\square}=s^{\sqcap} \circ v, t^{\sqcup}=s^{\sqcup} \circ v$.

(iii) There exist commutative diagrams in $\mathcal{C}$
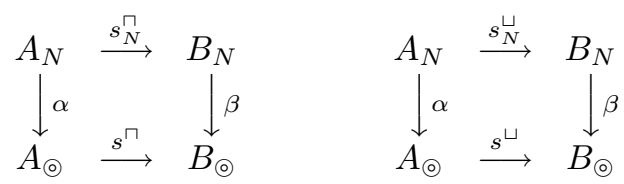

- where $\alpha, \beta$ are isometries of Frobenius degree $N ; \alpha$ is of baseFrobenius type, with factorization of base-Frobenius type $\alpha=\alpha^{\prime \prime} \circ \alpha^{\prime} ;\left.f\right|_{A_{N}} \stackrel{\text { def }}{=}$ $\left(\left(\alpha^{\prime \prime}\right)^{\text {birat }}\right)^{*}(f)\left[c f . \quad[\right.$ Mzk17], Proposition 1.11, $(i v)] ; A_{N}$ is $\left(N, H_{\odot},\left.f\right|_{A_{N}}\right)$ saturated; $s_{N}^{\sqcap}, s_{N}^{\sqcup}: A_{N} \rightarrow B_{N}$ are base-equivalent pre-steps. In particular, there exists an element $\in \mathcal{O}^{\times}\left(A_{N}^{\text {birat }}\right)$ (respectively, $\in \mathcal{O}^{\times}\left(B_{N}^{\text {birat }}\right)$ ) for which $\left(s_{N}^{\Gamma}, s_{N}^{\sqcup}\right)$ is a right (respectively, left) fraction-pair, and whose $N$-th power is equal to $\left.f\right|_{A_{N}}$ (respectively, $\left.\left.f\right|_{B_{N}} \stackrel{\text { def }}{=}\left(\left.f\right|_{A_{N}}\right)\right|_{B_{N}}[c f$. the notation of Definition $4.1,(i)])$. In the following, we shall refer to such a pair of commutative diagrams as an $N$-th root of the fraction-pair $\left(s^{\sqcap}, s^{\sqcup}\right)$, to $A_{N}$ as the $N$-domain of this root of a fraction-pair, and to $B_{N}$ as the $N$-codomain of this root of a fraction-pair.

(iv) We continue to use the notation of (iii). Let

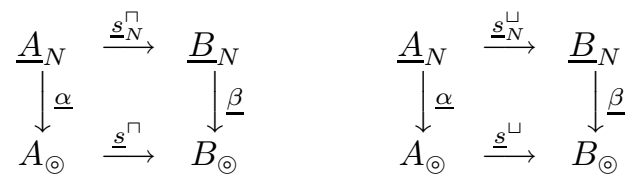


be another $N$-th root of a left fraction-pair $\left(\underline{s}^{\square}, \underline{s}^{\sqcup}\right)$ for $\left.f\right|_{B_{\odot}} ; \delta \in \operatorname{Aut}_{\mathcal{C}}\left(A_{\odot}\right)$ the unique automorphism $\left[\right.$ cf. (ii)] such that $\underline{s} \square=s^{\square} \circ \delta, \underline{s}^{\sqcup}=s^{\sqcup} \circ \delta ; \epsilon_{A}$ : $A_{N}^{\mathrm{bs}} \stackrel{\sim}{\rightarrow} \underline{A}_{N}^{\mathrm{bs}}$ an isomorphism of $\mathcal{D}$ such that $\alpha^{\mathrm{bs}}=\delta^{\mathrm{bs}} \circ \underline{\alpha}^{\mathrm{bs}} \circ \epsilon_{A}$. Then, after possibly replacing $s_{N}^{\sqcap}$ by $u \circ s_{N}^{\sqcap}$, for some $u \in \boldsymbol{\mu}_{N}\left(B_{N}\right)\left[\right.$ where " $\boldsymbol{\mu}_{N}(-)$ " is as in [Mzk18], Definition 2.1, (i)], there exist isomorphisms $\zeta_{A}: A_{N} \stackrel{\sim}{\rightarrow} \underline{A}_{N}$, $\zeta_{B}: B_{N} \stackrel{\sim}{\rightarrow} \underline{B}_{N}$ in $\mathcal{C}$ which fit into commutative diagrams
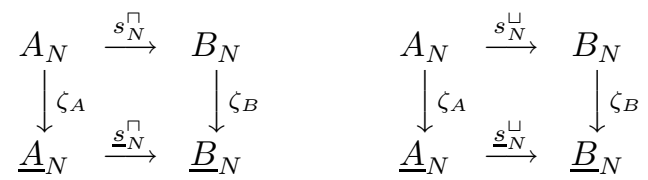

and, moreover, satisfy $\alpha=\delta \circ \underline{\alpha} \circ \zeta_{A}, \beta=\underline{\beta} \circ \zeta_{B}, \zeta_{A}^{\mathrm{bs}}=\epsilon_{A}$. Here, $\zeta_{B}$ is uniquely determined by $\zeta_{A} ; \zeta_{A}$ is uniquely determined by $\epsilon_{A}$, up to composition with an element of $\boldsymbol{\mu}_{N}\left(A_{N}\right)$. [Thus, $\zeta_{B}$ is uniquely determined by $\epsilon_{A}$, up to composition with an element of $\boldsymbol{\mu}_{N}\left(B_{N}\right)$.] In the following, we shall refer to such a pair $\left(\zeta_{A}, \zeta_{B}\right)$ as an isomorphism between the two given $N$-th roots of fraction-pairs.

Proof. The sufficiency portion of assertion (i) is immediate. To verify the necessity portion of assertion (i), observe that by the equivalences of categories of [Mzk17], Definition 1.3, (iii), (d), the "disjoint supports" condition [cf. Definition 4.1, (i)] on the Div(-)'s of the components of a fraction-pair $t^{\sqcap}, t^{\sqcup}: A_{\odot} \rightarrow C_{\odot}$ implies the existence of isomorphisms $v, v^{\prime}: B_{\odot} \stackrel{\sim}{\rightarrow} C_{\odot}$ such that $t^{\sqcap}=v \circ s^{\sqcap}, t^{\sqcup}=v^{\prime} \circ s^{\sqcup}$; since, moreover, $t^{\sqcap}, t^{\sqcup}$ are base-equivalent, it follows that $v=v^{\prime \prime} \circ v^{\prime}$, for some $v^{\prime \prime} \in \mathcal{O}^{\times}\left(C_{\odot}\right)$. On the other hand, since the fraction-pair $t^{\sqcap}, t^{\sqcup}: A_{\odot} \rightarrow C_{\odot}$ determines the same element of $\mathcal{O}^{\times}\left(A_{\odot}^{\text {birat }}\right)$ as the fraction-pair $s^{\sqcap}, s^{\sqcup}: A_{\odot} \rightarrow C_{\odot}$, we thus conclude that $v^{\prime \prime}=1$. Note that the uniqueness of $v$ follows from the total epimorphicity of $\mathcal{C}$. This completes the proof of assertion (i). The proof of assertion (ii) is entirely similar [except that one concludes uniqueness from the fact that pre-steps are always monomorphisms - cf. [Mzk17], Definition 1.3, (v), (a)].

Next, we consider assertions (iii), (iv). First, let us observe that, by the definition of "log-meromorphic" [cf. Definition 3.1, (ii)], it follows immediately that over some tempered covering of $X^{\log }$ that occurs as the "universal combinatorial covering" of a finite étale covering of $X^{\log }$ with stable, split reduction, $f$ admits an $N$-th root. Since, moreover, the divisor monoid $\Phi$ is assumed to be perfect, it follows that the divisors of zeroes and poles of such an $N$-th root belong to $\Phi(-)$ of the tempered covering in question. Now since the Frobenioid $\mathcal{C}$ is of model [hence, in particular, pre-model — cf. [Mzk17], Definition 4.5, (i)] 
type [cf. Theorem 3.7, (i)], it follows that $\mathcal{C}$ admits a base-Frobenius pair [cf. [Mzk17], Definition 2.7, (iii)]. Thus, the existence of a pair of commutative diagrams as in the statement of assertion (iii) follows by translating the above "scheme-theoretic observations" into the language of Frobenioids — cf. [Mzk17], Definition 1.3, (iii), (d) [on the existence of pre-steps with prescribed zero divisor]; [Mzk18], Remark 2.2.1 [concerning the issue of “( $\left.N, H_{\odot}^{\mathrm{bs}-f l d}\right)$-saturation"].

Finally, to verify the uniqueness up to isomorphism of such a pair of commutative diagrams as stated in assertion (iv), let us first observe that by replacing $\alpha, s^{\sqcap}, s^{\sqcup}$ by $\delta^{-1} \circ \alpha, s^{\sqcap} \circ \delta, s^{\sqcup} \circ \delta$, respectively, we may assume without loss of generality that $\delta$ is the identity. Next, let us observe that it follows from the " $\left(N, H_{\odot},\left.f\right|_{A_{N}}\right)$-saturated-ness" condition in the statement of assertion (iii) [cf. also Proposition 3.4, (ii)] that the pull-back $\in \mathcal{O}^{\times}\left(A_{N}\right)$ or $\in \mathcal{O}^{\times}\left(\underline{A}_{N}\right)$ of any element $\in \mathcal{O}^{\times}\left(A_{\odot}\right)$ [i.e., via the "pull-back portion" of $\alpha, \underline{\alpha}-$ cf. Definition 4.1, (iv), (d)] admits an $N$-th root. Now [in light of this observation] the existence of a $\zeta_{A}$ as desired follows immediately from the uniqueness up to conjugation by a unit of base-Frobenius pairs of $A_{N}, \underline{A}_{N}$ [cf. [Mzk17], Proposition 5.6], by thinking of $\alpha, \underline{\alpha}$ as categorical quotients [cf. Remark 4.1.1] and applying the base-triviality and Aut-ampleness of the full subcategory of $\mathcal{C}$ determined by the Frobenius-trivial objects [cf. [Mzk17], Theorem 5.1, (iii)]. The existence of a $\zeta_{B}$ as desired follows from the equivalences of categories determined by pre-steps of [Mzk17], Definition 1.3, (iii), (d). The essential uniqueness of $\zeta_{A}$, $\zeta_{B}$ as asserted follows immediately from the various conditions imposed on $\zeta_{A}$, $\zeta_{B}$. This completes the proof of assertion (iv).

Proposition 4.3 (Construction of Bi-Kummer Data II: Bi-Kummer Roots). In the notation of Proposition 4.2, (iii):

(i) Let

$$
s_{N}^{\operatorname{trv}}: H_{A_{N}} \rightarrow \operatorname{Aut}_{\mathcal{C}}\left(A_{N}\right)
$$

be the group homomorphism arising from a base-Frobenius pair of $A_{N}[c f$. Theorem 3.7, (i); [Mzk17], Proposition 5.6]. [Thus, $s_{N}^{\mathrm{trv}}$ is completely determined up to conjugation by an element of $\mathcal{O}^{\times}\left(A_{N}\right)-c f$. Theorem 3.7, $(i)$; [Mzk17], Proposition 5.6.] Then there exist unique group homomorphisms

$$
s_{N}^{\sqcap \text {-gp }}: H_{B_{N}} \rightarrow \operatorname{Aut}_{\mathcal{C}}\left(B_{N}\right) ; \quad s_{N}^{\sqcup-\mathrm{gp}}: H_{B_{N}} \rightarrow \operatorname{Aut}_{\mathcal{C}}\left(B_{N}\right)
$$

such that, relative to the isomorphism $H_{A_{N}} \stackrel{\sim}{\rightarrow} H_{B_{N}}$ determined by the [baseequivalent!] pair of morphisms $s_{N}^{\square}, s_{N}^{\sqcup}$, we have

$$
s_{N}^{\sqcap-\mathrm{gp}}(h) \circ s_{N}^{\sqcap}=s_{N}^{\sqcap} \circ\left(\left.s_{N}^{\mathrm{trv}}\right|_{H_{B_{N}}}\right)(h) ; \quad s_{N}^{\sqcup-\mathrm{gp}}(h) \circ s_{N}^{\sqcup}=s_{N}^{\sqcup} \circ\left(\left.s_{N}^{\mathrm{trv}}\right|_{H_{B_{N}}}\right)(h)
$$


for all $h \in H_{B_{N}}$. [In particular, it follows that $B_{N}$ is $H_{\odot}$-ample. $]$ In the following, we shall refer to such a pair $\left(s_{N}^{\sqcap-\mathrm{gp}}, s_{N}^{\sqcup-\mathrm{gp}}\right)$ as a bi-Kummer $N$-th root of the fraction-pair $\left(s^{\sqcap}, s^{\sqcup}\right)$; also, we shall speak of $A_{N}, B_{N}$ as being the "N-domain", "N-codomain", respectively, [cf. Proposition 4.2, (iii)] not only of the original root of a fraction-pair, also of the resulting bi-Kummer root.

(ii) In the notation of $(i)$, the collection of bi-Kummer $N$-th roots, with $N$-codomain $B_{N}$, of a fraction-pair with domain isomorphic to $A_{\odot}$, of some rational function whose pull-back to [the $N$-codomain] $B_{N}$ is equal to $\left.f\right|_{B_{N}}$, is equal to the collection of pairs obtained from $\left(s_{N}^{\sqcap-\mathrm{gp}}, s_{N}^{\sqcup-\mathrm{gp}}\right)$ by

(a) simultaneous conjugation by an element $\zeta_{\text {Aut }} \in \mathcal{O}^{\times}\left(B_{N}\right)$, followed by

(b) non-simultaneous conjugation [of, say, $s_{N}^{\sqcap-\mathrm{gp}}$, but not $\left.s_{N}^{\sqcup-\mathrm{gp}}\right]$ by an element $u \in \boldsymbol{\mu}_{N}\left(B_{N}\right)$ [cf. the "u" of Proposition 4.2, (iv)].

(iii) In the notation of $(i)$, the difference $s_{N}^{\sqcap-\mathrm{gp}} \cdot\left(s_{N}^{\sqcup-\mathrm{gp}}\right)^{-1}$ determines a twisted homomorphism $H_{B_{N}} \rightarrow \boldsymbol{\mu}_{N}\left(B_{N}\right)$, hence an element of the cohomology module $H^{1}\left(H_{B_{N}}, \boldsymbol{\mu}_{N}\left(B_{N}\right)\right)$, which is equal to the Kummer class [cf. [Mzk18], Definition 2.1, (ii)]

$$
\kappa_{\left.f\right|_{B_{N}}} \in H^{1}\left(H_{B_{N}}, \boldsymbol{\mu}_{N}\left(B_{N}\right)\right)
$$

of $\left.f\right|_{B_{N}}$ [cf. the notation of Proposition 4.2, (iii)]. In particular, this cohomology class is independent of the [simultaneous and non-simultaneous] conjugation operations discussed in (ii).

Proof. First, we consider assertion (i). To prove the existence and uniqueness of $s_{N}^{\sqcap \text {-gp }}, s_{N}^{\sqcup-\mathrm{gp}}$, it follows from the general theory of Frobenioids - cf. the first equivalence of categories involving pre-steps of [Mzk17], Definition 1.3, (iii), (d); the fact that Frobenioids are always totally epimorphic - that it suffices to prove that

$$
\operatorname{Div}\left(s_{N}^{\sqcap}\right), \operatorname{Div}\left(s_{N}^{\sqcup}\right) \in \Phi\left(A_{N}\right)
$$

are fixed by $H_{A_{N}}$. But since $N \cdot \operatorname{Div}\left(s_{N}^{\sqcap}\right), N \cdot \operatorname{Div}\left(s_{N}^{\sqcup}\right) \in \Phi\left(A_{N}\right)$ arise as pullbacks to $A_{N}$ of elements of $\Phi\left(A_{\odot}\right)$ [i.e. $\operatorname{Div}\left(s^{\sqcap}\right), \operatorname{Div}\left(s^{\sqcup}\right)$ ], this follows from the fact that [by definition] $H_{\odot}$ acts trivially on $A_{\odot}^{\text {bs }}$ [together with the fact the monoid $\Phi\left(A_{N}\right)$ is torsion-free!]. This completes the proof of assertion (i). Assertion (iii) follows immediately from the definitions [cf., especially, [Mzk18], Definition 2.1, (ii)].

Finally, we observe that assertion (ii) follows immediately by applying Proposition 4.2, (ii), to the fraction-pairs $\left(s_{N}^{\square}, s_{N}^{\sqcup}\right)$ and $\left(\underline{s}_{N}^{\square}, \underline{s}_{N}^{\sqcup}\right)$ of Proposition 
4.2, (iv) [after possibly replacing $s_{N}^{\square}$ by $u \circ s_{N}^{\square}$ ]. Indeed, since $s_{N}^{\text {trv }}$ is completely determined up to conjugation by an element of $\mathcal{O}^{\times}\left(A_{N}\right)$ [cf. Theorem 3.7, (i); [Mzk17], Proposition 5.6.], we thus conclude that the collection of bi-Kummer $N$-th roots under consideration is as stated in assertion (ii). This completes the proof of assertion (ii).

Remark 4.3.1. Of course, even without applying the somewhat nontrivial theory of base-Frobenius pairs [i.e., [Mzk17], Proposition 5.6], liftings to $\operatorname{Aut}_{\mathcal{C}}\left(A_{N}\right)$ of individual elements of $H_{A_{N}}$ [cf. the notation of Proposition 4.3 , (i)] are completely determined up to possible translation by elements of $\mathcal{O}^{\times}\left(A_{N}\right)$. The crucial difference, however, between an indeterminacy up to translation by elements of $\mathcal{O}^{\times}\left(A_{N}\right)$ and an indeterminacy up to conjugation by elements of $\mathcal{O}^{\times}\left(A_{N}\right)$ is that, unlike the former, the latter allows one to work with sections [i.e., $s_{N}^{\text {trv }}, s_{N}^{\sqcap \text {-gp }}, s_{N}^{\sqcup \text {-gp }}$ ] which are group homomorphisms. Of course, the Kummer class [which, by Proposition 4.3, (iii), is determined by the pair $\left.\left(s_{N}^{\Pi-\mathrm{gp}}, s_{N}^{\sqcup-\mathrm{gp}}\right)\right]$ is always a [twisted] homomorphism. On the other hand, the theory of base-Frobenius pairs allows one to work with group homomorphisms [i.e., $s_{N}^{\sqcap \text {-gp }}$ or $s_{N}^{\sqcup \text {-gp }}$ ] even when one is forced to restrict one's attention to only one of the two arrows $s_{N}^{\sqcap}$ or $s_{N}^{\sqcup}$, i.e., in situations where one is not allowed to work with the fraction-pair as a "single entity" — cf. the theory of "Frobenioid-theoretic mono-theta environments" developed in Theorem 5.10 below.

Remark 4.3.2. Note that if $N$ divides $N^{\prime} \in \mathbb{N}_{\geq 1}$, then one verifies immediately that given an $N$-th root of the fraction-pair $\left(s^{\sqcap}, s^{\sqcup}\right)$ [e.g., as in Proposition 4.2 , (iii)], there exists a "morphism" from a suitable $N^{\prime}$-th root of the fractionpair $\left(s^{\sqcap}, s^{\sqcup}\right)$

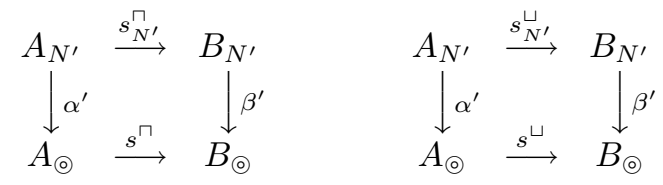

to the given $N$-th root of the fraction-pair $\left(s^{\sqcap}, s^{\sqcup}\right)$, i.e., a pair of commutative diagrams

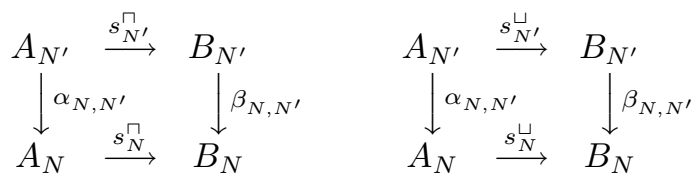


- where $\alpha_{N, N^{\prime}}$ (respectively, $\beta_{N, N^{\prime}}$ ) is an isometry of Frobenius degree $N^{\prime} / N$ that is compatible with $\alpha^{\prime}, \alpha$ (respectively, $\left.\beta^{\prime}, \beta\right) ; \alpha_{N, N^{\prime}}$ is of base-Frobenius type. For instance, such a "morphism" may be constructed by extracting an " $N^{\prime} / N$-th root" [cf. Proposition 4.2, (iii)] of the [fraction-pair determined by the] given " $N$-th root" of the fraction-pair $\left(s^{\sqcap}, s^{\sqcup}\right)$. Moreover, this collection of data induces a natural morphism

$$
H^{1}\left(H_{B_{N}}, \boldsymbol{\mu}_{N}\left(B_{N}\right)\right) \rightarrow H^{1}\left(H_{B_{N^{\prime}}}, \boldsymbol{\mu}_{N}\left(B_{N^{\prime}}\right)\right)
$$

that maps $\kappa_{\left.f\right|_{B_{N}}}$ to the image of $\kappa_{\left.f\right|_{B_{N^{\prime}}}}$ in $H^{1}\left(H_{B_{N^{\prime}}}, \boldsymbol{\mu}_{N}\left(B_{N^{\prime}}\right)\right)$ [i.e., via the natural surjection $\left.\boldsymbol{\mu}_{N^{\prime}}\left(B_{N^{\prime}}\right) \rightarrow \boldsymbol{\mu}_{N}\left(B_{N^{\prime}}\right)\right]$. In particular, by allowing $N$ to vary, we obtain

$$
\text { a compatible system of roots of the fraction-pair }\left(s^{\square}, s^{\sqcup}\right)
$$

hence a compatible system of Kummer classes, which, by Proposition 3.2, (iii), is sufficient to distinguish $f$ from other elements of $\mathcal{O}^{\times}\left(A_{\odot}^{\text {birat }}\right)$.

We conclude our discussion of "general bi-Kummer theory" by observing that, up to the various indeterminacies discussed so far, our constructions are entirely "category-theoretic":

Theorem 4.4 (Category-theoreticity of Bi-Kummer Data). For $i=$ 1,2 , let $p_{i}$ be a prime number; $K_{i}$ a finite extension of $\mathbb{Q}_{p_{i}} ; X_{i}^{\log }$ a smooth log orbicurve over $K_{i} ; \mathcal{C}_{i}$ a tempered Frobenioid, whose monoid type is $\mathbb{Z}$, whose divisor monoid $\Phi_{i}$ is perfect and non-dilating, and whose base category $\mathcal{D}_{i}$ is of the form

$$
\mathcal{D}_{i} \stackrel{\text { def }}{=} \mathcal{B}^{\text {temp }}\left(X_{i}^{\log }\right)\left[D_{i}\right]
$$

where $D_{i} \in \operatorname{Ob}\left(\mathcal{B}^{\text {temp }}\left(X_{i}^{\log }\right)\right) ; A_{\odot, i} \in \operatorname{Ob}\left(\mathcal{C}_{i}\right)$ a Frobenius-trivial object such that $A_{\odot, i}^{\text {bs }} \stackrel{\text { def }}{=} \operatorname{Base}\left(A_{\odot, i}\right) \in \operatorname{Ob}\left(\mathcal{D}_{i}\right)$ is Galois, hence determines a normal open subgroup

$$
H_{\odot, i} \subseteq \underline{\Pi}_{X_{i}}^{\mathrm{tp}}
$$

of the tempered fundamental group $\underline{\Pi}_{X_{i}}^{\mathrm{tp}}$ of $X_{i}^{\log } ; N \in \mathbb{N}_{\geq 1}$. Suppose that

$$
\Psi: \mathcal{C}_{1} \stackrel{\sim}{\rightarrow} \mathcal{C}_{2}
$$

is an equivalence of categories which induces [cf. Theorem 3.7, (i), (ii); Remark 3.7.2; [Mzk17], Theorem 3.4, (v)] an equivalence $\Psi^{\mathrm{bs}}: \mathcal{D}_{1} \stackrel{\sim}{\rightarrow} \mathcal{D}_{2}$ that maps $A_{\odot, 1}^{\mathrm{bs}}$ to an isomorph $[c f . \S 0]$ of $A_{\odot, 2}^{\mathrm{bs}}$. Then: 
(i) $\Psi$ maps isomorphs of $A_{\odot, 1}$ to isomorphs of $A_{\odot, 2}$ and $H_{\odot, 1}$-ample objects to $H_{\odot, 2}$-ample objects; $\Psi^{\text {bs }}$ induces an isomorphism $H_{\odot, 1} \stackrel{\sim}{\rightarrow} H_{\odot, 2}$, which is well-defined up to composition with inner automorphisms of $\underline{\Pi}_{X_{i}}^{\mathrm{tp}}$.

(ii) $\Psi$ induces a 1-compatible equivalence of categories $\Psi^{\text {birat }}: \mathcal{C}_{1}^{\text {birat }} \stackrel{\sim}{\rightarrow}$ $\mathcal{C}_{2}^{\text {birat }}$. Moreover, $\Psi$ preserves fraction-pairs, right fraction-pairs, and left fraction-pairs. Finally, $\Psi$ maps $N$-th roots of fraction-pairs with domain isomorphic to $A_{\odot, 1}$ to $N$-th roots of fraction-pairs with domain isomorphic to $A_{\odot, 2}$.

(iii) For $i=1,2$, let $A_{i} \in \mathrm{Ob}\left(\mathcal{C}_{i}\right)$ be $H_{\odot, i}$-ample; $f_{i} \in \mathcal{O}^{\times}\left(A_{i}^{\text {birat }}\right)$ an element fixed by the natural action of $H_{A_{i}}\left[\right.$ where " $H_{A_{i}}$ " is defined as in Definition 4.1, (ii), by taking " $H_{\odot}$ " to be $\left.H_{\odot, i}\right] ; N \in \mathbb{N}_{\geq 1}$. Suppose that $A_{1} \mapsto A_{2}$ via $\Psi$, and that $f_{1} \mapsto f_{2}$ via $\Psi^{\text {birat }}$. Then $A_{1}$ is $\left(N, H_{\odot, 1}, f_{1}\right)$-saturated if and only if $A_{2}$ is $\left(N, H_{\odot, 2}, f_{2}\right)$-saturated. Suppose that, for $i=1,2, A_{i}$ is $\left(N, H_{\odot, i}, f_{i}\right)$-saturated. Then the isomorphism

$$
H^{1}\left(H_{A_{1}}, \boldsymbol{\mu}_{N}\left(A_{1}\right)\right) \stackrel{\sim}{\rightarrow} H^{1}\left(H_{A_{2}}, \boldsymbol{\mu}_{N}\left(A_{2}\right)\right)
$$

maps $\kappa_{f_{1}} \mapsto \kappa_{f_{2}}$, i.e., is compatible with the Kummer classes of [Mzk18], Definition 2.1, (ii).

(iv) For $i=1,2$, let $B_{i} \in \mathrm{Ob}\left(\mathcal{C}_{i}\right)$ be an $N$-codomain of an $N$-th root of a fraction-pair with domain isomorphic to $A_{\odot, i}$; write

$$
s_{i}^{\sqcap \text {-gp }}, s_{i}^{\sqcup-\mathrm{gp}}: H_{B_{i}} \rightarrow \operatorname{Aut}_{\mathcal{C}_{i}}\left(B_{i}\right)
$$

for the corresponding bi-Kummer $N$-th root $[c f$. Proposition 4.3, $(i)]$ and $f_{i} \in \mathcal{O}^{\times}\left(B_{i}^{\text {birat }}\right)$ for the restriction to $B_{i}\left[\right.$ i.e., " $\left.f\right|_{B_{N}}$ " in the notation of Proposition 4.2, (iii)] of the rational function determined by the original fractionpair. Suppose that $B_{1} \mapsto B_{2}$ via $\Psi$, and that $f_{1} \mapsto f_{2}$ via $\Psi^{\text {birat }}$. Then, up to the [simultaneous and non-simultaneous] conjugation operations discussed in Proposition 4.3, (ii), the isomorphisms

$$
H_{B_{1}} \stackrel{\sim}{\rightarrow} H_{B_{2}} ; \quad \operatorname{Aut}_{\mathcal{C}_{1}}\left(B_{1}\right) \stackrel{\sim}{\rightarrow} \operatorname{Aut}_{\mathcal{C}_{2}}\left(B_{2}\right)
$$

map $s_{1}^{\sqcap \text {-gp }} \mapsto s_{2}^{\sqcap \text {-gp }}, s_{1}^{\sqcup \text {-gp }} \mapsto s_{2}^{\sqcup \text {-gp }}$, i.e., $\Psi$ is compatible with bi-Kummer $N$-th roots.

Proof. First, we observe that, for $i=1,2, \mathcal{C}_{i}$ is of standard and isotropic type, but not of group-like type [cf. Theorem 3.7, (i), (ii)]; the base category $\mathcal{D}_{i}$ of $\mathcal{C}_{i}$ is slim [cf. Remark 3.7.2]. Thus, $\Psi$ preserves pre-steps, morphisms of Frobenius type, Frobenius degrees, isometries, and base-Frobenius pairs [cf. 
[Mzk17], Theorem 3.4, (ii), (iii); [Mzk17], Corollary 5.7, (i), (iv)]. In particular, [cf. also the existence of $\left.\Psi^{\text {bs }}\right] \Psi$ preserves Frobenius-trivial objects. Since the Frobenioid determined by the Frobenius-trivial objects of $\mathcal{C}_{i}$ is of base-trivial type [cf. [Mzk17], Theorem 5.1, (iii)], these observations [together with the existence of $\Psi^{\text {bs }}$ imply the portion of assertion (i) concerning $\Psi$. The portion of assertion (i) concerning $\Psi^{\text {bs }}$ follows immediately from the theory of [quasi-] temperoids [cf. [Mzk14], Proposition 3.2; [Mzk14], Theorem A.4].

Next, we consider assertions (ii), (iii). The existence of $\Psi^{\text {birat }}$ follows from [Mzk17], Corollary 4.10. Next, let us recall that $\Psi$ is compatible with the operation of passing to the perfection [cf. Theorem 3.7, (i), (ii); [Mzk17], Theorem 3.4, (iii)]. In particular, it follows immediately from the category-theoreticity of sets of primes given in [Mzk17], Theorem 4.2, (ii), that $\Psi$ preserves baseequivalent [cf. the existence of $\Psi^{\text {bs }}$ !] pairs of pre-steps whose Div(-)'s have disjoint supports. The remainder of assertions (ii), (iii) then follows immediately from the observations thus far, the existence of $\Psi^{\text {bs }}$, and the "manifestly category-theoretic nature" of " $\left(N, H_{\odot, i}^{\mathrm{bs}-\mathrm{fld}}\right)$-saturation" [cf. [Mzk18], Definition 2.2, (ii); [Mzk2], Lemma 1.3.8] and Kummer classes [cf. [Mzk18], Definition 2.1, (ii)]. This completes the proof of assertions (ii), (iii).

Finally, we consider assertion (iv). Observe that $\Psi$ preserves base-Frobenius pairs of Frobenius-trivial objects and [by assertion (ii)] maps $N$-th roots of fraction-pairs with domain isomorphic to $A_{\odot, 1}$ to $N$-th roots of fraction-pairs with domain isomorphic to $A_{\odot, 2}$; $\Psi^{\text {birat }}$ maps $f_{1} \mapsto f_{2}$. Thus, assertion (iv) follows formally from Proposition 4.3, (i), (ii).

Remark 4.4.1. Observe that one crucial difference between the bi-Kummer theory for tempered Frobenioids considered here and the theory of [Mzk18], $\S 2$, in the case of $p$-adic Frobenioids is that in the present case, there is no reciprocity map. This fact may be regarded as a reflection of the fact that the tempered group $\underline{\Pi}_{X}^{\mathrm{tp}}$ is not a profinite group of cohomological dimension 2 whose cohomology admits a duality theory of the sort that $G_{K}$ does. Put another way, although at first glance, the Kummer classes of Proposition 4.3, (iii); Theorem 4.4, (iii), may appear to constitute a "purely [tempered] fundamental group-theoretic presentation" of the Frobenioid-theoretic rational functions under consideration, in fact:

These Kummer classes still depend on a crucial piece of Frobenioidtheoretic data - namely, the cyclotome " $\boldsymbol{\mu}_{N}(-)$ ".

Of course, if one is only interested in this cyclotome up to isomorphism [i.e., up to multiplication by an element of $\left.(\mathbb{Z} / N \mathbb{Z})^{\times}\right]$, then the cyclotome may be 
thought of as being reconstuctible from the cyclotomic character of $\underline{\Pi}_{X}^{\mathrm{tp}}[\mathrm{cf}$., e.g., [Mzk2], Proposition 1.2.1, (vi)]. On the other hand, working with the coefficient cyclotome up to isomorphism amounts, in effect, [at the level of rational functions] to working with rational functions up to $\widehat{\mathbb{Z}}^{\times}$-powers - which is typically unacceptable in applications [e.g., where one wants, for instance, to specify the theta function, not the theta function up to $\widehat{\mathbb{Z}}^{\times}$-powers!]. This technical issue of "rigidity of the Frobenioid-theoretic cyclotome" is, in fact, a central theme of bi-Kummer theory and will be discussed further in $\S 5$ below.

\section{$\S 5 . \quad$ The Étale Theta Function via Tempered Frobenioids}

In the present $\S 5$, we carry out the goal of translating the scheme-theoretic constructions of $\S 1$ into the language of Frobenioids, by applying the general bi-Kummer theory of $\S 4$. This translation yields, in particular, a Frobenioidtheoretic construction of the mono-theta environments of $\S 2$ [cf. Theorem 5.10, (iii)].

In the following discussion, we return to the situation of Example 3.9. Suppose further that the morphism $\alpha: A \rightarrow B$ of Example 3.9, (iv), is the identity morphism, and that " $A$ " is one of the smooth log orbicurves

$$
\underline{X}^{\log } ; \quad \underline{C}^{\log } ; \quad \underline{\underline{X}}^{\log } ; \quad \underline{\underline{C}}^{\log } ; \quad \underline{\dot{X}}^{\log } ; \quad \underline{\dot{C}}^{\log } ; \quad \underline{\underline{X}}^{\log } ; \quad \underline{\underline{\dot{C}}}^{\log }
$$

[each of which is geometrically connected over the field $K=\ddot{K}$ ] of Definition 2.5 , (i), (ii), for some odd integer $l \geq 1$. We shall refer to the case where " $A$ " is $\underline{X}^{\log }, \underline{C}^{\log }, \underline{\dot{X}}^{\log }$, or $\underline{\dot{C}}^{\log }$ as the single underline case and to the case where " $A$ " is $\underline{\underline{X}}^{\log }, \underline{\underline{C}}^{\log }, \underline{\underline{X}}^{\log }$, or $\underline{\underline{\dot{C}}}^{\log }$ as the double underline case. Now the divisor monoid $\Phi \stackrel{\text { def }}{=} \Phi_{\alpha}^{\text {ell }}$ on $\mathcal{D} \stackrel{\text { def }}{=} \mathcal{D}_{\alpha}$ [cf. Example 3.9] determines a tempered Frobenioid

$\mathcal{C}$

of monoid type $\mathbb{Z}$ over the base category $\mathcal{D}$. We would like to apply the theory of $\S 4$ in the present situation. Thus, in the single underline (respectively, double underline) case, we take the object

$$
A_{\odot}
$$

of the theory of $\S 4$ to be the [Frobenius-trivial] object defined by the trivial line bundle over the object $\ddot{Y}^{\log }$ (respectively, $\underline{\underline{Y}}^{\log }$ ) of the discussion preceding Definition 2.7. Observe that this $A_{\odot}^{\text {bs }}$ is "characteristic" - that is to say, it 
is preserved by arbitrary self-equivalences of $\mathcal{D}$ [cf. Propositions 2.4, 2.6] hence, in particular, Galois. Let

$$
\Psi: \mathcal{C} \stackrel{\sim}{\rightarrow} \mathcal{C}
$$

be a self-equivalence of $\mathcal{C} ; N \geq 1$ an integer. Then it follows from Example 3.9, (iv), that we have the following:

Proposition 5.1 (Applicability of the General Theory). The Frobenioid $\mathcal{C}$ is a tempered Frobenioid of rationally standard type over a slim base category $\mathcal{D}$, whose monoid type is $\mathbb{Z}$, and whose divisor monoid $\Phi(-)$ is perfect, perf-factorial, non-dilating, and cuspidally pure. In particular, $\mathcal{C}$ and the self-equivalence $\Psi: \mathcal{C} \stackrel{\sim}{\rightarrow} \mathcal{C}$ satisfy all of the hypotheses of Corollary

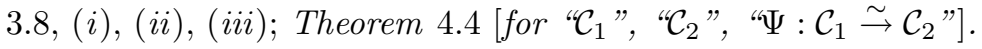

Remark 5.1.1. In Proposition 5.1, as well as in the discussion of the remainder of the present $\S 5$, we restrict our attention to self-equivalences, instead of considering arbitrary equivalences between possibly distinct categories, as in Theorem 4.4, or "functorial group-theoretic algorithms", as in Corollaries 2.18, 2.19. We do this, however, mainly to simplify the discussion [in particular, the notation]. That is to say, the extension to the case of arbitrary equivalences between possibly distinct categories satisfying similar hypotheses is, for the most part, immediate. Moreover, by sorting through the various arguments used in the proofs of the present paper, as well as in the proofs of the results of [Mzk17], [Mzk18], that are quoted, one concludes immediately the existence of "functorial category-theoretic algorithms", in the style of Corollaries 2.18, 2.19. We leave the routine clerical details to the interested reader.

Next, we reconsider the discussion of $\S 1$ [where we take the " $N$ " of $\S 1$ to be $l \cdot N]$ from the point of view of the theory of $\S 4$. To this end, let us first observe that by the definition of $\underline{\underline{Y}}^{\log }, \ddot{Z}_{l \cdot N}^{\log }$ [together with the fact that $l \cdot N$ is divisible by $l$, it follows immediately that the covering $\ddot{Z}_{l \cdot N}^{\log } \rightarrow X^{\log }$ factors through the covering $\underline{\underline{Y}}^{\log } \rightarrow X^{\log }$. Thus, if we pull-back the line bundle $\ddot{\mathfrak{L}}_{l \cdot N}$ on $\ddot{\mathfrak{Y}}_{l \cdot N}$ [cf. the discussion preceding Lemma 1.2] to $\ddot{\mathfrak{Z}}_{l \cdot N}$ [or, equivalently, the line bundle $\mathfrak{L}_{l \cdot N}$ on $\mathfrak{Y}_{l \cdot N}$, first to $\mathfrak{Z}_{l \cdot N}$, and then to $\left.\ddot{\mathfrak{Z}}_{l \cdot N}\right]$ so as to obtain a line bundle $\left.\ddot{\mathfrak{L}}_{l \cdot N}\right|_{\ddot{\mathfrak{Z}}_{l \cdot N}}$ on $\ddot{\mathfrak{Z}}_{l \cdot N}$, then the pull-backs to $\ddot{\mathfrak{Z}}_{l \cdot N}$ of

(1) the section $s_{l \cdot N} \in \Gamma\left(\mathfrak{Z}_{l \cdot N},\left.\mathfrak{L}_{l \cdot N}\right|_{\mathfrak{Z}_{l \cdot N}}\right)$ of Proposition 1.1, (i);

(2) the theta trivialization $\tau_{l \cdot N} \in \Gamma\left(\ddot{\mathfrak{Y}}_{l \cdot N}, \ddot{\mathfrak{L}}_{l \cdot N}\right)$ of Lemma 1.2

may be interpreted as morphisms

$$
\mathbb{V}\left(\mathcal{O}_{\ddot{\mathfrak{Z}}_{l \cdot N}}\right) \rightarrow \mathbb{V}\left(\left.\ddot{\mathfrak{L}}_{l \cdot N}\right|_{\ddot{\mathfrak{Z}}_{l \cdot N}}\right)
$$


- i.e., as morphisms between objects of $\mathcal{C}$. Now we have the following:

Proposition 5.2 (Bi-Kummer Theory). In the notation of the above discussion:

(i) The pair of morphisms of $\mathcal{C}$ determined by "sl.N", " $\tau_{l \cdot N}$ " constitutes an $l \cdot N$-th root of a right fraction-pair [cf. Proposition 4.2, (iii)] of [the Frobenioid-theoretic version of the log-meromorphic function constituted by] the theta function $\ddot{\Theta}$ of Proposition 1.4, or, alternatively, an N-th root of a right fraction-pair [cf. Proposition 4.2, (iii)] of [the Frobenioid-theoretic version of the log-meromorphic function constituted by] an l-th root of the theta function $\ddot{\Theta}[c f$. Remark 4.3.2].

(ii) The group actions of Proposition 1.1, (ii); Lemma 1.2, arising from "s $s_{l \cdot N}$ ", " $\tau_{l \cdot N}$ ", respectively, are precisely the actions determined by the biKummer $l \cdot N$-th root $[c f$. Proposition $4.3,(i)]$ arising from the $l \cdot N$-th root of $(i)$.

(iii) The Kummer class determined by the bi-Kummer $l \cdot N$-th root of (ii) $[$ cf. Proposition $4.3,($ iii) $]$ corresponds precisely to the reduction modulo $l \cdot N$ of the class " $\ddot{\eta} \Theta$ " of Proposition 1.3 - i.e., to the "étale theta function" - relative to the natural isomorphism [cf. Remark 5.2 .1 below] between " $\mu_{l \cdot N}(-)$ " $[$ cf. Proposition $4.3,($ iii $)]$ and $\underline{\Delta}_{\Theta} \otimes(\mathbb{Z} / l \cdot N \mathbb{Z})[c f$. Proposition 1.3]. Similarly, the Kummer class determined by the bi-Kummer $N$-th root of the $N$-th root of (i) [cf. Proposition 4.3, (iii)] corresponds precisely to the reduction modulo $N$ of the class " $\because{ }^{\Theta}$ " of the discussion preceding Definition 2.7 - i.e., to an l-th root of the "êttale theta function" - relative to the natural isomorphism between " $\boldsymbol{\mu}_{N}(-)=l \cdot \boldsymbol{\mu}_{l \cdot N}(-)$ " and $\left(l \cdot \underline{\Delta}_{\Theta}\right) \otimes(\mathbb{Z} / N \mathbb{Z})$.

Proof. These assertions follow immediately from the definitions. In the case of assertion (i), we observe that the " $\left.l \cdot N, H_{\odot},\left.f\right|_{A_{l \cdot N}}\right)$-saturated-ness" condition of Proposition 4.2, (iii), follows immediately from the definition of the field $\ddot{J}_{l \cdot N}$ in $\S 1$.

Remark 5.2.1. The natural isomorphisms of Proposition 5.2, (iii), constitute a scheme-theoretic ingredient in the otherwise Frobenioid-theoretic formulation of Proposition 5.2 [cf. Remark 4.4.1]. The translation of this final scheme-theoretic ingredient into category theory is the topic of Proposition 5.5; Theorems 5.6, 5.7 below.

Next, we consider divisors. Recall that the special fiber of ":̈" may be described as an infinite chain of copies of the projective line, joined to one another 
at the points " 0 " an " $\infty$ " [cf., e.g., the discussion preceding Proposition 1.1]. In particular, there is a natural bijection [cf. the discussion at the beginning of $\S 1]$

$$
\operatorname{Prime}\left(\Phi\left(A_{\odot}\right)\right)^{\mathrm{ncsp}} \stackrel{\sim}{\rightarrow} \mathbb{Z}
$$

which is well-defined, up to the operations of translation by an element of $\mathbb{Z}$ and multiplication by \pm 1 on $\mathbb{Z}$. Moreover, there is a natural surjection

$$
\operatorname{Prime}\left(\Phi\left(A_{\odot}\right)\right)^{\mathrm{csp}} \rightarrow \operatorname{Prime}\left(\Phi\left(A_{\odot}\right)\right)^{\mathrm{ncsp}}
$$

[i.e., given by considering the "irreducible component of the special fiber that contains the cusp(s) determined by the cuspidal prime"]. Also, let us observe that since all the cusps of $\ddot{Y}^{\log }$ arise from $\ddot{K}$-rational points, it follows immediately that one has the following cuspidal version of Remark 3.8.2: If $\mathfrak{p}$, $\mathfrak{q}$ are cuspidal primes of $\Phi\left(A_{\odot}\right)$, then there is a natural isomorphism between "primary components"

$$
\Phi\left(A_{\odot}\right)_{\mathfrak{p}} \stackrel{\sim}{\rightarrow} \Phi\left(A_{\odot}\right)_{\mathfrak{q}}
$$

— determined by identifying the elements on each side that arise from [schemetheoretic] prime log-divisors.

Next, let us choose an isomorphism $\Psi\left(A_{\odot}\right) \stackrel{\sim}{\rightarrow} A_{\odot}$ of $\mathcal{C}$ [cf. Proposition 5.1; Theorem 4.4, (i)]. Let us write

$$
\Psi_{A_{\odot}}^{\Phi}: \Phi\left(A_{\odot}\right) \stackrel{\sim}{\rightarrow} \Phi\left(A_{\odot}\right)
$$

for the automorphism of the monoid $\Phi\left(A_{\odot}\right)$ obtained by composing the isomorphism of divisor monoids induced by $\Psi: \mathcal{C} \stackrel{\sim}{\rightarrow} \mathcal{C}$ [cf. Corollary 3.8, (iii); [Mzk17], Theorem 4.9] with the isomorphism $\Phi\left(\Psi\left(A_{\odot}\right)\right) \stackrel{\sim}{\rightarrow} \Phi\left(A_{\odot}\right)$ induced by the chosen isomorphism $\Psi\left(A_{\odot}\right) \stackrel{\sim}{\rightarrow} A_{\odot}$.

Proposition 5.3 (Category-theoreticity of the Geometry of Divisors). In the notation of the above discussion, $\Psi_{A_{\odot}}^{\Phi}$ preserves the following objects:

(i) non-cuspidal and cuspidal elements;

(ii) the natural isomorphisms between distinct non-cuspidal primary components of $\Phi\left(A_{\odot}\right)$ [cf. Remark 3.8.2];

(iii) the natural isomorphisms [described in the discussion above] between distinct cuspidal primary components of $\Phi\left(A_{\odot}\right)$;

(iv) the natural surjection Prime $\left(\Phi\left(A_{\odot}\right)\right)^{\mathrm{csp}} \rightarrow \operatorname{Prime}\left(\Phi\left(A_{\odot}\right)\right)^{\mathrm{ncsp}}$;

$(v)$ the natural bijection Prime $\left(\Phi\left(A_{\odot}\right)\right)^{\mathrm{ncsp}} \stackrel{\sim}{\rightarrow} \mathbb{Z}[$ up to translation by an element of $\mathbb{Z}$ and multiplication by \pm 1$]$; 
(vi) the $\operatorname{Aut}_{\mathcal{C}}\left(A_{\odot}\right)$-orbit of the divisor of zeroes and poles $\in \Phi\left(A_{\odot}\right)^{\mathrm{gp}}$ of [the Frobenioid-theoretic version of the log-meromorphic function constituted by] the theta function $\ddot{\Theta}$ of Proposition 1.4.

Proof. The preservation of (i) (respectively, (ii)) follows immediately from Corollary 3.8, (iii) (respectively, Remark 3.8.2). To verify the preservation of the remaining objects, it suffices to consider the well-known "intersection theory of divisors supported on the chain of copies of the projective line" that constitutes the special divisor of ":̈" as follows:

Let us refer to pairs of elements of $\Phi\left(A_{\odot}\right)^{\text {gp }}$ whose difference lies in the image of the birational function monoid of the Frobenioid $\mathcal{C}$ as linearly equivalent. Let us refer to an element $\in \Phi\left(A_{\odot}\right)^{\text {gp }}$ which is linearly equivalent to 0 as principal. Let us refer to as cuspidally minimal any cuspidal element of $\Phi\left(A_{\odot}\right)^{\mathrm{gp}}$ whose support [cf. [Mzk17], Definition 2.4, (i), (d)] is a finite set of minimal cardinality among the cardinalities of supports of cuspidal elements of $\Phi\left(A_{\odot}\right)^{\mathrm{gp}}$ which are linearly equivalent to the given element.

Now the preservation of (iv) follows by considering the support of primary cuspidal elements $a \in \Phi\left(A_{\odot}\right)$ such that, for some $b \in \Phi\left(A_{\odot}\right)^{\mathrm{csp}}$ which is coprime to $a$ [i.e., $a, b$ have disjoint supports], $b-a$ is cuspidally minimal and linearly equivalent to a primary non-cuspidal element $n \in \Phi\left(A_{\odot}\right)$. That is to say, [by the well-known intersection theory of divisors supported on the chain of copies of the projective line] in this situation, $n$ is linearly equivalent to some element $\in \Phi\left(A_{\odot}\right)^{\mathrm{gp}}$ of the form $n_{1}+n_{2}-a$, where $n_{1}, n_{2} \in \Phi\left(A_{\odot}\right)^{\text {csp }}$ are primary cuspidal elements that map, respectively, via the natural surjection of (iv) to the two non-cuspidal primes that are adjacent [in the "chain of copies of the projective line"] to the non-cuspidal prime determined by $n$. Moreover, relative to the isomorphisms of (iii), the multiplicities of $n_{1}, n_{2}$ are equal to each other as well as to half the multiplicity of $a$. Thus, the natural isomorphisms of (iii) may be obtained by applying the natural isomorphisms of (ii) to the " $n$ " that occur for various " $a$ "; the natural surjection of (iv) is obtained by mapping the prime determined by $a$ to the prime determined by $n$.

To verify the preservation of (v), it suffices to show that the relation of adjacency [in the "chain of copies of the projective line"] between elements $\mathfrak{p}, \mathfrak{q} \in$ $\Phi\left(A_{\odot}\right)^{\text {ncsp }}$ is preserved. But this follows [again from the well-known intersection theory of divisors supported on the chain of copies of the projective line] by observing that if $a \in \mathfrak{p}, b \in \mathfrak{q}$ correspond via the natural isomorphisms of (ii), then $\mathfrak{p}, \mathfrak{q}$ are adjacent (respectively, not adjacent) if and only if every cuspidally minimal $c \in \Phi\left(A_{\odot}\right)^{\mathrm{gp}}$ which is linearly equivalent to $a+b$ has support of cardinality 4 (respectively, 5 or 6 ). [Here, the numbers " 4 ", " 5 ", "6" correspond 
to the number of non-cuspidal primes that are either contained in or adjacent to a prime contained in the support of $a+b$.]

Finally, the preservation of (vi), at least up to $\mathbb{Q}_{>0}$-multiples, follows immediately by considering the principal divisors $\in \Phi\left(A_{\odot}\right)^{\mathrm{gp}}$ in light of the preservation of (i), (ii), (iii), and (v) [cf. the description of the divisor of zeroes and poles of $\ddot{\Theta}$ in Proposition 1.4, (i)]; to eliminate the indeterminacy with respect to $\mathbb{Q}_{>0}$-multiples, it suffices to consider the zero divisor [cf. Remark 3.8.2] of a generator of $\mathcal{O}^{\triangleright}\left(A_{\odot}\right) / \mathcal{O}^{\times}\left(A_{\odot}\right) \cong \mathbb{Z}_{\geq 0}$ [cf. Proposition 3.4, (ii)].

Next, let us recall the characteristic [cf. Propositions 2.4, 2.6] subquotients

$$
\underline{\Pi}_{X}^{\mathrm{tp}} \rightarrow\left(\underline{\Pi}_{X}^{\mathrm{tp}}\right)^{\Theta} ; \quad l \cdot \underline{\Delta}_{\Theta} \subseteq\left(\underline{\Pi}_{X}^{\mathrm{tp}}\right)^{\Theta}
$$

of the discussion at the beginning of $\S 1$. Thus, for $D \in \mathrm{Ob}(\mathcal{D})$, these subquotients determine subquotients

$$
\operatorname{Aut}_{\mathcal{D}}(D) \rightarrow \operatorname{Aut}_{\mathcal{D}}^{\Theta}(D) ; \quad\left(l \cdot \underline{\Delta}_{\Theta}\right)_{D} \subseteq \operatorname{Aut}_{\mathcal{D}}^{\Theta}(D)
$$

which are preserved by arbitrary self-equivalences of $\mathcal{D}$ [cf. Propositions 2.4, 2.6]. If $S \in \mathrm{Ob}(\mathcal{C})$, then we shall write $\left(l \cdot \underline{\Delta}_{\Theta}\right)_{S} \stackrel{\text { def }}{=}\left(l \cdot \underline{\Delta}_{\Theta}\right)_{S^{\text {bs }}}$.

Definition 5.4. We shall say that $S \in \mathrm{Ob}(\mathcal{C})$ is $(l, N)$-theta-saturated if the following conditions are satisfied: (a) $S$ is $\boldsymbol{\mu}_{l \cdot N}$-saturated [cf. [Mzk18], Definition 2.1, (i)]; (b) $\left(l \cdot \underline{\Delta}_{\Theta}\right)_{S} \otimes \mathbb{Z} / N \mathbb{Z}$ is of cardinality $N$.

Now we may begin to address the issue discussed in Remark 5.2.1:

Proposition 5.5 (Frobenioid-theoretic Cyclotomic Rigidity). In the notation of the above discussion, the second Kummer class of Proposition 5.2 , (iii), determines an isomorphism

$$
\left(l \cdot \underline{\Delta}_{\Theta}\right)_{S} \otimes \mathbb{Z} / N \mathbb{Z} \stackrel{\sim}{\rightarrow} \boldsymbol{\mu}_{N}(S)\left(=l \cdot \boldsymbol{\mu}_{l \cdot N}(S)\right)
$$

for all $(l, N)$-theta-saturated $S \in \mathrm{Ob}(\mathcal{C})$. This isomorphism is functorial with respect to the subcategory of $\mathcal{C}$ determined by the linear morphisms of $(l, N)$-theta-saturated objects.

Proof. Indeed, if $S^{\prime} \in \mathrm{Ob}(\mathcal{C})$ is an $l \cdot N$-codomain of an $l \cdot N$-th root of a right fraction-pair of $\ddot{\Theta}$ [i.e., as discussed in Proposition 5.2, (i)], then it follows from the detailed description of the "étale theta class" in Proposition 1.3 that the resulting Kummer class [cf. Proposition 5.2, (iii)] determines an isomorphism

$$
\left(l \cdot \underline{\Delta}_{\Theta}\right)_{S^{\prime}} \otimes \mathbb{Z} / N \mathbb{Z} \stackrel{\sim}{\rightarrow} \boldsymbol{\mu}_{N}\left(S^{\prime}\right)
$$


which is manifestly "functorial" [in the evident sense, with respect to $l \cdot N$ codomains of $l \cdot N$-th roots of right fraction-pairs of $\Theta$ ] . Thus, we may transport this isomorphism from $S^{\prime}$ to an arbitrary $(l, N)$-theta-saturated $S \in \mathrm{Ob}(\mathcal{C})$ by means of linear morphisms $S^{\prime \prime} \rightarrow S, S^{\prime \prime} \rightarrow S^{\prime}$ [of $\mathcal{C}$ - cf. [Mzk17], Definition 1.3 , (i), (b)], which induce isomorphisms

$$
\begin{aligned}
& \left(l \cdot \underline{\Delta}_{\Theta}\right)_{S^{\prime \prime}} \otimes \mathbb{Z} / N \mathbb{Z} \stackrel{\sim}{\rightarrow}\left(l \cdot \underline{\Delta}_{\Theta}\right)_{S} \otimes \mathbb{Z} / N \mathbb{Z} ; \\
& \left(l \cdot \underline{\Delta}_{\Theta}\right)_{S^{\prime \prime}} \otimes \mathbb{Z} / N \mathbb{Z} \stackrel{\sim}{\rightarrow}\left(l \cdot \underline{\Delta}_{\Theta}\right)_{S^{\prime}} \otimes \mathbb{Z} / N \mathbb{Z} ; \\
& \boldsymbol{\mu}_{N}(S) \stackrel{\sim}{\rightarrow} \boldsymbol{\mu}_{N}\left(S^{\prime \prime}\right) ; \quad \boldsymbol{\mu}_{N}\left(S^{\prime}\right) \stackrel{\sim}{\rightarrow} \boldsymbol{\mu}_{N}\left(S^{\prime \prime}\right)
\end{aligned}
$$

- hence also a [functorial $]$ isomorphism $\left(l \cdot \underline{\Delta}_{\Theta}\right)_{S} \otimes \mathbb{Z} / N \mathbb{Z} \stackrel{\sim}{\rightarrow} \boldsymbol{\mu}_{N}(S)$, which is independent of the choice of $S^{\prime}, S^{\prime \prime}$ and the linear morphisms $S^{\prime \prime} \rightarrow S$, $S^{\prime \prime} \rightarrow S^{\prime}$ [precisely because of the original "functoriality" of the isomorphism for $\left.S^{\prime}\right]$.

Theorem 5.6 (Category-theoreticity of Frobenioid-theoretic Cyclotomic Rigidity). Write $\mathcal{C}$ for the tempered Frobenioid of monoid type $\mathbb{Z}$ determined by the divisor monoid $\Phi \stackrel{\text { def }}{=} \Phi_{\alpha}^{\text {ell }}$ on the base category $\mathcal{D} \stackrel{\text { def }}{=} \mathcal{D}_{\alpha}$ of Example 3.9, (iv), where we take the morphism $\alpha: A \rightarrow B$ of Example 3.9, (iv), to be the identity morphism and "A" to be one of the smooth log orbicurves

$$
\underline{X}^{\log } ; \quad \underline{C}^{\log } ; \quad \underline{\underline{X}}^{\log } ; \quad \underline{\underline{C}}^{\log } ; \quad \underline{\dot{X}}^{\log } ; \quad \underline{\dot{C}}^{\log } ; \quad \underline{\underline{X}}^{\log } ; \quad \underline{\underline{\dot{C}}}^{\log }
$$

[each of which is geometrically connected over the field $K=\ddot{K}$ ] of Definition $2.5,(i),(i i)$, for some odd integer $l \geq 1$. Let

$$
\Psi: \mathcal{C} \stackrel{\sim}{\rightarrow} \mathcal{C}
$$

be a self-equivalence of $\mathcal{C} ; N \geq 1$ an integer. Then $\Psi$ preserves the $(l, N)$ theta-saturated objects, as well as the natural isomorphism

$$
\left(l \cdot \underline{\Delta}_{\Theta}\right)_{S} \otimes \mathbb{Z} / N \mathbb{Z} \stackrel{\sim}{\rightarrow} \boldsymbol{\mu}_{N}(S)\left(=l \cdot \boldsymbol{\mu}_{l \cdot N}(S)\right)
$$

$[$ for $(l, N)$-theta-saturated $S \in \mathrm{Ob}(\mathcal{C})]$ of Proposition $5.5[$ [i.e., $\Psi$ transports this isomorphism for $S$ to the corresponding isomorphism for $\Psi(S)]$.

Proof. Indeed, by Proposition 5.1; [Mzk17], Theorem 3.4, (iv), (v), it follows that $\Psi$ preserves " $\mathcal{O}^{\times}(-)$" and induces a 1-compatible equivalence $\Psi^{\text {bs }}$ : $\mathcal{D} \stackrel{\sim}{\rightarrow} \mathcal{D}$, hence, [cf. [Mzk14], Proposition 3.2] an outer automorphism of the tempered fundamental group of the smooth orbicurve in question. In particular, 
it follows from Propositions $2.4,2.6$ that $\Psi$ preserves " $\left.\left(l \cdot \underline{\Delta}_{\Theta}\right)_{(-)}\right)$. Thus, it follows immediately that $\Psi$ preserves the $(l, N)$-theta-saturated objects.

Next, let $S_{2} \in \mathrm{Ob}(\mathcal{C})$ be an $(l, N)$-theta-saturated $l \cdot N$-codomain of an $l \cdot N$-th root of a right fraction-pair of $\ddot{\Theta}$ [i.e., as discussed in Proposition 5.2, (i)] such that $S_{2}^{\text {bs }}$ is "characteristic" [i.e., its isomorphism class is preserved by arbitrary self-equivalences of $\mathcal{D}$ ]; write

$$
s^{\sqcap}, s^{\sqcup}: S_{1} \rightarrow S_{2}
$$

for the pair of base-equivalent pre-steps that appear in this $l \cdot N$-th root [so $S_{1}$ is Frobenius-trivial]. Now since $\Psi$ preserves pre-steps and Frobenius-trivial objects and induces a 1-compatible self-equivalence $\Psi^{\text {bs }}: \mathcal{D} \stackrel{\sim}{\rightarrow} \mathcal{D}$ [cf. the proof of Theorem 4.4], it follows that $\Psi$ maps this pair of base-equivalent pre-steps to a pair of base-equivalent pre-steps

$$
t^{\sqcap}, t^{\sqcup}: T_{1} \rightarrow T_{2}
$$

such that $T_{1}$ is Frobenius-trivial, hence isomorphic to $S_{1}$ [cf. our assumption that $S_{1}^{\mathrm{bs}} \cong S_{2}^{\mathrm{bs}}$ is characteristic; [Mzk17], Theorem 5.1, (iii)] . Moreover, since $\Psi$ [essentially] preserves the divisor of zeroes and poles of $\ddot{\Theta}$ [cf. Proposition 5.3 , (vi)], it follows [cf. the equivalences of categories involving pre-steps of [Mzk17], Definition 1.3, (iii), (d)] that there exist isomorphisms $\gamma_{1}: S_{1} \stackrel{\sim}{\rightarrow} T_{1}$, $\gamma_{2}: S_{2} \stackrel{\sim}{\rightarrow} T_{2}, u \in \mathcal{O}^{\times}\left(T_{2}\right)$ such that $\gamma_{2} \circ s^{\sqcap}=t \sqcap \circ \gamma_{1}, u \circ \gamma_{2} \circ s^{\sqcup}=t^{\sqcup} \circ \gamma_{1}$. Thus, by forming the resulting group homomorphisms

$$
s^{\sqcap-\mathrm{gp}}, s^{\sqcup-\mathrm{gp}}: H_{S_{2}} \rightarrow \operatorname{Aut}_{\mathcal{C}}\left(S_{2}\right) ; \quad t^{\sqcap-\mathrm{gp}}, t^{\sqcup-\mathrm{gp}}: H_{T_{2}} \rightarrow \operatorname{Aut}_{\mathcal{C}}\left(T_{2}\right)
$$

[cf. Proposition 4.3, (i)] and Kummer classes [cf. Proposition 4.3, (iii)], and observing that the Kummer class of the "constant function" $u$ does not affect the restriction of the resulting Kummer classes to $\left(l \cdot \underline{\Delta}_{\Theta}\right)_{S_{2}},\left(l \cdot \underline{\Delta}_{\Theta}\right)_{T_{2}}$, we conclude that $\Psi$ does indeed transport the natural isomorphism $\left(l \cdot \underline{\Delta}_{\Theta}\right)_{S_{2}} \otimes$ $\mathbb{Z} / N \mathbb{Z} \stackrel{\sim}{\rightarrow} \boldsymbol{\mu}_{N}\left(S_{2}\right)$ of Proposition 5.5 to the corresponding isomorphism for $T_{2}$. Moreover, by the construction applied in the proof of Proposition 5.5, this preservation of the natural isomorphism of Proposition 5.5 in the specific case of $S_{2}$ is sufficient to imply the preservation of the natural isomorphism of Proposition 5.5 for arbitrary $(l, N)$-theta-saturated $S \in \mathrm{Ob}(\mathcal{C})$. This completes the proof of Theorem 5.6.

Theorem 5.7 (Category-theoreticity of the Frobenioid-theoretic Theta Function). In the notation of Theorem 5.6, $\Psi$ preserves right fraction- 
pairs of [the Frobenioid-theoretic version of the log-meromorphic function constituted by] an l-th root of the theta function

$$
\ddot{\Theta}(\sqrt{-1})^{-1} \cdot \ddot{\Theta}
$$

of Proposition 1.4 [normalized so as to be "of standard type"], up to possible multiplication by a $2 l$-th root of unity and possible translation by an element of $\underline{\mathbb{Z}}(\cong \operatorname{Gal}(\ddot{Y} / \dot{X}))\left[\right.$ when "A" arises from $\left.\underline{X}^{\log }, \underline{\dot{X}}^{\log }\right]$ or $l \cdot \underline{\mathbb{Z}}[$ when "A" arises from $\left.\underline{\underline{C}}^{\log }, \underline{\dot{X}}^{\log }, \underline{\underline{X}}^{\log }, \underline{\underline{X}}^{\log }, \underline{\underline{C}}^{\log }, \underline{\underline{\dot{C}}}^{\log }\right]$.

Proof. Indeed, this follows immediately by considering compatible systems as in Remark 4.3.2 and applying the theory of the rigidity of the étale theta function [cf. Corollary 2.8, (i)], to the Kummer classes of Proposition 5.2, (iii) [cf. also Theorem 4.4, (iii); Proposition 5.3, (vi)], above, in light of the crucial isomorphisms $\left(l \cdot \underline{\Delta}_{\Theta}\right)_{S} \otimes \mathbb{Z} / N \mathbb{Z} \stackrel{\sim}{\rightarrow} \boldsymbol{\mu}_{N}(S)$ of Proposition 5.5, which, by Theorem 5.6, are preserved by $\Psi$.

Remark 5.7.1. Note that the "rigidity up to possible multiplication by a $2 l$-th root of unity" asserted in Theorem 5.7 is substantially stronger than [what was in effect] the preservation of $\ddot{\Theta}$ up to multiplication by an arbitrary constant function $\in \mathcal{O}^{\times}(-)$[cf. the " $u$ " appearing in the proof of Theorem 5.6], that was observed by considering divisors [i.e., Proposition 5.3, (vi)] in the proof of Theorem 5.6.

Next, we consider theta environments. For the remainder of the present $\S 5$, we suppose that:

$$
\text { "A" arises from } \underline{\underline{X}}^{\log }
$$

Write

$$
s_{N}^{\sqcap}, s_{N}^{\sqcup}: A_{N} \rightarrow B_{N}
$$

for the pair of base-equivalent morphisms of $\mathcal{C}$ determined by the sections " $s_{l \cdot N}$ ", " $\tau_{l \cdot N}$ " of the discussion preceding Proposition 5.2 [so $s_{N}^{\sqcap}, s_{N}^{\sqcup}$ constitute an $N$-th root of a right fraction-pair of an $l$-th root of the theta function $\ddot{\Theta}$ - cf. Proposition 5.2, (i)]. Note that the zero divisor $\operatorname{Div}\left(s_{N}^{\sqcap}\right) \in \Phi\left(A_{N}\right)$ of $s_{N}^{\sqcap}$ descends [relative to the unique morphism $A_{\odot}^{\text {bs }} \rightarrow A$ in $\mathcal{D}$ ] to $\Phi(A)$ [cf. Proposition 1.4, (i)]; in particular, it follows that $B_{N}$ is Aut-ample. Thus, the group homomorphism $s_{N}^{\text {trv }}: \operatorname{Aut}_{\mathcal{D}}\left(A_{N}^{\text {bs }}\right) \rightarrow \operatorname{Aut}_{\mathcal{C}}\left(A_{N}\right)$ arising from a base-Frobenius pair of $A_{N}$ [cf. Proposition 5.1; Theorem 3.7, (i); [Mzk17], Proposition 5.6], 
which is completely determined [cf. Proposition 5.1; Theorem 3.7, (i); [Mzk17], Proposition 5.6] up to conjugation by an element of $\mathcal{O}^{\times}\left(A_{N}\right)$, determines unique group homomorphisms

$$
s_{N}^{\sqcap \text {-gp }}: \operatorname{Aut}_{\mathcal{D}}\left(B_{N}^{\mathrm{bs}}\right) \rightarrow \operatorname{Aut}_{\mathcal{C}}\left(B_{N}\right) ; \quad s_{N}^{\sqcup-\mathrm{gp}}: H_{B_{N}} \rightarrow \operatorname{Aut}_{\mathcal{C}}\left(B_{N}\right)
$$

such that, relative to the isomorphisms $\operatorname{Aut}_{\mathcal{D}}\left(A_{N}^{\text {bs }}\right) \stackrel{\sim}{\rightarrow} \operatorname{Aut}_{\mathcal{D}}\left(B_{N}^{\text {bs }}\right), H_{A_{N}} \stackrel{\sim}{\rightarrow} H_{B_{N}}$ determined by the [base-equivalent!] pair of morphisms $s_{N}^{\sqcap}, s_{N}^{\sqcup}$, we have

$$
s_{N}^{\sqcap-\mathrm{gp}}(g) \circ s_{N}^{\sqcap}=s_{N}^{\sqcap} \circ\left(\left.s_{N}^{\mathrm{trv}}\right|_{\operatorname{Aut}_{\mathcal{D}}\left(B_{N}^{\mathrm{bs}}\right)}\right)(g) ; \quad s_{N}^{\sqcup-\mathrm{gp}}(h) \circ s_{N}^{\sqcup}=s_{N}^{\sqcup} \circ\left(\left.s_{N}^{\mathrm{trv}}\right|_{H_{B_{N}}}\right)(h)
$$

for all $g \in \operatorname{Aut}_{\mathcal{D}}\left(B_{N}^{\text {bs }}\right), h \in H_{B_{N}}$ [cf. Propositions 4.3, (i); 5.2, (ii)]. Write

$$
\mathbb{E}_{N} \stackrel{\text { def }}{=} s_{N}^{\sqcap-g p}\left(\operatorname{Im}\left(\underline{\Pi}_{\underline{\underline{Y}}}^{\mathrm{tp}}\right)\right) \cdot \boldsymbol{\mu}_{N}\left(B_{N}\right) \subseteq \operatorname{Aut}_{\mathcal{C}}\left(B_{N}\right)
$$

- where $\operatorname{Im}\left(\underline{\Pi}_{\underline{Y}}^{\mathrm{tp}}\right)$ denotes the image of $\underline{\Pi}_{\underline{\underline{Y}}}^{\mathrm{tp}} \subseteq \underline{\Pi}_{\underline{X}}^{\mathrm{tp}}$ via the natural outer homomorphism $\underline{\Pi}_{\underline{\underline{X}}}^{\text {tp }} \rightarrow \operatorname{Aut}_{\mathcal{D}}\left(B_{N}^{\text {bs }}\right)$ [cf. Definition 4.1, (ii)].

Lemma 5.8 (Conjugation by Constants). In the notation of the above discussion, write

$$
\left(K^{\times}\right)^{1 / N} \subseteq \mathcal{O}^{\times}\left(B_{N}^{\text {birat }}\right)
$$

for the subgroup of elements whose $N$-th power lies in the image of the natural inclusion $K^{\times} \hookrightarrow \mathcal{O}^{\times}\left(B_{N}^{\text {birat }}\right) ;\left(\mathcal{O}_{K}^{\times}\right)^{1 / N} \stackrel{\text { def }}{=}\left(K^{\times}\right)^{1 / N} \cap \mathcal{O}^{\times}\left(B_{N}\right)$. Then $\left(\mathcal{O}_{K}^{\times}\right)^{1 / N}$ is equal to the set of elements of $\mathcal{O}^{\times}\left(B_{N}\right)$ that normalize the subgroup $\mathbb{E}_{N} \subseteq \operatorname{Aut}_{\mathcal{C}}\left(B_{N}\right)$. In particular, we have a natural outer action of

$$
\left(\mathcal{O}_{K}^{\times}\right)^{1 / N} / \boldsymbol{\mu}_{N}\left(B_{N}\right) \stackrel{\sim}{\rightarrow} \mathcal{O}_{K}^{\times}
$$

on $\mathbb{E}_{N} ;$ this outer action extends to an outer action of $\left(K^{\times}\right)^{1 / N} / \boldsymbol{\mu}_{N}\left(B_{N}\right) \stackrel{\sim}{\rightarrow}$ $K^{\times}$on $\mathbb{E}_{N}$.

Proof. Indeed, since $\underline{\underline{Y}}$ is geometrically connected over $K$, it follows immediately that the set of elements of $\mathcal{O}^{\times}\left(B_{N}\right)$ that normalize the subgroup $\mathbb{E}_{N} \subseteq \operatorname{Aut}_{\mathcal{C}}\left(B_{N}\right)$ is equal to the set of elements on which $\underline{\underline{\underline{Y}}}_{\underline{\underline{\underline{p}}}}^{\mathrm{tp}}$ [i.e., $G_{K}$, via the natural surjection $\left.\underline{\Pi}_{\underline{\underline{Y}}}^{\mathrm{tp}} \rightarrow G_{K}\right]$ acts via multiplication by an element of $\boldsymbol{\mu}_{N}\left(B_{N}\right)$. But this last set is easily seen to coincide with $\left(\mathcal{O}_{K}^{\times}\right)^{1 / N}$.

Lemma 5.9 (First Properties of Frobenioid-theoretic Theta Environments). In the notation of the above discussion:

(i) $\left.s_{N}^{\sqcap \text {-gp }}\right|_{H_{B_{N}}}, s_{N}^{\sqcup \text {-gp }}$ factor through $\mathbb{E}_{N}$. 
(ii) We have a natural exact sequence

$$
1 \rightarrow \boldsymbol{\mu}_{N}\left(B_{N}\right) \rightarrow \mathbb{E}_{N} \rightarrow \operatorname{Im}\left(\underline{\underline{M}}_{\underline{\underline{Y}}}^{\mathrm{tp}}\right) \rightarrow 1
$$

- where $\operatorname{Im}\left(\underline{\Pi}_{\underline{\underline{Y}}}^{\mathrm{tp}}\right)$ denotes the image of $\underline{\underline{\Pi}}_{\underline{\underline{Y}}}^{\mathrm{tp}} \underline{\underline{\Pi_{\underline{X}}}} \underline{\underline{\underline{X}}}$ via the natural outer homomorphism $\underline{\underline{\underline{X}}} \underline{\underline{\underline{x}}} \rightarrow \operatorname{Aut}_{\mathcal{D}}\left(B_{N}^{\mathrm{bs}}\right)[c f$. Definition 4.1, $(i i)]$.

(iii) We have a natural outer action

$$
l \cdot \underline{\mathbb{Z}} \stackrel{\sim}{\rightarrow} \underline{\Pi}_{\underline{\underline{X}}}^{\mathrm{tp}} / \underline{\Pi}_{\underline{\underline{Y}}}^{\mathrm{tp}} \rightarrow \operatorname{Out}\left(\mathbb{E}_{N}\right)
$$

determined by conjugating via the composite of the natural outer homomorphism $\underline{\Pi}_{\underline{\underline{X}}}^{\mathrm{tp}} \rightarrow \operatorname{Aut}_{\mathcal{D}}\left(B_{N}^{\mathrm{bs}}\right)$ with $s_{N}^{\Pi \text {-gp }}: \operatorname{Aut}_{\mathcal{D}}\left(B_{N}^{\mathrm{bs}}\right) \rightarrow \operatorname{Aut}_{\mathcal{C}}\left(B_{N}\right)$.

(iv) Write

$$
\mathbb{E}_{N}^{\Pi} \stackrel{\text { def }}{=} \mathbb{E}_{N} \times_{\operatorname{Im}\left(\underline{\Pi}_{\underline{\underline{\underline{I}}}}^{\mathrm{tp}}\right)} \underline{\underline{\underline{Y}}}_{\underline{\underline{\mathrm{tp}}}}^{\mathrm{tp}}
$$

- where $\operatorname{Im}\left(\underline{\Pi}_{\underline{Y}}^{\mathrm{tp}}\right)$ is as in $(i i) ; \mathbb{E}_{N}, \mathbb{E}_{N}^{\Pi}$ are equipped with the evident topologies; the homomorphism $\underline{\underline{\Pi}}_{\underline{\underline{Y}}}^{\mathrm{tp}} \rightarrow \operatorname{Im}\left(\underline{\Pi}_{\underline{\underline{Y}}}^{\mathrm{tp}}\right)$ is well-defined up to conjugation by an element of $\underline{\Pi}_{\underline{X}}^{\mathrm{tp}}$. Then the natural inclusions $\boldsymbol{\mu}_{N}\left(B_{N}\right) \hookrightarrow \mathbb{E}_{N}, \operatorname{Im}\left(\underline{\Pi}_{\underline{Y}}^{\mathrm{tp}}\right) \subseteq \mathbb{E}_{N}$ determine an isomorphism of topological groups

$$
\mathbb{E}_{N}^{\Pi} \stackrel{\sim}{\rightarrow} \underline{\underline{M}}_{\underline{\underline{Y}}}^{\mathrm{tp}}\left[\boldsymbol{\mu}_{N}\right]
$$

which is an isomorphism of $\bmod N$ bi-theta environments with respect to the model bi-theta environment structure of Definition $2.13,($ ii $)$, on $\underline{\Pi}_{\underline{\underline{Y}}}^{\mathrm{tp}}\left[\boldsymbol{\mu}_{N}\right]$ and the $\bmod N$ bi-theta environment structure on $\mathbb{E}_{N}^{\Pi}$ determined by the subgroup of $\operatorname{Out}\left(\mathbb{E}_{N}^{\Pi}\right)$ generated by the natural outer actions of $l \cdot \underline{\mathbb{Z}}[$ cf. (iii) $], K^{\times}$ [cf. Lemma 5.8] on $\mathbb{E}_{N}$, together with the $\boldsymbol{\mu}_{N}$-conjugacy classes of subgroups given by the images of the homomorphisms

$$
s_{N}^{\sqcap-\Pi}, s_{N}^{\sqcup-\Pi}: \underline{\Pi}_{\underline{\underline{\underline{Y}}}}^{\mathrm{tp}} \rightarrow \mathbb{E}_{N}^{\Pi}
$$

arising from $s_{N}^{\sqcap \text {-gp }}, s_{N}^{\sqcup \text {-gp }}[c f .(i)]$. In particular, omitting the homomorphism $s_{N}^{\Pi-\Pi}$ yields a $\bmod N$ mono-theta environment.

$(v)$ In the situation of $(i v)$, the cyclotomic rigidity isomorphism arising from the theory of $\S 2[c f$. Corollary 2.19, $(i)]$ coincides with the Frobenioidtheoretic isomorphism of Proposition 5.5 [where we take " $S$ " to be $B_{N}$ ].

Proof. Immediate from the definitions [with regard to assertion (iii), cf. also Proposition 5.2, (ii)]. 
We are now ready to state our main result relating the theory of theta environments of $\S 2$ to the theory of tempered Frobenioids discussed in $\S 3, \S 4$, and the present $\S 5$.

Theorem 5.10 (Category-theoreticity of Frobenioid-theoretic Theta Environments). In the notation of Theorem 5.6, suppose further that " $A$ " arises from $\underline{\underline{X}}^{\log }$; write

$$
s_{N}^{\sqcap}, s_{N}^{\sqcup}: A_{N} \rightarrow B_{N}
$$

for the pair of base-equivalent morphisms of $\mathcal{C}$ determined by the sections " $s_{l \cdot N}$ ", " $\tau_{l \cdot N}$ " of the discussion preceding Proposition $5.2-s o s_{N}^{\sqcap}, s_{N}^{\sqcup}$ constitute an $N$-th root of a right fraction-pair of an l-th root of the theta function $\ddot{\Theta}$ [cf. Proposition 5.2, (i)];

$$
\mathbb{E}_{N} \subseteq \operatorname{Aut}_{\mathcal{C}}\left(B_{N}\right)
$$

for the subgroup defined in the discussion preceding Lemma 5.8;

$$
\mathbb{E}_{N}^{\Pi \stackrel{\text { def }}{=}} \mathbb{E}_{N} \times_{\operatorname{Im}\left(\underline{\Pi}_{\underline{\underline{Y}}}^{\mathrm{tp}}\right)} \underline{\Pi}_{\underline{\underline{Y}}}^{\mathrm{tp}}
$$

for the topological group defined in Lemma 5.9, (iv);

$$
\epsilon: \mathbb{E}_{N}^{\Pi} \rightarrow \operatorname{Aut}_{\mathcal{C}}\left(B_{N}\right)
$$

for the $\boldsymbol{\mu}_{N}$-outer homomorphism [i.e., homomorphism considered up to composition with an inner automorphism defined by an element of $\operatorname{Ker}\left(\mathbb{E}_{N}^{\Pi} \rightarrow\right.$ $\left.\underline{\Pi}_{Y}^{\mathrm{tp}}\right)$ or $\left.\boldsymbol{\mu}_{N}\left(B_{N}\right) \subseteq \operatorname{Aut}_{\mathcal{C}}\left(B_{N}\right)\right]$ determined by the natural projection $\mathbb{E}_{N}^{\Pi} \rightarrow$ $\mathbb{E}_{N}\left(\subseteq \operatorname{Aut}_{\mathcal{C}}\left(B_{N}\right)\right)$. Then:

(i) The self-equivalence $\Psi: \mathcal{C} \stackrel{\sim}{\rightarrow} \mathcal{C}$ preserves the isomorphism classes of $A_{N}, B_{N}$.

(ii) Let $\beta$ be an isomorphism $\beta: \Psi\left(B_{N}\right) \stackrel{\sim}{\rightarrow} B_{N}[c f .(i)]$; write

$\Psi_{\text {Aut }}: \operatorname{Aut}_{\mathcal{C}}\left(B_{N}\right) \stackrel{\sim}{\rightarrow} \operatorname{Aut}_{\mathcal{C}}\left(B_{N}\right) ; \quad \Psi_{\text {Aut }}^{\text {birat }}: \operatorname{Aut}_{\mathcal{C}^{\text {birat }}}\left(B_{N}^{\text {birat }}\right) \stackrel{\sim}{\rightarrow} \operatorname{Aut}_{\mathcal{C}^{\text {birat }}}\left(B_{N}^{\text {birat }}\right)$

for the automorphisms determined by applying $\Psi$ followed by conjugation by $\beta$. Then $\Psi_{\text {Aut }}, \Psi_{\text {Aut }}^{\text {birat }}$ preserve

$$
\mathcal{O}^{\times}\left(B_{N}\right) ; \quad\left(\mathcal{O}_{K}^{\times}\right)^{1 / N} \subseteq \mathcal{O}^{\times}\left(B_{N}\right) ; \quad \mathcal{O}^{\times}\left(B_{N}^{\text {birat }}\right) ; \quad\left(K^{\times}\right)^{1 / N} \subseteq \mathcal{O}^{\times}\left(B_{N}^{\text {birat }}\right)
$$

and map the data

$$
\mathbb{E}_{N}\left(\subseteq \operatorname{Aut}_{\mathcal{C}}\left(B_{N}\right)\right) ; \quad \operatorname{Im}\left(s_{N}^{\sqcap-\mathrm{gp}}\right) ; \quad \operatorname{Im}\left(s_{N}^{\sqcup-\mathrm{gp}}\right)
$$


- where $\operatorname{Im}(-)$ " denotes the image of the homomorphism in parentheses to data

$$
\begin{gathered}
\delta_{1} \cdot \mathbb{E}_{N} \cdot \delta_{1}^{-1}\left(\subseteq \operatorname{Aut}_{\mathcal{C}}\left(B_{N}\right)\right) ; \quad \delta_{1} \cdot \operatorname{Im}\left(s_{N}^{\sqcap-\mathrm{gp}}\right) \cdot \delta_{1}^{-1} ; \\
\delta_{1} \cdot \delta_{2} \cdot \delta_{3} \cdot \operatorname{Im}\left(s_{N}^{\sqcup-\mathrm{gp}}\right) \cdot \delta_{3}^{-1} \cdot \delta_{2}^{-1} \cdot \delta_{1}^{-1}
\end{gathered}
$$

for some $\delta_{1} \in \mathcal{O}^{\times}\left(B_{N}\right)$, some $\delta_{2} \in \boldsymbol{\mu}_{2 l \cdot N}\left(B_{N}\right) \bigcap\left(\mathcal{O}_{K}^{\times}\right)^{1 / N}\left(\subseteq \mathcal{O}^{\times}\left(B_{N}\right)\right)$, and some $\delta_{3} \in s_{N}^{\sqcap-g p}\left(\operatorname{Aut}_{\mathcal{D}}\left(B_{N}^{\mathrm{bs}}\right)\right)$.

(iii) The operation of applying $\Psi$ followed by conjugation by $\beta$ preserves the $\operatorname{Aut}_{\mathcal{C}}\left(B_{N}\right)$-orbit of $\epsilon: \mathbb{E}_{N}^{\Pi} \rightarrow \operatorname{Aut}_{\mathcal{C}}\left(B_{N}\right)$, in a fashion which is compatible with the mono-theta environment structure on $\mathbb{E}_{N}^{\Pi}$ involving $s_{N}^{\sqcup-\Pi}$ discussed in Lemma 5.9, (iv). More precisely: there exists a commutative diagram

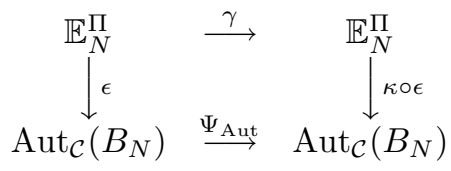

- where $\kappa$ is an inner automorphism of $\operatorname{Aut}_{\mathcal{C}}\left(B_{N}\right) ; \gamma$ is an automorphism of topological groups which determines an automorphism of mono-theta environments and is compatible with the $\underline{\Pi}_{\underline{X}}^{\text {tp }}$-conjugacy class of automorphisms of $\underline{\Pi}_{\underline{\underline{Y}}}^{\mathrm{tp}}$ induced by $\Psi^{\mathrm{bs}}[$ cf. Theorem 4.4].

Proof. First, we observe that by Proposition 5.1, the hypotheses of Theorem 4.4 are satisfied. Next, we consider assertion (i). Since $\Psi$ preserves Frobenius-trivial objects [cf. the proof of Theorem 4.4], to show that $\Psi$ preserves the isomorphism class of $A_{N}$, it suffices to show that the equivalence $\Psi^{\text {bs }}: \mathcal{D} \stackrel{\sim}{\rightarrow} \mathcal{D}$ [cf. Theorem 4.4] induced by $\Psi$ preserves the isomorphism class of the objects of $\mathcal{D}$ determined by " $\ddot{Z}_{l \cdot N}^{\log }$ ", " $\ddot{\underline{Y}}^{\log }$ "; but this follows immediately [in light of the definitions of the various tempered coverings involved] from Proposition 2.4. Now the fact that $\Psi$ preserves the isomorphism class of $B_{N}$ follows immediately from Proposition 5.3, (vi). This completes the proof of assertion (i).

Next, we consider assertion (ii). First, we observe that it follows from the existence of $\Psi^{\mathrm{bs}}$ and the fact that $\Psi$ preserves pre-steps [cf. the proof of Theorem 4.4] that $\Psi$ preserves " $\mathcal{O}^{\triangleright}(-)$ ", as well as base-equivalent pairs of pre-steps. In particular, it follows that applying $\Psi$ followed by conjugation by $\beta$ preserves $\mathcal{O}^{\triangleright}\left(B_{N}\right), \mathcal{O}^{\times}\left(B_{N}\right), \mathcal{O}^{\times}\left(B_{N}^{\text {birat }}\right)$. Thus, [cf. Proposition 3.4, (ii)] we conclude [by considering Galois-, i.e., $\operatorname{Aut}_{\mathcal{C}}\left(B_{N}\right)$-invariants] that $\Psi_{\text {Aut }}^{\text {birat }}$ preserves the image of the natural inclusion $K^{\times} \hookrightarrow \mathcal{O}^{\times}\left(B_{N}^{\text {birat }}\right)$, hence also $\left(K^{\times}\right)^{1 / N},\left(\mathcal{O}_{K}^{\times}\right)^{1 / N}$. Finally, the portion of assertion (ii) concerning $\operatorname{Im}\left(s_{N}^{\sqcap-\mathrm{gp}}\right)$, $\operatorname{Im}\left(s_{N}^{\sqcup-g p}\right), \mathbb{E}_{N}$ follows by observing that $\Psi$ preserves $N$-th roots of fraction 
pairs [cf. Theorem 4.4, (ii)], together with the corresponding bi-Kummer $N$-th roots [cf. Theorem 4.4, (iv)], for [the Frobenioid-theoretic version of the logmeromorphic function constituted by] $l$-th roots of the theta function [i.e., up to the indeterminacies discussed in the statement of Theorem $5.7-\mathrm{cf}$. Theorem 5.7]. This completes the proof of assertion (ii).

Finally, we consider assertion (iii). First, observe that it follows from the existence of $\kappa$ that, in the following argument, we may treat $\epsilon$ as a single fixed homomorphism, rather than just a " $\boldsymbol{\mu}_{N}$-outer homomorphism". Next, we observe that by taking $\kappa$ to be the inner automorphism of $\operatorname{Aut}_{\mathcal{C}}\left(B_{N}\right)$ determined by conjugating by the element $\delta_{1} \cdot \delta_{2} \cdot \delta_{3} \in \operatorname{Aut}_{\mathcal{C}}\left(B_{N}\right)$ of assertion (ii), we may assume that the restrictions of $\Psi_{\text {Aut }} \circ \epsilon, \kappa \circ \epsilon$ to the image of $s_{N}^{\llcorner-\Pi}: \underline{\Pi}_{\underline{\underline{Y}}}^{\mathrm{tp}} \rightarrow \mathbb{E}_{N}^{\Pi}$ coincide, up to composition with an automorphism of the topological group $\underline{\Pi}_{\underline{\underline{\underline{Y}}}}^{\mathrm{tp}}$ that extends to an automorphism of the topological group $\underline{\underline{\Pi}}_{\underline{\underline{X}}}^{\mathrm{tp}}$ [which lies in the $\underline{\Pi}_{\underline{X}}^{\text {tp }}$-conjugacy class of automorphisms of $\underline{\Pi}_{\underline{\underline{Y}}}^{\mathrm{tp}}$ induced by $\left.\Psi^{\mathrm{bs}}\right]$. Thus, by applying Corollary 2.18, (iv), it follows that we may choose $\gamma$ so that the restrictions of $\Psi_{\mathrm{Aut}} \circ \epsilon, \kappa \circ \epsilon \circ \gamma$ to the image of $s_{N}^{\sqcup-\Pi}$ coincide [precisely]. Moreover, by applying the compatible [cf. Lemma 5.9, (v)], category/grouptheoretic [cf. Theorem 5.6; Corollary 2.19, (i)] cyclotomic rigidity isomorphisms of Proposition 5.5 and Corollary 2.19, (i) [cf. also Remark 2.19.3], it follows that the restrictions of $\Psi_{\text {Aut }} \circ \epsilon, \kappa \circ \epsilon \circ \gamma$ to $\operatorname{Ker}\left(\mathbb{E}_{N}^{\Pi} \rightarrow \underline{\Pi}_{\underline{\underline{Y}}}^{\mathrm{tp}}\right)$ coincide. Thus, we conclude that the restrictions of $\Psi_{\text {Aut }} \circ \epsilon, \kappa \circ \epsilon \circ \gamma$ to $\underline{\underline{M}}_{\underline{\underline{\underline{Y}}}}^{\mathrm{tp}}\left[\boldsymbol{\mu}_{N}\right] \subseteq \underline{\underline{\Pi}}_{\underline{\underline{Y}}}^{\mathrm{tp}}\left[\boldsymbol{\mu}_{N}\right] \stackrel{\sim}{\rightarrow} \mathbb{E}_{N}^{\Pi}$ [where we apply the isomorphism of Lemma 5.9, (iv)] coincide. Since $\left[\underline{\underline{\Pi}}_{\underline{\underline{Y}}}^{\mathrm{tp}}\right.$ : $\left.\underline{\underline{\Pi}}_{\underline{\underline{\underline{Y}}}}^{\mathrm{tp}}\right]=2$, it thus follows that the difference between $\Psi_{\text {Aut }} \circ \epsilon, \kappa \circ \epsilon \circ \gamma$ determines a cohomology class [cf. the proof of Corollary 2.18, (iv)]

$$
\in H^{1}\left(\underline{\underline{\Pi_{Y}}} / \underline{\underline{\Pi}}_{\underline{\underline{\underline{Y}}}}^{\mathrm{tp}}, \mathcal{O}^{\times}\left(B_{N}\right)^{G_{K}}\right) \cong H^{1}\left(\mathbb{Z} / 2 \mathbb{Z}, \mathcal{O}_{K}^{\times}\right) \cong \boldsymbol{\mu}_{2}\left(B_{N}\right)
$$

- where the superscript " $G_{K}$ " denotes the subgroup of $G_{K}$-invariants; we apply Proposition 3.4, (ii), and recall that $\ddot{K}=K$. Thus, by composing $\gamma$ with an appropriate order two automorphism of mono-theta environments [cf. Corollary 2.18, (iv), and its proof], we obtain a $\gamma$ such that $\Psi_{\text {Aut }} \circ \epsilon=\kappa \circ \epsilon \circ \gamma$, as desired. This completes the proof of assertion (iii).

Remark 5.10.1. Observe that Theorem 5.10, (iii), may be interpreted as asserting that a mono-theta environment may be "extracted" from the tempered Frobenioids under consideration in a purely category-theoretic fashion. In particular, by coupling this observation with Corollary 2.18 [cf. also Remark 2.18.2], we conclude that: 
A mono-theta environment may be "extracted" naturally from both the tempered Frobenioids and the tempered fundamental groups under consideration in a purely category/group-theoretic fashion.

That is to say, a mono-theta environment may be thought of as a sort of minimal core common to both the [tempered-] étale-theoretic and Frobenioid-theoretic approaches to the theta function [cf. Remark 2.18.2]. Put another way, from the point of view of the general theory of Frobenioids, although the "étale-like" [e.g., temperoid]" and "Frobenius-like" portions of a Frobenioid are fundamentally alien to one another in nature [cf. the "fundamental dichotomy" discussed in [Mzk17], Remark 3.1.3], a mono-theta environment serves as a sort of bridge, relative to the theory of theta function, between these two fundamentally mutually alien aspects of the structure of a Frobenioid.

Remark 5.10.2. The structure of a Frobenioid may be thought of as consisting of a sort of extension structure of the base category by various line bundles. From this point of view:

The theta section portion of a mono-theta environment may be thought as a sort of canonical splitting of this extension, determined by the theory of the étale theta function [cf. the discussion of canonicality/rigidity in the Introduction].

This point of view is reminiscent [cf. the point of view of Remark 5.10.1] of the notion of a "canonical uniformizing $\mathcal{M F}^{\nabla}$-object" discussed in [Mzk1], Introduction, $§ 1.3$.

Remark 5.10.3. One key feature of a mono-theta environment is the inclusion in the mono-theta environment of the "distinct cyclotome" $\boldsymbol{\mu}_{N}\left(B_{N}\right) \cong$ $\operatorname{Ker}\left(\mathbb{E}_{N}^{\Pi} \rightarrow \underline{\underline{M}}_{\underline{\underline{Y}}}^{\mathrm{tp}}\right)$ [i.e., a cyclotome distinct from the various cyclotomes associated to $\left.\underline{\underline{M}}_{\underline{\underline{Y}}}^{\mathrm{tp}}\right]$. Here, we pause to observe that:

This "distinct cyclotome" may be thought of as a sort of "Frobenius germ" - i.e., a "germ" or "trace" of the "Frobenius structure" of the tempered Frobenioid $\mathcal{C}$ constituted by raising elements of $\mathcal{O}^{\triangleright}(-)$ to $\mathbb{N}_{\geq 1 \text {-powers. }}$

Indeed, when a mono-theta environment is not considered as a separate, abstract mathematical structure, but rather as a mathematical structure associated to the tempered Frobenioid $\mathcal{C}$, the operation of "raising to $\mathbb{N}_{\geq 1}$-powers" elements of $\mathcal{O}^{\triangleright}(-)$ in $\mathcal{C}$ is compatible with the natural multiplication action 
of $\mathbb{N}_{>1}$ on this distinct cyclotome. Moreover, these actions of $\mathbb{N}_{>1}$ are compatible with the operation of forming Kummer classes [e.g., passing from the Frobenioid-theoretic version of the theta function to its Kummer class, the "étale theta function"], as well as with the consideration of values $\in K^{\times}$of functions [e.g., the theta function - cf. Proposition 1.4, (iii)], relative to the reciprocity map on elements of $K^{\times}$[cf. [Mzk18], Theorem 2.4]. On the other hand, it is important to note that:

These actions of $\mathbb{N}_{\geq 1}$ only make sense within the tempered Frobenioid $\mathcal{C}$; that is to say, they do not give rise to an action of $\mathbb{N}_{\geq 1}$ on the monotheta environment [considered as a separate, abstract mathematical structure].

Indeed, the fact that one does not obtain a natural action of $\mathbb{N}_{\geq 1}$ on the monotheta environment may be understood, for instance, by observing that the cyclotomic rigidity isomorphism

$$
\left(l \cdot \underline{\Delta}_{\Theta}\right) \otimes(\mathbb{Z} / N \mathbb{Z}) \stackrel{\sim}{\rightarrow} \Delta^{\left[\boldsymbol{\mu}_{N}\right]}
$$

[cf. Corollary 2.19, (i); Remark 2.19.3] is not compatible with the endomorphism of the "distinct cyclotome" $\Delta^{\left[\mu_{N}\right]}$ given by multiplication by $M \in \mathbb{N}_{\geq 1}$, unless $M \equiv 1$ modulo $N$. [Here, it is useful to recall that there is no natural action of $\mathbb{N}_{\geq 1}$ on $\underline{\Pi}_{\underline{\underline{X}}}^{\mathrm{tp}}$ that induces the multiplication action on $\mathbb{N}_{\geq 1}$ on $l \cdot \underline{\Delta}_{\Theta}$ !] Alternatively, this property may be regarded as a restatement of the interpretation [cf. Remark 2.19.3] of the cyclotomic rigidity of a mono-theta environment as a sort of "integral structure", or "basepoint", relative to the action of $\mathbb{N}_{\geq 1}$. Moreover, we remark that it is precisely the presence of this rigidity property that motivates the interpretation, stated above, of the "distinct cyclotome" as a sort of "Frobenius germ" - i.e., something that is somewhat less than the "full Frobenius structure" present in a Frobenioid, but which nevertheless serves as a sort of "trace", or "partial, but essential record", of such a "full Frobenius structure".

Remark 5.10.4

(i) Relative to the theory of $\S 2$, which does not involve Frobenioids, the significance of the introduction of Frobenioids may be understood as follows:

The use of Frobenioids allows one to consider, in a natural way, the monoid

$$
\left(\ddot{\Theta}(\sqrt{-1})^{-1} \cdot \ddot{\Theta}\right)^{\mathbb{N}}
$$

[cf. Theorem 5.7] of powers of the theta function. 
This is not possible for "abstract mono-theta environments", for numerous reasons, centering around the preservation of cyclotomic rigidity [cf. Remarks $2.19 .2,2.19 .3,5.10 .3$, as well as Remark 5.12.5 below]. One way to understand this phenomenon is to think of the various discrete monoids [i.e., "copies of $\left.\mathbb{N}^{\prime \prime}\right]$ that appear in the structure of a Frobenioid as imparting a rigidity to Frobenioids that takes the place of the cyclotomic rigidity of an abstract monotheta environment [cf. Remark 2.19.4]. From this point of view, the tempered Frobenioid $\mathcal{C}$ involved may be thought of as furnishing a sort of "discrete Tatemodule-like unraveling" — i.e., a means of passing from " $\mathbb{Q} / \mathbb{Z}(1)$ " to "No " as opposed to $\operatorname{Hom}(\mathbb{Q} / \mathbb{Z}(1), \mathbb{Q} / \mathbb{Z}(1))=\widehat{\mathbb{Z}}]$ — of the various abstract mono-theta environments that appear in the theory [cf. the notion of "Frobenius germs" discussed in Remark 5.10.3].

(ii) If one is only interested in the monoid " $\left(\ddot{\Theta}(\sqrt{-1})^{-1} \cdot \ddot{\Theta}\right)^{\mathbb{N}}$ " of (i), then it might appear at first glance that instead of working with the tempered Frobenioid $\mathcal{C}$, it suffices instead to work with the associated birationalization $\mathcal{C}^{\text {birat }}$. On the other hand, it is precisely by working with $\mathcal{C}$ [instead of $\mathcal{C}^{\text {birat }}$, i.e., by working with ample line bundles, that one may access the "numerator" and "denominator" of a meromorphic function as separate, independent entities. Moreover, it is this aspect of $\mathcal{C}$ that is of crucial importance in relating the Frobenioid-theoretic aspects of (i) to an abstract mono-theta [i.e., as opposed to bi-theta, which would force one to sacrifice discrete rigidity — cf. Remark 2.16.1] environment.

(iii) In addition to the significance of the use of Frobenioids as discussed in (i), more generally the introduction of Frobenioids is useful in that, unlike tempered fundamental groups, Frobenioids tend to be compatible with "Frobeniuslike" structures [e.g., structures that involve some sort of linear ordering] cf. the discussion of [Mzk17], Remark 3.1.3. By contrast, by thinking of an abstract mono-theta environment as arising from the tempered fundamental group [cf. Corollary 2.18], one may work with such mono-theta environments in situations in which it is important to be free of the constraints arising from such "Frobenius-like", "order-conscious" structures.

Before proceeding, we review a "well-known" result in category theory.

Lemma 5.11 (Non-category-theoreticity of Particular Morphisms in an Abstract Equivalence Class). Let $\mathcal{E}$ be a category; $G, H, I \in \mathrm{Ob}(\mathcal{E})$ distinct objects of $\mathcal{E} ; f: G \rightarrow H, g: G \rightarrow G$ morphisms of $\mathcal{E} ; \alpha_{G} \in \operatorname{Aut}_{\mathcal{E}}(G)$, $\alpha_{H} \in \operatorname{Aut}_{\mathcal{E}}(H), \alpha_{I} \in \operatorname{Aut}_{\mathcal{E}}(I)$ automorphisms. Then there exists a selfequivalence $\Xi: \mathcal{E} \stackrel{\sim}{\rightarrow} \mathcal{E}$ that induces the identity on $\mathrm{Ob}(\mathcal{E})$ and is isomor- 
phic to the identity self-equivalence via an isomorphism that maps

$$
\begin{aligned}
G \mapsto \alpha_{G} \in \operatorname{Aut}_{\mathcal{E}}(G) ; \quad H \mapsto \alpha_{H} \in \operatorname{Aut}_{\mathcal{E}}(H) ; \quad I \mapsto \alpha_{I} \in \operatorname{Aut}_{\mathcal{E}}(I) ; \\
J \mapsto \operatorname{id}_{J} \in \operatorname{Aut}_{\mathcal{E}}(J)
\end{aligned}
$$

- where $\mathrm{id}_{J}$ denotes the identity morphism $J \rightarrow J-$ for all $J \in \mathrm{Ob}(\mathcal{E})$ such that $J \neq G, H, I$. In particular, $\Xi$ maps $f \mapsto \alpha_{H} \circ f \circ \alpha_{G}^{-1}, g \mapsto \alpha_{G} \circ g \circ \alpha_{G}^{-1}$.

Proof. First, let us observe that by composing the self-equivalences obtained by applying Lemma 5.11 with two of the three automorphisms $\alpha_{G}, \alpha_{H}$, $\alpha_{I}$ taken to be the identity, we may assume without loss of generality that $\alpha_{H}$, $\alpha_{I}$ are the respective identity automorphisms of $H, I$. Now [one verifies immediately that] one may define an equivalence of categories [that is isomorphic to the identity self-equivalence]

$$
\Xi: \mathcal{E} \stackrel{\sim}{\longrightarrow} \mathcal{E}
$$

which restricts to the identity on $\mathrm{Ob}(\mathcal{E})$ and maps

$$
j \mapsto j \circ\left(\alpha_{G}\right)^{-1} ; \quad j^{\prime} \mapsto \alpha_{G} \circ j^{\prime} ; \quad j^{\prime \prime} \mapsto \alpha_{G} \circ j^{\prime \prime} \circ \alpha_{G}^{-1} ; \quad j^{\prime \prime \prime} \mapsto j^{\prime \prime \prime}
$$

for $j \in \operatorname{Hom}_{\mathcal{E}}(G, J), j^{\prime} \in \operatorname{Hom}_{\mathcal{E}}(J, G), j^{\prime \prime} \in \operatorname{Hom}_{\mathcal{E}}(G, G), j^{\prime \prime \prime} \in \operatorname{Hom}_{\mathcal{E}}\left(J, J^{\prime}\right)$, where $G \neq J, J^{\prime} \in \mathrm{Ob}(\mathcal{E})$. Thus, $\Xi$ satisfies the properties asserted in the statement of Lemma 5.11.

Finally, we apply the "general nonsense category theory" of Lemma 5.11 to explain certain aspects of the motivation that underlies the theory of monotheta environments.

Corollary $\mathbf{5 . 1 2}$ (Constant Multiple Indeterminacy of Systems). In the notation of Theorem 5.10, assume further that $N^{\prime} \geq 1$ is an integer such that $N$ divides $N^{\prime}$, but $M \stackrel{\text { def }}{=} N^{\prime} / N \neq 1 ; s_{N^{\prime}}^{\sqcap}, s_{N^{\prime}}^{\sqcup}: \bar{A}_{N^{\prime}} \rightarrow B_{N^{\prime}}$ an $N^{\prime}$-th root of a right fraction-pair of an l-th root of the theta function $\ddot{\Theta}$ such that there exists a pair of commutative diagrams

$$
\begin{array}{cc}
A_{N^{\prime}} \stackrel{s_{N^{\prime}}^{\sqcap}}{\longrightarrow} B_{N^{\prime}} \\
\downarrow \alpha_{N, N^{\prime}} & \downarrow^{\beta_{N, N^{\prime}}} \\
A_{N} \stackrel{s_{N}^{\square}}{\longrightarrow} & B_{N}
\end{array}
$$

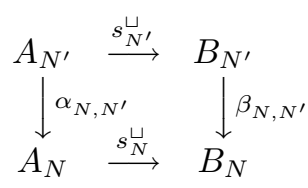

- where $\alpha_{N, N^{\prime}}$ (respectively, $\beta_{N, N^{\prime}}$ ) is an isometry of Frobenius degree $M \neq 1 ; \alpha_{N, N^{\prime}}$ is of base-Frobenius type [cf. Remark 4.3.2]. Then:

(i) The isomorphism classes of $A_{N}, B_{N}$, and $B_{N^{\prime}}$ are distinct. 
(ii) There exists a linear morphism $\iota: B_{N^{\prime}} \rightarrow B_{N}[c f$. the proof of Proposition 5.5].

(iii) Let

$$
\zeta: B_{N^{\prime}} \rightarrow B_{N}
$$

be either $\beta_{N, N^{\prime}}$ or $\iota$. Write $\left.\left(\mathcal{O}_{K}^{\times}\right)^{1 / N}\right|_{A} \subseteq \mathcal{O}^{\times}\left(A_{N}\right)$ for the subgroup induced by $\left(\mathcal{O}_{K}^{\times}\right)^{1 / N} \subseteq \mathcal{O}^{\times}\left(B_{N}\right)\left[c f\right.$. Lemma 5.8] via $s_{N}^{\square}$ or $s_{N}^{\sqcup} ;\left(\mathcal{O}_{K}^{\times}\right)^{*} \subseteq \mathcal{O}^{\times}\left(B_{N^{\prime}}\right)$ for the subgroup

$$
\left(\left(\mathcal{O}_{K}^{\times}\right)^{1 / N^{\prime}}\right)^{M / \operatorname{deg}_{\mathrm{Fr}}(\zeta)}
$$

[cf. Lemma 5.8]. [Thus, we have a natural $\boldsymbol{\mu}_{N^{\prime}}$-outer action of $\left(\mathcal{O}_{K}^{\times}\right)^{*} / \boldsymbol{\mu}_{N^{\prime}}\left(B_{N^{\prime}}\right)$ on $\operatorname{Aut}_{\mathcal{C}}\left(B_{N^{\prime}}\right)$ that is compatible, relative to $\zeta$, with the natural $\boldsymbol{\mu}_{N^{-o u t e r}}$ action of $\left(\mathcal{O}_{K}^{\times}\right)^{1 / N} / \boldsymbol{\mu}_{N}\left(B_{N}\right)$ on $\operatorname{Aut}_{\mathcal{C}}\left(B_{N}\right)$.] Then for any $\left.\kappa_{A} \in\left(\mathcal{O}_{K}^{\times}\right)^{1 / N}\right|_{A}$, $\kappa_{B} \in\left(\mathcal{O}_{K}^{\times}\right)^{1 / N}, \kappa^{\prime} \in\left(\mathcal{O}_{K}^{\times}\right)^{*}$, there exists a self-equivalence

$$
\Xi: \mathcal{C} \stackrel{\sim}{\longrightarrow} \mathcal{C}
$$

that is isomorphic to the identity self-equivalence via an isomorphism that maps

$$
\begin{gathered}
A_{N} \mapsto \kappa_{A}^{-1} \in \operatorname{Aut}_{\mathcal{C}}\left(A_{N}\right) ; \quad B_{N} \mapsto \kappa_{B}^{-1} \in \operatorname{Aut}_{\mathcal{C}}\left(B_{N}\right) ; \\
B_{N^{\prime}} \mapsto\left(\kappa^{\prime}\right)^{-1} \in \operatorname{Aut}_{\mathcal{C}}\left(B_{N^{\prime}}\right)
\end{gathered}
$$

and all other objects of $\mathcal{C}$ to the corresponding identity automorphism. In particular, $\Xi$ maps

$$
s_{N}^{\sqcap} \mapsto \kappa_{B}^{-1} \circ s_{N}^{\sqcap} \circ \kappa_{A} ; \quad s_{N}^{\sqcup} \mapsto \kappa_{B}^{-1} \circ s_{N}^{\sqcup} \circ \kappa_{A} ; \quad \zeta \mapsto \kappa_{B}^{-1} \circ \zeta \circ \kappa^{\prime} ;
$$

and

$$
\begin{aligned}
\operatorname{Im}\left(s_{N}^{\mathrm{trv}}\right)\left(\subseteq \operatorname{Aut}_{\mathcal{C}}\left(A_{N}\right)\right) & \mapsto \kappa_{A}^{-1} \cdot \operatorname{Im}\left(s_{N}^{\mathrm{trv}}\right) \cdot \kappa_{A} \subseteq \operatorname{Aut}_{\mathcal{C}}\left(A_{N}\right) ; \\
\operatorname{Im}\left(s_{N}^{\sqcap-\mathrm{gp}}\right)\left(\subseteq \operatorname{Aut}_{\mathcal{C}}\left(B_{N}\right)\right) & \mapsto \kappa_{B}^{-1} \cdot \operatorname{Im}\left(s_{N}^{\sqcap-\mathrm{gp}}\right) \cdot \kappa_{B} \subseteq \operatorname{Aut}_{\mathcal{C}}\left(B_{N}\right) ; \\
\operatorname{Im}\left(s_{N}^{\sqcup-\mathrm{gp}}\right)\left(\subseteq \operatorname{Aut}_{\mathcal{C}}\left(B_{N}\right)\right) & \mapsto \kappa_{B}^{-1} \cdot \operatorname{Im}\left(s_{N}^{\sqcup-\mathrm{gp}}\right) \cdot \kappa_{B} \subseteq \operatorname{Aut}_{\mathcal{C}}\left(B_{N}\right)
\end{aligned}
$$

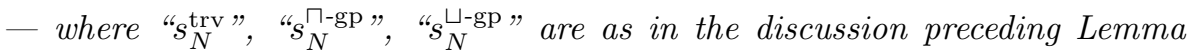
5.8 .

Proof. First, we consider assertions (i), (ii). Since $\beta_{N, N^{\prime}}$ is an isometry of Frobenius degree $M$, it follows that the pull-back via the morphism $B_{N^{\prime}}^{\text {bs }} \rightarrow$ $B_{N}^{\mathrm{bs}}$ of $\mathcal{D}$ of the line bundle that determines the object $B_{N}$ is isomorphic to the $M$-th tensor power of the line bundle that determines the object $B_{N^{\prime}}$. Moreover, it follows immediately from the discussion of line bundles in $\S 1$ [cf. 
the discussion preceding Proposition 1.1] that all positive tensor powers of these line bundles are nontrivial, and that the isomorphism classes of these line bundles are preserved by arbitrary automorphisms of $B_{N^{\prime}}^{\text {bs }}$. In particular, we conclude immediately that $B_{N}, B_{N^{\prime}}$ are non-isomorphic both to one another and to the Frobenius-trivial object [i.e., object defined by a trivial line bundle] $A_{N}$. Moreover, by multiplying by the $(M-1)$-th tensor power of the section of line bundles that determines either of the morphisms $s_{N^{\prime}}^{\square}, s_{N^{\prime}}^{\sqcup}: A_{N^{\prime}} \rightarrow B_{N^{\prime}}$ [cf. the discussion of Remark 5.12.5, (iii), below], we obtain a linear morphism $\iota: B_{N^{\prime}} \rightarrow B_{N}$. This completes the proof of assertions (i), (ii). Finally, in light of assertion (i), assertion (iii) follows immediately from Lemma 5.11.

Remark 5.12.1. Let $\mathcal{E}$ be a category. Then for any $E \in \mathrm{Ob}(\mathcal{E})$,

$$
\operatorname{Aut}_{\mathcal{E}}(E)
$$

has a natural group structure. Indeed, this group structure is precisely the group structure that allows one, for instance, to represent the group structure of a tempered topological group category-theoretically via temperoids or to represent [cf. Theorem 5.10, (iii)] the group structure portion of a mono-theta environment category-theoretically via tempered Frobenioids. On the other hand, in both of these cases, one is, in fact, not just interested in the group structure, but rather in the topological group structure of the various objects under consideration. From this point of view:

Temperoids [or, for that matter, Galois categories] allow one to represent the topological group structure under consideration via the use of numerous objects corresponding to the various open subgroups of the topological group under consideration, as opposed to the use of a "single universal covering object", whose automorphism group allows one to represent the entire group under consideration via a single object, but only at the expense of sacrificing the additional data that constitutes the topology of the topological group under consideration.

This approach of using "numerous objects corresponding to the various open subgroups" carries over, in effect, to the theory of tempered Frobenioids, since such tempered Frobenioids typically appear over base categories given by [the subcategory of connected objects of] a temperoid.

Remark 5.12.2. In general, if one tries to consider systems [e.g., projective systems, such as "universal coverings"] in the context of the "numerous objects 
approach" of Remark 5.12.1, then one must contend with the following problem: Suppose that to each object $E$ in some given collection $\mathcal{I}$ of isomorphism classes of objects of $\mathcal{E}$, one associates certain data

$$
E \mapsto \mathbb{D}_{E}
$$

[such as the group $\operatorname{Aut}_{\mathcal{E}}(E)$, or some category-theoretically determined subquotient of $\operatorname{Aut}_{\mathcal{E}}(E)$ ], which one may think of as a functor on the full subcategory of $\mathcal{E}$ consisting of objects whose isomorphism class belongs to $\mathcal{I}$. Then:

One must contend with the fact there is no natural, "categorytheoretic" choice [cf. Lemma 5.11] of a particular morphism $\zeta$ : $E \rightarrow F$, among the various composites

$$
\alpha_{F} \circ \zeta \circ \alpha_{E}
$$

- where $\alpha_{E} \in \operatorname{Aut}_{\mathcal{E}}(E), \alpha_{F} \in \operatorname{Aut}_{\mathcal{E}}(F)$ - for the task of relating $\mathbb{D}_{E}$ to $\mathbb{D}_{F}$.

In particular:

One necessary condition for the data constituted by the functor $E \mapsto$ $\mathbb{D}_{E}$ to form a coherent system is the condition that the data $\mathbb{D}_{E}$ be invariant with respect to the various automorphisms induced by the various $\operatorname{Aut}_{\mathcal{E}}(E)$ - i.e., that $\operatorname{Aut}_{\mathcal{E}}(E)$ act as the identity on $\mathbb{D}_{E}$.

One "classical" example of this phenomenon is the category-theoretic reconstruction of a tempered topological group from its associated temperoid [cf. [Mzk14], Proposition 3.2; the even more classical case of Galois categories], where one is obliged to work with topological groups up to inner automorphism [cf. also Remark 5.12 .8 below].

Remark 5.12.3. Now we return to our discussion of Frobenioid-theoretic mono-theta environments, in the context of Theorem 5.10, (iii). If, instead of working with "finite" mono-theta environments, one attempts to work with the projective system of mono-theta environments determined by letting $N$ vary [cf. Remark 2.16.1], then one must contend with the "constant multiple indeterminacy" of Corollary 5.12, (iii), relative to $\kappa^{\prime}$, of $\zeta=\beta_{N, N^{\prime}}$ [i.e., where we note that $\beta_{N, N^{\prime}}$ may be thought of as a typical morphism appearing in this projective system]. In particular, the existence of this indeterminacy implies that, in order to obtain the analogue of Theorem 5.10, (iii) - i.e., to describe, in a category-theoretic fashion, the relationship between a single abstract, static 
external projective system of mono-theta environments and the projective system of mono-theta environments constructed inside a tempered Frobenioid one must work with mono-theta environments up to the indeterminacies arising from the $\boldsymbol{\mu}_{N^{-}}$outer action of

$$
\operatorname{Aut}_{\mathcal{C}}\left(B_{N}\right) \supseteq \mathcal{O}^{\times}\left(B_{N}\right) \supseteq\left(\mathcal{O}_{K}^{\times}\right)^{1 / N} \rightarrow\left(\mathcal{O}_{K}^{\times}\right)^{1 / N} / \boldsymbol{\mu}_{N}\left(B_{N}\right) \stackrel{\sim}{\rightarrow} \mathcal{O}_{K}^{\times}
$$

[cf. Lemma 5.8]. That is to say, one must assume that one only knows the theta section portion of a mono-theta environment [cf. Definition 2.13, (ii), (c)] up to a constant multiple. Put another way, one is forced to sacrifice the constant multiple rigidity of Corollary 2.19, (iii). Moreover, we observe in passing that attempting to avoid sacrificing "constant multiple rigidity" by working with bi-theta environments [cf. the discussion preceding Lemma 5.8] does not serve to remedy this situation, since this forces one to sacrifice "discrete rigidity" [cf. Remark 2.16.1]. Thus, in summary:

A [finite, not profinite!] mono-theta enviroment serves in effect to maximize the rigidity, i.e., to minimize the indeterminacy, of the [l-th roots of the] theta function that one works with in the following three crucial respects:

(a) cyclotomic rigidity [cf. Corollary 2.19, (i); Remark 2.19.4];

(b) discrete rigidity [cf. Corollary 2.19, (ii); Remarks 2.16.1, 2.19.4];

(c) constant multiple rigidity [cf. Corollary 2.19, (iii); the discussion of the present Remark 5.12.3; Remark 5.12.5 below]

- all in a fashion that is compatible with the category-theoretic representation of the topology of the tempered fundamental group discussed in Remark 5.12.1.

This "extraordinary rigidity" of a mono-theta environment, along with the "bridging aspect" discussed in Remarks 2.18.2, 5.10.1, 5.10.2, 5.10.3, were, from the point of view of the author, the main motivating reasons for the introduction of the notion of a mono-theta environment.

Remark 5.12.4. With regard to the projective systems of mono-theta environments discussed in Remark 5.12.3, if, instead of trying to relate a single abstract such system to a Frobenioid-theoretic system, one instead takes the approach of relating, at each finite step [i.e., at each constituent object of the system], an abstract mono-theta environment to a Frobenioid-theoretic monotheta environment via Theorem 5.10, (iii) - hence, in particular, taking into 
account the "Aut ${ }_{\mathcal{C}}\left(B_{N}\right)$-orbit" indeterminacies that occur at each step when one considers such relationships - then one can indeed establish a categorytheoretic relationship between projective systems of mono-theta environments external and internal to the Frobenioid $\mathcal{C}$ without sacrificing the cyclotomic, discrete, or constant multiple rigidity properties discussed in Remark 5.12.3. Put another way, the difference between "attempting to relate whole systems at once as in Remark 5.12.3" and "applying Theorem 5.10, (iii), at each finite step" may be thought of as a matter of "how one arranges one's parentheses", that is to say, as the difference between

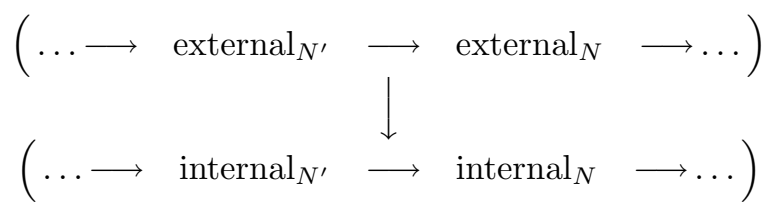

[i.e., the approach discussed in Remark 5.12.3, which forces one to sacrifice constant multiple rigidity] and

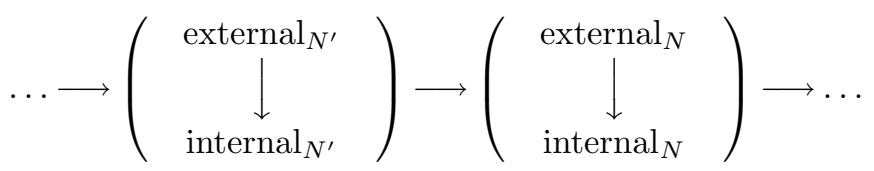

[i.e., "applying Theorem 5.10, (iii), at each finite step" — cf. the projective systems discussed in Corollary 2.19, (iii)].

Remark 5.12.5.

(i) Suppose that instead of working with first powers of $l$-th roots of the theta function, one tries instead to consider an $M$-th power version [where $M>1$ is an integer] of the mono-theta environment. As discussed in Remark 2.19.2, if one takes the most naive approach to doing this, then one must sacrifice cyclotomic rigidity in the sense that instead of obtaining cyclotomic rigidity for " $\boldsymbol{\mu}_{N}$ " [where, say, $N$ is divisible by $M$ ], one obtains cyclotomic rigidity only for the submodule " $M \cdot \boldsymbol{\mu}_{N} \subseteq \boldsymbol{\mu}_{N}$ ". Moreover, the resulting structures end up being intrisically indistinguishable [cf. Remark 2.19.3] from their first power counterparts, where " $N$ " is replaced by " $N / M$ ". In particular, in order to consider an " $M$-th power version" of the mono-theta environment in a meaningful fashion, one is led to consider, in addition, [the cyclotomic rigidity isomorphism arising from] some "first power version", together with data exhibiting the " $M$-th power version" as the $M$-th power of the "first power version". 
(ii) Thus, one is led, for instance, to consider systems consisting of the following data: (a) an $M$-th power version of the mono-theta environment; (b) a first power version [i.e., the usual version] of the mono-theta environment; (c) data relating these two versions [i.e., to the effect that the " $M$-th power version" is indeed obtained as the $M$-th power of the "first power version"]. Here, (c) amounts, in effect, to the consideration of morphisms such as the morphisms $\iota$ or $\beta_{N, N^{\prime}}$ of Corollary 5.12. In particular, the phenomenon discussed in Remark 5.12.2 manifests itself, i.e., one must contend with the constant multiple indeterminacy discussed in Corollary 5.12, (iii).

(iii) One approach to attempting to avoid the constant multiple indeterminacy that arose in (ii) [i.e., avoid the use of " $\iota$ ", " $\beta_{N, N}$, "] is to consider collections of data consisting of the following: (a) an $M$-th power version of the mono-theta environment; (b) the Frobenioid-theoretic cyclotomic rigidity isomorphism of Proposition 5.5. At first glance, this allows one to avoid the "constant multiple indeterminacy" that arises from working with "systems of objects" [cf. Remark 5.12.2], by restricting the data to data associated to a single object of the Frobenioid. Closer inspection, however, reveals a more complicated picture. Indeed, recall from the proof of Proposition 5.5 that the isomorphism of (b) was obtained by transporting, via linear morphisms, the cyclotomic rigidity isomorphism obtained from a first power version of the monotheta environment. Moreover, let us observe that this transportation operation is performed on the data $\operatorname{Aut}_{\mathcal{D}}\left((-)^{\mathrm{bs}}\right), \boldsymbol{\mu}_{N}(-)$ [or, more generally, $\mathcal{O}^{\times}(-)$] of, say, $B_{N^{\prime}}, B_{N}$ [cf. the proof of Proposition 5.5] - i.e., data which constitutes a sort of "semi-simplification" of the "extension structure" of a Frobenioid discussed in Remark 5.10.2. On the other hand, there is no evident way to extend such a transportation operation so as to apply to the "extension structure" [cf. Remark 5.10.2] of the exact sequence

$$
1 \rightarrow \mathcal{O}^{\times}(-) \rightarrow \operatorname{Aut}_{\mathcal{C}}((-)) \rightarrow \operatorname{Aut}_{\mathcal{D}}\left((-)^{\mathrm{bs}}\right) \rightarrow 1
$$

[for objects "(-)", such as $B_{N^{\prime}}, B_{N}$, which are Aut-ample - cf. the discussion preceding Lemma 5.8]. Put another way, to perform this transportation operation requires one to work up to an indeterminacy with respect to [so to speak "unipotent upper-triangular"] "shifting automorphisms" arising from cocycles of $\operatorname{Aut}_{\mathcal{D}}\left((-)^{\mathrm{bs}}\right)$ with coefficients in $\mathcal{O}^{\times}(-)\left(\supseteq \boldsymbol{\mu}_{N}(-)\right)$ [cf. the situation of Proposition 2.14, (ii)], which includes, in particular, a constant [i.e., $\mathcal{O}_{K^{-}}^{\times}$] multiple indeterminacy [cf. the collection of cocycles determined by the image of $\left(\mathcal{O}_{K}^{\times}\right)^{1 / N} / \boldsymbol{\mu}_{N}\left(B_{N}\right) \stackrel{\sim}{\rightarrow} \mathcal{O}_{K}^{\times}$in $H^{1}\left(\operatorname{Aut}_{\mathcal{D}}\left(B_{N}^{\text {bs }}\right), \boldsymbol{\mu}_{N}\left(B_{N}\right) !\right]$. More generally, by considering Kummer classes of log-meromorphic functions [such as the étale 
theta function! - cf. the proof of Corollary 5.12], it follows that this "shifting automorphism indeterminacy" implies, in particular, an indeterminacy which is, in effect, a sort of Galois/Kummer-theoretic translation of working with line bundles birationally. Note, moreover, that the cyclotomic rigidity isomorphism [cf. Corollary 2.19, (i)] arising from the specific splitting determined by the theta section is not preserved by [so to speak "unipotent upper-triangular"] "shifting automorphisms". Thus, in summary:

The operation of relating the isomorphism of (b) to the " $M$-th power version" of such a cyclotomic rigidity isomorphism arising from (a) i.e., exhibiting the " $M$-th power version" of (a) as the $M$-th power of the isomorphism of (b) - may only be performed after passing to the "semi-simplified data" $\operatorname{Aut}_{\mathcal{D}}\left((-)^{\mathrm{bs}}\right), \boldsymbol{\mu}_{N}(-)$.

On the other hand, applying the operation of "multiplication by $M$ " to the portion of the "semi-simplified data" constituted by $\boldsymbol{\mu}_{N}(-)$ amounts precisely to the situation discussed in Remark 2.19.3 [cf. also (i) above; Remark 5.10.3]. That is to say, this causes problems arising from the intrinsic indistinguishability of the domain and codomain copies of " $\boldsymbol{\mu}_{N}(-)$ " that occur when one multiplies by $M$. On the other hand, to attempt to assign "distinct labels" to these domain and codomain copies of " $\boldsymbol{\mu}_{N}(-)$ " amounts, in essence, to working with non-isomorphic tensor powers of some line bundle, i.e., to returning, in effect, to the situation discussed in (ii) above.

(iv) Finally, we note that another aspect of the lack of canonical splittings of the exact sequence $1 \rightarrow \mathcal{O}^{\times}(-) \rightarrow \operatorname{Aut}_{\mathcal{C}}((-)) \rightarrow \operatorname{Aut}_{\mathcal{D}}\left((-)^{\text {bs }}\right) \rightarrow 1$ of (iii) is that, relative to the automorphism indeterminacies discussed in Remark 5.12.2, there is no natural way to achieve a situation in which the inner automorphism indeterminacies of the tempered fundamental group [i.e., "Aut $\left((-)^{\mathrm{bs}}\right)^{\text {"] }}$ remain, but the constant multiple indeterminacies [i.e., " $\mathcal{O}^{\times}(-)$"] are eliminated.

Remark 5.12.6. At this point, it is useful to reflect [cf. Remark 2.19.4] on the significance of rigidifying the "constant multiple indeterminacy" and "shifting automorphism indeterminacy" of Remarks 5.12.3, 5.12.5. To this end, we observe that these types of indeterminacy are essentially multiplicative notions [cf. the cases discussed in Remark 2.19.4]. Thus, to work "modulo these sorts of indeterminacy" can only be done at the cost of sacrificing the additive structures [cf. Remark 2.19.4] implicit in the ring/scheme-theoretic origins of the various objects under consideration. Another important observation in this context is that the theory of Kummer classes depends essentially on the extension structure of a Frobenioid, i.e., breaks down completely if one "passes to 
semi-simplifications", as in Remark 5.12.5, (iii).

\section{Remark 5.12.7.}

(i) One way to understand the "constant multiple indeterminacy" phenomena observed in Remarks 5.12.3, 5.12.5 is as a manifestation of the nontriviality of the extension structure of a Frobenioid discussed in Remark 5.10.2.

(ii) Note that one way to attempt to avoid this "nontrivial extension structure of a Frobenioid" [which arises essentially from working with nontrivial line bundles] is to try to work strictly with rational functions [such as the theta function], i.e., to work with the birationalizations [i.e., in which "all line bundles becomes trivial"] of the Frobenioid "C" of Theorems 5.6, 5.10; Corollary 5.12. On the other hand, at the Galois/Kummer-theoretic level, this amounts to working strictly with étale theta functions [i.e., without theta environments], hence gives rise to the same "nondiscreteness phenomena" as those that appeared in the case of bi-theta environments [cf. Remarks 2.16.1; 5.10.4, (ii)].

Remark 5.12.8. Of course, the automorphism indeterminacy with regard to individual objects of a category discussed in Remark 5.12.2 also applies to the various base categories - i.e., [essentially] connected temperoids - of the [tempered] Frobenioids that appear in the above discussion. On the other hand, one verifies immediately that the various objects that we construct out of the associated tempered fundamental groups are invariant with respect to inner automorphisms in a fashion that is compatible with the topology of the tempered fundamental group [cf. Remark 5.12.1]. For instance, the construction of the tempered fundamental group of a connected temperoid involves projective systems that satisfy the analogue [cf. Remark 2.15.2] of the discrete rigidity property [cf. Corollary 2.19, (ii)] of a mono-theta environment. Moreover, the étale theta function is a collection of cohomology classes in the [continuous] cohomology of the tempered fundamental group, hence is invariant with respect to inner automorphisms.

Remark 5.12.9. Perhaps a sort of "unifying principle" underlying the "constant multiple indeterminacy" phenomena observed in Remarks 5.12.3, 5.12 .5 , on the one hand, and the discrete rigidity discussed in Remark 2.16.1, on the other, may be expressed in the following fashion:

Although at first glance, two [or many] pieces of data may appear to be likely to yield "more information" than "one" piece of data, in fact, the more pieces of data that one considers the greater the indeterminacies 
are that arise in describing the internal relations between these pieces of data.

Moreover, these greater indeterminacies may [as in the case of systems of objects of a Frobenioid in Remarks 5.12.3, 5.12.5, or bi-theta environments in Remark 2.16.1] ultimately result in "less information" than the information resulting from a single piece of data [cf. the discussion in the Introduction].

\section{Acknowledgements}

I would like to thank Akio Tamagawa, Makoto Matsumoto, Minhyong Kim, Seidai Yasuda, Kazuhiro Fujiwara, and Fumiharu Kato for many helpful comments concerning the material presented in this paper.

\section{References}

[André] Y. André, On a geometric description of $\operatorname{Gal}\left(\overline{\mathbb{Q}}_{p} / \mathbb{Q}_{p}\right)$ and a $p$-adic avatar of $\widehat{G T}$, Duke Math. J. 119 (2003), no. 1, 1-39.

[FC] G. Faltings and C.-L. Chai, Degeneration of abelian varieties, Springer, Berlin, 1990.

[LynSch] R. C. Lyndon and P. E. Schupp, Combinatorial group theory, Springer, Berlin, 1977.

[Mzk1] S. Mochizuki, Foundations of p-adic Teichmüller theory, Amer. Math. Soc., Providence, RI, 1999.

[Mzk2] - The absolute anabelian geometry of hyperbolic curves, in Galois theory and modular forms, 77-122, Kluwer Acad. Publ., Boston, MA, 2004.

[Mzk3] , The absolute anabelian geometry of canonical curves, Doc. Math. 2003, Extra Vol., 609-640 (electronic).

[Mzk4] , A survey of the Hodge-Arakelov theory of elliptic curves. I, in Arithmetic fundamental groups and noncommutative algebra (Berkeley, CA, 1999), 533-569, Proc. Sympos. Pure Math., 70, Amer. Math. Soc., Providence, RI, 2002.

[Mzk5] _ A survey of the Hodge-Arakelov theory of elliptic curves. II, in Algebraic geometry 2000, Azumino (Hotaka), 81-114, Adv. Stud. Pure Math., 36, Math. Soc. Japan, Tokyo, 2002.

[Mzk6] _. The Hodge-Arakelov theory of elliptic curves: Global discretization of local Hodge theories, RIMS Preprint Nos. 1255, 1256, 1999.

[Mzk7] _ The scheme-theoretic theta convolution, RIMS Preprint No. 1257, 1999.

[Mzk8] _ Connections and related integral structures on the universal extension of an elliptic curve, RIMS Preprint No. 1279, 2000.

[Mzk9] _. The Galois-theoretic Kodaira-Spencer morphism of an elliptic curve, RIMS Preprint No. 1287, 2000.

[Mzk10] The Hodge-Arakelov theory of elliptic curves in positive characteristic, RIMS Preprint No. 1298, 2000.

[Mzk11] _ The local pro- $p$ anabelian geometry of curves, Invent. Math. 138 (1999), no. $2,319-423$.

[Mzk12] _ The geometry of anabelioids, Publ. Res. Inst. Math. Sci. 40 (2004), no. 3, 819-881.

[Mzk13] (2005), 17-45 
[Mzk14] S. Mochizuki, Semi-graphs of anabelioids, Publ. Res. Inst. Math. Sci. 42 (2006), no. 1, 221-322.

[Mzk15] A combinatorial version of the Grothendieck conjecture, Tohoku Math. J. (2) 59 (2007), no. 3, 455-479.

[Mzk16] Absolute anabelian cuspidalizations of proper hyperbolic curves, J. Math. Kyoto Univ. 47 (2007), no. 3, 451-539.

[Mzk17] _ The geometry of Frobenioids I: The general theory, Kyushu J. Math. 62 (2008), 293-400.

[Mzk18] _ The geometry of Frobenioids II: Poly-Frobenioids, Kyushu J. Math. 62 (2008), 401-460.

[Mumf] D. Mumford, An analytic construction of degenerating abelian varieties over complete rings, Appendix to $[\mathrm{FC}]$. 